\title{
Institutionelle Mechanismen politischer Repräsentation in der Einwanderungsgesellschaft
}

Kumulative Dissertation zur Erlangung des Doktorgrades im Fach Politikwissenschaft (Dr. rer. pol.)

Eingereicht an der Fakultät Sozial-und Wirtschaftswissenschaften der Otto-FriedrichUniversität Bamberg

vorgelegt von

Lucas Geese, Dipl.-Pol. 


\section{Mitglieder der Promotionskommission}

Erstgutachter

Zweitgutachter

Weiteres Mitglied

Datum der Disputation: 23.07.2019

URN: urn:nbn:de:bvb:473-irb-470937

DOI: https://doi.org/10.20378/irb-47093
Prof. Dr. Thomas Saalfeld

Otto-Friedrich-Universität Bamberg

Prof. Dr. Marc Helbling

Otto-Friedrich-Universität Bamberg

Prof. Dr. Johannes Marx

Otto-Friedrich-Universität Bamberg 


\section{Inhaltsverzeichnis}

Rahmenpapier Institutionelle Mechanismen politischer Repräsentation in der Einwanderungsgesellschaft

Aufsatz 1 Geese, L. Do immigrant-origin candidates attract immigrant-origin voters in party-centred electoral systems? Evidence from Germany. Acta Polit (2018). https://doi.org/10.1057/s41269-018-0126-9

Aufsatz 2 Geese, L., \& Schacht, D. (2019). The more concentrated, the better represented? The geographical concentration of immigrants and their descriptive representation in the German mixed-member system. International Political Science Review, 40(5), 643-658. https://doi.org/10.1177/0192512118796263

Aufsatz 3 Lucas Geese \& Carsten Schwemmer (2019) MPs' principals and the substantive representation of disadvantaged immigrant groups, West European Politics, 42:4, 681-704, https://doi.org/10.1080/01402382.2018.1560196

Aufsatz 4 Lucas Geese (2019) Immigration-related Speechmaking in a Partyconstrained Parliament: Evidence from the 'Refugee Crisis' of the 18th German Bundestag (2013-2017), German Politics, https://doi.org/10.1080/09644008.2019.1566458 



\section{Rahmenpapier}

\section{Formale Kriterien}

Die vorliegende publikationsbasierte Dissertation erfüllt die Vorgaben und Regeln für kumulative Dissertationen der Fakultät für Sozial- und Wirtschaftswissenschaften der OttoFriedrich Universität Bamberg im Fach Politikwissenschaft, wie in folgenden Regelwerken definiert:

- Promotionsordnung der Fakultät Sozial- und Wirtschaftswissenschaften der OttoFriedrich-Universität Bamberg vom 31.03.2008

- Leitlinien für kumulative Dissertationen des Promotionsausschusses der Fakultät Sozial- und Wirtschaftswissenschaften, Stand Februar 2013

- Handreichung der Fachgruppe Politikwissenschaft zur Anwendung der Promotionsordnung bezüglich kumulativer Promotionen im Fach Politikwissenschaft in der Fassung vom 25.11.2015

Von diesen formuliert die Handreichung der Fachgruppe Politikwissenschaft die strengsten Kriterien. Daher werden diese als Referenz verwendet, um darzulegen, dass die vorliegende Dissertation die Vorgaben und Regeln für kumulative Dissertationen erfüllt.

Zu diesem Zweck gibt Tabelle 1 einen Überblick über die Aufsätze als Bestandteile der vorliegenden Dissertation, die 5-Jahres-Impact-Faktoren (laut ISI Web of Knowledge) der Zeitschriften, in denen die Aufsätze zur Veröffentlichung angenommen wurden, sowie die erreichten Punkte der Aufsätze nach dem Punktesystem der Handreichung.

Die vorliegende Dissertation besteht aus vier Aufsätzen und erfüllt damit die Vorgabe der Handreichung, dass eine kumulative Dissertation aus mindestens vier Aufsätzen bestehen muss. 


\section{Tabelle 1: Aufsatzübersicht}

\begin{tabular}{|c|c|c|c|}
\hline Aufsatz Nr. & Bibliographische Angabe & $\begin{array}{l}\text { 5-Jahres- } \\
\text { Impact-Faktor } \\
2017 \text { (laut ISI } \\
\text { Web of } \\
\text { Knowledge) }{ }^{1}\end{array}$ & $\begin{array}{l}\text { Punkte/ } \\
\text { Kategorie laut } \\
\text { Handreichung }\end{array}$ \\
\hline 1 & $\begin{array}{l}\text { Geese, Lucas, 2018, Do immigrant-origin } \\
\text { candidates attract immigrant-origin voters in } \\
\text { party-centred electoral systems? Evidence from } \\
\text { Germany, Acta Politica, } \\
\text { https://doi.org/10.1057/s41269-018-0126-9. } \\
\text { (ISSN: 0001-6810) }\end{array}$ & 1,373 & $3 / \mathrm{A}$ \\
\hline 2 & $\begin{array}{l}\text { Geese, Lucas/ Schacht, Diana, 2018, The more } \\
\text { concentrated, the better represented? The } \\
\text { geographical concentration of immigrants and } \\
\text { their descriptive representation in the German } \\
\text { mixed-member system, International Political } \\
\text { Science Review, } \\
\text { https://doi.org/10.1177/0192512118796263. } \\
\text { (ISSN: 0192-5121) }\end{array}$ & 1,891 & $3 / \mathrm{A}$ \\
\hline 3 & $\begin{array}{l}\text { Geese, Lucas/ Schwemmer, Carsten, 2019, MPs' } \\
\text { Principals and the Substantive Representation of } \\
\text { Disadvantaged Immigrant Groups, West European } \\
\text { Politics, } \\
\text { https://doi.org/10.1080/01402382.2018.1560196. } \\
\text { (ISSN: 0140-2382) }\end{array}$ & 3,100 & $4 / \mathrm{A}+$ \\
\hline 4 & $\begin{array}{l}\text { Geese, Lucas, 2019, Immigration-related } \\
\text { speechmaking in a party-constrained parliament: } \\
\text { evidence from the 'refugee crisis' of the 18th } \\
\text { German Bundestag (2013-2017), German Politics, } \\
\text { https://doi.org/10.1080/09644008.2019.1566458. } \\
\text { (ISSN: 0964-4008) }\end{array}$ & 1,312 & $3 / \mathrm{A}$ \\
\hline
\end{tabular}

\footnotetext{
${ }^{1}$ Informationen (Stand 17.01.2019) von https://jcr.incites.thomsonreuters.com/JCRLandingPageAction.action?wsid=E3VoHo9OWvl1zYmJXu5\&Init=Yes \&SrcApp=IC2LS\&SID=H5-tG3KWZqx2F6if7BWniSaY3Nfi8rvqDVhpp18x2dGm79kMaSj34VaOlgrsEC0Qx3Dx3DbTwhzrykzA7D3308Px2Fc6Fgx3Dx3DYwBaX6hN5JZpnPCj2IZNMAx3Dx3D-jywguyb6iMRLFJm7wHskHQx3Dx3D.
} 
Alle vier Aufsätze wurden in englischer Sprache in begutachteten (peer-reviewed) internationalen Fachzeitschriften zur Publikation akzeptiert. Damit werden die folgenden zwei Voraussetzungen erfüllt: Erstens wird die Bedingung der Handreichung erfüllt, dass mindestens zwei Aufsätze in Fachjournalen veröffentlicht sein müssen und zwei Aufsätze sich mindestens im Begutachtungsverfahren befinden müssen. Zweitens wird die Bedingung der Handreichung erfüllt, dass mindestens eine der Arbeiten in englischer Sprache zur Publikation in einer internationalen Fachzeitschrift mit Peer Review-Verfahren eingereicht ist.

Gemäß dem Punktesystem der Handreichung erreichen die vier Aufsätze dieser Dissertation 13 Punkte. Somit wird die Bedingung der Handreichung erfüllt, mindestens fünf Punkte insgesamt zu erreichen.

Sechs der 13 Punkte wurden in Allein-Autorenschaft erbracht, was die Bedingung der Handreichung erfüllt, mindestens drei Punkte in Allein-Autorenschaft zu erwerben.

Die Anteile der Autoren an den beiden Aufsätzen in Koautorenschaft gestalten sich wie folgt. Die originären Ideen des zweiten und dritten Aufsatzes sind die des Verfassers dieser Dissertation. Die Entwicklung der theoretischen Argumente sowie die Einbettung dieser in eine relevante Forschungsliteratur sind in beiden Aufsätzen hauptsächlich die Leistung des Verfassers. Die theoretischen Beiträge der Koautorin bzw. des Koautors haben sich demgegenüber hauptsächlich auf die gemeinsame Diskussion der Argumente beschränkt, welche vom Verfasser vorbereitet wurden. Im zweiten Aufsatz wurde die Datenerhebung und -vorbereitung überwiegend vom Verfasser geleistet, vor allem mit Blick auf die Erhebung und Kodierung der Kandidatendaten (unter Beihilfe studentischer Hilfskräfte), während sich der Beitrag der Koautorin im Wesentlichen auf die Erhebung von Strukturdaten der Wahlkreisebene erstreckt hat. Die empirische Analyse des Datenmaterials wurde im zweiten Aufsatz zu gleichen Teilen geleistet. Die Rohfassung des Fließtexts im zweiten Aufsatz wurde vom Verfasser geschrieben. Im Rahmen des Begutachtungsverfahrens wurde dieser dann jedoch immer wieder mit der Koautorin gemeinsam überarbeitet, wobei der Anteil des Verfassers insgesamt größer war (etwa 70:30). Im dritten Aufsatz war es die alleinige Leistung des Koautors, die analysierten Textkorpora zu sammeln. Die Entwicklung eines Kodierschemas wurde zu gleichen Teilen vom Verfasser und vom Koautor geleistet. Die Handkodierung mit Hilfe studentischer Hilfskräfte wurde vom Verfasser koordiniert, während die Implementierung des daran anschließenden automatisierten Kodierungsverfahrens vom Koautor geleistet wurde. Die statistische Datenanalyse wurde zu gleichen Teilen geleistet. Die Rohfassung des Fließtexts im dritten Aufsatz und die Überarbeitungen des Texts im Rahmen 
des Begutachtungsverfahrens wurden im Wesentlichen vom Verfasser geschrieben (etwa 95:5).

Kein Aufsatz wurde in Koautorenschaft mit einem Gutachter des Promotionsverfahrens erarbeitet. Dies erfüllt die Bedingung, dass höchstens bei einer Publikation ein Gutachter des Promotionsverfahrens beteiligt sein darf.

Darüber hinaus verlangt die Handreichung, dass die Schriften hinreichend unabhängig voneinander sind und zugleich ein schlüssiges Forschungsprogramm erkennen lassen, was durch eine den Veröffentlichungen vorangestellte inhaltliche Einordnung der Schriften zu demonstrieren ist. Auf mindestens 30 Seiten sind daher die zentralen Ergebnisse und originären Beiträge der Aufsätze zum Forschungsstand darzustellen. Diese Vorgaben werden mit dem folgenden Rahmenpapier erfüllt.

\section{Einleitung}

Das „Zeitalter der Migration“ (Castles et al., 2014) stellt westeuropäische Demokratien vor große Herausforderungen. Mehrere Zuwanderungswellen seit Ende des zweiten Weltkriegs haben die soziodemographische Zusammensetzung der Gesellschaften dieser Länder nachhaltig geprägt (Dancygier \& Laitin, 2014; Messina, 2007). Menschen mit Migrationshintergrund $^{2}$ stellen mittlerweile beachtliche zahlenmäßige Anteile sowohl an den Wohn- als auch Wahlbevölkerungen Westeuropas (Bird et al., 2011a). In Deutschland hat etwa jeder fünfte Einwohner und jeder zehnte Wähler ${ }^{3}$ einen Migrationshintergrund. Darüber hinaus hat die Migration innerhalb sowie nach Europa seither eher zu- denn abgenommen. Dies wurde vor allem im vergangenen Jahrzehnt offenbar, welches geprägt war von einer erhöhten EU-weiten Binnenmigration seit der Wirtschaftskrise von 2008 und einem verschärften Immigrationsdruck von Menschen, die vor Krieg und Armut aus Drittländern flüchteten (Moses, 2018). Themen der Immigration und der Integration von Einwanderern und ihren Nachkommen sind daher spätestens seit Anfang der 1980er Jahre wesentliche

\footnotetext{
${ }^{2}$ Im Rahmen dieser Dissertation wird der Begriff „Migrationshintergrund“ im Einklang mit der Definition des deutschen Statistischen Bundesamtes verwendet: „Eine Person hat einen Migrationshintergrund, wenn sie selbst oder mindestens ein Elternteil die deutsche Staatsangehörigkeit nicht durch Geburt besitzt"

(Statistisches Bundesamt, 2018, 4).

${ }^{3}$ Zur besseren Lesbarkeit werden in diesem Rahmenpapier personenbezogen Bezeichnungen, die sich zugleich auf Frauen und Männer beziehen, generell in der im Deutschen üblichen männlichen Form verwendet, also zum Beispiel „Wähler“ statt „WählerInnen“ oder „Wählerinnen und Wähler“. Die Verwendung der männlichen Form ist dabei stets geschlechtsunabhängig zu verstehen.
} 
Bestandteile der politischen Auseinandersetzung zwischen politischen Parteien, in den Medien und in der öffentlichen Meinung (Green-Pedersen \& Otjes, 2017; Helbling, 2014; Messina, 2007) und werden vermutlich noch lange den öffentlichen Diskurs und die politische Agenda maßgeblich beeinflussen.

Die vier Aufsätze der vorliegenden publikationsbasierten Dissertation beschäftigen sich vor diesem Hintergrund insbesondere mit Fragen der politischen Repräsentation. Politische Repräsentation ist das normative Kernanliegen des Demokratiemodells, das sich international durchgesetzt hat. Es ist ein zentrales Versprechen der modernen liberalen Demokratie, dass die Probleme aller Staatsbürger in einer fairen, d.h. nicht diskriminierenden Weise, im politischen Entscheidungsprozess repräsentiert werden (z.B. Dahl, 1971; Huber \& Powell, 1994; Mansbridge, 1999; Pitkin, 1967). Fritz Scharpf (1999, 16-20) hat dies als ,inputorientierte Legitimität" politischer Systeme bezeichnet.

Diesem Maßstab kommt eine besonders wichtige Bedeutung für jene Gesellschaftsteile zu, die sozial, ökonomisch und/oder politisch benachteiligt sind, was auch auf die Mehrheit der westeuropäischen Einwohner mit Migrationshintergrund zutrifft. In allen westeuropäischen Einwanderungsgesellschaften bestehen mehr oder weniger ausgeprägte „Schieflagen“. So sind Menschen mit Migrationshintergrund in diesen Ländern im Vergleich zur autochthonen Mehrheitsgesellschaft überproportional häufig von prekären Lebenslagen, geringeren Chancen der sozialen Mobilität sowie von alltäglichen Ressentiments und Diskriminierungen betroffen (Alba \& Foner, 2015; Dancygier \& Laitin, 2014; OECD \& EU, 2015). Die kulturelle, ökonomische und soziale Integration von Einwanderern und ihren Nachfahren, sowie die Gewährleistung von Chancengerechtigkeit wie dem Zugang zu wichtigen sozialen und ökonomischen Ressourcen, etwa im Bildungssystem oder auf dem Arbeitsmarkt, stellen daher wichtige Betätigungsfelder für Politikerinnen und Politiker in der Einwanderungsgesellschaft dar (siehe hierzu Alba \& Foner, 2015; Dancygier \& Laitin, 2014). Kurzum, politische Repräsentation ist zweifellos ein wichtiger Indikator der Benachteiligung und kann darüber hinaus einen wesentlichen Beitrag leisten, diese auf der Herkunft von Menschen beruhenden Disparitäten schrittweise zu reduzieren.

Unter migrationsorientierten Repräsentationsforschern ist es jedoch kein Geheimnis, dass die Idealvorstellung einer fairen Repräsentation mit Blick auf Bürger mit Migrationshintergrund von der Realität weit entfernt ist. In allen liberalen Demokratien westlicher Prägung bestehen mehr oder weniger ausgeprägte Defizite der politischen Repräsentation von Bürgern mit Migrationshintergrund (Alba \& Foner, 2009; Bird et al., 2011a; Bloemraad, 2013; Bloemraad 
\& Schönwälder, 2013; Dancygier, 2010). Vor dem Hintergrund dieser zwei Gemengelagen, das heißt einer weitgehenden gesellschaftlichen Benachteiligung einerseits und einem ausgeprägten Repräsentationsdefizit andererseits, ist die politikwissenschaftliche Erforschung der politischen Repräsentation dieser Gruppe(n) von besonderer gesellschaftlicher und demokratietheoretischer Relevanz. Daher kann es auch nicht überraschen, dass sich vor allem in den letzten zwei Dekaden eine wachsende Forschungsliteratur entwickelt hat, die die politische Repräsentation von Bürgern mit Migrationshintergrund in Westeuropa ins Zentrum des Interesses stellt (z.B. Bergh \& Bjorklund, 2011a; Bird et al., 2011b; Bloemraad \& Schönwälder, 2013; Dancygier, 2014; Dancygier et al., 2015; Hänni, 2017; Kittilson \& Tate, 2005; Kroeber, 2018; Messina, 2007; Ruedin, 2013, 2009; Saalfeld, 2011; Saalfeld \& Bischof, 2013; Sobolewska et al., 2018; Teney et al., 2010; Wüst, 2014a, 2016; Zingher \& Farrer, 2016). In einem Literaturüberblick von 2013 resümierten Karen Schönwälder und Irene Bloemraad allerdings noch, dass dieser Forschungsbereich sprichwörtlich noch in den Kinderschuhen stecke (Bloemraad \& Schönwälder, 2013, 572).

Migrations- und integrationspolitische Themen sind jedoch nicht nur für die politische Repräsentation von Bürgern mit Migrationshintergrund von Belang, sondern auch für die der autochthonen Mehrheitsbevölkerung. Dazu gehören Fragen, wie viele Arbeitsmigranten und/oder Asylbewerber aus welchen Ländern einwandern dürfen und inwieweit sie wohlfahrtstaatliche Leistungen und den Zugang zur Staatsbürgerschaft erhalten. Diese Themen können in der autochthonen Bevölkerung Ängste hinsichtlich eines Verlusts der eigenen nationalen und kulturellen Identität, der inneren Sicherheit und/oder der eigenen wirtschaftlichen Existenz schüren (Iversflaten, 2005; Rydgren, 2008; Scheve \& Slaughter, 2001). Das Erstarken radikaler rechtspopulistischer Parteien in Westeuropa kann daher auch als Gegenreaktion auf die Etablierung der Einwanderungsgesellschaft und einen anhaltenden Migrationsdruck interpretiert werden (Messina, 2007, Kapitel 3). Während migrationsrelevante politische Einstellungen und die Unterstützung radikaler rechtspopulistischer Parteien von Wählern relativ gut erforscht sind (siehe Goerres et al., 2018; Hainmueller \& Hopkins, 2014), ist weit weniger darüber bekannt, inwieweit die migrationspolitischen Wählerpräferenzen auch für das Handeln politischer Entscheidungsträger eine Rolle spielen (Hainmueller \& Hopkins, 2014, 227).

Die einzelnen Aufsätze der vorliegenden publikationsbasierten Dissertationsschrift sind somit einerseits als gesellschaftlich-problemorientierte Untersuchungen $\mathrm{zu}$ verstehen, andererseits aber auch als wichtige Beiträge zu einer sich entwickelnden empirischen und normativen 
politikwissenschaftlichen Forschungsliteratur. Im Sinne beider Aspekte verfolgen sie dabei das gemeinsame Ziel, unser Verständnis der Mechanismen politischer Repräsentation in der Einwanderungsgesellschaft zu verbessern. Diese Zielsetzung sowie ihre gesellschaftliche und wissenschaftliche Relevanz (vgl. King et al., 1994) stellen daher eines der vereinenden Elemente der insgesamt vier Beiträge dar. Zwei weitere Gesichtspunkte verbinden die vier Aufsätze darüber hinaus.

Zweitens stellen die Beiträge in ihrer Zusammenschau eine empirische Untersuchung parlamentarischer Repräsentation von Wählerinnen und Wählern bis hin zu Abgeordneten dar. Parlamente nehmen eine Schlüsselstellung in den Delegationsketten moderner repräsentativer Demokratien ein (Strøm, 2000). Als legislative Deliberations-, Kontroll- und Entscheidungsorgane sind sie nicht nur die demokratisch legitimiertesten Kollektivorgane im Sinne des Policy-Making, sondern nehmen auch die Aufgabe wahr, Bürger und Medien über politische Alternativen zu informieren (Geese et al., im Erscheinen.; Keh, 2015; Mansbridge, 1999). Dies macht Parlamente zu der zentralen Institution für politische Repräsentation in modernen liberalen Demokratien.

Das dritte einende Element besteht in einem gemeinsamen theoretischen Bezugsrahmen, der demokratische Repräsentationsprozesse als Prinzipal-Agent-Beziehungen auffasst und in der Tradition des Rational-Choice Institutionalismus das Verhalten der untersuchten Akteure (Wähler, Parteien und Parlamentarier) als Ergebnis des Zusammenwirkens zweier Variablengruppen versteht: zum einen werden die Ergebnisses kollektiven Handelns aus den Präfenzen der Akteure rekonstruiert; zum anderen werden sie auf der Grundlage dieser Präferenzen als strategisches Handeln innerhalb exogen gegebener institutioneller Anreizstrukturen modelliert (Diermeier \& Krehbiel, 2003; Gailmard, 2014; Hall \& Taylor, 1996). Das Erkenntnisinteresse aller vier Aufsätze bezieht sich in einem übergeordneten Sinn auf die Frage, wie demokratische Institutionen, das heißt genauer, wie Wahlsysteme, Regeln der parteiinternen Kandidatenaufstellung und parlamentsinterne Organisationsstrukturen den Repräsentationsprozess in der Einwanderungsgesellschaft strukturieren. Diesem übergeordneten Ziel intendieren die vier Aufsätze zu dienen, indem sie im Einzelnen folgende Forschungsfragen bearbeiten und dabei innovative Forschungsbeiträge leisten.

Aufsatz 1: Ist das Vorliegen eines kandidatenzentrierten Wahlsystems (z.B. Verhältniswahlsysteme mit offenen Listen oder Mehrheitswahlsystem in Einpersonenwahlkreisen) eine Bedingung dafür, dass Wähler mit Migrationshintergrund die Kandidatur von Personen mit Migrationshintergrund in das Kalkül ihrer Wahlentscheidung 
einbeziehen? Oder können dezentralisierte Kandidatennominierungsverfahren ähnliche Chancen für die Aufstellung und Wahl von Wahlbewerbern mit Migrationshintergrund generieren und dabei möglicherweise sogar gegenläufige Anreize eines parteizentrierten Wahlsystems (z.B. Verhältniswahlsystem mit geschlossenen Parteilisten) kompensieren? Diese mögliche Kompensation wird in der herkömmlichen Wahlsystemforschung zwar gelegentlich erwähnt (Norris, 2004, 232-233; Shugart et al., 2005, 441), ist aber empirisch noch nicht in ausreichender Breite erhärtet.

Aufsatz 2: Ist das Vorliegen eines Mehrheitswahlsystems in Einpersonenwahlkreisen eine wesentliche Bedingung für die geographische Repräsentation von Bürgern mit Migrationshintergrund durch Kandidaten mit Migrationshintergrund? Oder können dezentralisierte Kandidatenaufstellungsverfahren Anreize für Parteien erzeugen, auf geographische Konzentrationen von Wählern mit Migrationshintergrund mit einem geographisch maßgeschneiderten Angebot an entsprechenden Kandidaten zu reagieren? Und sofern der auf der Kandidatenaufstellung beruhende Kausalmechanismus existiert, funktioniert er selbst dann, wenn das Wahlsystem nach vorherrschender Lehrmeinung eigentlich geringe Anreize für geographisches Rekrutierungsverhalten generieren sollte (z.B. in Verhältniswahlsystemen mit geschlossenen Listen)?

Aufsatz 3: Wie beeinflussen komplexe Institutionenkombinationen von Kandidatennominierungsverfahren, Wahlsystemregeln und parlamentsinternen Organisationsstrukturen das Repräsentationsverhalten von Parlamentariern bezogen auf die gesellschaftliche Benachteiligung von Bürgern mit Migrationshintergrund? Trotz einer wachsenden Literatur bedürfen gesicherte Antworten auch auf diese Frage noch weitergehender Untersuchungen mit geeigneten Forschungsplänen.

Aufsatz 4: Ist eine höhere migrationspolitische Aufmerksamkeit des Redeverhaltens notwendigerweise das Resultat individueller Repräsentationsmotive? Oder strukturieren Parlamentsfraktionen als „kollektive Gatekeeper“ des Plenums Debatten unter bestimmten Bedingungen im Voraus, so dass die kollektiven Repräsentationsmotive der Parteien/Fraktionen die individuellen Motive von Parlamentariern insgesamt überlagern? Obwohl es in den letzten Jahren eine verstärkte theoretische und empirische Aufmerksamkeit hinsichtlich der Gatekeeper-Rolle von Fraktionen gab (z.B. Bäck \& Debus, 2016; Proksch \& Slapin, 2015), ist wenig darüber bekannt, wie die Repräsentation migrationsrelevanter Präferenzen in parlamentarischen Reden davon beeinflusst wird. 
Damit leisten die vier Beiträge in ihrer Zusammenschau auch einen übergeordneten repräsentationstheoretischen Beitrag, indem sie nicht nur zeigen wie institutionelle Erklärungsansätze etwa auf Fragen der Repräsentation benachteiligter sozialer Gruppen angewendet werden können, sondern auch empirische Erkenntnisse liefern, die unser Wissen insgesamt über die Konsequenzen dieser politischen Institutionen verbessern.

Darüber hinaus wahren alle Aufsätze ihre Eigenständigkeit in folgender Hinsicht: Erstens befassen sich jeweils zwei Aufsätze mit unterschiedlichen Dimensionen politischer Repräsentation, der deskriptiven und substantiellen Repräsentation (Mansbridge, 1999; Pitkin, 1967), die im folgenden Abschnitt genauer definiert werden. Zweitens unterscheiden sich die Aufsätze dabei in der Wahl der abhängigen Variablen, die sich jeweils auf das Verhalten unterschiedlicher Akteure des demokratischen Repräsentationsprozesses beziehen: das Wahlverhalten von Wählern mit Migrationshintergrund (Aufsatz 1), das Rekrutierungsverhalten politischer Parteien (Aufsatz 2), und das Verhalten von Parlamentariern in der Kammer (Aufsatz 3 und 4).

Um die Verknüpfung und Eigenständigkeit der einzelnen Beiträge besser zu veranschaulichen, präsentiert der folgende Abschnitt den übergeordneten gemeinsamen theoretischen Bezugsrahmen, der die Relevanz, Rollen und Interaktionen dieser drei Akteure im Zusammenhang mit politischer Repräsentation darlegt.

\section{2. Übergeordneter theoretischer Bezugsrahmen}

\subsection{Ein allgemeines Prinzipal-Agent-Modell demokratischer Repräsentation}

In seiner allgemeinsten Bedeutung kann der Begriff Repräsentation mit Pitkin definiert werden als: „the making present in some sense of something which is nevertheless not present literally or in fact“" (Pitkin, 1967, 9-10). Repräsentationsbeziehungen zwischen Bürgern und politischen Repräsentanten können somit als Prinzipal-Agent Beziehung verstanden werden, in der Prinzipale (Bürger) Agenten (Parteien und Parlamentarier) durch demokratische Wahl autorisieren, damit diese an ihrer Stelle politische Entscheidungen treffen (Lupia \& McCubbins, 2000; Mansbridge, 2003; Pitkin, 1967, 38-59; Strøm, 2000; Urbinati, 2011). Die 
Bürger verkörpern in diesem Modell daher das etwas ,which is not present literally or in fact“, während Repräsentanten die Aufgabe haben dieses etwas zu re-präsentieren.

Dieses in seiner Grundform leicht verständliche Modell, das bereits die wesentlichen Akteure (Wähler, Parteien und Parlamentarier) des parlamentarischen Repräsentationsprozesses identifiziert, stellt die Ausgangslage des übergeordneten theoretischen Bezugsrahmens dar, der die vier Aufsätze dieser Dissertation in einen Zusammenhang bringt.

Das analytische Erkenntnisinteresse von Prinzipal-Agent Modellen gilt vornehmlich der Auswahl und Verantwortlichkeit von Agenten gegenüber den Präferenzen ihrer Prinzipale in einer Repräsentationsbeziehung (z.B. Gailmard, 2014). In der politischen Repräsentationsforschung gilt das Erkenntnisinteresse in erster Linie der substantiellen Repräsentation der Präferenzen oder angenommenen Interessen ${ }^{4}$ der Bürger (Manin et al., 1999, 29). Pitkin definiert substantielle Repräsentation als: ,acting in the interest of the represented, in a manner responsive to them“ (Pitkin, 1967, 209). Die Präferenzen bzw. Interessen der Bürger fungieren in dem Konzept substantieller Repräsentation daher als Gradmesser der politischen Handlungsentscheidungen der Repräsentanten. Je stärker die Handlungsentscheidungen der Repräsentationsagenten auf der einen Seite mit den Interessen der Prinzipale auf der anderen Seite übereinstimmen, desto höhere Grade an substantieller Repräsentation werden erreicht.

Die substantielle Repräsentation des Prinzipals leidet allerdings dann, wenn Repräsentationsagenten eigene Ziele verfolgen, die nicht mit den Interessen der Prinzipale konform gehen. Solche Situationen erhöhen die Wahrscheinlichkeit, dass die Agenten sich auf Kosten des Prinzipals opportunistisch verhalten (sogenanntes „shirking“) (Lupia \&

\footnotetext{
${ }^{4}$ Streng genommen versteht der Prinzipal-Agent-Ansatz die subjektiven Präferenzen der Prinzipale und nicht ihre objektiven Interessen als maßgebend für das Handeln der Agenten. Objektive Interessen ergeben sich aus dem situativen Kontext der jeweils zu repräsentierenden Gruppe, also zum Beispiel auf Grundlage verfügbarer statistischer Indikatoren der sozialen Situation (Arbeitslosigkeit, Einkommen, Gesundheitsversorgung, usw.) (Swain, 1993, 6). Eine Verbesserung der sozialen Situation wäre dann im objektiven Interesse der Gruppe. Subjektive Interessen sind hingegen als konkrete politische Präferenzen definiert, also welche Politiken die Bürger selbst ganz konkret als ,in ihrem Interesse“ definieren (ebd., 6). Weil subjektive und objektive Interessen jedoch voneinander abweichen können, muss das Konzept der substantiellen Repräsentation laut Pitkin beide Arten von Interessen berücksichtigen: „[Substantive] representation does not require the principal to have formulated "a will" on issues before the representative, or even to know about them. One can represent others on matters they neither care nor know about. What the representative must do is act in his constituents' interests, but this implies that he must not normally come into conflict with their will when they have an express will. (...) He must not be found persistently at odds with the wishes of the represented without good reason in terms of their interest, without a good explanation of why their wishes are not in accord with their interest" (Pitkin, 1967, 162-163; ebd., 209-210). Zu analytischen Zwecken bezieht sich daher die Verwendung des Begriffs "Interessen" im weiteren Verlauf sowohl auf objektive Interessen als auch auf subjektive Präferenzen.
} 
McCubbins, 2000, 294). Um ein verantwortliches Verhalten der Agenten dennoch sicherzustellen, versucht der Prinzipal institutionelle Verhaltensanreize und Hemmnisse zu schaffen, damit Agenten auch bei abweichenden Präferenzen keine Entscheidungen treffen, die nicht im Interesse des Prinzipals sind (Gailmard, 2014). Dies lenkt den analytischen Blick auf die politischen Institutionen, die es dem Prinzipal erlauben, Informationsasymmetrien abzubauen, geeignete Agenten auszuwählen und das Verhalten von Agenten zu kontrollieren (Strøm, 2000).

In demokratischen Repräsentationssystemen ist diese Institution vor allem in dem Erfordernis von Wahlen verkörpert. Alle liberalen Demokratien zeichnen sich durch geheime, freie, gleiche und wiederkehrende Wahlen aus, welche somit im Zentrum des übergeordneten theoretischen Grundmodells stehen, das in Abbildung 1 dargestellt ist. Das Grundmodell identifiziert die drei für parlamentarische Repräsentation maßgeblichen Akteure: Wähler, Parteien und Parlamentarier. Wähler (als Prinzipale) stehen mit Parteien und Parlamentariern (als Repräsentationsagenten) in gegenseitigen Abhängigkeitsverhältnissen.

\section{Abbildung 1: Grundmodell politischer Repräsentation}

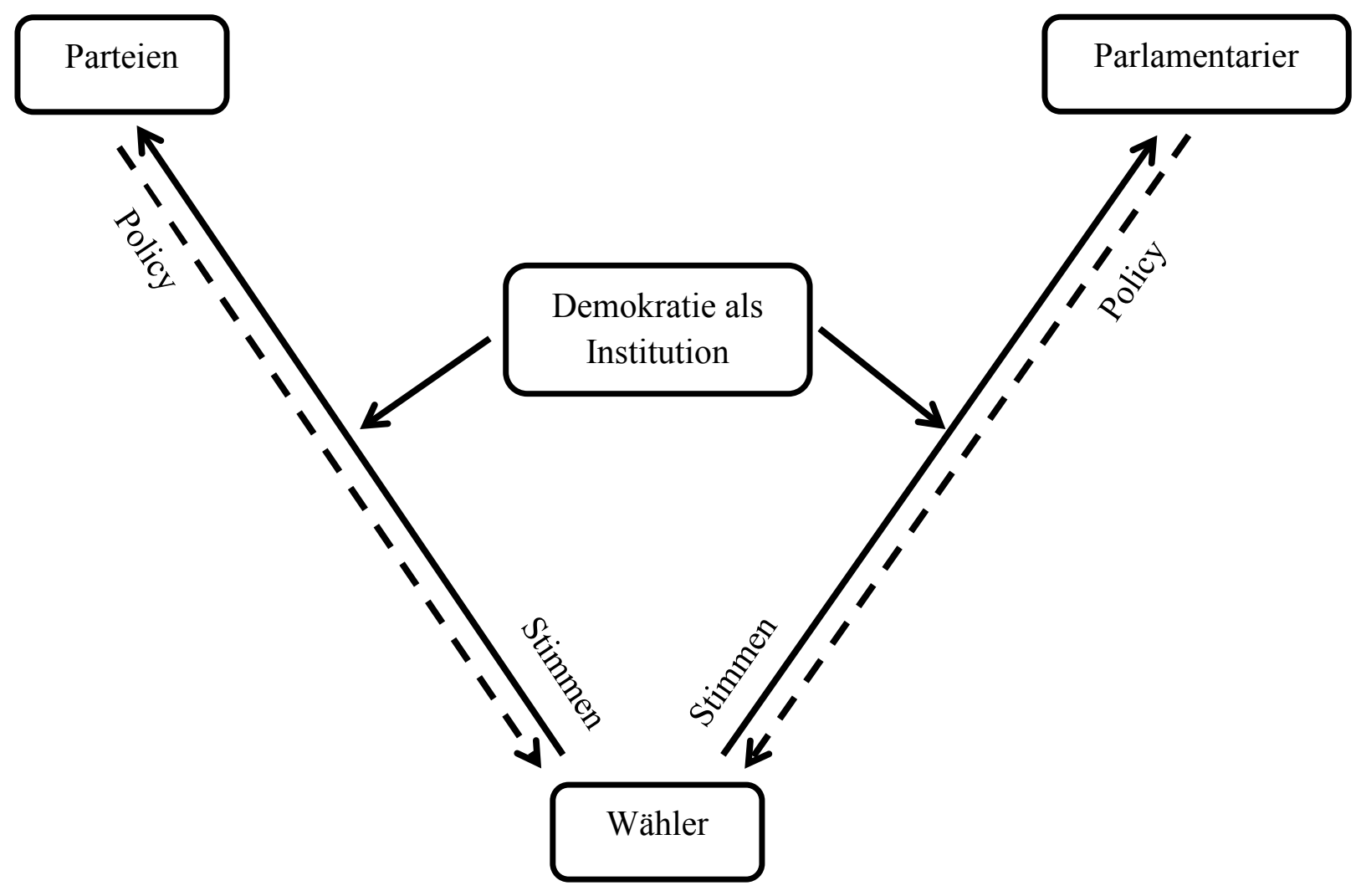


Prinzipal-Agent-Modelle sind in der Politikwissenschaft der Forschungstradition des Rational-Choice Institutionalismus zuzuordnen (Hall \& Taylor, 1996). Neben der Annahme exogen gegebener institutioneller Anreizstrukturen werden die Präferenzen oder Ziele der Akteure $\mathrm{zu}$ analytischen Zwecken als fixiert angenommen, so dass die Akteure unter ständiger Abwägung von Kosten-Nutzen Kalkülen strategische Handlungsentscheidungen treffen, um ihre aus methodologischen Gründen für den Untersuchungszeitraum als fixiert angenommenen Ziele zu erreichen (Diermeier \& Krehbiel, 2003; Hall \& Taylor, 1996; Shepsle, 2006). In dem in Abbildung 1 betrachteten Fall parlamentarischer Repräsentation, verfolgen Parteien und Parlamentarier das Ziel der Wiederwahl (Stimmenmaximierung; voteseeking) zum Zwecke des Machterhalts bzw. -zuwachses (Besetzung von Ämtern; officeseeking) und der Durchsetzung politischer Inhalte (policy-seeking) (Fenno, 1973; Mayhew, 1974; Strøm, 1997; Strøm \& Müller, 1999). Wähler verfolgen vor allem das Ziel, dass ihre Repräsentationsagenten Entscheidungen im Einklang mit den eigenen politischen Interessen treffen. Die Erreichung dieser Ziele hängt jedoch unter den Bedingungen der exogen gegebenen Institution „demokratische Wahlen“ vom Verhalten der jeweils anderen Akteure ab. Die Wiederwahl der Repräsentationsagenten hängt von den Wählern ab. Die Durchsetzung politischer Inhalte hängt von den Repräsentationsagenten ab. Die Akteure befinden sich daher in einer Art Tauschbeziehung. Jeder Akteur kontrolliert den Zugang zu einer Ressource, die der andere nutzen möchte.

Im Rahmen dieses theoretischen Grundmodells wird substantielle Repräsentation daher als ein durch demokratische Wahlen strukturiertes Phänomen betrachtet. Die Mechanismen werden dabei in der Möglichkeit der Wähler gesehen, ihre Repräsentationsagenten ex-ante und ex-post zu kontrollieren, das heißt bevor bzw. nachdem die Prinzipale die Agenten durch Wahlen autorisieren (Manin et al., 1999; Pitkin, 1967, 38-59; Strøm, 2000).

Die wesentliche ex-ante Kontrollmöglichkeit besteht im Screening des Universums potentieller Repräsentationsagenten mit anschließender Auswahl eines Mikrokosmos aus diesem Universum (Strøm, 2000, 271). Aus diesem Blickwinkel entsteht substantielle Repräsentation vor allem dadurch, dass Wähler Repräsentanten auswählen, die a priori bestimmte politische Inhalte umsetzen wollen, welche mit den Interessen der Wähler weitgehend konform gehen (Mansbridge, 2009, 370). Mansbridge implementiert diesen exante Repräsentationsmechanismus in dem Konzept der ,gyroscopic representation“:

„[V]oters affect political outcomes not by affecting the behaviour of the representatives [...], but by selecting and placing in the political system representatives whose 
behaviour is to some degree predictable based on their observable characteristics. (...) The representatives act like gyroscopes, rotating on their own axes [...] pursuing certain built-in [...] goals" (Mansbridge, 2003, 520-521).

Die ex-post Kontrollmöglichkeit besteht hingegen vor allem in dem wiederkehrenden Wahlerfordernis, das den Bürgern die Möglichkeit gibt, ihre Repräsentationsagenten in zukünftigen Wahlen zur Rechenschaft zu ziehen, das heißt durch Wiederwahl zu belohnen, oder durch Abwahl zu sanktionieren. Die Antizipation einer möglichen Abwahl in der Zukunft soll dabei die Repräsentanten veranlassen, von einem opportunistischen Verhalten (,shirking“) in der Gegenwart abzusehen (Fearon, 1999, 55). In diesem Sinne sind Mansbridges Konzepte der „promissory“ und „,anticipatory representation“ zu sehen. Das Konzept der ,promissory representation“ basiert auf der Idee, dass Repräsentanten vor der Wahl Versprechen gegenüber Wählern machen, die sie dann einlösen oder nicht (Mansbridge, 2003, 515). „Anticipatory representation” konzeptualisiert Repräsentanten als Akteure, die ihr Verhalten an der Erwartung ausrichten, welches Repräsentationsverhalten ihre Wähler bei der nächsten Wahl gutheißen, und nicht daran, welche Wahlversprechen sie vor der letzten Wahl gegeben haben (ebd., 515).

\subsection{Ein spezifisches Prinzipal-Agent-Modell politischer Repräsentation in der Einwanderungsgesellschaft}

Dieser allgemein gehaltene analytische Rahmen parlamentarischer Repräsentation kann erweitert und verfeinert werden. Abbildung 2 präsentiert einen angepassten Analyserahmen, der die Beiträge dieser Dissertation in einen spezifischeren Zusammenhang bringt. Während die Repräsentationsagenten (Parteien und Parlamentarier) dieselben wie im Grundmodell bleiben, werden jetzt sowohl Wähler mit als auch ohne Migrationshintergrund in der Rolle des Prinzipals gesehen. Außerdem werden die exogen gegebenen Institutionen als strukturierende Einflussfaktoren der Beziehungen zwischen den Akteuren präziser als Regeln des Wahlsystems, der Kandidatenaufstellung, sowie als parlamentsinterne Organisationsstrukturen definiert.

Ein erstes spezifisches Erkenntnisinteresse dieser Dissertation geht dabei der Forschungsfrage nach, unter welchen Bedingungen und aufgrund welcher institutionellen Mechanismen individuelle Parlamentarier die spezifischen Interessen benachteiligter Bürger mit 
Migrationshintergrund (Aufsatz 3) sowie die migrations- und integrationsspezifischen Themenprioritäten des Elektorats insgesamt (Aufsatz 4) substantiell repräsentieren. Beide Aufsätze stellen dadurch das Verhalten individueller Parlamentarier als Repräsentationsagenten von Wählern mit und Wählern ohne Migrationshintergrund (als Prinzipale) in den Mittelpunkt des Forschungsinteresses. Der Logik von Prinzipal-AgentBeziehungen folgend, stehen diesen Wählergruppen sowohl ex-ante als auch ex-post Kontrollen zur Verfügung, um das Verhalten ihrer Repräsentationsagenten entsprechend zu beeinflussen.

Abbildung 2: Angepasstes Grundmodell für die Analyse politischer Repräsentation in der Einwanderungsgesellschaft

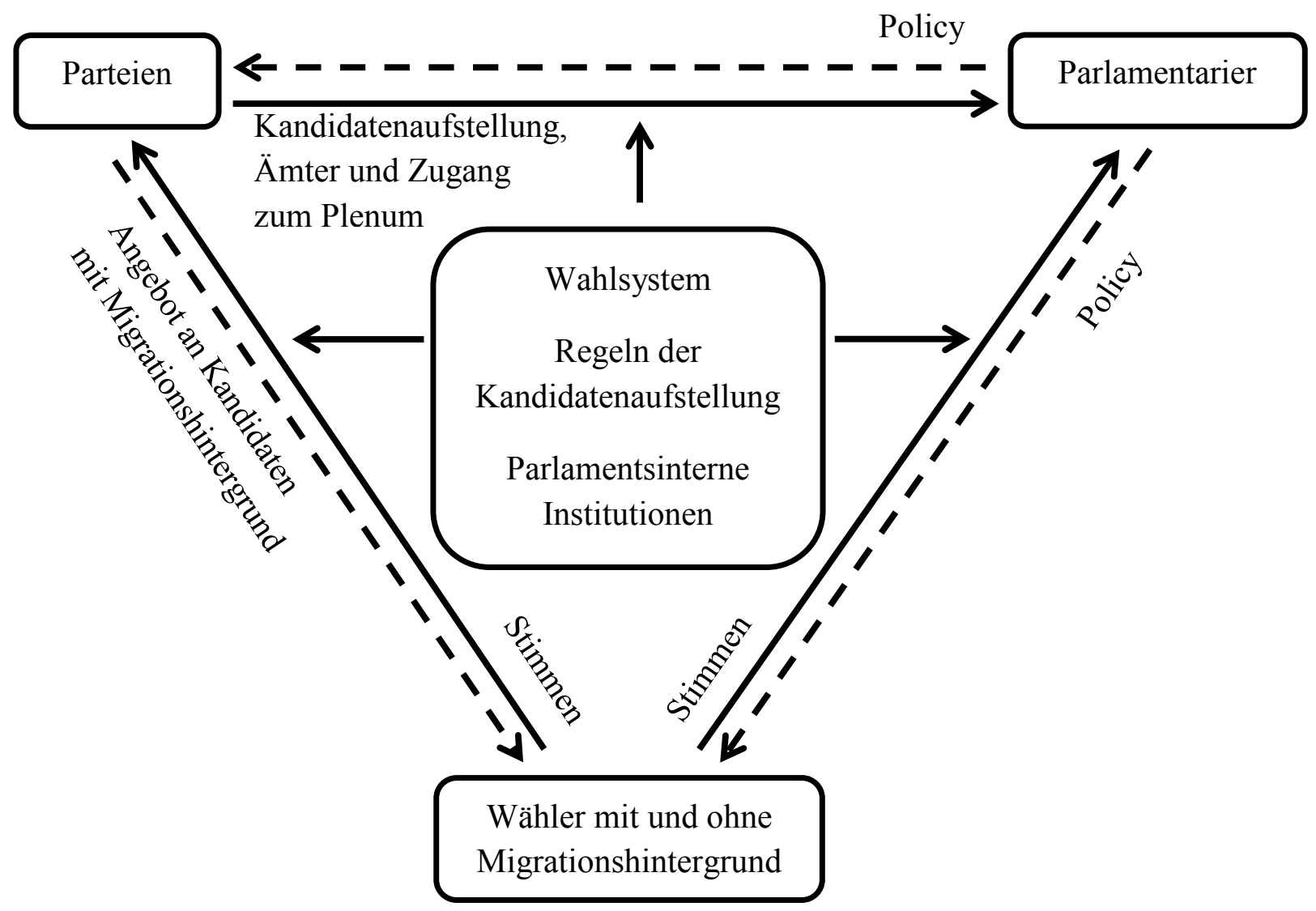

Eine analytische Perspektive, welche das Verhalten von Parlamentariern als ein durch die exante Kontrollmöglichkeiten der Wähler determiniertes Phänomen konzeptualisiert, rückt die individuellen Präferenzen von Parlamentariern als Resultat ihrer lebenslangen Erfahrungen in den Mittelpunkt (Bäck et al., 2014; Searing, 1991). Nach Mansbridges Konzept der „gyroscopic representation“ (2003) entsteht substantielle Repräsentation vor allem durch die 
Auswahl bestimmter Repräsentanten, deren politisches Problembewusstsein, inhaltliche Schwerpunktsetzung und Überzeugung den Interessen der Wähler möglichst nahe kommen. Um die Informationskosten bei der Einschätzung dieser Eigenschaften der potentiellen Repräsentanten gering zu halten, verlassen sich Wähler häufig auf sogenannte „candidate cues“. Damit sind leicht erfassbare Profilmerkmale der zur Wahl stehenden Kandidaten gemeint (z.B. soziodemographische Merkmale wie Namen, Herkunft, Geschlecht oder Merkmale der Parteizugehörigkeit), aus denen Wähler persönliche Erfahrungen sowie politische Positionen und Schwerpunktsetzungen der Kandidaten ableiten (Mansbridge, 2003, 521; McDermott, 1997, 1998; Shugart et al., 2005). Für soziodemografisch definierbare Gruppen, die sich in einer Position der gesellschaftlichen Benachteiligung befinden, sollte in diesem Zusammenhang vor allem deskriptive Repräsentation ${ }^{5}$ eine Rolle spielen, also die Präsenz von Repräsentanten, die der eigenen soziodemografisch definierten Gruppe zugehörig sind (Mansbridge, 1999; Phillips, 1995, 1998). Übertragen auf die substantielle Repräsentation von Bürgern mit Migrationshintergrund, lenkt dies den Blick vor allem auf die Präsenz von Parlamentariern mit Migrationshintergrund. Sofern das deskriptive Merkmal „Migrationshintergrund“ eine valides Indiz ist für spezifische Erfahrungen der Migration, gesellschaftlicher Benachteiligung und/oder Erfahrungen, die im Zuge der eigenen kulturellen und gesellschaftlichen Integration gemacht wurden, sollten diese Parlamentariertypen auch ein ausgeprägtes Problembewusstsein und intrinsisch motiviertes Interesse hinsichtlich der Probleme und Herausforderungen der Einwanderungsgesellschaft haben (siehe etwa Blätte \& Wüst, 2017; Wüst, 2014a).

Erklärungsansätze, die ex-post Kontrollmöglichkeiten von Wählern als Erklärung für legislatives Verhalten hervorheben, modellieren Parlamentarier hingegen als strategische Akteure, die eine Zieletrias aus Wiederwahl, Ämtern und Durchsetzung politischer Inhalte verfolgen (Carey, 2009; Mayhew, 1974; Strøm, 1997). Die Wiederwahl wird gemeinhin als wichtigstes Ziel angesehen, da die Erreichung der anderen politischen Ziele von diesem abhängen. Durch die Antizipation einer möglichen Abwahl sollten sich Parlamentarier dann im Sinne von Mansbridges ,anticipatory representation“ (2003) dazu veranlasst sehen, die Interessen ihrer Wähler zu repräsentieren (siehe auch Fearon, 1999, 55). Sofern Wähler mit Migrationshintergrund in den Augen von Parlamentariern also relevante Wählergruppen

\footnotetext{
${ }^{5}$ Deskriptive Repräsentation definiert das ,,something which is not present literally or in fact“ als soziodemographische Merkmale des Prinzipals, etwa ethnische, geschlechterspezifische oder soziale Klassenzugehörigkeit (siehe auch Mansbridge, 1999, 629). Frauen repräsentieren Frauen, Arbeiter repräsentieren Arbeiter und Menschen mit Migrationshintergrund repräsentieren Menschen mit Migrationshintergrund.
} 
darstellen, sollte für diese Parlamentarier auch ein elektoraler Anreiz bestehen, die Interessen dieser Gruppen zu repräsentieren. Dies gilt in ähnlicher Weise auch für Wählergruppen, die keinen Migrationshintergrund haben. Sofern diese sanktionsrelevante Gruppen sind, sollten Parlamentarier grundsätzlich auch elektorale Anreize haben, die migrations- und integrationsspezifischen Interessen dieser Gruppen zu vertreten.

Diese zwei analytischen Perspektiven vereint, dass sie die individuellen (intrinsischen oder strategischen) Repräsentationsmotive von Parlamentariern als Ursachen für substantielle Repräsentation sehen. Beide Betrachtungsweisen ignorieren jedoch, dass Parlamentarier gleichzeitig auch Bestandteil kollektiver Akteure sind, das heißt Mitglieder von Parteien innerhalb und außerhalb des Parlaments.

In dieser Hinsicht können Parlamentarier auch als Agenten von Parteiakteuren (als Prinzipale) verstanden werden (Carey, 2009; Müller, 2000; Proksch \& Slapin, 2015; Saalfeld \& Strøm, 2014). Diese Beziehung ist in Abbildung 2 als horizontale Wechselbeziehung zwischen Parlamentariern und Parteien dargestellt und trägt dem Umstand Rechnung, dass die kollektiven Stimmen-, Ämter- und inhaltlichen Ziele von Parteien (Strøm \& Müller, 1999) mit den individuellen Repräsentationsmotiven von Parlamentariern in Konflikt geraten können. Innerhalb des Parlaments sind hierarchisch organisierte Parteifraktionen eine notwendige Voraussetzung für eine effiziente Gestaltung des parlamentarischen Entscheidungsprozesses, etwa mit Blick auf Abstimmungen über die Sitzungsagenda oder Gesetzesvorlagen (Bowler et al., 1999; Cox, 2006; Saalfeld \& Strøm, 2014; Sieberer, 2006). Der Parteilinie widersprechendes Verhalten der Parlamentarier, welches zum Beispiel das Ziel hat eine persönliche Reputation gegenüber Wählern zu pflegen, kann jedoch gleichzeitig dem Ansehen und der Glaubwürdigkeit der Partei als einheitlicher Akteur schaden, und somit die kollektiven Stimmenmaximierungsziele der Partei gefährden (Carey \& Shugart, 1995; Snyder \& Ting, 2002).

Um eigene Parlamentarier „auf Linie zu halten“, können Parteieliten individuellen Parlamentariern jedoch (damit drohen,) wichtige Ressourcen vor(zu)enthalten, die für die Erreichung der individuellen Ziele der Parlamentarier instrumentell sind. Außerhalb des Parlaments kontrollieren Parteiorganisationen in fast allen westeuropäischen Demokratien als „Gatekeeper“ etwa die Auswahl von Parlamentskandidaten und können somit über die Wiederwahl einzelner Parlamentarier effektiv vorentscheiden (Hazan \& Rahat, 2010; Müller, 2000; Norris \& Lovenduski, 1995). Innerhalb des Parlaments kontrollieren die Fraktionsführungen als „Gatekeeper“ den Zugang zum Rednerpult in Parlamentssitzungen 
und somit eine Möglichkeit der öffentlichkeitswirksamen Kommunikation mit den Wählern (Bailer, 2017; Proksch \& Slapin, 2015). Wenn man Parlamentariern darüber hinaus nicht nur das Ziel der Wiederwahl sondern auch Ambitionen bezüglich einflussreicher und prestigeträchtiger Ämter innerhalb von Parlamentsfraktionen, Ausschüssen und Regierungen unterstellt (Strøm, 1997), dann sind Fraktionsführungen, die über die Besetzung dieser Ämter entscheiden, auch in dieser Hinsicht wichtige Prinzipale individueller Parlamentarier. Parteiakteure innerhalb und außerhalb des Parlaments müssen daher neben Wählern als weitere wichtige Prinzipale von Parlamentariern (als Repräsentationsagenten) betrachtet werden (Carey, 2009; Converse \& Pierce, 1986, 664-96).

Individuelle Parlamentarier haben somit einen Anreiz, unterschiedliche Erwartungen unterschiedlicher Prinzipale zu erfüllen, wodurch sie sich häufig in der schwierigen Situation befinden, gegensätzliche Erwartungen abwägen zu müssen. Welche Prinzipale diese Kalküle stärker dominieren hängt dabei vor allem davon $a b$, welcher Prinzipal die relativ folgenreicheren Belohnungs- und Sanktionsmittel in den Händen hält (Carey, 2009, 14) und den Zugang zu bestimmten Parlamentsaktivitäten kontrolliert (Keh, 2015; Proksch \& Slapin, 2015). Dies wird wesentlich durch die exogen gegebene institutionelle Umwelt der Parlamentarier beeinflusst. In dieser Hinsicht richten die beiden Aufsätze dieser Dissertation zur substantiellen Repräsentation ihr Augenmerk besonders auf die Ausgestaltung des Wahlsystems, der parteiinternen Kandidatenaufstellung und auf parlamentsinterne Organisationsstrukturen als institutionelle Anreiz- bzw. Hemmnisstrukturen. Nähere Ausführungen dazu folgen unter Abschnitt 4.

Ein zweites Erkenntnisinteresse bezieht sich im Gegensatz dazu auf die deskriptive Repräsentation von Bürgern mit Migrationshintergrund. Dies ist das Thema der ersten zwei Aufsätze dieser Dissertation, welche zu diesem Zweck die Repräsentationsbeziehung zwischen Wählern mit Migrationshintergrund und Parteien in den Vordergrund stellen (vgl. Abbildung 2). Wie bereits erwähnt, wird die substantielle Repräsentation von gesellschaftlich benachteiligten sozialen Gruppen durch eine adäquate deskriptive Repräsentation, das heißt durch die parlamentarische Präsenz von Gruppenmitgliedern, positiv beeinflusst ${ }^{6}$

\footnotetext{
${ }^{6}$ Laut Mansbridge (1999, 643-648) und Phillips (1998, 234-236) bestehe ohne deskriptive Repräsentation die Gefahr, dass die spezifischen Interessen gesellschaftlich benachteiligter Gruppen, vor allem in „,contexts of uncrystallized interests“, unzureichend repräsentiert werden. Damit sind Situationskontexte gemeint, in denen gruppenrelevante Themen zu plötzlich auf der parlamentarischen Agenda erscheinen, als dass Parteiprogramme und/oder Wählerpräferenzen bereits artikuliert wären, um Repräsentanten eindeutige Handlungsanleitungen zu geben. In diesen Situationen seien eigene Erfahrungen der Benachteiligung und
} 
(Mansbridge, 1999; Phillips, 1995, 1998). Dieser Zusammenhang wurde bereits vielfach empirisch bestätigt (z.B. Broockman, 2013; Hänni, 2017; Saalfeld, 2011; Saalfeld \& Bischof, 2013; Wüst, 2014a), so dass deskriptive Repräsentation als ein Indikator für substantielle Repräsentation verstanden werden kann. Damit stehen die ersten beiden Aufsätze dieser Dissertation auch in einem größeren Zusammenhang mit den anderen beiden Aufsätzen.

Parteien spielen in parlamentarischen Demokratien eine besondere Rolle für die soziodemografische Zusammensetzung von Parlamenten, weil in ihnen die Rekrutierung von Parlamentskandidaten stattfindet (Hazan \& Rahat, 2010; Müller, 2000; Norris \& Lovenduski, 1995). Als kollektive Akteure, die das Ziel verfolgen, Stimmen- und Sitzanteile in Parlamenten zu maximieren (Downs, 1957; Strøm \& Müller, 1999), sehen sich westeuropäische Parteien jedoch seit einigen Jahrzehnten abnehmenden Parteiloyalitäten (siehe etwa Dalton \& Wattenberg, 2000) ausgesetzt, von der vielfach angenommen wird, dass sie mit einer zunehmenden Individualisierung bzw. Personalisierung der politischen Repräsentationsbeziehung zwischen Wählern auf der einen und Parteien sowie Parlamentariern auf der anderen Seite einhergeht (Karvonen, 2010; Rahat \& Kenig, 2018; Renwick \& Pilet, 2016). In diesem Kontext kommt der Kandidatenauswahl eine größere Bedeutung für die Mobilisierung von Wählergruppen zu: „the nomination of more women or minority group members or more members of some religious sect or more workers or more farmers signals something about the party as a whole" (Katz, 2001, 278; siehe auch Mair et al., 2004). Die in den letzten Jahrzehnten gleichzeitig zunehmende ethnische Diversifizierung des „Wählermarktes“ aufgrund von Einwanderung lässt daher vermuten, dass die Rekrutierung von Kandidaten mit Migrationshintergrund eine Parteistrategie darstellt, die das Ziel hat, ein wachsendes Elektorat von Wählern mit Migrationshintergrund zur Stimmabgabe für die eigenen Partei zu mobilisieren (Bloemraad \& Schönwälder, 2013; Fonseca, 2011; Kittilson \& Tate, 2005; Laurence \& Maxwell, 2012).

Kandidaten mit soziodemographischen Merkmalen, die mit gesellschaftlichen Benachteiligungen und Diskriminierungen assoziiert sind, liefern Wählern bestimmte „cues“, durch die Kandidaten als vertrauenswürdiger und engagierter wahrgenommen werden, um die Interessen gesellschaftlich benachteiligter und diskriminierter Gruppen zu vertreten (Banducci et al., 2004; Landa et al., 1995; McDermott, 1998). Durch die Wahrnehmung einer Schicksalsgemeinschaft (,linked fate"), das heißt die Wahrnehmung, dass eine Verbesserung

Diskriminierung auf Seiten der deskriptiven Repräsentanten eine Voraussetzung, um gruppenrelevante Themen als solche auch erkennen zu können. 
der eigenen Situation von einer Verbesserung der Situation der Gruppe insgesamt abhängt (Dancygier \& Saunders, 2006; Dawson, 1994; Sanders et al., 2014), sollten Wähler mit Migrationshintergrund daher auf die Migrationsprofile der Kandidaten mit einem Wahlverhalten reagieren, das Parteien für entsprechende Kandidatenangebote belohnt (McConnaughy et al., 2010).

Die Komponenten der Tauschbeziehung zwischen den hier betrachteten Akteuren sind also die Stimmen der Wähler mit Migrationshintergrund und die angebotenen Kandidaten mit Migrationshintergrund auf Seiten der Parteien (vgl. Abbildung 2). Die ersten zwei Aufsätze dieser Dissertation beschäftigen sich daher mit dem Wahlverhalten von Wählern mit Migrationshintergrund (Aufsatz 1) und dem Rekrutierungsverhalten von Parteien (Aufsatz 2). Beide Aufsätze betrachten dabei jedoch die Verhaltensweisen der jeweiligen Akteure in Abhängigkeit von ihrer exogen gegebenen institutionellen Umwelt, definiert als Wahlsysteme sowie parteiinterne Regeln der Kandidatenaufstellung. Möglicherweise spielen Wahlsysteme ein kritische Rolle für die beschriebene Tauschbeziehung zwischen Wählern und Parteien, weil sie beeinflussen, inwieweit Wähler relativ stärker kandidatenorientiert oder parteiorientiert wählen (Carey \& Shugart, 1995) und inwieweit Parteien Anreize haben, eher territorial oder national definierte Wählergruppen in der Kandidatenauswahl zu berücksichtigen (Latner \& McGann, 2005). Auch Regeln und Praktiken der Kandidatenauswahl können in ähnlicher Weise entweder zentralisiert auf der gesamtstaatlichen Ebene oder aber territorial dezentralisiert auf der lokalen Ebene stattfinden, und somit stärkere Anreize für Parteien und Kandidaten generieren, entweder national oder lokal orientierte Mobilisierungsstrategien zu verfolgen (Hazan \& Rahat, 2010). Nähere Ausführungen zu diesen Zusammenhängen folgen in Abschnitt 3.

\subsection{Zur Rolle formaler theoretischer Modelle in der vergleichenden Politikwissenschaft}

Bevor die genaueren Kausalmechanismen innerhalb des beschriebenen theoretischen Grundmodells in den folgenden Abschnitten weiter spezifiziert werden, gilt es an dieser Stelle, den Generalisierungsanspruch der theoretischen Argumente zu klären sowie der Frage nachzugehen, in welchem Zusammenhang dieser mit der empirischen Analyse steht. Auch wenn alle Aufsätze empirisch am Fall des Deutschen Bundestags arbeiten, sind die Untersuchungen nicht als idiosynkratrische Fallstudien zu verstehen, also nicht als Studien, 
die ein exklusives Erkenntnisinteresse am politischen Repräsentationsprozess des „Sonderfalls“ Deutschlands haben. Stattdessen beruht die Fallauswahl in allen Aufsätzen auf der Überzeugung, dass eine Untersuchung des deutschen Falls Erkenntnisse liefern kann, die auch auf politische Repräsentationsprozesse in anderen parlamentarischen Demokratien westlicher Prägung übertragbar sind, weil die Bundesrepublik Deutschland als ein Vertreter dieser politischen Systeme wesentliche Schlüsseleigenschaften mit ihnen teilt. Der erste Aufsatz, „Do immigrant-origin candidates attract immigrant-origin voters in party-centred electoral systems? Evidence from Germany", behandelt den deutschen Fall etwa als den eines Wahlsystems, das im Lichte einer theoretischen und vergleichenden Literatur (z.B. Carey \& Shugart, 1995; Shugart et al., 2005) geringe Anreize für kandidatenbasiertes Wahlverhalten generieren sollte. Der dritte Aufsatz, „MPs‘ Principals and the Substantive Representation of Disadvantaged Immigrant Groups“, nutzt den deutschen Fall als Beispiel einer Institutionenkombination, die Verhaltensanreize für Parlamentarier erzeugt, konfligierende Interessen $\mathrm{zu}$ repräsentieren. Erstens ist diese Eigenschaft jedoch keine Idiosynkrasie Deutschlands (siehe z.B. Martin, 2014; Preece, 2014), so dass die Ergebnisse dieser Untersuchung prinzipiell generalisierbar sein sollten. Zweitens können die Ergebnisse solcher spezifischeren Untersuchungen auch einen generalisierenden Mehrwert haben, indem sie Antworten geben können auf sogenannte „what if“ Fragen (Laver \& Shepsle, 1996, 6): welche Mechanismen sind in anderen Ländern zu erwarten, wenn zum Beispiel ein Wahlsystem nach deutschem Vorbild eingeführt wird, so wie es Anfang der 1990er Jahre in Neuseeland geschehen ist? Ähnliche Generalisierungsansprüche gelten auch für die zwei anderen Aufsätze.

Aus diesen Generalisierungsansprüchen ergibt sich das Erfordernis, das übergeordnete formale Modell in seinen Bestandteilen allgemein genug $\mathrm{zu}$ halten, so dass es von den Idiosynkrasien des empirisch untersuchten Falls zu abstrahieren vermag, um auf allgemeinere institutionelle Eigenschaften und Mechanismen abzustellen, die auch andere parlamentarische Demokratien auszeichnen (Laver \& Shepsle, 1996, 7-8). Um falsifizierbare Hypothesen deduzieren zu können, bedarf es dazu jedoch expliziter und die Realität vereinfachender Annahmen über vorherrschende Bedingungen in einer abstrakten weil generalisierbaren politischen Umwelt (Morton, 1999, 36). Das in den letzten beiden Unterabschnitten 2.1 und 2.2 entwickelte Grundmodell macht daher die Realität vereinfachende Annahmen. Zum Beispiel sind die beschriebenen Institutionen in der Realität nicht ausschließlich exogen sondern auch endogen, weil politische Akteure nicht nur in ihnen handeln sondern diese auch gestalten könnten (siehe hierzu ausführlich Diermeier \& Krehbiel, 2003). Die Annahme 
exogen gegebener Institutionen kann jedoch damit gerechtfertigt werden, dass es sich um langfristig stabile Strukturen handelt, die selten reformiert werden. Darüber hinaus sind vereinfachende Annahmen auch deshalb notwendig, weil erst durch sie relevante und falsifizierbare Hypothesen deduziert werden können: „Typically, the more general the assumptions of a model, the less clear its predictions“ (Morton, 1999, 40).

Die theoretische Modellierung von Repräsentationsprozessen basierend auf vereinfachenden Annahmen ist daher aus analytischen Gründen im Rahmen dieser Dissertation notwendig. Sie dient dazu, unmissverständliche und empirisch überprüfbare Hypothesen zu deduzieren, deren empirische Überprüfung prinzipiell auch in einem Universum potentieller Untersuchungsfälle Anwendung finden könnte. Diesem Maßstab kommt auch in den folgenden zwei Abschnitten (3. und 4.) Bedeutung zu, wenn die theoretischen Argumente im Detail diskutiert werden.

\section{Institutionelle Mechanismen deskriptiver Repräsentation in der Einwanderungsgesellschaft}

Dieser Abschnitt hat folgenden Aufbau. Zunächst leistet Unterabschnitt 3.1 eine Diskussion des Forschungsstandes, um diejenigen Forschungslücken zu identifizieren, die die ersten zwei Aufsätze dieser Dissertation bearbeiten. Unterabschnitt 3.2 erörtert dann die theoretischen Argumente der ersten beiden Aufsätze und spezifiziert die Beiträge dieser zu der existierenden Forschung. Unterabschnitt 3.3 erläutert anschließend die Datengrundlage, Fallauswahl und die methodischen Vorgehensweisen beider Aufsätze, mit denen die theoretischen Argumente empirisch überprüft werden. Der letzte Unterabschnitt 3.4 diskutiert die Befunde der empirischen Untersuchungen.

\subsection{Forschungsstand}

Eine umfangreiche Forschungsliteratur widmet sich dem Einfluss von Wahlsystemen auf die deskriptive Repräsentation von (allochthonen und autochthonen) ethnischen Minderheitsgruppen (Alba \& Foner, 2009; Barreto, 2007; Bergh \& Bjorklund, 2011a, 2011b; Bloemraad, 2013; Bloemraad \& Schönwälder, 2013; Bochsler, 2010, 2011; Dancygier, 2014; Friedman, 2005; Kostadinova, 2007; Landa et al., 1995; Lijphart, 2004; McConnaughy et al., 2010; Moser, 2008; Norris, 2004; Norris \& Lovenduski, 1995; Reilly, 2002; Reynolds, 2006; 
Ruedin, 2009, 2013; Schönwälder, 2013; Teney et al., 2010; Togeby, 2008; Trounstine \& Valdini, 2008; Zingher \& Farrer, 2016; Zollinger \& Bochsler, 2012). Der folgende Literaturüberblick verfolgt das Ziel, die Forschungsbeiträge der ersten beiden Aufsätze der vorliegenden Dissertation herauszustellen. Um einen strukturierten Überblick über diese Literatur zu geben, werden zwei Wahlsystemdimensionen unterschieden: die erste Dimension bezieht sich auf den Proportionalitätsgrad der Sitzzuteilung zwischen den Parteien (inter-party dimension) und die zweite auf die Kandidatenzentriertheit des Wahlsystems (intra-party dimension) (Shugart, 2001, 2005).

Vor allem die Wahlformel (Mehrheits- versus Verhältniswahlformel) und die Wahlkreisgröße von Wahlsystemen ${ }^{7}$ beeinflussen den Proportionalitätsgrad zwischen den Wählerstimmenanteilen von Parteien und ihren parlamentarischen Sitzanteilen (Cox, 1997; Duverger, 1951; Gallagher \& Mitchell, 2005; Lijphart, 1994). Je stärker Stimmen- und Sitzanteile korrespondieren, desto höhere Proportionalitätsgrade werden erreicht und desto geringere Hürden bestehen für Parteien mit geringen Stimmanteilen, bei der Sitzzuteilung berücksichtigt zu werden (Ezrow, 2010; Lijphart, 1999).

Da Verhältniswahlsysteme regelmäßig bessere Proportionalitätsgrade als Mehrheitswahlsysteme vorweisen können, ist ein oft $\mathrm{zu}$ findendes Argument, dass Verhältniswahlsysteme auch soziodemographische Bevölkerungsmerkmale grundsätzlich proportionaler repräsentieren als Mehrheitswahlsysteme und somit auch eine bessere deskriptive Repräsentation benachteiligter ethnischer Minderheiten gewährleisten würden (Lijphart, 2004; McLean, 1991; Ruedin, 2009). Eine Reihe jüngerer Forschungsarbeiten argumentieren jedoch, dass dieser Zusammenhang maßgeblich von der geographischen Siedlungsstruktur der untersuchten Minderheitsgruppe abhängig sei. Demnach würde die Siedlungsstruktur mit dem territorialen Aspekt von Wahlsystemen interagieren, also damit, ob Wahlsysteme eher dezentralisiert in Einpersonenwahlkreisen oder zentralisiert in einem oder wenigen Mehrpersonenwahlkreisen operieren (Bochsler, 2011; Dancygier, 2014; Donovan, 2007; Friedman, 2005; Garbaye, 2000; Kostadinova, 2007; Moser, 2008; Trounstine \& Valdini, 2008; Zollinger \& Bochsler, 2012). Die Nachteile von Einpersonenwahlkreisen würden demnach mit zunehmender geographischer Konzentration der Minderheitsgruppe abnehmen, weil diese in einer Reihe von Wahlkreisen eine beachtliche Stimmenstärke

\footnotetext{
${ }^{7}$ Obgleich der Wahlformel und der Wahlkreisgröße eine zentrale Bedeutung als Einflussfaktoren der Proportionalität zugesprochen wird, muss dennoch darauf hingewiesen werden, dass andere Wahlsystemcharakteristika ebenfalls Wirkung auf den Proportionalitätsgrad entfalten können (z.B. Malapportionment, Prozenthürden, Parlamentsgröße) (Lijphart, 1999, 143-170).
} 
erreichen könnte und dadurch bessere Möglichkeiten hätte, das stimmenmotivierte Interesse von Parteien anzuziehen und die politische Mobilisierung der eigenen Gruppe vor Ort zu organisieren (Dancygier, 2014; Garbaye, 2000; Laurence \& Maxwell, 2012; Moser, 2008; Zollinger \& Bochsler, 2012). In Verhältniswahlsystemen mit Mehrpersonenwahlkreisen hätten Parteien hingegen stärkere Anreize zum „ticket-balancing“. Damit ist die Nominierung einer weitgehend inklusiven Kandidatenliste gemeint, auf der sich alle relevanten sozialen Gruppen wiederfinden würden, um im Gegenzug die jeweiligen Parteien mit ihren Stimmen zu belohnen (Dancygier, 2014; Norris, 2004, 188-189; Norris \& Lovenduski, 1995; Reynolds, 2006, 27; Schönwälder, 2013, 643; Valdini, 2012, 741). Weil in dieser Argumentationslinie die geographische Konzentration von Minderheitsgruppen keine große Bedeutung unter den Bedingungen von Verhältniswahlsystemen beigemessen wird, folgen diese Arbeiten der weitverbreiteten Logik, dass geographische Repräsentation eine Stärke von Mehrheitswahlsystemen und eine Schwäche von Verhältniswahlsystemen sei (Latner \& McGann, 2005). Aufsatz 2 dieser Dissertation stellt insbesondere diese Logik auf die Probe, indem geographische Repräsentationsmuster unter den Bedingungen geschlossener Kandidatenlisten in Verhältniswahlsystemen analysiert werden.

Die Kandidatenzentriertheit bezieht sich hingegen vor allem auf die vom Wahlsystem ausgehenden Anreize für Wähler, ihre Wahlentscheidungen auf der Grundlage der persönlichen Eigenschaften der zur Wahl stehenden Kandidaten zu treffen (Carey \& Shugart, 1995; Norris, 2004, Kapitel 10; Shugart et al., 2005). Im Wesentlichen geht es um die Frage, inwieweit das Wahlsystem Wählern (im Gegensatz zu Parteieliten) gestattet, die Wahlwahrscheinlichkeit individueller Kandidaten zu beeinflussen. Je stärker Wähler den Wahlerfolg individueller Kandidaten beeinflussen können, desto stärkere Anreize sollten Wähler haben, die persönlichen Eigenschaften der Kandidaten im Kalkül ihrer Wahlentscheidung zu berücksichtigen (Shugart et al., 2005). In Verhältniswahlsystemen mit geschlossenen (oder starren) Kandidatenlisten sollten individuelle Kandidaten hingegen eine geringere Rollen spielen, weil Wähler im Wesentlichen zwischen Parteien auswählen bzw. den Wahlerfolg individueller Kandidaten nur beeinflussen könnten, indem sie „das Kind mit dem Partei-Badewasser ausschütten“ (Norris, 2004, 234). Je stärker Wähler jedoch die individuelle Wahlwahrscheinlichkeit von Kandidaten beeinflussen könnten, desto größer würde der kandidatenbasierte Informationsbedarf werden und umso stärker würden Wähler ihre Wahlentscheidungen kandidatenbasiert treffen (Gschwend \& Zittel, 2015; Shugart et al., 
2005). Dies träfe eher auf Verhältniswahlsysteme mit offenen bzw. flexiblen $\operatorname{Listen}^{8}$ zu, in denen Wähler Präferenzstimmen an Kandidaten innerhalb von Parteilisten verteilen können, sowie auf Mehrheitswahlsysteme in Einpersonenwahlkreisen, welche die kandidatenbasierten Informationsanforderungen an Wähler geringer halten und gleichzeitig einen größeren Spielraum lassen würden, die Wahl einzelner Kandidaten zu beeinflussen (Bowler \& Farrell, 1993; Carey \& Shugart, 1995; Norris, 2004, Kapitel 10; Schoen, 2014, 794-795; Shugart et al., 2005).

Dieser häufig angenommene Kausalzusammenhang zwischen der Kandidatenzentriertheit des Wahlsystems und kandidatenorientiertem Wahlverhalten steht empirisch jedoch insgesamt auf tönernen Füßen. Galten im britischen Mehrheitswahlsystem Kandidateneffekte auf das Wahlverhalten lange Zeit als vernachlässigbar (Gaines, 1998), präsentieren jüngere Arbeiten empirische Evidenz dafür, dass die persönlichen Merkmale der Kandidaten durchaus eine wahlentscheidende Rolle spielen können (z.B. Arzheimer \& Evans, 2012; Fisher et al., 2015; Smith, 2013). Ähnliche Effekte wurden auch aus Ländern mit offenen/flexiblen Listen in Mehrpersonenwahlkreisen berichtet (Däubler \& Bräuninger, 2016; Martin, 2010; Redmond \& Regan, 2015). Untersuchungen des deutschen Wahlsystems beschränken sich in der Regel vor allem auf die Analyse von Erststimmen generierenden Kandidatenmerkmalen (Gschwend \& Zittel, 2015; Pappi et al., 2017; aber siehe Hainmueller \& Kern, 2008). Eine ländervergleichende Umfragestudie konnte außerdem zeigen, dass stärker kandidatenzentrierte Wahlsysteme auch zu einer höheren Kenntnis von, und einer häufigeren Kontaktaufnahme mit, individuellen Kandidaten führen (Norris, 2004, Kapitel 10). Diese Literaturzusammenfassung zeigt, dass die bisherige Forschung die Möglichkeit kandidatenbasierten Wahlverhaltens in parteizentrierten Wahlsystemen weitgehend ausgeklammert hat. Daher besteht Forschungsbedarf hinsichtlich der Fragestellung, ob und unter welchen Bedingungen Kandidaten das Verhalten der Wähler auch in parteizentrierten Wahlsystemen systematisch beeinflussen können.

Diese Schlussfolgerung lässt sich auch auf die wachsende empirische Forschungsliteratur über den spezifischen Einfluss von Kandidaten mit Migrationshintergrund auf das

\footnotetext{
${ }^{8}$ In offenen (oder Quasi-) Parteilisten wird die Kandidatenreihenfolge der Sitzzuteilung nicht durch die Listenplatzierung der Parteien, sondern einzig durch Präferenzstimmen der Wähler determiniert (z.B. Finnland). In flexiblen (oder latenten) Parteilisten wird die Kandidatenreihenfolge durch die Listenplatzierungen der Parteien vorbestimmt, aber Wähler haben durch Präferenzstimmen für Kandidaten die Möglichkeit, die Reihenfolge zu ändern (z.B. Belgien). In geschlossenen Parteilisten haben Wähler nur die Möglichkeit, zwischen ganzen Parteilisten von Kandidaten zu wählen, ohne eine Möglichkeit zu haben, individuelle Kandidaten mit Präferenzstimmen zu unterstützen (z.B. Portugal) (siehe Shugart 2005, 38-44).
} 
Wahlverhalten von Bürgern mit Migrationshintergrund übertragen. In ihrer Zusammenschau zeigt diese Forschungsliteratur empirisch, dass Wähler benachteiligter Einwanderergruppen in kandidatenzentrierten Wahlsystemen tatsächlich dazu tendieren, Kandidaten mit Migrationshintergrund mit ihren Stimmen zu unterstützen (Barreto, 2007; Bergh \& Bjorklund, 2011a; Landa et al., 1995; McConnaughy et al., 2010; Teney et al., 2010; Zingher \& Farrer, 2016). Gravierende Forschungslücken bestehen jedoch hinsichtlich der Frage, ob das Wahlverhalten von Bürgern mit Migrationshintergrund in stärker parteizentrierten Wahlsystemen in ähnlicher Weise von der Präsenz von Kandidaten mit Migrationshintergrund beeinflusst wird. Dies ist das Thema des ersten Aufsatzes dieser Dissertation.

Der folgende Abschnitt legt nun die spezifischen theoretischen und empirischen Beiträge der ersten beiden Aufsätze dieser Dissertation zu den beschriebenen Forschungsständen dar.

\subsection{Theoretische Argumentation und Beiträge}

Die ersten beiden Aufsätze dieser Dissertation untersuchen die Repräsentationsbeziehung zwischen Parteien und Wählern mit Migrationshintergrund, in der Parteien Kandidatenangebote machen, welche Wähler zur Grundlage ihrer Wahlentscheidung machen. Das spezifischere Interesse gilt dabei vor allem der Beeinflussung dieser Beziehung durch das Wahlsystem und durch die Regeln und Praktiken der parteiinternen Kandidatenaufstellung (vgl. Abbildung 3).

Wie im vorigen Abschnitt dargelegt, überwiegen wahlsystemorientierte Erklärungen bezüglich der deskriptiven Repräsentation gesellschaftlich benachteiligter Einwanderergruppen. Mehrheitswahlsysteme werden vor allem mit der geographischen Repräsentation dieser Gruppen in Verbindung gebracht und kandidatenzentrierte Wahlsystemregeln mit der Stimmabgabe von Wählern mit Migrationshintergrund für Kandidaten mit Migrationshintergrund. Die beiden ersten Aufsätze dieser Dissertation zeigen jedoch, dass dabei eine wichtige Einflussgröße häufig übersehen wird: Praktiken und Regeln der parteiinternen Kandidatenaufstellung. In beiden Aufsätzen wird argumentiert, dass Repräsentationsmechanismen, die häufig mit Wahlsystemen in Verbindung gebracht werden, auch durch bestimmte Regeln der Kandidatenaufstellung hervorgerufen werden und dadurch die vermuteten Effekte von Wahlsystemen konterkarieren können. Die Vorstellung, dass geschlossenen Parteilisten in Mehrpersonenwahlkreisen keine Vorteile für geographisch 
konzentrierte Minderheiten bezogen auf ihre deskriptive Repräsentation zulassen, ist daher genauso wenig allgemeingültig wie die Annahme, dass parteizentrierte Wahlsysteme gegen ein kandidatenorientiertes Wahlverhalten von Wählern mit Migrationshintergrund sprechen.

\section{Abbildung 3: Angepasstes Grundmodell für die Analyse deskriptiver Repräsentation}

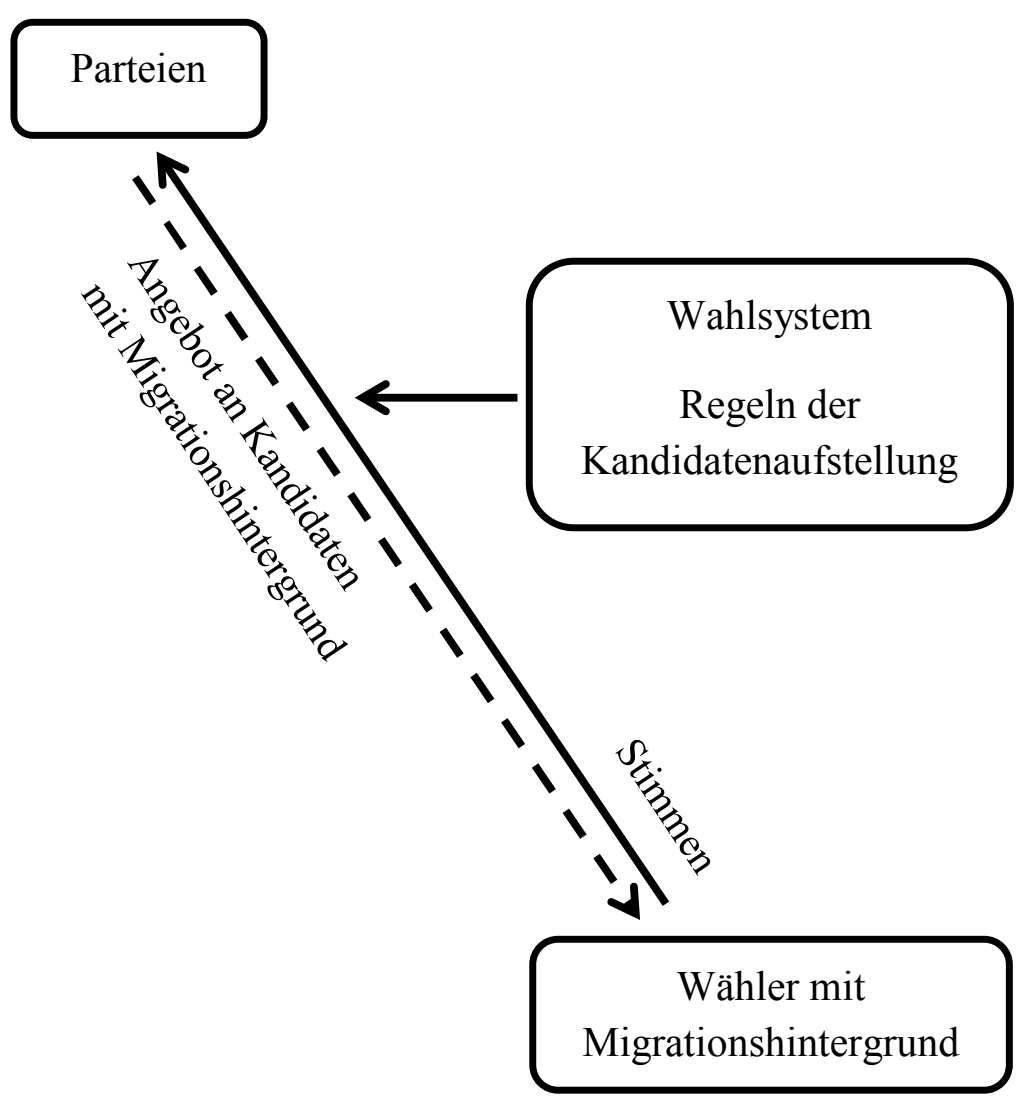

In einer Reihe von Verhältniswahlsystem mit geschlossenen Kandidatenlisten findet die Vorauswahl von Parlamentskandidaten auf der lokalen Ebene statt (Gallagher, 1988; Krouwel, 2012). Hier verlangen informelle Rekrutierungsregeln von Politikern, die eine parlamentarische Karriere anstreben, dass sie zunächst einmal Erfahrungen in der Lokalpolitik sammeln, zum Beispiel indem sie Ämter in lokalen Parteiorganisationen und/oder Mandate in Stadt- oder Gemeinderäten besetzen, bevor sie von Parteiakteuren für ein nationales Amt oder Mandat in Betracht gezogen werden. Selbst als etablierte Parlamentarier wird von ihnen erwartet, dass sie ihre lokalen Ämter und Mandate behalten, um eine Verbindung zur Lokalpolitik aufrecht zu erhalten. Tatsächlich existieren solche Rekrutierungsmuster in einer Reihe von Verhältniswahlsystemen mit geschlossenen Kandidatenlisten, etwa in Deutschland (Susanne S. Schüttemeyer \& Sturm, 2005; Wessels, 1997), Israel (Hazan, 1999), Italien (Russo, 2011), den Niederlanden (Leijenaar \& Niemöller, 1997) und Portugal (Fernandes et al., 2018). 
Kandidaten und Parlamentarier können somit lokal verwurzelt sein, wodurch Parteien eine Möglichkeit haben, diese lokalen Verbindungen für die Mobilisierung lokal definierter Wählergruppen zu nutzen, zum Beispiel um lokal konzentrierte Wähler mit Migrationshintergrund zu mobilisieren. Durch ihre lokale Verwurzelung geben individuelle Kandidaten der Partei ein „lokales Gesicht“, das das Bewusstsein der Wähler mit Migrationshintergrund vor Ort bezüglich dieser Kandidaten erhöht und somit die in den Migrationsprofilen der Kandidaten enkodierten „cues“ vermittelt, welche diese Wähler daraufhin in das Kalkül ihrer Wahlentscheidung miteinbeziehen.

Aus diesen theoretischen Überlegungen ergeben sich zwei wesentliche Konsequenzen, deren empirische Überprüfung Gegenstand jeweils eines der beiden ersten Aufsätze ist. Erstens sollten existierende Befunde bezüglich des kandidatenorientierten Wahlverhaltens von Wählern mit Migrationshintergrund nicht nur auf kandidatenzentrierte sondern auch auf die meisten parteizentrierten Wahlsysteme zutreffen, in denen parteiinterne Rekrutierungsregeln verlangen, dass Kandidaten eine lokale Verwurzelung aufbauen und pflegen. Die Kandidatenorientierungen von Wählern mit Migrationshintergrund wurden bislang jedoch nur unter Bedingungen kandidatenzentrierter Wahlsysteme untersucht, das heißt unter Bedingungen von Verhältniswahlsystemen mit offenen bzw. flexiblen Kandidatenlisten oder unter Bedingungen von Mehrheitswahlsystemen mit Einpersonenwahlkreisen, während Untersuchungen $\mathrm{zu}$ parteizentrierten Verhältniswahlsystemen mit geschlossenen Kandidatenlisten gänzlich fehlen. Der erste Aufsatz dieser Dissertation „Do immigrant-origin candidates attract immigrant-origin voters in party-centred electoral systems? Evidence from Germany" ist daher die erste empirische Untersuchung dieser Art in einem stärker parteizentrierten Wahlsystem, dem deutschen personalisierten Verhältniswahlsystem. Damit leistet dieser Aufsatz einen wichtigen Beitrag zur Schließung der aufgezeigten Forschungslücke.

Zweitens bestehen für Parteien somit auch in den meisten Verhältniswahlsystemen mit geschlossenen Kandidatenlisten elektorale Anreize, auf lokale Konzentrationen von Bürgern mit Migrationshintergrund mit der Nominierung von Kandidaten mit Migrationshintergrund zu reagieren. Parteien können ihre Kandidaten mit Migrationshintergrund als geographisch wirksame Ressource im Wahlkampf einsetzen, um in Bezirken mit hohen Migrantenanteilen für die Partei zu werben. Dabei ist der Status der Kandidaten als lokal verwurzelte Politiker, als aussichtsreiche Listenkandidaten und als möglicherweise bereits amtierende Parlamentarier von Vorteil. Parteien sollten deshalb nicht nur einen Anreiz haben Politiker 
mit Migrationshintergrund, die in diesen Bezirken verwurzelt sind, zu Kandidaten zu machen, sondern auch Anreize haben, ihnen aussichtsreiche Listenplätze zu geben, so dass sie während der Legislaturperiode als gewählte Parlamentarier das lokale Stimmenpotential von Bürgern mit Migrationshintergrund aufrechterhalten und möglicherweise sogar vergrößern können. Dadurch entstehen im Aggregat geographische Repräsentationsmuster, in denen lokale Konzentrationen von Bürgern mit Migrationshintergrund von Parlamentariern mit Migrationshintergrund repräsentiert werden.

Dies würde jedoch gegen eine strikte Auslegung der in der Wahlsystemforschung häufig implizit getroffenen Annahme sprechen, dass geographische Repräsentationsmuster vor allem in Mehrheitswahlsystemen mit Einpersonenwahlkreisen jedoch nicht in Mehrpersonenwahlkreisen mit geschlossenen Parteilisten zu finden seien (Latner \& McGann, 2005). Vermutlich aus diesem Grund mangelt es bisher an Untersuchungen geographischer Repräsentationsmuster von Bürgern mit Migrationshintergrund unter Bedingungen geschlossener Parteilisten in Mehrpersonenwahlkreisen. Der zweite Aufsatz "The more concentrated, the better represented? The geographical concentration of immigrants and their descriptive representation in the German mixed-member system?" leistet deshalb einen wichtigen weil kontraintuitiven Beitrag zu der Literatur über Wahlsystemeffekte auf die deskriptive Repräsentation benachteiligter ethnischer Minderheiten und ergänzt gleichzeitig den ersten Aufsatz durch eine Untersuchung der Bestimmungsfaktoren der Positionen von Kandidaten mit Migrationshintergrund in geschlossenen Parteilisten während der Bundestagswahl 2013.

\subsection{Fallauswahl, Daten und Methoden}

Empirisch stellen beide Aufsätze Einzelfallstudien der Bundestagswahl 2013 dar. Als Land, in dem im Jahr 2013 ungefähr 20\% aller Menschen mit Migrationshintergrund der Europäischen Union lebten (OECD \& EU, 2015), in dem ungefähr 20\% der Bevölkerung und 9\% der Wähler einen Migrationshintergrund hatten (Bundeswahlleiter, 2013) und in dem eine ausgeprägte gesellschaftliche Benachteiligung der überwiegenden Mehrheit der Bevölkerung mit Migrationshintergrund vorlag (Alba \& Foner, 2015; Die Beauftragte der Bundesregierung für Migration, Flüchtlinge und Integration, 2016), ist Deutschland ein besonders relevantes 
Land für die Erforschung der deskriptiven (und substantiellen) Repräsentation von Bürgern mit Migrationshintergrund.

Das deutsche Wahlsystem kann als gemischtes, oder personalisiertes, Verhältniswahlsystem kategorisiert werden ('mixed-member proportional' nach Shugart \& Wattenberg, 2001), in dem Wähler zwei Stimmen haben: eine Erststimme für einen Kandidaten in einem von insgesamt 299 Einpersonenwahlkreisen, in denen jeweils ein Mandat nach einfacher Mehrheitswahl vergeben wird, und eine Zweitstimme für eine geschlossene Parteiliste in einem von insgesamt 16 Mehrpersonenwahlkreisen, in denen zusammengenommen eine ähnlich hohe Zahl von Parlamentarier gewählt wird (299 plus die Anzahl an Überhangs- und Ausgleichsmandaten) (siehe auch Manow, 2015, 11-15). Trotz dieser zwei zu unterscheidenden Wahlsystemsegmente bzw. Wahlmodi (Wahlkreis- und Listenmodus) sorgt das Wahlsystem insgesamt für hohe Proportionalitätsgrade zwischen Stimmen- und Sitzanteilen für Parteien, weil die Sitzanteile der Parteien durch einen kompensatorischen Ausgleichsmechanismus zwischen den Segmenten auf Grundlage der nationalen Zweitstimmenanteile ermittelt werden (siehe auch Saalfeld, 2005).

Gemischte Wahlsysteme spielen eine wichtige Rolle in der Forschungsliteratur zum Einfluss von Wahlsystemen auf die deskriptive Repräsentation ethnischer Minderheitsgruppen. Unter der Annahme einer gegenseitigen Unabhängigkeit der beiden Wahlsystemsegmente werden sie häufig für quasi-experimentelle Vergleiche zwischen Mehrheits- und Verhältniswahlsystemregeln herangezogen (z.B. Moser, 2008). Ein Großteil der bereits beschriebenen empirischen Befunde hinsichtlich des Zusammenhangs zwischen geographischen Repräsentationsmustern und Mehrheitswahlsystemen stammen daher aus Untersuchungsdesigns in gemischten Wahlsystemen (Donovan, 2007; Friedman, 2005; Kostadinova, 2007; Moser, 2008; Wüst, 2014b; Zollinger \& Bochsler, 2012). Im Gegensatz zur bisherigen Forschungsliteratur betrachtet der zweite Aufsatz die Möglichkeit geographischer Repräsentationsmuster jedoch nicht als ein Phänomen, das auf das Mehrheitswahlsegment gemischter Wahlsysteme beschränkt ist, sondern als eines, das sich aufgrund der lokalen Verwurzelung der Kandidaten, wie im letzten Abschnitt beschrieben (siehe auch S. 4-5 in Aufsatz 2), auch im Listensegment manifestieren sollte.

Vor dem Hintergrund des beschriebenen wahlsystemorientierten Forschungsstands und den eigenen theoretischen Überlegungen könnte das Listenwahlsegment des deutschen Wahlsystems darüber hinaus auch als ein „least-likely“ (Gerring \& Seawright, 2007) Kontext für kandidatenorientiertes Wahlverhalten behandelt werden. Das Wahlverhalten bezüglich der 
Zweitstimme, welches die Sitzanteile der Parteien im Bundestag determiniert, wird weitläufig als „heavily party-oriented“ bezeichnet (Moser \& Scheiner, 2005, 259) und sollte daher (im Gegensatz zur Erststimme) geringe Anreize für kandidatenorientiertes Wahlverhalten generieren (Carey \& Shugart, 1995; Shugart et al., 2005). Der erste Aufsatz dieser Dissertation betrachtet jedoch die Möglichkeit kandidatenorientierten Wahlverhaltens als ein Phänomen, das sich aufgrund der lokalen Verwurzelung der Kandidaten, wie im letzten Abschnitt beschrieben (siehe auch S. 2-5 in Aufsatz 1), auch im Zweitstimmenwahlverhalten von Wählern mit Migrationshintergrund manifestieren sollte.

Die lokale Verwurzelung der Kandidaten gilt in Deutschland als eine wichtige Voraussetzung für eine erfolgreiche Bundestagkandidatur (z.B. Schüttemeyer \& Sturm, 2005; Wessels, 1997). Formal sieht das deutsche Wahlrecht vor, dass die parteiinterne Kandidatenauswahl in den Wahlsystemsegmenten getrennt stattfindet, d.h. Wahlkreiskandidaten werden von lokalen Parteiorganisationen in den Gebietseinheiten der Wahlkreise aufgestellt, während die Kandidatenlisten zentralisierter auf Länderebene zusammengestellt werden (Detterbeck, 2016; Roberts, 1988). Tatsächlich tritt aber die große Mehrheit der Kandidaten als sogenannte Doppelkandidaten sowohl in Wahlkreisen als auch auf den Landeslisten an (Manow, 2015; Schüttemeyer \& Sturm, 2005). Eine Nominierung als lokaler Wahlkreiskandidat gilt gemeinhin als wesentliche Voraussetzung für eine aussichtsreiche Listenposition auf Länderebene, weshalb tatsächlich ein sequenzielles Nominierungsverfahren stattfindet, in der Landeslisten erst zusammengestellt werden, nachdem Wahlkreiskandidaten bereits nominiert wurden (Detterbeck, 2016; Manow, 2013; Reiser, 2014; Schüttemeyer \& Sturm, 2005). De facto kann man daher auch im Listensegment des deutschen Wahlsystems von einem dezentralisierten Nominierungsverfahren sprechen.

Durch die Kombination eines gemischten Wahlsystems mit einer dezentral organisierten Kandidatennominierungspraxis ist das deutsche politische System daher ein geeigneter Untersuchungsfall, um die im letzten Abschnitt identifizierten Forschungslücken zu untersuchen.

Dafür nutzen beide Aufsätze einen Datensatz aller 1493 Wahlkreiskandidaten, die in der Bundestagswahl 2013 für CDU/CSU, SPD, Grüne, FDP oder Linke angetreten sind, als gemeinsame Datenbasis. Der Datensatz wurde auf Anfrage von den Administratoren der Internetseite www.abgeordnetenwatch.de zur Verfügung gestellt und vom Autor dieser Dissertation mit Unterstützung von studentischen Hilfskräften validiert, erweitert und aufbereitet. Von zentralem Interesse waren dabei die Kandidaten, die selbst einen 
Migrationshintergrund haben (siehe S. 6-7 in Aufsatz 1, bzw. S. 6 in Aufsatz 2). Beide Aufsätze nutzen darüber hinaus unterschiedliche Datenquellen, die unabhängig voneinander mit dem Kandidatendatensatz auf Wahlkreisebene verknüpft wurden.

Im Fall des ersten Aufsatzes handelt es sich dabei um Wahlumfragedaten der German Longitudinal Election Study (GLES 2013) (Rattinger et al., 2014). Dieser Wahlumfragedatensatz bietet die Möglichkeit, Wähler mit und ohne Migrationshintergrund sowie ihre jeweilige Wahlkreiszugehörigkeit zu identifizieren. Auf dieser Grundlage konnten Wähler und Wahlkreiskandidaten der beiden Datensätze, jeweils mit und ohne Migrationshintergrund, auf der Ebene von Wahlkreisen miteinander verknüpft werden, wobei sich die Analyseeinheit durch die Individualdaten der Wähler definiert. Durch diese innovative Forschungsperspektive, die Kandidaten- und Wählerinformationen auf der Individualebene verknüpft (für ein ähnliches Untersuchungsdesign siehe Gschwend \& Zittel, 2015), kann im Vergleich zu einem auf Aggregatdaten basierenden Forschungsdesign die Gefahr eines ökologischen Fehlschlusses weitgehend ausgeschlossen werden. Die abhängige Variable ist definiert als die Wahlentscheidung der Wähler (Zweitstimme). Die zentrale unabhängige Variable ist die lokale Präsenz von Kandidaten mit bzw. ohne Migrationshintergrund. Bezogen auf die abhängige Variable beinhaltet die Datenstruktur daher sowohl optionsspezifische Variation hinsichtlich der zur Wahl stehenden Parteien (Eigenschaften des lokalen Kandidaten von Partei A, Eigenschaften des lokalen Kandidaten von Partei B, usw.) als auch wählerspezifische Variation bezogen auf die Eigenschaften des Wählers (z.B. die Links-Rechts-Selbsteinstufung als Kontrollvariable). Aufgrund der vorliegenden Datenstruktur bietet sich daher für die empirische Analyse eine konditionale logistische Regression an, welche es erlaubt, das Resultat multinominaler Entscheidungssituationen (Zweitstimmenwahlentscheidung) als ein durch optionsspezifische und wählerspezifische Variation beeinflusstes Phänomen zu analysieren (Alvarez \& Nagler, 1998; siehe auch S. 10-11 in Aufsatz 1).

Im Fall des zweiten Aufsatzes wurden die Kandidatendaten mit wahlkreisspezifischen Bevölkerungs- und Wahlergebnisdaten verknüpft. Der Datensatz über die Wahlkreisstrukturen wurde im Rahmen des PATHWAYS ${ }^{9}$ Projekts gesammelt und für die im

\footnotetext{
${ }^{9}$ Der Verfasser dieser Dissertation sowie seine Koautoren sind Teil des Bamberger Forscherteams im Projekt 'Pathways to Power: The Political Representation of Citizens of Immigrant Origin in Seven European Democracies (PATHWAYS)'. Das Projekt wurde finanziert durch den ANR (Frankreich), die DFG (Deutschland), den ESRC (Großbritannien) und die NWO (Niederlande) im Rahmen der Open Research Area (ORA+). Das PATHWAYS Konsortium besteht aus der University of Amsterdam (Professor Dr. Jean Tillie), der Otto-Friedrich-
} 
zweiten Aufsatz präsentierte statistische Analyse ebenfalls mit den Kandidatendaten auf der Ebene von Wahlkreisen verknüpft. Die abhängige Variable der Untersuchung bezieht sich dabei auf das Rekrutierungsverhalten der Parteien, das heißt genauer auf die Positionierung der Kandidaten in den Parteilisten des Listenwahlsystemsegments. Die unabhängigen Variablen beziehen sich im Wesentlichen auf den Migrationshintergrund der Kandidaten sowie auf den prozentualen Bevölkerungsanteil von Bürgern mit Migrationshintergrund ${ }^{10}$ in den Wahlkreisen der Kandidaten. Für die quantitative Überprüfung des Zusammenhangs zwischen den unabhängigen Variablen und den Listenpositionen der Kandidaten wurde ein Tobit-Regressionsmodell herangezogen, mit dem die nicht-lineare Abnahme der Wahlerfolgsaussichten von Positionen am unteren Ende der Landeslisten kontrolliert werden kann (Long, 1997, 187; siehe auch S. 7-8 in Aufsatz 2).

\subsection{Empirische Befunde}

Die Ergebnisse der quantitativen Analysen lassen insgesamt nicht den Schluss zu, dass die Mechanismen der deskriptiven Repräsentation von Bürgern mit Migrationshintergrund gängigen Wahlsystemargumenten entsprechen. Stattdessen erfahren die vorgeschlagenen Argumente empirische Unterstützung, welche die parteiinterne Rekrutierungspraxis bezogen auf das Erfordernis einer lokalen Verwurzelung der Kandidaten in den Vordergrund stellen.

Erstens sprechen die empirischen Ergebnisse aus Aufsatz 1 nicht für das Argument, dass kandidatenzentrierte Wahlsysteme eine notwendige Bedingung für kandidatenorientiertes Wahlverhalten sind. Es zeigt sich stattdessen, dass das Zweitstimmenwahlverhalten von Wählern mit Migrationshintergrund während der Bundestagswahl 2013 tatsächlich von der Präsenz lokaler Kandidaten mit Migrationshintergrund beeinflusst wurde. Wenn sich Wähler mit Migrationshintergrund der Präsenz lokaler Kandidaten mit Migrationshintergrund bewusst waren, dann erhöhte sich die Wahrscheinlichkeit auch deutlich, die Parteien dieser Kandidaten zu wählen. Eine Einschränkung besteht allerdings darin, dass sich dieser Zusammenhang auf die Parteien des linken Parteienspektrums (SPD, Linke und Grüne) zu beschränken scheint. Wenn ein Bewusstsein für einen lokalen Kandidaten mit

\footnotetext{
Universität Bamberg (Professor Dr. Thomas Saalfeld), der University of Leicester (Professor Laura Morales, PhD) und der CEVIPOF-Sciences Po Paris (Professor Dr. Manlio Cinalli).

${ }^{10}$ Aufgrund der Nichtverfügbarkeit soziodemographischer Wahlkreisdaten bezogen auf Wähler mit Migrationshintergrund musste diese Größe jedoch mit dem Ausländeranteil im Wahlkreis approximiert werden (siehe auch S. 7 sowie S. 11 in Aufsatz 2).
} 
Migrationshintergrund der SPD, Linken, oder Grünen vorlag, dann erhöhte sich die Wahrscheinlichkeit einer Zweitstimmenabgabe für diese Parteien um 0,41, 0,67 und 0,63 Prozent (vgl. S. 15 in Aufsatz 1). Demgegenüber zeigt das Bewusstsein einer Präsenz von Kandidaten mit Migrationshintergrund von Parteien rechts der Mitte (CDU/CSU, FDP) keine eindeutigen Ergebnisse.

Zweitens lässt die empirische Analyse in Aufsatz 2 den Schluss $\mathrm{zu}$, dass sich das Rekrutierungsverhalten von Parteien reziprok zu den empirischen Ergebnissen des ersten Aufsatzes verhält. Kandidaten mit Migrationshintergrund erreichen umso bessere Listenpositionen, je höher der Anteil an Wählern mit Migrationshintergrund in ihren Heimatbezirken ist. Dieser Befund eines geographischen Repräsentationsmusters zeigt, dass der Vorteil einer geographischen Konzentration ethnischer Minderheiten nicht nur, wie üblicherweise angenommen, auf Mehrheitswahlsysteme zutrifft, sondern auch auf geschlossene Kandidatenlisten in Mehrpersonenwahlkreisen. Außerdem spricht der Befund dafür, dass Parteien sich der Tauschbeziehung zwischen Stimmen und Kandidaten mit Migrationshintergrund bewusst sind. Ähnlich wie in Aufsatz 1 zeigt sich allerdings, dass der geographische Zusammenhang wesentlich auf dem Rekrutierungsverhalten der Parteien des linken politischen Spektrums basiert.

Die Befunde einer politischen Linksneigung dieser Repräsentationsbeziehung, die jeweils in beiden Aufsätzen offenbar wurde, sind im Lichte der bereits existierenden Literatur allerdings wenig verwunderlich. Erstens haben frühere Untersuchungen bereits etabliert, dass die Neigung von Wählern mit Migrationshintergrund, linke Parteien zu unterstützen, ähnlichen Kalkülen unterliegt wie die Neigung, Kandidaten mit minderheitsspezifischen Merkmalen zu unterstützen. Dies scheint auch in dem hier untersuchten Zeitraum der Fall zu sein. Die gängige Interpretation dieser Zusammenhänge beruht auf der Annahme einer wahrgenommenen Schicksalsgemeinschaft, in der eine Verbesserung der eigenen Position nur durch eine Verbesserung der Gruppensituation insgesamt erreicht werden kann (Dancygier \& Saunders, 2006; Sanders et al., 2014). Wähler mit Migrationshintergrund würden daher dazu tendieren, Parteien links der politischen Mitte zu wählen, weil die inhaltlichen Positionen dieser Parteien eher im Einklang mit einer Verbesserung der Gruppensituation stehen als die Positionen der Parteien rechts der Mitte. In den Worten Anthony Messinas:

"the left's traditional policy agenda tends to dovetail with the perceived interests of immigrants. Specifically, the proclivity of the left to privilege the goals of full employment, greater socioeconomic equality, universal health care, a vibrant public 
educational system, racial equality and so on intersects with the material and subjective aspirations of immigrant voters who generally find themselves socioeconomically disadvantaged or the objects of racial prejudice or social exclusion" (Messina, 2007, 208).

Wenn jedoch ein Kandidat mit Migrationshintergrund für eine Partei des rechten Parteispektrums kandidiert, dann sollten die in den Parteien- und Kandidatenprofilen enkodierten „cues“ sich gegenseitig aufheben (siehe dazu ausführlicher S. 3-4 in Aufsatz 1). Parteien des rechten Parteispektrums sollten deswegen auch geringere elektorale Anreize haben, entsprechende Kandidatenangebote zu machen. Darüber hinaus existiert empirische Evidenz aus Umfrageexperimenten, die zeigt, dass Kandidaten mit Migrationshintergrund von traditionellen deutschstämmigen Wählern des rechten Parteispektrums tendenziell elektoral diskriminiert werden (Street, 2014). Dies sollte die elektoralen Anreize für Parteien rechts der Mitte noch weiter abschwächen, Kandidaten mit Migrationshintergrund aufzustellen.

Trotz der wichtigen Forschungsbeiträge, die beide Aufsätze leisten, haben die empirischen Analysen jedoch auch erwähnenswerte Limitationen, die beide Aufsätze offen diskutieren (siehe S. 16-17 in Aufsatz 1 und S. 11-12 in Aufsatz 2). Erstens besteht eine Limitation hinsichtlich der Generalisierbarkeit der empirischen Befunde aufgrund der Beschränkung der empirischen Analyse auf ein einzelnes Land, das sich durch ein spezifisches Wahlsystem auszeichnet. Dadurch bleibt vor allem die Frage offen, inwieweit die Befunde aus der Analyse eines gemischten Wahlsystems auch auf „reine“ Verhältniswahlsysteme mit geschlossenen Kandidatenlisten übertragbar sind. Zweitens gibt es Limitationen, die sich aus der Datenverfügbarkeit ergeben. So war es leider nicht möglich, den Einfluss spezifischer Migrationshintergründe, sowohl auf Seiten der Kandidaten als auch auf Seiten der Wähler, zu isolieren. In Aufsatz 2 konnte dadurch nur der Zusammenhang zwischen dem lokalen Ausländeranteil und den Listenpositionen von Kandidaten als Träger des allgemeinen Merkmals „Migrationshintergrund“ empirisch getestet werden. Es konnte aber leider nicht getestet werden, ob dieser Zusammenhang nur für spezifische Migrationshintergründe existiert (z.B. nur für Wähler und Kandidaten mit türkischem Migrationshintergrund). Dies gilt in ähnlicher Weise auch für Aufsatz 1, in dem aufgrund geringer Fallzahlen von Wählern mit Migrationshintergrund der Zusammenhang nur für das allgemeine Merkmal „Migrationshintergrund“" auf Seiten von Wählern und Kandidaten getestet werden konnte.

Diese Einschränkungen bilden jedoch gleichzeitig die Grundlage für Vorschläge, die beide Aufsätze mit Blick auf nachfolgende Forschungsvorhaben machen. Insbesondere wird 
vorgeschlagen, feingliedrigere und länderübergreifende Daten auf der Ebene von Kandidaten, Wählern und lokalen Gebietseinheiten zu erheben und zu analysieren, um die identifizierten Forschungslücken weiter zu verengen (siehe S. 17-18 in Aufsatz 1 und S. 12-13 in Aufsatz 2). Dadurch sollten beide Aufsätze zu wichtigen Referenzpunkten in zukünftigen Forschungsarbeiten über die deskriptive Repräsentation von Bürgern mit Migrationshintergrund werden.

4. Institutionelle Mechanismen substantieller Repräsentation in der Einwanderungsgesellschaft

Dieser Abschnitt ist wie folgt aufgebaut. Unterabschnitt 4.1 gibt einen Überblick über den Forschungsstand und identifiziert Forschungslücken, welche die beiden letzten Aufsätze dieser Dissertation bearbeiten. Unterabschnitt 4.2 erörtert dann die theoretischen Überlegungen und Hypothesen der beiden Aufsätze, bevor Unterabschnitt 4.3 die Datengrundlagen, Fallauswahl und die methodischen Vorgehensweisen erklärt. Der letzte Unterabschnitt 4.4 diskutiert abschließend die Befunde der empirischen Untersuchungen.

\subsection{Forschungsstand}

Die Erforschung des migrations- und integrationsspezifischen Repräsentationsverhaltens individueller Parlamentarier hat sich bis dato hauptsächlich auf die Präsenz von Parlamentariern mit Migrationshintergrund konzentriert. Im Sinne des übergeordneten theoretischen Bezugsrahmens dieser Dissertation wird in dieser analytische Perspektive vor allem die ex-ante Kontrolle der Wähler hervorgehoben, die bestimmte Repräsentationsagenten auswählen, die dann eigene intrinsische Motive verfolgen (Mansbridge, 2003, 2009). Parlamentarisches Verhalten wird als Resultat der individuellen politisch-inhaltlichen Ziele von Parlamentariern verstanden, welche in einem Zusammenhang mit den persönlichen Lebenserfahrungen der Parlamentarier stehen (Bäck et al., 2014; Blomgren \& Rozenberg, 2012; Searing, 1991). Diese Perspektive lässt vermuten, dass die Präsenz von Parlamentariern mit Migrationshintergrund positive Effekte auf die substantielle Repräsentation von Einwanderergruppen hat. Die empirische Forschungsliteratur tendiert in der Tat dazu, diesen Zusammenhang zu bestätigen: Parlamentarier mit Migrationshintergrund, 
vor allem solche, die einen „sichtbaren“ Migrationshintergrund haben, stellen häufiger schriftliche parlamentarische Fragen als andere Parlamentarier hinsichtlich migrationsspezifischer Themen und Themen der gesellschaftlichen Benachteiligung von Bürgern mit Migrationshintergrund (Aydemir \& Vliegenthart, 2016; Geese et al., 2015; Saalfeld, 2011; Saalfeld \& Bischof, 2013). Dieses Verhalten scheint sich auch nicht gegen Ende der parlamentarischen Laufbahn abzuschwächen (Wüst, 2014a). Ähnliche Befunde wurden auch aus Untersuchungen parlamentarischer Reden berichtet (Bird, 2011; Blätte \& Wüst, 2017). In ihrer Zusammenschau bestätigen diese Arbeiten daher die Vermutung, dass deskriptive Repräsentanten benachteiligter sozialer Gruppen in der Tendenz auch eine stärkere intrinsische Motivation haben, die spezifischen Interessen dieser Gruppen zu repräsentieren (Mansbridge, 1999; Phillips, 1995, 1998).

In Relation zum Forschungsstand über die Rolle von Parlamentariern mit Migrationshintergrund gibt es jedoch ein ausgeprägtes Forschungsdefizit bezüglich des Repräsentationsverhaltens von Parlamentariern unabhängig von ihrem eigenen Status als Einwanderer oder Nachfahren von Eingewanderten. Dadurch, dass Bürger mit Migrationshintergrund in den meisten westeuropäischen Parlamenten deskriptiv unterrepräsentiert sind, sollten Parlamentarier ohne Migrationshintergrund jedoch eine wichtige Rolle für die substantielle Repräsentation benachteiligter ${ }^{11}$ Einwanderergruppen spielen. Aufsatz 3 beabsichtigt diese Lücke zu verengen, indem er Parlamentarier, unabhängig vom Vorliegen eines Migrationshintergrundes, zu einem expliziten Fokus der Untersuchung macht.

Somit wird der Blick auf eine analytische Perspektive gelenkt, die migrations- und integrationsspezifisches Repräsentationsverhalten im Sinne einer Antizipation der ex-post Kontrolle der Repräsentationsagenten modelliert, das heißt als strategische Reaktionen der Parlamentarier auf die potentiellen Sanktionen ihrer Prinzipale. Diese Perspektive hebt vor allem die karriererelevanten Ziele von Parlamentariern hervor, die im Wesentlichen die Möglichkeit zukünftiger Parlamentskandidaturen, die erneute Wiederwahl und die Besetzung

\footnotetext{
${ }^{11}$ Wie im Einleitungstext zu diesem Rahmenpapier bereits erläutert wird, sind Gesellschaftsteile, die gesellschaftlich benachteiligt sind, von besonderer normativer Relevanz. Daher spielen benachteiligte Einwanderergruppen im dritten Aufsatz auch die Rolle der zu repräsentierenden Zielgruppe. Es soll damit jedoch nicht behauptet werden, dass alle Bürger mit Migrationshintergrund gesellschaftlich benachteiligt sind. Informationen darüber, welche Bürger mit Migrationshintergrund gesellschaftlich benachteiligt sind, können jedoch nicht auf der Grundlage bestimmter Merkmale, etwa durch Kenntnis der Herkunftsländer (zu deren Anteilen im Übrigen auch keine statistischen Informationen auf Wahlkreisebene vorliegen), rückgeschlossen werden. Nichtsdestotrotz ist es kein Geheimnis, dass die Meisten der in Deutschland und Westeuropa lebenden Bewohner mit Migrationshintergrund gesellschaftlich benachteiligt sind, wie im Einleitungstext dieses Rahmenpapiers bereits beschrieben wurde.
} 
politisch einflussreicher und prestigeträchtiger Parlamentsämter ${ }^{12}$ umfassen (Strøm, 1997). Weil die Erreichung dieser Ziele von den Prinzipalen effektiv verhindert werden kann, sollten Parlamentarier daher Verhaltensanreize haben, im Interesse ihrer Prinzipale zu handeln (siehe S. 15-17 in diesem Rahmenpapier). Existierende Arbeiten in dieser Forschungstradition analysieren insbesondere den Einfluss von Wahlsystemregeln (Carey, 2009; Carey \& Shugart, 1995; Mitchell, 2000; Sieberer, 2010), Regeln der Kandidatenaufstellungsverfahren (Fernandes et al., 2019; Hazan, 1999; Karlsen \& Narud, 2013; Müller, 2000; Preece, 2014) sowie parlamentsinternen Organisationsstrukturen (Carey, 2009; Martin, 2014; Saalfeld \& Strøm, 2014) auf parlamentarisches Verhalten (weitere Informationen folgen unter 3.2).

Forschungsbedarf besteht im Lichte dieser Literatur in zweierlei Hinsicht. Erstens bezogen auf die Frage, welche Prinzipale eine größere Rolle spielen, wenn die genannten Institutionen in ihrer Kombination Verhaltensanreize generieren, gegensätzliche Interessen zu repräsentieren, so dass Parlamentarier die Interessen eines Prinzipals über die eines anderen stellen müssen. Wenden sich Parlamentarier zum Beispiel eher den Interessen nationaler Parteieliten oder denen lokaler Wählergruppen bzw. lokal organisierter Parteimitgliedern zu, wenn ein parteizentriertes Wahlsystem mit einem territorial ausdifferenzierten Kandidatennominierungsverfahren kombiniert wird? Die bisherige Forschungsliteratur ist sich ob dieser gegenläufigen Einflüsse bezogen auf das Repräsentationsverhalten der Parlamentarier noch uneins. Während einige Autoren vor allem die Rolle von Wahlsystemen unterstreichen (Carey \& Shugart, 1995; Shugart et al., 2005), argumentieren andere theoretisch und empirisch fundiert, dass Kandidatennominierungsverfahren und parlamentsinterne Institutionen Wahlsystemeffekte konterkarieren könnten (Hazan, 1999; Martin, 2014; Preece, 2014). Letztere Perspektive wird auch von einem Aufsatz gestützt, den der Verfasser dieser Dissertation gemeinsam mit Jorge M. Fernandes und Carsten Schwemmer publiziert hat (Fernandes et al., 2019). Zweitens fehlt es an Untersuchungen darüber, inwieweit die substantielle Repräsentation der Interessen von gesellschaftlich benachteiligten Gruppen, z.B. Einwanderergruppen, durch eine konfligierende Kombination der genannten politischen Institutionen strukturiert wird. Der dritte Aufsatz dieser Dissertation intendiert daher, eine Brücke zwischen den Forschungsliteraturen zur substantiellen Repräsentation benachteiligter Einwanderergruppen und zur Responsivität von Parlamentariern gegenüber den konkurrierenden Erwartungen ihrer Prinzipale zu schlagen, um das wissenschaftliche Verständnis beider Phänomene zu verbessern.

\footnotetext{
12 Zum Beispiel Ausschussmitgliedschaften und -vorsitze, Mitgliedschaften im Parlamentspräsidium und Fraktionsvorständen, sowie Positionen als parlamentarische Staatssekretäre, Geschäftsführer oder Obleute.
} 
Institutionen beeinflussen Parlamentarier allerding nicht nur im Sinne von Verhaltensanreizen, sondern entfalten auch eine Wirkung auf parlamentarisches Verhalten, indem sie Parlamentariern institutionelle Schranken setzen (z.B. North, 1990). Vor allem mit Blick auf die Möglichkeit, Reden im Plenum zu halten, werden Parlamentariern in den meisten westeuropäischen Parlamenten von Geschäftsordnungen und Fraktionen enge Handlungsspielräume gesetzt, die darüber entscheiden, wer sich wann zu welchen Themen im Plenum äußern darf (Bäck \& Debus, 2016; Cox, 2006; Keh, 2015; Proksch \& Slapin, 2015). In diesem Zusammenhang hat sich eine noch junge aber wachsende Forschungsliteratur etabliert, welche die Auswirkungen dieser institutionellen Einschränkungen auf das individuelle Redeverhalten von Parlamentariern zum Gegenstand des Forschungsinteresses macht (Bäck et al., 2014; Bäck \& Debus, 2016, 2018; Debus \& Bäck, 2014; Proksch \& Slapin, 2015, 2012). Die wenigen Arbeiten, die es zum Redeverhalten von Parlamentariern hinsichtlich migrations- und integrationsrelevanter Inhalte gibt (Bird, 2011; Blätte \& Wüst, 2017), tendieren jedoch bislang dazu, die Kontrolle der Fraktionsführungen über den Zugang zum Rednerpult als Determinante auszublenden. Dies stellt eine weitere wichtige Forschungslücke dar, die die vorliegende Dissertation identifiziert und mit Aufsatz $4 \mathrm{zu}$ verengen sucht.

Die Analyse parlamentarischer Reden auf migrationsrelevante Inhalte steht darüber hinaus auch in Verbindung zu einer Forschungsliteratur, welche die Aufmerksamkeit und Positionierung politischer Akteure hinsichtlich migrations- und integrationspolitischer Themen insgesamt untersucht. Da europäische Gesellschaften vor immer größeren migrations- und integrationsspezifischen Herausforderungen stehen, spielen diese Themen auch eine immer größer werdende Bedeutung für den Parteienwettbewerb und in den Köpfen der Wähler, unabhängig davon, ob sie einen Migrationshintergrund haben oder nicht. Die politikwissenschaftliche Analyse migrationspolitischer Inhalte ist daher freilich nicht nur von Relevanz bezogen auf benachteiligte Einwanderergruppen, sondern auch mit Blick auf das Elektorat insgesamt. Während migrationsbezogene politische Einstellungen von Wählern relativ gut erforscht sind, ist jedoch weit weniger darüber bekannt, inwieweit migrationspolitische Wählerpräferenzen auch für das Handeln politischer Entscheidungsträger eine Rolle spielen: „past research has treated immigration attitudes as an end point in themselves and has not considered their influence on policy making“ (Hainmueller \& Hopkins, 2014, 227). Erste Untersuchungen haben sich seitdem hauptsächlich auf migrationspolitische Themenaufmerksamkeiten (Green-Pedersen \& Otjes, 2017; Kortmann \& Stecker, 2019), inhaltliche Positionen (Ruedin \& Morales, 2017) und das migrationspolitische 
Framing (Helbling, 2014) politischer Parteien konzentriert. Die Rolle individueller Parlamentarier ist in diesem Zusammenhang jedoch insgesamt unterbelichtet geblieben. Durch eine Untersuchung migrationsspezifischer Inhalte in parlamentarischen Reden, die für Parteien und Parlamentarier öffentlichkeitswirksame Möglichkeiten bieten, Themenprioritäten und Positionen mit Wählern zu kommunizieren (Bächtiger, 2014), leistet der vierte Aufsatz daher auch einen Beitrag zu dieser Literatur.

\subsection{Theoretische Argumentation und Beiträge}

Aufsätze 3 und 4 dieser Dissertation beschäftigen sich mit dem migrations- und integrationspolitischen Verhalten individueller Parlamentarier. Dem theoretischen Grundmodell aus Abschnitt 2.2 folgend, betrachten beide Aufsätze Parlamentarier als Akteure, die in ein Netz unterschiedlicher Erwartungen von Wählern und Parteiakteuren eingebettet sind (vgl. Abbildung 4). Unter Verweis auf Abschnitt 2.3 wird an dieser Stelle noch einmal darauf hingewiesen, dass die theoretischen Argumente in diesem Abschnitt auf vereinfachenden generalisierten Annahmen beruhen, um nicht nur auf den deutschen Bundestag als Untersuchungsfall anwendbar $\mathrm{zu}$ sein, sondern auch auf vergleichbare parlamentarische Demokratien. Aufsatz 4 fokussiert dabei vor allem auf den Einfluss institutioneller Schranken auf das Redeverhalten individueller Parlamentarier, während Aufsatz 3 den Einfluss institutioneller Verhaltensanreize auf das Frageverhalten individueller Parlamentarier untersucht.

Genauer gesagt untersucht der dritte Aufsatz „MPs' Principals and the Substantive Representation of Disadvantaged Immigrant Groups" welche institutionellen Verhaltensanreize Parlamentarier dazu veranlassen, die Interessen benachteiligter Bürger mit Migrationshintergrund substantiell zu repräsentieren. Die theoretische Argumentation geht von drei wichtigen karriererelevanten Zielen der Parlamentarier aus, die aufeinander aufbauen und daher in einer hierarchischen Reihenfolge stehen: zunächst müssen Parlamentarier erreichen, dass sie erneut als Kandidaten aufgestellt werden. Die Kandidatenaufstellung ist daher eine notwendige Bedingung für die Erreichung des zweiten Ziels der Wiederwahl, das wiederum eine notwendige Bedingung für das dritte Ziel ist, politisch einflussreiche und prestigeträchtige Ämter im Parlament zu bekleiden (Sieberer \& Müller, 2017; Strøm, 1997). Die Erreichung des ersten Ziels wird durch Parteiakteure außerhalb des Parlaments in 
Nominierungskonferenzen bestimmt (Hazan \& Rahat, 2010; Müller, 2000). Das zweite Ziel wird nur erreicht, wenn auch ausreichend Wähler den Kandidaten bzw. seine Wahlliste unterstützen (Mitchell, 2000). Und das Ziel einfluss- und prestigereicher Ämter innerhalb des Parlaments obliegt der Kontrolle der Fraktionsführungen (Carey, 2009). Wie groß die Kontrolle der Prinzipale über den Zugang zu diesen Ressourcen ist, sollte jedoch von der exogen gegebenen institutionellen Umwelt abhängen, hier Wahlsystemregeln, Regeln der parteiinternen Kandidatenaufstellung und der parlamentsinternen Organisationsstruktur (vgl. Abbildung 4).

Abbildung 4: Angepasstes Grundmodell für die Analyse substantieller Repräsentation

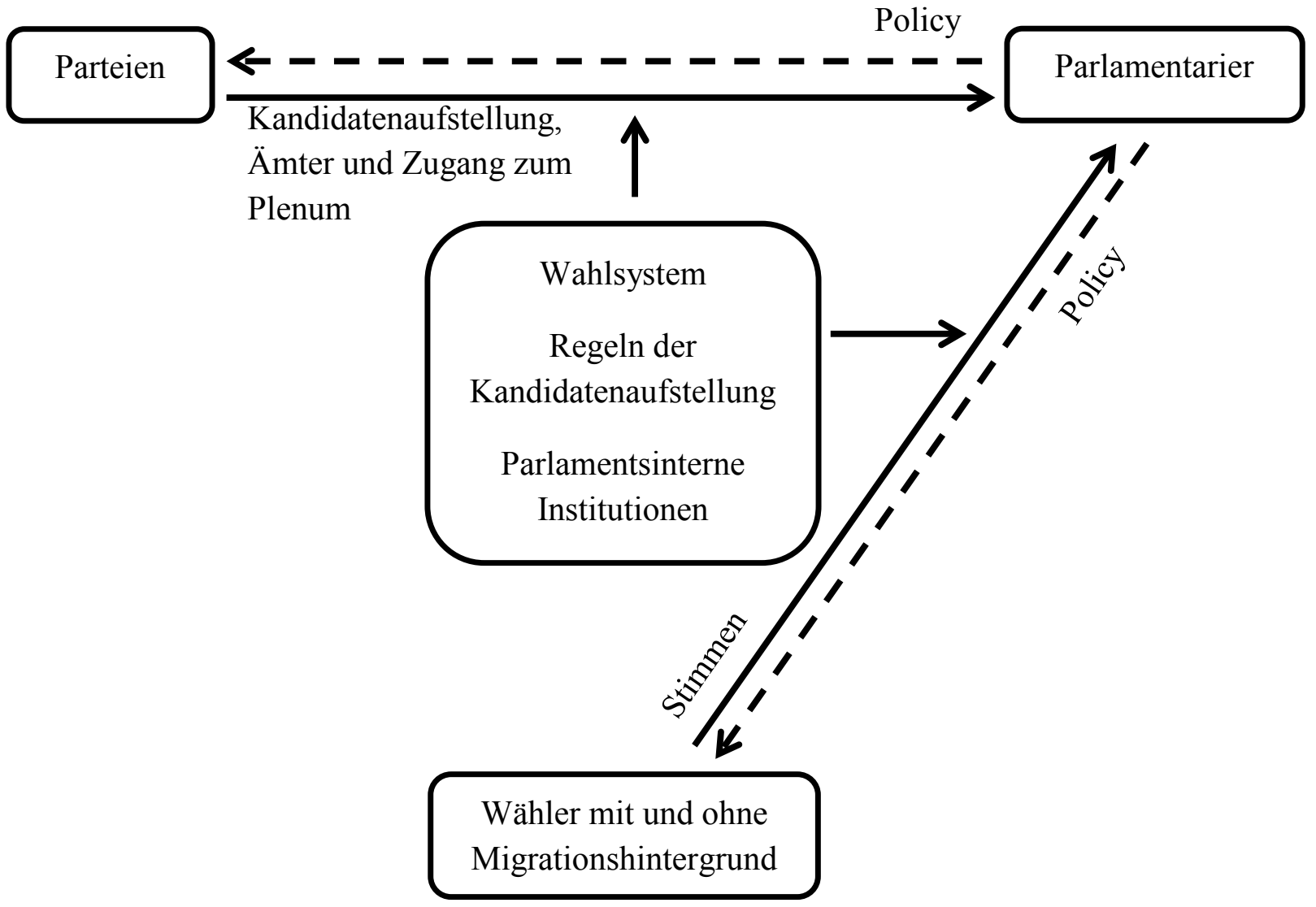

Die Regeln des Wahlsystems beeinflussen ob Wähler oder nationale Parteieliten eine relativ stärkere Kontrolle über die Wiederwahl individueller Parlamentarier ausüben können. Präziser formuliert: die Kandidatenzentriertheit des Wahlsystems (siehe S. 23-25 in diesem Rahmenpapier) beeinflusst, welche der von Wählern und Parteieliten kontrollierten Ressourcen (Wahl vs. Nominierung) für das Ziel der Wiederwahl schwerer wiegen (Carey, 2009; Carey \& Shugart, 1995; Mitchell, 2000; Norris, 2004, Kapitel 10; Shugart et al., 2005; 
Zittel \& Gschwend, 2008). In kandidatenzentrierten Wahlsystemen, z.B. in Mehrheitswahlsystemen mit Einpersonenwahlkreisen oder Verhältniswahlsystemen mit offenen/flexiblen Kandidatenlisten, sollten lokal definierte Wählergruppen eine wichtigere Rolle im Repräsentationskalkül von Parlamentariern spielen, weil diese die Wahlwahrscheinlichkeiten individueller Kandidaten direkt beeinflussen können. Parteizentrierte Wahlsysteme mit geschlossenen Kandidatenlisten generieren hingegen vor allem Anreize, den Erwartungen von Parteiakteuren zu entsprechen, weil die von diesen Akteuren in der Kandidatenauswahl bestimmten Listenpositionen effektiv über die Wiederwahl einzelner Parlamentarier vorentscheiden. Wähler haben hingegen nur die Möglichkeit, zwischen Parteien auszuwählen und können dadurch den Wahlerfolg individueller Kandidaten nur beeinflussen, indem sie „das Kind mit dem Partei-Badewasser ausschütten“ (Norris, 2004, 234). Das lokale Mobilisierungspotential von Wählern mit Migrationshintergrund sollte daher vor allem unter kandidatenzentrierten Wahlsystemregeln ein wesentlicher Bestimmungsfaktor der substantiellen Repräsentation von benachteiligten Einwanderergruppen durch individuelle Parlamentarier sein. Auf der anderen Seite sollte die migrations- und integrationspolitische inhaltliche Schwerpunktsetzung der Partei auf gesamtstaatlicher Ebene ein stärkerer Bestimmungsfaktor unter den Bedingungen von parteizentrierten Wahlsystemregeln sein.

In ähnlicher Weise kann argumentiert werden, dass, je nachdem wie zentralisiert die Kandidatenauswahl (Rahat \& Hazan, 2001) stattfindet, Parlamentarier einen Anreiz haben sollten, lokal definierte Parteiakteure stärker als gesamtstaatlich definierte Parteiakteuren zu repräsentieren. Lokale Auswahlgremien sollten vor allem ein Interesse an der Pflege der lokalen Reputation ihrer Parlamentarier haben, während Akteure in nationalen Parteizentralen vor allem die gesamtstaatliche Reputation der Partei hervorheben sollten (Gallagher \& Marsh, 1988; Karlsen \& Narud, 2013). Wenn lokale Parteien über zukünftige Kandidaturen entscheiden, dann halten diese auch ein wichtiges Mittel in den Händen um nationale Parlamentarier für lokale Belange zu nutzen, etwa mit Blick auf Kommunalwahlkämpfe, für die Parlamentarier als „Aushängeschild“ der lokalen Parteiorganisation dienen können. Sofern Wähler mit Migrationshintergrund dort räumlich konzentriert sind, kann es daher auch eine vielversprechende Wahlkampfstrategie sein, lokal verwurzelte Parlamentarier dazu anzuhalten, sich für die Interessen dieser Gruppen im nationalen Parlament einzusetzen. Mit anderen Worten: ein Zusammenhang zwischen einer lokalen Konzentration von Bürgern mit Migrationshintergrund und ihrer substantiellen Repräsentation durch individuelle Parlamentarier könnte auch Ergebnis eines dezentralisierten 
Kandidatennominierungsverfahrens sein. Wenn jedoch gesamtstaatlich organisierte Parteiakteure über zukünftige Kandidaturen entscheiden, dann sollten Parlamentarier weniger Anreize haben, lokal definierte Interessen $\mathrm{zu}$ vertreten, sondern vor allem eine Parteireputation auf gesamtstaatlicher Ebene pflegen.

Darüber hinaus können alle Parlamentarier als Repräsentationsagenten ihrer Fraktionsführungen aufgrund parlamentsinterner Organisationsstrukturen verstanden werden: ,virtually all legislators are subject to influence by at least one principal - their legislative party leadership“ (Carey, 2009, 133). ${ }^{13}$ Als Parteiakteure auf gesamtstaatlicher Ebene haben Fraktionsführungen vor allem das Interesse, die im nationalen Parteiprogramm definierten politischen Inhalte durchzusetzen und dadurch Wählern ein glaubwürdiges weil kohärentes Erscheinungsbild der Partei zu präsentieren, um den Stimmenanteil der Partei auf gesamtstaatlicher Ebene zu maximieren (Müller, 2000; Snyder \& Ting, 2002; Strøm \& Müller, 1999). Sowohl die vertikale als auch die horizontale Dimension der parlamentsinternen Organisationsstruktur (Strøm, 1998) geben den Fraktionsführungen Mittel, um ihre Parlamentarier dazu zu bewegen, im Sinne dieser kollektiven Ziele zu handeln. Die vertikale Dimension konstituiert sich aus hierarchisch organisierten Fraktionen, an deren Spitze die Fraktionsvorstände und an deren Ende die (Mehrheit der) sogenannten Hinterbänkler stehen (Saalfeld \& Strøm, 2014). Die Fraktionsspitze kann den Hinterbänklern bestimmte Privilegien einräumen, aber auch Sanktionsmittel einsetzen, um ein bestimmtes Verhalten zu belohnen oder zu bestrafen: Patronage und Beförderung zu bzw. Entlassung aus höheren Ämtern im Parlament, Mitgliedschaften in bzw. Abberufungen aus begehrten Ausschüssen, Zugang zu Reden in öffentlichkeitswirksamen Parlamentsdebatten, Medienauftritte, Büroräumlichkeiten und Personalausstattung und eine Reihe weiterer Vorzüge oder Bestrafungen (Bailer, 2017; Sieberer, 2006). Je mehr den Fraktionsführungen von diesen Maßnahmen zur Verfügung stehen, umso mehr Mittel halten sie auch in den Händen um Parlamentarier dazu zu bewegen, im Sinne kollektiver Parteiziele zu arbeiten.

\footnotetext{
${ }^{13}$ Dies ist eine die Realität vereinfachende Annahme. Laut Saalfeld und Strøm (2014) könnte das Verhältnis zwischen Parlamentariern und Parteien auch in dem Sinne betrachtet werden, dass Fraktionsführungen Agenten ihrer Parlamentarier als Prinzipale sind, weil letztere erstere in der Regel autorisieren. Die Annahme eines umgedrehten Prinzipal-Agenten Verhältnis kann jedoch gerechtfertigt werden, weil Parlamentarier ihre Fraktionsführungen mit der nötigen Autorität und Macht ausstatten, um einzelne Parlamentarier auf die kollektiven Positionen der Fraktion zu verpflichten. „[T]hese rights, the leaders' informational advantage over backbench members, their privileged media access, and their staffing resources may be strong enough to turn the agency relationship between caucus (as the principal) and the leader (as the agent) effectively on its head" (Saalfeld \& Strøm, 2014, 375)
} 
Die vertikale Dimension interagiert mit der horizontalen Dimension der Parlamentsorganisation, den Parlamentsausschüssen (Damgaard, 1995; Saalfeld \& Strøm, 2014; Strøm, 1998). Notwendigerweise müssen Fraktionseliten die inhaltlichen Ziele der Partei nach Politikfeldern differenzieren. Ausschüsse garantieren hierfür eine effiziente Arbeitsteilung zwischen den Parlamentariern der Fraktion und helfen so den Fraktionsführungen, die in parlamentarischen Demokratien fast immer das letzte Wort in der Festlegung der Ausschussmitgliedschaften haben (Damgaard, 1995, 313-315), kollektive politische Ziele zu verfolgen. In diesem Sinne kann eine ausgeprägte ausschussorientierte Arbeitsweise von Parlamenten (so genannte Arbeitsparlamente im Gegensatz zu Redeparlamenten) (Steffani, 1967) als ein Mittel der Fraktionsführungen betrachtet werden, die arbeitsteiligen Tätigkeiten ihrer Parlamentarier im Sinne der kollektiven Ziele der Partei zu koordinieren, wodurch Parlamentarier auch in ihren Ausschüssen im Interesse ihrer Fraktionen zu handeln haben (Cox \& McCubbins, 2007; Damgaard, 1995; Miller \& Stecker, 2008). Das Ausmaß, in dem die Ausschussmitgliedschaft das parlamentarische Verhalten auch außerhalb der Ausschüsse beeinflusst, kann daher als Indikator dafür betrachtet werden, inwieweit Parlamentarier die Erwartungen ihrer Fraktionen als politikfeldspezialisierte Agenten erfüllen. Sofern das nationale Parteiprogramm die Verbesserung der Lebenssituation von Bürgern mit Migrationshintergrund als inhaltliches Ziel definiert, und wenn dieser Themenkomplex auch in die Politikfeldspezialisierung von Parlamentariern fällt, sollten diese somit auch einen Anreiz haben, die Interessen benachteiligter Einwanderergruppen zu vertreten.

Nach der Erörterung dieser drei institutionellen Anreizstrukturen untersucht der dritte Aufsatz insbesondere die Frage, welche Prinzipale das parlamentarische Verhalten determinieren, wenn die beschriebenen institutionellen Strukturen Anreize generieren, den Forderungen unterschiedlicher Prinzipale Folge zu leisten. Diese Frage wird dabei jedoch vornehmlich als eine empirische betrachtet, die durch eine Untersuchung schriftlicher parlamentarischer Fragen im 17. Bundestag (2009-2013) bearbeitet wird. Genauer gesagt werden im dritten Aufsatz die beschriebenen institutionellen Einflussfaktoren auf die substantielle Repräsentation benachteiligter Einwanderergruppen anhand eines konkurrierenden Hypothesentests empirisch gegenüberstellt.

Der vierte Aufsatz dieser Dissertation „Immigration-related Speechmaking in a Partyconstrained Parliament: Evidence from the 'Refugee Crisis' of the 18th German Bundestag (2013-2017)“ geht in ähnlicher Weise vor und analysiert das migrationsspezifische 
Redeverhalten individueller Parlamentarier während der Flüchtlingskrise des 18. Bundestags. Mit einem konkurrierenden Hypothesentest wird vordergründig die Rolle der Fraktionsführung als „Gatekeeper“ im Sinne eines institutionellen Hemmnisses ins Zentrum des Aufsatzes gestellt. Ausgangspunkt ist zunächst die Annahme, dass Parlamentarier Redezeiten im Plenum zu migrations- und integrationspolitischen Themen anstreben, um individuelle Ziele zu verfolgen. Einerseits sollten Parlamentarier mit einem „sichtbaren“14 Migrationshintergrund aus intrinsischen Motiven $\mathrm{zu}$ diesen Themen das Wort ergreifen wollen (vgl. S. 3-4 in Aufsatz 4). Andererseits könnten sich Parlamentarier aber auch aus extrinsischen Motiven äußern wollen, wenn migrationsspezifische Themen eine hohe Relevanz für ihre Wähler haben. Vor allem unter den Bedingungen kandidatenzentrierter Wahlsystemregeln sollten sie einen individuellen Anreiz haben, sich in ihren Reden zu migrations- und integrationspolitischen Themen zu äußern, um ihren Wählern entsprechende Signale zu senden (vgl. S. 4-5 in Aufsatz 4).

Eine dritte theoretische Perspektive argumentiert jedoch, dass parlamentarische Reden individuellen Parlamentariern nicht notwendigerweise den Raum geben, ihre individuellen Ziele zu verfolgen, weil der Zugang zum Rednerpult von den Fraktionsführungen kontrolliert wird. Weil die Fraktionsführungen darüber entscheiden, wer sich zu welchen Themen am Rednerpult äußern darf, agieren individuelle Parlamentarier am Rednerpult im Wesentlichen auf Anweisung und mit dem Einverständnis ihrer Fraktionen (Cox, 2006; Proksch \& Slapin, 2015). Dadurch sollte die Auswahl von Rednern vor allem den kollektiven Zielen der Partei dienen, also der öffentlichen Präsentation einer einheitlichen parteipolitischen Position (policy), die dem Zweck dient, die Glaubwürdigkeit und Reputation des programmatischen Parteiprofils gegenüber Wählern zu gewährleisten, um die kollektiven Stimmenmaximierungsziele (votes) der Partei nicht zu gefährden (Bäck \& Debus, 2018; Proksch \& Slapin, 2015; Snyder \& Ting, 2002).

Einerseits erfordert diese Zielsetzung eine möglichst effiziente politikfeldspezifische Arbeitsteilung innerhalb der Fraktion, um die Repräsentation der Parteipositionen politikfeldübergreifend sicherzustellen. Um dies $\mathrm{zu}$ gewährleisten, berücksichtigen Fraktionsführungen die politikfeldspezifische Expertise von Parlamentariern bei der Verteilung von Redezeiten. Parlamentarier mit Migrationshintergrund könnten in diesem Sinne aufgrund ihrer Lebenserfahrungen von Fraktionsführungen etwa verstärkt als

\footnotetext{
${ }^{14}$ Die Sichtbarkeit des Migrationshintergrundes bezieht sich auf objektiv identifizierbare Merkmale des äußerlichen Erscheinungsbildes oder des Namens von Personen, die einen Migrationshintergrund suggerieren (siehe hierzu ausführlich S. 12-13 in Aufsatz 4).
} 
migrationspolitische Experten wahrgenommen werden (Nergiz, 2014, 254; Wüst \& Saalfeld, 2010, 331). Wenn ihr Migrationshintergrund darüber hinaus auch „sichtbar“ für Wähler ist, könnten Fraktionsführer diese Parlamentarier außerdem dafür nutzen, diese wahrgenommene Expertise im Plenum gegenüber Wählern zu signalisieren. Die Neigung von Parlamentariern mit Migrationshintergrund sich häufiger zu migrations- und integrationsspezifischen Themen im Plenum zu äußern, sollte nach dieser Lesart dann aber vor allem ein Resultat des Kalküls der Fraktionsführungen sein. Diese Erklärung konkurriert daher mit der ersten theoretischen Perspektive, nach der sich Parlamentarier häufiger $\mathrm{zu}$ entsprechenden Themen aus rein intrinsischen Motiven äußern. Um die Stichhaltigkeit dieser beiden Hypothesen zu prüfen, hebt der vierte Aufsatz vor allem zwei Bestimmungsfaktoren der migrationspolitischen Aufmerksamkeit der Reden von Parlamentariern mit Migrationshintergrund im Vergleich zu anderen Parlamentariern hervor.

Erstens sollte sich die durch die Fraktionsführungen zugewiesene politikfeldspezifische Spezialisierung in den Ausschussmitgliedschaften der Politiker reflektieren. Wie bereits erklärt, garantieren Ausschüsse eine effizientere Arbeitsteilung innerhalb der Fraktion. Spielt diese Arbeitsteilung auch eine Rolle bei der Rednerwahl der Fraktionsführungen, dann sollte sich der Zusammenhang zwischen dem Status als Politiker mit Migrationshintergrund und einer stärkeren Fokussierung auf migrationsspezifische Themen hauptsächlich durch ihre Mitgliedschaft in migrations- und integrationspolitikspezifischen Ausschüssen erklären lassen (z.B. die Ausschüsse für Inneres oder Soziales und Arbeit, und nicht etwa die Ausschüsse für Umwelt oder Verteidigung). Sollten hingegen intrinsische Motive den Zusammenhang zwischen Parlamentariern mit Migrationshintergrund und migrationspolitischen Reden erklären, dann sollte dieser auch unabhängig von den Ausschussmitgliedschaften der Parlamentarier existieren.

Zweitens kann vermutet werden, dass die Möglichkeiten einer effizienten Arbeitsteilung innerhalb der Fraktion von der Mitgliederstärke der Fraktion abhängen. Größere Fraktionen können eine differenziertere Arbeitsteilung gewährleisten als kleinere, weil mehr Abgeordnete zur Verfügung stehen, die auf eine gleich große Anzahl an Politikfeldern/ Ausschüssen verteilt werden können. Dadurch können Parlamentarier größerer Fraktionen ihre Arbeit in der Regel auf enger zugeschnittene Themenfelder spezialisieren. Der Mangel an Personal in kleineren Fraktionen macht es hingegen notwendig, dass Parlamentarier ihre Expertise auf mehrere Themenfelder ausweiten müssen. Dieser Zusammenhang wird auch in der Anzahl der Ausschussmitgliedschaften von Parlamentariern deutlich, die in größeren 
Fraktionen tendenziell in weniger Ausschüssen Mitglieder sind (Ismayr, 1992, 189-191, 2012, 168). Dadurch sind die Politikfeldspezialisierungen der Parlamentarier in größeren Fraktionen auch eindeutiger voneinander abgrenzbar, während die der Parlamentarier in kleineren Fraktionen diverser und dadurch schlechter voneinander abgrenzbar sind. Sofern die fraktionsinterne Arbeitsteilung auch eine Rolle für die Auswahl von Rednern spielt, sollte sich dieser Zusammenhang auch auf die themenfeldspezifischen Redebeiträge der Parlamentarier übertragen lassen. Parlamentarier in größeren Fraktionen sollten dadurch insgesamt über weniger Themen sprechen, während Parlamentarier in kleineren Fraktionen insgesamt über mehrere Themen sprechen sollten. Dadurch sollten auch die Redebeiträge von Parlamentariern in größeren Fraktionen in ihrer Zusammenschau tendenziell spezialisierter sein als die der Parlamentarier in kleineren Fraktionen. Übertragen auf die thematische Schwerpunktsetzung der Reden von Politikern mit Migrationshintergrund auf Migrations- und Integrationsthemen hieße dies, dass sich diese auch stärker von den Schwerpunkten ihrer Parteikollegen in größeren als in kleineren Fraktionen unterscheiden lassen sollte. Wenn hingegen intrinsische Motivationen dieser Schwerpunktsetzung unterliegen, sollten die Bedingungen für eine effiziente Arbeitsteilung innerhalb der Fraktionen keine gleichermaßen starke intervenierende Wirkung auf die unterschiedlichen Themenaufmerksamkeiten von Parlamentariern mit „sichtbarem“ Migrationshintergrund haben.

Darüber hinaus sollten Fraktionsführungen ebenso einen Anreiz haben, die Rednerauswahl von den individuellen Wiederwahlkontexten ihrer Parlamentarier (unabhängig vom Migrationshintergrund) abhängig zu machen. Dies trägt vor allem dem kollektiven Ziel Rechnung, die Stimmen- und Sitzanteile in zukünftigen Wahlen insgesamt zu maximieren. Fraktionsführungen sollte daran gelegen sein, Reden aus den eigenen Reihen zu verhindern, welche der Parteilinie und dadurch der Präsentation einer kohärenten Parteiposition widersprechen. Laut Proksch und Slapin (2015) sei das Ausmaß, in dem Parteieliten gegenüber Wählern signalisierte parteipolitische Positionen kohärent und einheitlich halten wollen, eine Funktion der Kandidatenzentriertheit des Wahlsystems. In parteizentrierten Wahlsystemen würde hauptsächlich die Reputation der Partei auf nationaler Ebene den aggregierten Wahlerfolg der Partei determinieren. In kandidatenzentrierten Wahlsystemen würde der kollektive Wahlerfolg hingegen wesentlich durch die Fähigkeit individueller Kandidaten beeinflusst, eine persönliche Reputation mit lokal definierten Wählergruppen zu pflegen. Fraktionsführungen hätten unter diesen Bedingungen daher geringere Anreize, ein kohärentes und einheitliches Parteiprofil zu pflegen, so dass sie von der Parteilinie 
abweichendes Redeverhalten weniger kritisch sehen würden. Die Autoren klassifizieren ${ }^{15}$ Mehrheitswahlsysteme in Einpersonenwahlkreisen als kandidatenzentrierte Systeme, welche besonders starke Anreize generieren würden, Wähler auf der Grundlage persönlicher Reputationen zu mobilisieren. Verhältniswahlsysteme mit geschlossenen Kandidatenlisten seien hingegen parteizentriert, das heißt mit besonders schwachen Anreizen für personenbezogene Reputationspflege assoziiert. Verhältniswahlsystemen mit offenen/flexiblen Listen würden hingegen eine mittlere Position zwischen diesen beiden Extremen einnehmen (Proksch \& Slapin, 2015, 44-49).

In kompensatorischen personalisierten Verhältniswahlsystemen (mixed-member proportional systems) bestimmen die Stimmenanteile im Listenwahlsegment die Sitzanteile der Parteien, so dass die Reputation der Partei den aggregierten Wahlerfolg der Partei maßgeblich bestimmen sollte. Parteiführungen sollten unter diesen Bedingungen also einen starken Anreiz haben, Wählern eine kohärente parteipolitische Position zu präsentieren. Andererseits sollten Wahlkreisabgeordnete jedoch eigene elektorale Anreize haben, eine persönliche Reputation mit ihren Wählern zu pflegen, die mit der Abgrenzung der politischen Position des Abgeordneten von der kollektiven Parteilinie einhergehen kann (Bäck \& Debus, 2018, 74; Proksch \& Slapin, 2015, 45; siehe auch Sieberer, 2010, 485). Aus der Motivation heraus die Einheitlichkeit der kollektiven parteipolitischen Position gegenüber abweichendem

\footnotetext{
${ }^{15}$ Die von Proksch und Slapin vorgeschlagene Reihenfolge ist jedoch nicht unumstritten. Uneinigkeit wird häufig bezüglich der Einordnung von Mehrheitswahlsystemen mit Einpersonenwahlkreisen geäußert. Laut Carey und Shugart seien diese gleichzusetzen mit geschlossenen Parteilisten mit nur einem Kandidaten, so dass die Wahlkreisgröße die einzige Größe darstelle, durch die Mehrheitswahlsysteme in Einpersonenwahlkreisen von Verhältniswahlsystemen mit geschlossenen Parteilisten abzugrenzen seien (vgl. Carey und Shugart 1995, 422). Carey und Shugart assoziieren in der Konsequenz Wahlsysteme mit offenen/flexiblen Listen mit stärkeren Anreizen für die Pflege einer persönlichen Reputation als Mehrheitswahlsystemen in Einpersonenwahlkreisen. Wallack und Coautoren argumentieren jedoch gegen diese Behandlung und messen Einpersonenwahlkreisen wesentlich stärkere Anreize zur personenbezogenen Reputationspflege bei. Erstens würden Parteien in Mehrheitswahlsystemen relativ stärker davor zurückschrecken, populären Parlamentariern die erneute Aufstellung als Kandidat zu verweigern:,,[c]andidates' popularity with voters in such districts is likely to be more important in gaining access to a list of one than a list of several“ (Wallack et al., 2003, 137). Zweitens seien die Anreize eine gemeinsame Parteireputation zu pflegen geringer als von Carey und Shugart vermutet, weil Kandidaten in Mehrheitswahlsystemen nicht von der elektoralen Performanz anderer Kandidaten derselben Partei profitieren würden: „[candidates] do not receive additional electoral support if other candidates from their party are successful in other districts" (ebd., 138). In der von Wallack und Koautoren vorgeschlagenen alternativen Kodierung von Wahlsystemen werden Mehrheitswahlsysteme in Einpersonenwahlkreisen daher mit stärkeren Anreizen zur Pflege persönlicher Reputationen assoziiert als Systeme mit offenen/flexiblen Listen. Ähnlich argumentieren auch Norris (2004, 230-232) sowie Proksch und Slapin (2015, 44-49), dass nur bestimmte Wahlsysteme, die innerparteilichen Wettbewerb in Mehrpersonenwahlkreisen zulassen, eine ähnlich starke Kandidatenzentrierung wie Mehrheitswahlsysteme in Einpersonenwahlkreisen generieren würden. Die letzten beiden Aufsätze dieser Dissertation werden von dieser Kontroverse jedoch nur am Rande tangiert. Unabhängig davon welches Kodierschema zur Anwendung kommt, sollten Mehrheitswahlen in Einpersonenwahlkreisen immer relativ stärkere Anreize als geschlossenen Parteilisten in Mehrpersonenwahlkreisen generieren, eine persönliche Reputation zu pflegen.
} 
Individualverhalten zu schützen, sollten Fraktionen dann Wahlkreisabgeordneten insgesamt weniger Redezeit zugestehen als Listenabgeordneten (Bäck \& Debus, 2018, 74; Proksch \& Slapin, 2015, 45).

Eine wichtige intervenierende Variable für diese Anreize in Einpersonenwahlkreisen ist jedoch der individuelle Stimmenvorsprung der Parlamentarier in ihren Wahlkreisen (André et al., 2015; Zittel \& Gschwend, 2008). Diese Überlegung wird in dem vierten Aufsatz aufgegriffen und in die Erklärung migrationsspezifischen Redeverhaltens von Parlamentariern integriert. Sofern Wahlkreisabgeordnete aus Wahlkreisen kommen, die sogenannte „Parteihochburgen“ sind, also wenn sie über einen komfortablen Stimmenvorsprung verfügen, sollten mögliche Stimmenverluste weniger schwer wiegen als für Parlamentarier, die ihre Wahlkreise mit sehr knappen Stimmenvorsprüngen gewonnen haben. Dadurch hätten vor allem Abgeordnete aus knappen Wahlkreisen stärkere Anreize, eine persönliche Reputation in ihren Wahlkreisen $\mathrm{zu}$ pflegen. Unter diesen Bedingungen sollten Fraktionsführungen daher eine gesunde Skepsis vor allem gegenüber Redebeiträgen von Wahlkreisabgeordneten an den Tag legen, die ihre Wahlkreise nur mit einem knappen Stimmenvorsprung gewonnen haben. Weil die Gefahr besteht, dass dieser Parlamentariertypus Redezeit nutzen möchte, um politische Signale an Wahlkreise zu senden, welche nicht notwendigerweise im Einklang mit der kollektiven Parteilinie sein müssen, haben Fraktionsvorstände daher den reziproken Anreiz diese Parlamentarier weniger zu Wort kommen zu lassen.

Dieser Anreiz sollte besonders ausgeprägt sein, wenn hochgradig saliente Politikfelder debattiert werden, die von hoher Wichtigkeit in der Problemwahrnehmung von Wählern sind. Als Politikfeld, das seit geraumer Zeit ein hohe Wichtigkeit in der Problemwahrnehmung der Wähler hat, sollte etwaiges Verhalten der Parlamentarier im Themenbereich der Migrationsund Integrationspolitik daher von den Fraktionsführungen als besonders kritisch gesehen werden. Wenn die „Gatekeeper“-Funktion der Fraktionsführungen als institutionelles Hemmnis das integrations- und migrationspolitische Redeverhalten also maßgeblich strukturiert, im Gegensatz zu den individuellen elektoralen Anreize der Parlamentarier, dann sollten Wahlkreisabgeordneten in der Tendenz auch umso weniger über diese Themen sprechen, je größer ihr individueller elektoraler Anreiz ist, eine persönliche Reputation im Wahlkreis zu pflegen. 


\subsection{Fallauswahl, Daten und Methoden}

Sowohl Aufsatz 3 als auch Aufsatz 4 präsentieren empirische Untersuchungen des parlamentarischen Verhaltens von Mitgliedern des Bundestags. Abgesehen von der gesellschaftlichen Relevanz Deutschlands für die Erforschung von Repräsentationsmechanismen in Einwanderungsgesellschaften, wie bereits unter Abschnitt 3.3 ausgeführt, bietet sich Deutschland aus mehreren Gründen für eine empirische Überprüfung der konkurrierenden Hypothese der beiden Aufsätze an.

Mit Blick auf die Forschungsfrage des dritten Aufsatzes dieser Dissertation ist eine Fallstudie des Bundestags geeignet, weil die Institutionenkombination von Wahlsystem, Kandidatenauswahlverfahren und parlamentsinterner Organisation im Sinne der theoretischen Überlegungen entgegenlaufende Verhaltensanreize für Parlamentarier generieren sollte, die Interessen unterschiedlicher Prinzipale zu repräsentieren. Gleichzeitig können durch die Analyse eines Landes Besonderheiten des länderspezifische Kontexts konstant gehalten werden, so dass die institutionellen Einflussfaktoren analytisch isoliert und unterscheidbar gemacht werden können (Moser \& Scheiner, 2012, 46). Wie bereits unter Abschnitt 3.3 erklärt, werden Parlamentarier entweder in lokalen Wahlkreisen oder über geschlossene Landeslisten gewählt und sollten somit nach dem Wahlsystemargument abweichende Anreize haben, entweder lokale Wählergruppen oder zentralisierte Parteiakteure stärker zu repräsentieren. Gleichzeitig sorgt jedoch die Doppelkandidatur-Praxis in der Kandidatenauswahl (vgl. Abschnitt 3.3) dafür, dass sowohl Wahlkreis- als auch Listenabgeordnete eine erneute Wahlkreiskandidatur sicherstellen müssen, um wiedergewählt zu werden. Das Kandidatenauswahlverfahren hat daher tatsächlich für alle Parlamentarier einen dezentralen Charakter. Somit können konkurrierende Hypothesen, welche die Effekte der drei genannten Anreizstrukturen einander gegenüberstellen, am deutschen Fall überprüft werden. Allgemein wird angenommen, dass Wahlkreisinteressen substantiell repräsentiert werden, wenn Parlamentarier umso mehr parlamentarische Fragen über die gesellschaftliche Benachteiligung von Einwanderergruppen stellen, je größer der Anteil von Migranten in ihrem Wahlkreis ist. Das Wahlsystemargument sollte dann Unterstützung erfahren, wenn dieser Zusammenhang nur für Parlamentarier gilt, die in einem Wahlkreis gewählt wurden. Demgegenüber sollte das Argument der Kandidatenaufstellung stärkere Unterstützung erfahren, wenn dieser Zusammenhang nicht nur für Parlamentarier mit Wahlkreismandaten 
gilt, sondern unabhängig vom Wahlmodus für alle Parlamentarier, die in einem Wahlkreis aufgestellt wurden.

Außerdem generiert die Organisationsstruktur des Bundestags starke Anreize, den kollektiven Interessen der Fraktion zu dienen. Der Deutsche Bundestag kann als parteidominiertes Arbeitsparlament bezeichnet werden, das hierarchisch organisierte Parteifraktionen mit einem nach Politikfeldern ausdifferenzierten Ausschusssystem kombiniert, und dadurch eine von den Fraktionen dominierte politikfelddifferenzierte Arbeitsteilung zwischen den Parlamentariern nach sich zieht (Ismayr, 2012, 162; Miller \& Stecker, 2008). Die Dominanz der Fraktionen und der Ausschussarbeit zeigt sich insbesondere anhand der folgenden vier Merkmale. Erstens findet der Großteil der Arbeit von Parlamentariern in Ausschüssen statt (Ismayr, 2012). Zweitens haben die Fraktionsführungen, welche aus den Fraktionsvorsitzenden, stellvertretenden Fraktionsvorsitzenden, parlamentarischen Geschäftsführern und Arbeitsgruppenvorsitzenden bestehen (Saalfeld, 2000, 29), das letzte Wort bei der Verteilung von Ausschussmitgliedschaften (Damgaard, 1995). Drittens finden Ausschusssitzungen mit wenigen Ausnahmen unter Ausschluss der Öffentlichkeit statt und sind somit nur für Fraktionsführungen als Prinzipale überwachbar (Ismayr, 2012; Miller \& Stecker, 2008). Viertens werden die Ausschüsse durch fraktionsinterne Arbeitsgruppen gespiegelt, in denen in wöchentlichen Sitzungen unter Leitung der Vorsitzenden der Arbeitsgruppen die Positionen der Partei in den Ausschüssen vorbereitet werden (Ismayr, 2012; Miller \& Stecker, 2008).

Können individuelle Parlamentarier während der Beratungen in den Arbeitsgruppen die kollektive Position der Fraktion noch mitgestalten, sind sie im Anschluss daran an diese gebunden: „After a decision has been reached in the parliamentary party group, individual members are expected to respect the majortiy decision and toe the party line" (Saalfeld, 2000, 25). Als Politikfeldexperten sind einzelne Parlamentarier in ihren Ausschüssen daher gehalten, die kollektiven inhaltlichen Schwerpunkte und Positionen der Fraktion zu repräsentieren, wenn sie keine negativen Reaktionen der Fraktionsführung riskieren wollen, die von subtilem sozialem Druck über den Verlust der Ausschussmitgliedschaft bis hin zur Androhung eines Karriereabstiegs oder der Verweigerung eines Karriereaufstiegs innerhalb der Fraktionshierarchie reichen (Bailer, 2017; Ismayr, 2012; Miller \& Stecker, 2008).

Die parlamentsinterne Anreizstruktur kann somit Verhaltensanreizen gegenüber gestellt werden, die sich aus dem Wahlmodus (Wahlkreis vs. Liste) der Abgeordneten ergeben. Sofern sich die Thematisierung der gesellschaftlichen Benachteiligung vornehmlich aus den 
Erwartungen der Fraktionsführungen ergeben, dann sollten zwei Faktoren eine strukturierende Wirkung auf das Frageverhalten aller Parlamentarier gleichermaßen haben: die Tätigkeit in migrationsnahen Ausschüssen und die Mitgliedschaft in Parteien, die in ihren Parteiprogrammen die Verbesserung der Lebensverhältnisse von Bürgern mit Migrationshintergrund fordern. Im Gegensatz dazu würde das Wahlsystemargument postulieren, dass diese Faktoren als Indikatoren der Erwartungen zentralisierter Parteiakteure hauptsächlich für das parlamentarische Verhalten von Abgeordneten mit Listenmandat, und weniger für Abgeordnete mit Wahlkreismandat, eine Rolle spielen sollten.

Mit Blick auf den vierten Aufsatz dieser Dissertation bietet sich eine Fallstudie des Deutschen Bundestags für die Analyse der Bedeutung institutioneller Hemmnisse für das Redeverhalten von Parlamentariern an. Hinsichtlich der Möglichkeit parlamentarische Reden im Bundestag zu halten, ist es plausibel, die Rolle der Fraktionen als die eines „Gatekeepers“ zu sehen. Erstens entscheiden Repräsentanten der Fraktionsführungen die Themen der Plenumsdebatten sowie die Redezeiten der Fraktionen im Ältestenrat (Schüttemeyer, 1994, 37). Zweitens können sich Parlamentarier nicht etwa spontan in Redelisten eintragen, sondern werden in den fraktionsinternen Arbeitsgruppen dafür eingeteilt (Ismayr, 2012, 305). Vor allem der 18. Bundestag (2013-17) bietet sich für eine Untersuchung der Rolle der Fraktionen als Gatekeeper für das migrations- und integrationsrelevante Redeverhalten individueller Parlamentarier an, war diese Legislaturperiode schließlich von einer besonders hohen Salienz des Migrations- und Integrationsthemas in der öffentlichen Wahrnehmung geprägt. Diese speiste sich vor allem aus dem ansteigenden Zustrom von Flüchtlingen aus den Krisengebieten des Nahen und Mittleren Ostens, der 2015 in der sogenannten „Flüchtlingskrise“ seinen Höhepunkt fand und den Aufstieg der AfD (Alternative für Deutschland) als ernstzunehmende, den Parteienwettbewerb beeinflussende rechtspopulistische Partei einläutete. Weil die etablierten Parteien, allen voran die der Großen Koalition, dadurch auf migrationspolitischem Terrain zunehmend in die Defensive gerieten und die eigenen Stimmenmaximierungsziele gefährdet sahen, ist davon auszugehen, dass die kollektiven Ziele der Parteien für die Auswahl von Rednern in migrationspolitischen Debatten eine große Rolle gespielt haben sollten. Daher ist der Kontext dieser Wahlperiode besonders geeignet für eine empirische Überprüfung der konkurrierenden Hypothesen des vierten Aufsatzes.

Die Wahl der Indikatoren des substantiellen Repräsentationsverhaltens individueller Parlamentarier, das heißt schriftliche parlamentarische Fragen im Fall des dritten sowie 
parlamentarische Reden im Fall des vierten Aufsatzes, kann wie folgt gerechtfertigt werden. Zunächst einmal ist es wichtig die Bevorzugung dieser Indikatoren gegenüber Abstimmungsverhalten als Indikator substantieller Repräsentation zu begründen. Schließlich gelten Abstimmungen als ultimative Akte des parlamentarischen Prozesses, die Abstimmungsgegenständen Gesetzeskraft geben können (Bowler et al., 1999; Sieberer, 2006). Aus mehreren Gründen bietet sich eine Untersuchung legislativer Abstimmungen im Kontext dieser Dissertation jedoch nicht an.

Abstimmungen im Bundestag unterliegen im Allgemeinen der Fraktionsdisziplin (Saalfeld, 1995), repräsentieren hauptsächlich die kollektive Mehrheitsmeinungen im Anschluss an die innerfraktionelle Entscheidungsfindung (Wessels, 1993), bestehen nur aus drei Verhaltensoptionen (Zustimmung, Ablehnung oder Enthaltung) (Geese et al., im Erscheinen) und werden nur in einer Minderheit der Fälle namentlich dokumentiert (Bergmann et al., 2016; Carrubba et al., 2006). Aus diesen Gründen bleiben die individuellen Motive des Repräsentationsverhaltens in Analysen von Abstimmungen weitgehend im Verborgenen. Parlamentarier verfügen jedoch über eine Reihe alternativer innerparlamentarischer Instrumente, mit denen sie Themenschwerpunkte und Positionen mit anderen Akteuren kommunizieren und in parlamentarischen Protokollen in Textform dokumentieren lassen können: Plenumsreden, Berichterstattungen zu Ausschusstätigkeiten, parlamentarische Anfragen/Interpellationen, sowie mündliche und schriftliche parlamentarische Fragen (Keh, 2015). Umfassende und zeitlich zurückreichende Dokumentationen dieser Tätigkeiten lassen sich aus den elektronischen Archiven des Bundestags extrahieren und stehen so Politikwissenschaftlern zur wissenschaftlichen Analyse zur Verfügung. Darüber hinaus garantiert die Dokumentation in Textform einen hohen inhaltlichen Informationsgehalt, der durch den Einsatz inhaltsanalytischer Methoden erfasst werden kann.

Laut Keh sind die genannten Parlamentsaktivitäten nach ihrem Zentralisierungsgrad zu unterscheiden. Dezentralisierte Parlamentsaktivitäten liegen stärker im Ermessen einzelner Parlamentarier, während zentralisierte Parlamentsaktivitäten wesentlich von den Fraktionsführungen gesteuert bzw. von diesen an einzelne Parlamentarier delegiert werden (Keh, 2015). Um Einblicke in die persönlichen Repräsentationsentscheidungen von Parlamentariern als Reaktion auf institutionelle Verhaltensanreize zu bekommen, eignen sich daher vor allem dezentralisierte Parlamentsaktivitäten. Schriftliche parlamentarische Fragen sind im deutschen Bundestag die am stärksten dezentralisierten Instrumente, die Parlamentariern zur Verfügung stehen, um ihre Themenschwerpunkte und Positionen zu 
kommunizieren (Keh, 2015, 1097). Individuelle Parlamentarier können schriftliche Fragen relativ frei nutzen, um die Aufmerksamkeit der Regierung und anderer Akteure auf selbstdefinierte Problemlagen zu lenken, um Informationen über gegenwärtiges oder beabsichtigtes Regierungshandeln einzuholen, um die eigene Ausschussarbeit mit Informationen zu unterfüttern, oder um die Antworten der Regierung auf die eigens gestellten Fragen im Internet oder in traditionellen Medien zu veröffentlichen (Bailer, 2011; Martin, 2011; Rozenberg \& Martin, 2011; Russo \& Wiberg, 2010; Siefken, 2010; Wiberg \& Koura, 1994).

Die Rolle der Fraktionen als Gatekeeper im Sinne einer institutionellen Hemmnissstruktur spielt hingegen vor allem in solchen Parlamentsaktivitäten eine Rolle, die stärker zentralisiert sind. Diese Aktivitäten bieten den Parlamentariern geringere Möglichkeiten, sich den politischen Themen $\mathrm{zu}$ widmen, die sie selbst für wichtig erachten, weil inhaltliche Schwerpunkte und Positionen von kollektiven Fraktionsentscheidungen in der Regel vorgegeben werden (Cox, 2006; Döring, 1995). Laut Keh ist die Plenumsrede das mit Abstand am stärksten zentralisierte der genannten Instrumente im deutschen Bundestag (Keh, 2015, 1097) und sollte daher auch als Untersuchungsgegenstand des vierten Aufsatzes am besten geeignet sein.

Da beide Aufsätze textbasierte Indikatoren parlamentarischen Verhaltens analysieren, verwenden sie auch textanalytische Methoden zur Messung migrations- und integrationspolitischer Inhalte. Aufsatz 3 verwendet dafür eine Datenbasis aller 20.130 schriftlicher parlamentarischer Fragen, die im 17. Bundestag (2009-13) an die Regierung gestellt und vom Koautor des Papiers, Carsten Schwemmer, im Rahmen des PATHWAYS Projekt (siehe S. 31 in diesem Rahmenpapier) aus den offiziellen Onlinearchiven des Bundestags (Bundestag, 2019) extrahiert wurden. Da der dritte Aufsatz die substantielle Repräsentation benachteiligter Einwanderergruppen untersucht, wurden schriftliche Fragen als integrationsrelevant definiert und gemessen, wenn sie politische, ökonomische und/oder soziale Benachteiligungen von Bürgern mit Migrationshintergrund thematisieren. Zum Zweck der Identifizierung dieser Fragen wurde ein mehrstufiges Kodierschema entwickelt, das ein computergestütztes automatisiertes Dictionary-Verfahren mit einem qualitativen Handkodierungsverfahren kombiniert (siehe hierzu S. 10-11 in Aufsatz 3, sowie S. 2-6 im Anhang von Aufsatz 3). Auf dieser Grundlage konnte die abhängige Variable als Anzahl der integrationsrelevanten schriftlichen Fragen je Parlamentarier gemessen werden. Fast alle unabhängigen Variablen wurden im Rahmen des PATHWAYS Projekts erhoben: der 
Ausländeranteil im Wahlkreis, der Wahlmodus der Parlamentarier (Wahlkreis vs. Liste) und der migrations- bzw. integrationspolitische Bezug ihrer Ausschussmitgliedschaften. Informationen über die Wichtigkeit der Integrationspolitik in den Parteiprogrammen stammen aus den Daten des Comparative Manifesto Projects (Volkens et al., 2015). Für die quantitative Überprüfung der Hypothese wurde ein sogenanntes zero-inflated negative binomial regression model verwendet (Cameron \& Trivedi, 2013, 111-76). Dieses Regressionsmodell ist für die Analyse von Zählvariablen geeignet und erlaubt zudem mit zwei wichtigen Einschränkungen umzugehen, die sich aus der Verteilung der abhängigen Variable ergeben: der hohen Anzahl an Parlamentariern, die keine integrationsrelevanten Fragen stellen, und der Neigung von Oppositionspolitikern mehr schriftliche Fragen als Politiker der Regierungsfraktionen zu stellen (siehe dazu ausführlich S. 12-13 in Aufsatz 3).

Der vierte Aufsatz dieser Dissertation basiert auf einer Analyse von über 10.000 Redebeiträgen individueller Parlamentarier im 18. Deutschen Bundestag (2013-2017). Die Daten sind über die Webseite www.offenesparlament.de, die vom gemeinnützigen Verein Open Knowledge Foundation Deutschland e.V. betrieben wird, frei verfügbar. Um migrationsrelevante Inhalte in parlamentarischen Reden zu messen, wird ein Structural Topic Model (STM) genutzt (M.E. Roberts et al., 2014). STM stellt ein vollautomatisiertes computergestütztes Verfahren der quantitativen Textanalyse dar (Grimmer \& Stewart, 2013). Topic Models können als statistische Clusteralgorithmen beschrieben werden, die auf der Grundlage gemeinsam auftretender Wörter in den Texten eines Textkorpus (Kookkurrenzen) sowohl einzelne Texte als auch den Gesamtkorpus in mehrere Themenkategorien einordnen (siehe S. 10-11 in Aufsatz 4, sowie den Anhang des vierten Aufsatzes für weitergehende Informationen und Validierungen der Messung). Die so in den Textdaten gemessenen Themenschwerpunkte der Reden wurden anschließend mit einem Datensatz biographischer, partei- und wahlkreisbezogener Informationen der Parlamentarier des 18. Bundestags verknüpft, welcher im Anschluss an das PATHWAYS Projekt am Lehrstuhl für Vergleichende Politikwissenschaft der Otto-Friedrich-Universität Bamberg (Prof. Dr. Thomas Saalfeld) erhoben wurde, um die Datengrundlage des Projekts zeitlich fortzuführen. ${ }^{16}$ Die wichtigsten unabhängigen Variablen aus diesem Datensatz umfassen dabei die Unterscheidung von Parlamentariern mit einem „sichtbaren“ Migrationshintergrund, des migrations- bzw. integrationspolitischen Bezugs der Ausschussmitgliedschaft, die Fraktionszugehörigkeit, dem Wahlmodus (Wahlkreis vs. Liste) und die Knappheit des

\footnotetext{
${ }^{16}$ Der Erhebungszeitraum des PATHWAYS Projekts endete 2013.
} 
Wahlkreisergebnisses in der Bundestagswahl 2013 (siehe hierzu ausführlich S. 11-13 in Aufsatz 4). Um die konkurrierenden Hypothesen quantitativ zu überprüfen, wird ein fractional response logit model genutzt, das für die Analyse prozentualer Anteile als abhängige Variable (hier: Anteil migrationsrelevanter Inhalte der Reden) geeignet ist (Papke \& Wooldridge, 1996).

Durch die Verwendung sogenannter „Themenmodelle“ (topic models) im vierten Aufsatz und die Verarbeitung großer Textmengen zur Messung migrations- und integrationspolitischer Inhalte im dokumentierten Verhalten individueller Parlamentarier in beiden Aufsätzen, leisten die Beiträge einen innovativen methodischen Beitrag zur migrationspolitischen Repräsentationsforschung. Obwohl politische Akteure im Zeitalter des Internets immer mehr öffentlich zugängliche und extrahierbare Spuren ihres Verhaltens hinterlassen, werden Politikwissenschaftlicher dadurch jedoch vor die Aufgabe gestellt, die schiere Menge dieser Daten nicht nur zu sammeln, sondern auch in eine geordnete Struktur $\mathrm{zu}$ bringen und empirisch zu analysieren (Grimmer \& Stewart, 2013). Die methodisch-analytischen Ansätze beider Aufsätze vollbringen diese Leistung für tausende von textbasierten Dokumenten (über 20.000 parlamentarische Fragen und über 10.000 parlamentarische Reden) und zeigen wie migrations- und integrationspolitische Inhalte durch den Einsatz moderner Methoden der quantitativen Textanalyse empirisch gemessen und analysiert werden können.

\subsection{Empirische Befunde}

In ihrer Zusammenschau können beide Aufsätze zeigen, dass mit dem gewählten PrinzipalAgent-Ansatz ein Erklärungsbeitrag zum migrations- und integrationspolitischen Verhalten von Parlamentariern geleistet werden kann.

So zeigen die empirischen Befunde des dritten Aufsatzes, dass die Erwartungen der Prinzipale das Verhalten der Parlamentarier hinsichtlich der substantiellen Repräsentation benachteiligter Einwanderergruppen weitreichend strukturieren. Die Anzahl integrationsrelevanter schriftlicher parlamentarischer Fragen, die Parlamentarier an die Regierung stellen, wird einerseits vom lokalen Anteil der Wähler mit Migrationshintergrund in den Wahlkreisen beeinflusst. Andererseits wird dieses Verhalten auch maßgeblich beeinflusst von einer Interaktion zwischen der Mitgliedschaft der Parlamentarier in migrations- und integrationsnahen Ausschüssen und ihrer Zugehörigkeit zu Parteien links der 
Mitte, welche die gesellschaftliche Besserstellung von Bürgern mit Migrationshintergrund in den Parteiprogrammen tendenziell stärker hervorheben. Dies erhärtet die These, dass sowohl lokal als auch national definierte Interessen das Repräsentationsverhalten deutscher Parlamentarier systematisch beeinflussen. Der gewählte analytische Ansatz, der auf das Verhalten aller Parlamentarier unabhängig vom Vorliegen eines Migrationshintergrundes ausgerichtet ist, kann somit einen Beitrag leisten, unser Wissen der substantiellen Repräsentation benachteiligter Einwanderergruppen zu verbessern.

Mit Blick auf die konkurrierenden Hypothesen besteht ein zentrales Ergebnis darin, dass die Responsivität der Parlamentarier gegenüber Wahlkreisen mit einem hohen Anteil an Wählern mit Migrationshintergrund auf der einen Seite und gegenüber kollektiven Parteiinteressen auf der anderen Seite nicht das Ergebnis unterschiedlicher Wahlmodi (Wahlkreis vs. Liste) ist. Stattdessen erhärten die Befunde, dass der lokale Repräsentationsfokus Resultat einer insgesamt dezentralisierten Kandidatennominierungspraxis ist, während der nationale Repräsentationsfokus Ergebnis einer parlamentsinternen Organisationsstruktur ist, in der die Dominanz der Fraktionen Verhaltensanreize generiert, kollektive Parteiinteressen zu repräsentieren.

Diese Befunde ergänzt der vierte Aufsatz durch eine empirische Analyse der migrationsspezifischen Aufmerksamkeit der Plenumsreden individueller Parlamentarier. Die Befunde zeigen, dass der Einfluss eines sichtbaren Migrationshintergrunds auf Seiten der Parlamentarier abhängig ist von der Ausschussmitgliedschaft und von der Größe der Parlamentsfraktion. Darüber hinaus scheint eine zunehmende Knappheit ${ }^{17}$ des Wahlergebnisses im Wahlkreis damit einherzugehen, dass Wahlkreisabgeordnete weniger über migrationsrelevante Themen in ihren Reden sprechen. Diese Befunde unterstützen daher die These, dass vor allem die kollektiven Motive der Fraktionen die Aufmerksamkeit der Reden individueller Parlamentarier auf Themen der Migrationspolitik beeinflussen. In diesem Lichte ist die Erklärungskraft individueller Repräsentationsmotive einzuschränken. Dieser Befund leistet einen wichtigen Forschungsbeitrag, weil dadurch zukünftige Forschungsunternehmungen dahingehend diszipliniert werden sollten, die kollektiven Ziele der Fraktionen in der Analyse des Verhaltens individueller Parlamentarier zu berücksichtigen, vor allem wenn nach Keh (2015) zentralisierte Parlamentsaktivitäten untersucht werden.

\footnotetext{
${ }^{17}$ Es ist allerdings darauf hinzuweisen, dass dieser Zusammenhang hauptsächlich innerhalb der CDU/CSU Fraktion zustande kommt. Die CDU/CSU gewann in der Bundestagswahl 2013 79\% aller Direktmandate. Darüber hinaus ist die Verteilung der höheren Stimmenvorsprünge stark Richtung CDU/CSU geneigt. Die Verteilung dieser Variablen in den anderen Parteien lässt hingegen nicht genügend Variation zu, um den Zusammenhang parteispezifisch zu überprüfen.
} 
Die Befunde beider Aufsätze eröffnen außerdem neue Wege für zukünftige Forschungsvorhaben. Einerseits gibt es aufgrund der Fokussierung der beiden Studien auf jeweils eine Legislaturperiode in einem Land einen Bedarf sowohl an Nachfolgestudien in anderen Ländern als auch an ländervergleichenden Studien, um die Generalisierbarkeit der Befunde zu überprüfen. Andererseits ergeben sich aber auch andere drängende Fragen, mit denen sich zukünftige Untersuchungen auseinandersetzen sollten.

Nachfolgeuntersuchungen könnten an den Befund des dritten Aufsatzes anschließen, der zeigt, dass die strukturierende Wirkung von Wahlsystemregeln durch andere institutionelle Einflussfaktoren konterkariert werden können. Der Einfluss von Wahlsystemregeln könnte etwa anderen als den im dritten Aufsatz untersuchten Institutionen gegenüberstellt werden, etwa bezogen auf den Einfluss von Lobbygruppen (Giger \& Klüver, 2015) oder bezogen auf das in vielen Ländern bestehende Erfordernis für Parlamentarier lokale und nationale Ämter und Mandate zu akkumulieren (Fernandes et al., 2018; Russo, 2011; Tavits, 2011). Ähnlich wie in dem dritten Aufsatz sollte der Rückgriff auf Prinzipal-Agent-Modelle einen geeigneten theoretischen Ansatz darstellen, um auch die Rolle dieser Institutionen für die substantielle Repräsentation von gesellschaftlich benachteiligten Einwanderergruppen zu untersuchen.

Mit Blick auf potentielle Nachfolgeuntersuchungen des vierten Aufsatzes wären vor allem ländervergleichende Untersuchungen wünschenswert, um Parlamente zu vergleichen, die sich in der Stärke der Fraktionskontrolle über das Plenum unterscheiden. Hier läge die Vermutung nahe, dass dort, wo die Fraktionen weniger Kontrolle über das Plenum ausüben, insgesamt heterogenere migrationspolitische Positionen zum Ausdruck kommen und dadurch möglicherweise polarisierte Debatten entstehen könnten, während in Parlamenten, in denen die Fraktionen mehr Kontrolle über das Plenum haben, eine Großzahl dieser Positionen ungehört bleiben könnten.

\section{Zusammenfassende Schlussbemerkungen}

Diese Dissertation leistet einen Beitrag zum wissenschaftlichen Verständnis der institutionellen Mechanismen politischer Repräsentation in der Einwanderungsgesellschaft. Insbesondere werden folgende Forschungsfragen behandelt. Durch welche institutionellen Mechanismen machen Wähler mit Migrationshintergrund das Angebot von Parteien an Kandidaten mit Migrationshintergrund zur Grundlage ihrer Wahlentscheidungen (Aufsatz 1) und welche Anreize ergeben sich hieraus für rationale Parteien, Kandidaten mit 
Migrationshintergrund aufzustellen und dadurch die deskriptive Repräsentation von Bürgern mit Migrationshintergrund positiv zu beeinflussen (Aufsatz 2)? Durch welche institutionellen Verhaltensanreize und Hemmnisse wird die substantielle Repräsentation von migrations- und integrationsrelevanten Interessen durch individuelle Parlamentarier wie strukturiert (Aufsätze 3 und 4)?

Zur Bearbeitung dieser Fragen beruhen die vier Aufsätze dieser Dissertation auf einem gemeinsamen theoretischen Bezugsrahmen, welcher vom Prinzipal-Agent Modell demokratischer Repräsentation beeinflusst ist. In ihrer Zusammenschau demonstrieren die vier Aufsätze dieser Dissertation damit, dass der institutionelle Rational-Choice Ansatz einen geeigneten Forschungsrahmen darstellt um demokratietheoretisch und gesellschaftlich hoch relevante Fragen der politischen Repräsentation in der Einwanderungsgesellschaft empirisch zu erforschen.

In theoretischer Hinsicht leisten die vier Ausätze dieser Dissertation Beiträge zu der Forschungsliteratur zur migrationspolitischen politischen Repräsentation, weil sie etablierte Annahmen in Frage stellen und gleichzeitig alternative Erklärungen vorschlagen, etwa mit Blick auf die Bedeutung von Wahlsystemen und Kandidatenaufstellungsverfahren für das Verhalten von Wählern mit Migrationshintergrund (Aufsatz 1) und ihre deskriptive Repräsentation (Aufsatz 2), oder hinsichtlich der Rolle von Parlamentariern mit Migrationshintergrund in migrationspolitischen parlamentarischen Debatten (Aufsatz 4). Darüber hinaus werden innovative theoretische Perspektiven eingenommen, die in der existierenden Literatur zur migrationspolitischen Repräsentation bisher fehlen und daher neue Forschungsmöglichkeiten für Folgeprojekte eröffnen, etwa mit Blick auf die institutionelle Beeinflussung von Parlamentariern im Sinne der substantiellen Repräsentation benachteiligter Einwanderergruppen, unabhängig davon, ob Parlamentarier einen Migrationshintergrund haben oder nicht (Aufsatz 3).

Die empirischen Hauptbefunde dieser publikationsbasierten Dissertation lassen sich wie folgt zusammenfassen:

1. Wähler mit Migrationshintergrund nehmen auch unter den Bedingungen parteizentrierter Wahlsysteme die Angebote von Parteien bezogen auf Kandidaten mit Migrationshintergrund wahr und machen diese $\mathrm{zu}$ einer Grundlage ihrer Wahlentscheidung, sofern die Kandidatenaufstellungspraxis eine lokale Verwurzelung der Kandidaten erfordert. 
2. Das Rekrutierungsverhalten von politischen Parteien verhält sich reziprok zum Verhalten der Wähler mit Migrationshintergrund. Selbst unter den Bedingungen von Verhältniswahlsystemen mit geschlossenen Kandidatenlisten haben Parteien einen Anreiz, auf lokale Konzentrationen von Wählern mit Migrationshintergrund mit einem geographisch differenzierten Angebot an Kandidaten mit Migrationshintergrund zu reagieren. Im Aggregat entstehen dadurch geographische Muster deskriptiver Repräsentation.

3. Die substantielle Repräsentation von gesellschaftlich benachteiligten Bürgern mit Migrationshintergrund durch individuelle Parlamentarier wird durch die Einbettung der Parlamentarier in ein Netz von Erwartungen unterschiedlicher Prinzipale strukturiert. Die institutionellen Anreize für Parlamentarier die Interessen von Wahlkreisen mit einem hohem Anteil an Wählergruppen mit Migrationshintergrund und die Interessen ihrer Parteien auf gesamtstaatlicher Ebene zu repräsentieren, werden hauptsächlich durch die Regeln der Kandidatenaufstellung und der parlamentsinternen Organisationsstruktur beeinflusst, welche den Einfluss des Wahlsystems überlagern.

4. Die migrationspolitische Aufmerksamkeit der Reden individueller Parlamentarier wird maßgeblich von den Fraktionen als Gatekeeper des Plenums im Sinne eines institutionellen Hemmnisses strukturiert. Kollektive Repräsentationsmotive können daher die migrationspolitische Aufmerksamkeit der Reden individueller Parlamentarier insgesamt besser erklären als individuelle Repräsentationsmotive.

Methodisch gehen alle Beiträge dabei über den Standard der bisherigen Forschungspraxis hinaus. Durch die Verknüpfung verschiedener Datensätze auf verschiedenen Untersuchungsebenen in Kombination mit dem Einsatz fortgeschrittener statistischer Verfahren, können vor allem die ersten beiden Aufsätze innovative Forschungsperspektiven einnehme. Dies verleiht ihnen ein Alleinstellungsmerkmal in der Forschungsliteratur über die institutionellen Bestimmungsfaktoren der deskriptiven Repräsentation von Bürgern mit Migrationshintergrund. Die letzten beiden Aufsätze verfügen über ein ähnliches Alleinstellungsmerkmal innerhalb der Forschungsliteratur zum migrations- und integrationsrelevanten Verhalten von Parlamentariern, das sich vor allem aus der Nutzbarmachung umfangreicher Textkorpora und dem Einsatz moderner Verfahren der quantitativen Textanalyse begründet. 
Dieses Rahmenpapier hat somit veranschaulicht, dass die vier Aufsätze dieser Dissertation als eigenständige Bestandteile $\mathrm{zu}$ einem kohärenten Forschungsprogramm beitragen, welches auch in Zukunft noch weiter zu bearbeiten sein wird. 
Alba, R. \& Foner, N. (2009). Entering the Precincts of Power: Do National Differences Matter for Immigrant Minority Political Representation. In J. L. Hochschild \& J. H. Mollenkopf (eds.), Bringing Outsiders in: Transatlantic Perspectives on Immigrant Political Incorporation. Ithaca, NY: Cornell University Press, 277-293.

Alba, R. \& Foner, N. (2015). Strangers No More: Immigration and the Challenges of Integration in North America and Western Europe. Princeton: Princeton University Press.

Alvarez, M.R. \& Nagler, J. (1998). When Politics and Models Collide: Estimating Models of Multiparty Elections. American Journal of Political Science 42(1): 55-96.

André, A., Depauw, S. \& Martin, S. (2015). Electoral systems and legislators' constituency effort: The mediating effect of electoral vulnerability. Comparative Political Studies 48(4): 464-496.

Arzheimer, K. \& Evans, J. (2012). Geolocation and voting: Candidate-voter distance effects on party choice in the 2010 UK general election in England. Political Geography 31(5): 301-310.

Aydemir, N. \& Vliegenthart, R. (2016). 'Minority Representatives' in the Netherlands: Supporting, Silencing or Suppressing? Parliamentary Affairs 69(1): 73-92.

Bächtiger, A. (2014). Debate and Deliberation in Legislatures. In K. Strøm, T. Saalfeld \& S. Martin (eds.), The Oxford Handbook of Legislative Studies. Oxford, 145-166.

Bäck, H. \& Debus, M. (2016). Political Parties, Parliaments and Legislative Speechmaking. Basingstoke: Palgrave Macmillan.

Bäck, H. \& Debus, M. (2018). Representing the Region on the Floor: Socioeconomic Characteristics of Electoral Districts and Legislative Speechmaking. Parliamentary Affairs 71(73-102).

Bäck, H., Debus, M. \& Müller, J. (2014). Who Takes the Parliamentary Floor? The Role of Gender in Speech-making in the Swedish Riksdag. Political Research Quarterly 67(3): $504-518$.

Bailer, S. (2011). People's voice or information pool? The role of, and reasons for, 
parliamentary questions in the Swiss parliament. Journal of Legislative Studies 17(3): $302-314$.

Bailer, S. (2017). To use the whip or not: Whether and when party group leaders use disciplinary measures to achieve voting unity. International Political Science Review 39(2): 163-177.

Banducci, S., Donovan, T. \& Karp, J. (2004). Minority Representation, Empowerment and Participation. Journal of Politics 66(2): 534-556.

Barreto, M.A. (2007). ¡S1 SePuede! Latino Candidates and theMobilization of LatinoVoters. American Political Science Review 101(3): 425-441.

Bergh, J. \& Bjorklund, T. (2011a). The Revival of Group Voting: Explaining the Voting Preferences of Immigrants in Norway. Political Studies 59(2): 308-327.

Bergh, J. \& Bjorklund, T. (2011b). Minority representation in Norway - Success at the local level; failure at the national level. In K. Bird, T. Saalfeld \& A. M. Wüst (eds.), The Political Representation of Immigrants and Minorities. New York: Routledge, 128-144.

Bergmann, H., Bailer, S., Ohmura, T., Saalfeld, T. \& Sieberer, U. (2016). Namentliche Abstimmungen im Bundestag 1949 bis 2013: Befunde aus einem neuen Datensatz. Zeitschrift für Parlamentsfragen 47(1): 26-50.

Bird, K. (2011). Patterns of substantive representation of visible minority MPs: Evidence from Canada's House of Commons. In K. Bird, T. Saalfeld \& A. M. Wüst (eds.), The Political Representation of Immigrants and Minorities: Voters, Parties and Parliaments in Liberal Democracies. London and New York: Routledge, 207-229.

Bird, K., Saalfeld, T. \& Wüst, A.M. (2011a). Ethnic Diversity, Political Participation and Representation: a Theoretical Framework. In K. Bird, T. Saalfeld \& A. M. Wüst (eds.), The Political Representation of Immigrants and Minorities. Voters, Parties and Parliaments in Liberal Democracies. London and New York: Routledge, 1-22.

Bird, K., Saalfeld, T. \& Wüst, A.M. (2011b). The Political Representation of Immigrants and Minorities. Voters, Parties and Parliaments in Liberal Democracies. London and New York: Routledge.

Blätte, A. \& Wüst, A.M. (2017). Der migrationsspezifische Einfluss auf parlamentarisches 
Handeln: Ein Hypothesentest auf der Grundlage von Redebeiträgen der Abgeordneten des Deutschen Bundestags 1996-2013. Politische Vierteljahresschrift 58(2): 205-233.

Bloemraad, I. (2013). Accessing the Corridors of Power: Puzzles and Pathways to Understanding Minority Representation. West European Politics 36(3): 652-670.

Bloemraad, I. \& Schönwälder, K. (2013). Immigrant and Ethnic Minority Representation in Europe: Conceptual Challenges and Theoretical Approaches. West European Politics 36(3): 564-579.

Blomgren, M. \& Rozenberg, O. (2012). Legislative roles and legislative studies: the neoinstitutionalist turning point? In M. Blomgren \& O. Rozenberg (eds.), Parliamentary Roles in Modern Legislatures. London and New York: Routledge, 8-36.

Bochsler, D. (2010). Electoral rules and the representation of ethnic minorities in postcommunist democracies. European Yearbook of Minority Issues 7: 153-180.

Bochsler, D. (2011). It is not how many votes you get, but also where you get them. Territorial determinants and institutional hurdles for the success of ethnic minority parties in post-communist countries. Acta Politica 46(3): 217-38.

Bowler, S. \& Farrell, D.M. (1993). Legislator Shirking and Voter Monitoring: Impacts of European Parliament Electoral Systems upon Legislator-Voter Relationships. JCMS: Journal of Common Market Studies 31(1): 45-70.

Bowler, S., Farrell, D.M. \& Katz, R.S. (1999). Party Cohesion, Party Discipline, and Parliaments. In S. Bowler, D. M. Farrell \& R. S. Katz (eds.), Party Discipline and Parliamentary Government. Columbus: Ohio State University Press, 3-22.

Broockman, D.E. (2013). Black Politicians are More Intrinsically Motivated to Advance Blacks' Interests: A Field Experiment Manipulating Political Incentives. American Journal of Political Science 57(3): 521-536.

Bundestag, D. (2019). Dokumentations- und Informationssystem. Letzter Zugriff am 14.02.2019, http://dipbt.bundestag.de/dip21.web/welcome.do?resetNav=y

Bundeswahlleiter. (2013). 5,8 Millionen Deutsche mit Migrationshintergrund sind wahlberechtigt. Letzter Zugriff am 09.03.2016, https://www.bundeswahlleiter.de/de/bundestagswahlen/BTW_BUND_13/presse/W1301 
3_Wahlberechtigte_Migrationshintergrund.html

Cameron, A.C. \& Trivedi, P.K. (2013). Regression analysis of count data. Cambridge:

Cambridge University Press.

Carey, J.M. (2009). Legislative Voting and Accountability. Cambridge: Cambridge University Press.

Carey, J.M. \& Shugart, M.S. (1995). Incentives to cultivate a personal vote: A rank ordering of electoral formulas. Electoral Studies 14(4): 417-439.

Carrubba, C., Gabel, M., Murrah, L., Clough, R., Montgomery, E. \& Schambach, R. (2006). Off the Record: Unrecorded Legislative Votes, Selection Bias and Roll-Call Vote Analysis. British Journal of Political Science 36(04): 691.

Castles, S., Haas, H. de \& Miller, M.J. (2014). The Age of Migration: International Population Movements in the Modern World (5th ed.). Basingstoke: Palgrave Macmillan.

Converse, P.E. \& Pierce, R. (1986). Political Representation in France. Cambridge: MA: Belknap Press.

Cox, G.W. (1997). Making Votes Count. Strategic Coordination in the World's Electoral Systems. Cambridge: Cambridge University Press.

Cox, G.W. (2006). The Organization of Democratic Legislatures. In B. R. Weingast \& D. A. Wittman (eds.), The Oxford Handbook of Political Economy. Oxford: Oxford University Press, 141-161.

Cox, G.W. \& McCubbins, M.D. (2007). Legislative Leviathan. Party Government in the House (2nd ed.). Cambridge: Cambridge University Press.

Dahl, R.A. (1971). Polyarchy: Participation and Opposition. New Haven: Yale University Press.

Dalton, R.J. \& Wattenberg, M.P. (2000). Parties without partisans. Oxford: Oxford University Press.

Damgaard, E. (1995). How Parties control Committee Members. In H. Döring (ed.), Parliaments and Majority Rule in Western Europe. Frankfurt am Main: Campus Verlag, 
$308-324$.

Dancygier, R.M. (2010). Immigration and Conflict in Europe. New York: Cambridge University Press.

Dancygier, R.M. (2014). Electoral Rules or Electoral Leverage? Explaining Muslim Representation in England. World Politics 66(02): 229-263.

Dancygier, R.M. \& Laitin, D.D. (2014). Immigration into Europe: Economic Discrimination, Violence, and Public Policy. Annual Review of Political Science 17: 43-64.

Dancygier, R.M., Lindgren, K.-O., Oskarsson, S. \& Vernby, K. (2015). Why are immigrants underrepresented in politics? Evidence from Sweden. American Political Science Review 109(4): 703-724.

Dancygier, R.M. \& Saunders, E.N. (2006). A New Electorate? Comparing Preferences and Partisanship between Immigrants and Natives. American Journal of Political Science 50(4): 962-981.

Däubler, T. \& Bräuninger, T. (2016). Is Personal Vote-Seeking Behavior Effective? Legislative Studies Quarterly 41(2): 419-444.

Dawson, M.C. (1994). Behind the Mule: Race and Class in African-American Politics. Princeton: Princeton University Press.

Debus, M. \& Bäck, H. (2014). Der Einfluss von Mandatstyp und Wahlkreiseigenschaften auf die inhaltlichen Positionen in Parlamentsreden: Eine Untersuchung anhand der parlamentarischen Debatten im Deutschen Bundestag von 1998 bis 2002. Swiss Political Science Review 20(2): 330-353.

Detterbeck, K. (2016). Candidate Selection in Germany: Local and Regional Party Elites Still in Control? American Behavioral Scientist 60(7): 837-52.

Die Beauftragte der Bundesregierung für Migration, Flüchtlinge und Integration. (2016). 11 . Bericht der Beauftragten der Bundesregierung für Migration, Flüchtlinge und Integration - Teilhabe, Chancengleichheit und Rechtsentwicklung in der Einwanderungsgesellschaft Deutschland.

Diermeier, D. \& Krehbiel, K. (2003). Institutionalism as a Methodology. Journal of Theoretical Politics 15(2): 123-144. 
Donovan, B. (2007). 'Minority’ Representation in Germany. German Politics 16(4): 455-480.

Döring, H. (1995). Time as a Scarce Resource: Government Control of the Agenda. In H. Döring (ed.), Parliaments and Majority Rule in Western Europeule in Western Europe. Frankfurt am Main/ New York: Campus/ St. Martin's Press, 223-246.

Downs, A. (1957). An Economic Theory of Political Action in a Democracy. Journal of Political Economy 65(2): 135-150.

Duverger, M. (1951). Les partis politique. Paris: Armand Colin.

Ezrow, L. (2010). Linking citizens and parties. How electoral systems matter for political representation. Oxford: Oxford University Press.

Fearon, J.D. (1999). Electoral Accountability and the Control of Politicians: Selecting Good Types versus Sanctioning Poor Performance. In A. Przeworski, S. C. Stokes \& B. Manin (eds.), Democracy, Accountability, and Representation. Cambridge: Cambridge University Press, 55-97.

Fenno, R.F. (1973). Congressmen in Committees. Boston: Little, Brown.

Fernandes, J.M., Geese, L. \& Schwemmer, C. (2019). The impact of candidate selection rules and electoral vulnerability on legislative behaviour in comparative perspective. European Journal of Political Research 58(1): 270-291.

Fernandes, J.M., Leston-Bandeira, C. \& Schwemmer, C. (2018). Election proximity and representation focus in party-constrained environments. Party Politics 24(6): 674-685.

Fisher, S.D., Heath, A.F., Sanders, D. \& Sobolewska, M. (2015). Candidate Ethnicity and Vote Choice in Britain. British Journal of Political Science 45(4): 883-905.

Fonseca, S.C. da. (2011). New citizens- new candidates? Candidate selection and the mobilization of immigrant voters in German elections. In K. Bird, T. Saalfeld \& A. M. Wüst (eds.), The Political Representation of Immigrants and Minorities. London and New York: Routledge, 109-127.

Friedman, E. (2005). Electoral System Design and Minority Representation in Slovakia and Macedonia. Ethnopolitics 4(4): 381-96.

Gailmard, S. (2014). Accountability and Principal-Agent Theory. In M. Bovens, R. E. 
Goodin \& T. Schillemans (eds.), The Oxford Handbook of Public Accountability. Oxford: Oxford University Press, 90-105.

Gaines, B.J. (1998). The Impersonal Vote? Constituency Service and Incumbency Advantage in British Elections, 1950-92. Legislative Studies Quarterly 23(2): 167-195.

Gallagher, M. (1988). Introduction. In M. Gallagher \& M. Marsh (eds.), Candidate Selection in Comparative Perspective. The Secret Garden of Politics. London: Sage, 1-19.

Gallagher, M. \& Marsh, M. (1988). The Secret Garden of Politics: Candidate Selection in Comparative Perspective. London: Sage Publications.

Gallagher, M. \& Mitchell, P. (2005). Introduction to Electoral Systems. In M. Gallagher \& P. Mitchell (eds.), The Politics of Electoral Systems. Oxford: Oxford University Press, $3-$ 23.

Garbaye, R. (2000). Ethnic minorities, cities and Institutions: A comparisonof the modes of management of ethnic diversity of a French and British city. In R. Koopmans \& P. Statham (eds.), Challenging Immigration and Ethnic Relations Politics. Oxford: Oxford University Press, 283-311.

Geese, L., Goldbach, W. \& Saalfeld, T. (2015). Mobility and Representation: Legislators of Non-European Origin in the British House of Commons, 2001-2015. In S. Panter (ed.), Jahrbuch für europäische Geschichte. European History Yearbook. Berlin and Boston: De Gruyter Oldenbourg, 83-109.

Geese, L., Saalfeld, T., Schwemmer, C. \& van der Pas, D. (im Erscheinen). Immigrants, Immigration and Integration as Topics in Parliamentary Questions - Similarities and Differences in the Discursive Environment of Representation. In L. Morales \& T. Saalfeld (eds.), Pathways to Power: The Political Representation of Citizens of Immigrant Origin in Europe. Oxford: Oxford University Press.

Gerring, J. \& Seawright, J. (2007). Techniques for Choosing Cases. In J. Gerring (ed.), Case Study Research. Principles and Practices. Cambridge: Cambridge University Press, 86150.

Giger, N. \& Klüver, H. (2015). Voting Against Your Constituents? How Lobbying Affects Representation. American Journal of Political Science 60(1): 190-205. 
Goerres, A., Spies, D.C. \& Kumlin, S. (2018). The Electoral Supporter Base of the Alternative for Germany. Swiss Political Science Review 24(3): 246-269.

Green-Pedersen, C. \& Otjes, S. (2017). A hot topic? Immigration on the agenda in Western Europe. Party Politics. Online Version: https://doi.org/10.1177/1354068817728211

Grimmer, J. \& Stewart, B.M. (2013). Text as Data: The Promise and Pitfalls of Automatic Content Analysis Methods for Political Texts. Political Analysis 21(3): 267-297.

Gschwend, T. \& Zittel, T. (2015). Do constituency candidates matter in German Federal Elections? The personal vote as an interactive process. Electoral Studies 39: 338-349.

Hainmueller, J. \& Hopkins, D.J. (2014). Public Attitudes Toward Immigration. Annual Review of Political Science 17: 225-49.

Hainmueller, J. \& Kern, H.L. (2008). Incumbency as a source of spillover effects in mixed electoral systems: Evidence from a regression-discontinuity design. Electoral Studies 27(2): 213-227.

Hall, P.A. \& Taylor, R.C.R. (1996). Political Science and the Three New Institutionalisms. Political Studies 44(5): 936-957.

Hänni, M. (2017). Presence, Representation, and Impact: How Minority MPs Affect Policy Outcomes. Legislative Studies Quarterly 42(1): 97-130.

Hazan, R.Y. (1999). Constituency interests without constituencies: the geographical impact of candidate selection on party organization and legislative behavior in the 14th Israeli Knesset, 1996-99. Political Geography 18: 791-811.

Hazan, R.Y. \& Rahat, G. (2010). Democracy within Parties: Candidate Selection Methods and Their Political Consequences. Oxford: OUP Oxford.

Helbling, M. (2014). Framing Immigration in Western Europe. Journal of Ethnic and Migration Studies 40(1): 21-41.

Huber, J.D. \& Powell, G.B. (1994). Congruence between citizens and policymakers in two visions of liberal democracy. World Politics 46: 291-326.

Ismayr, W. (1992). Der Deutsche Bundestag. Funktionen - Willensbildung - Reformansätze. Opladen: Leske and Budrich. 
Ismayr, W. (2012). Der Deutsche Bundestag. Wiesbaden: VS Verlag für Sozialwissenschaften.

Iversflaten, E. (2005). Threatened by diversity: Why restrictive asylum and immigration policies appeal to western Europeans. Journal of Elections, Public Opinion and Parties 15(1): $21-45$.

Karlsen, R. \& Narud, H.M. (2013). Nominations, Campaigning and Representation: How the Secret Garden of Politics Determines the Style of Campaigning and Roles of Representation. In P. Essaiasson \& H. M. Narud (eds.), Between-Election Democracy. The Representative Relationship after Election Day. Colchester: ECPR Press, 77-101.

Karvonen, L. (2010). The personalisation of politics: A study of parliamentary democracies. Colchester: ECPR Press.

Katz, R.S. (2001). The Problem of Candidate Selection and Models of Party Democracy. Party Politics 7(3): 277-296.

Keh, J.F. (2015). The Centralisation of Parliamentary Policy Statements in Western European Parliaments. West European Politics 38(5): 1086-1105.

King, G., Keohane, R.O. \& Verba, S. (1994). Designing Social Inquiry. Scientific Inference in Qualitative Research. Princeton: Princeton University Press.

Kittilson, M.C. \& Tate, K. (2005). Political parties, minorities and elected office : comparing opportunities for inclusion in the United States and Britain. In C. Wolbrecht \& R. E. Hero (eds.), The Politics of Democratic Inclusion. Philadelphia: Temple University Press, 163-185.

Kortmann, M. \& Stecker, C. (2019). Party competition and immigration and integration policies: a comparative analysis. Comparative European Politics 17(1): 72-91.

Kostadinova, T. (2007). Ethnic and women's representation under mixed election systems. Electoral Studies 26: 418-31.

Kroeber, C. (2018). Growing numbers, growing influence? A comparative study of policy congruence between parliaments and citizens of immigrant origin. European Journal of Political Research 57(4): 900-918.

Krouwel, A. (2012). Party Transformations in European Democracies. Albany: State 
University of New York Press.

Landa, J., Copeland, M. \& Grofman, B. (1995). Ethnic voting patterns: a case study of Metropolitan Toronto. Political Geography 14(5): 435-449.

Latner, M. \& McGann, A. (2005). Geographical representation under proportional representation: The cases of Israel and the Netherlands. Electoral Studies 24(4): 709734.

Laurence, J. \& Maxwell, R. (2012). Political Parties and Diversity in Western Europe. In T. Givens \& R. Maxwell (eds.), Immigrant Politics: Race and Representation in Western Europe. Boulder: Lynne Rienner Publishers, 33-52.

Laver, M. \& Shepsle, K.A. (1996). Making and Breaking Governments. Cabinets and Legislatures in Parliamentary Democracies. Cambridge: Cambridge University Press.

Leijenaar, M. \& Niemöller, K. (1997). The Netherlands. In P. Norris (ed.), Passages to power. Legislative recruitment in advanced democracies. Cambridge: Cambridge University Press, 114-136.

Lijphart, A. (1994). Electoral systems and party systems. Oxford and New York: Oxford University Press.

Lijphart, A. (1999). Patterns of Democracy. Yale University Press.

Lijphart, A. (2004). Constitutional Design for Divided Societies. Journal of Democracy 15(2): 96-109.

Long, J.S. (1997). Regression Models for Categorical and Limited Dependent Variables. Thousand Oaks: Sage.

Lupia, A. \& McCubbins, M.D. (2000). Representation or abdication? How citizens use institutions to help delegation succeed. European Journal of Political Research 37(3): 291-307.

Mair, P., Müller, W.C. \& Plasser, F. (2004). Introduction: Electoral Challenges and Party Responses. In P. Mair, W. C. Müller \& F. Plasser (eds.), Political Parties and Electoral Change: Party Responses to Electoral Markets. London: Sage, 1-19.

Manin, B., Przeworski, A. \& Stokes, S.C. (1999). Elections and Representation. In A. 
Przeworski, S. C. Stokes \& B. Manin (eds.), Democracy, Accountability, and Representation. Cambridge: Cambridge University Press, 29-54.

Manow, P. (2013). Mixed Rules, Different Roles? An Analysis of the Typical Pathways into the Bundestag and of MPs' Parliamentary Behaviour. The Journal of Legislative Studies 19(3): 287-308.

Manow, P. (2015). Mixed Rules, Mixed Strategies. Candidates and Parties in Germany's Electoral System. Colchester: ECPR Press.

Mansbridge, J. (1999). Should Blacks Represent Blacks and Women Represent Women? A Contingent "Yes". The Journal of Politics 61(03): 628-657.

Mansbridge, J. (2003). Rethinking representation. American Political Science Review 97(4): $515-528$.

Mansbridge, J. (2009). A "Selection Model” of Political Representation*. The Journal of Political Philosophy 17(4): 369-398.

Martin, S. (2010). Electoral Rewards for Personal Vote Cultivation under PR-STV. West European Politics 33(2): 369-380.

Martin, S. (2011). Parliamentary Questions, the Behaviour of Legislators, and the Function of Legislatures: An Introduction. The Journal of Legislative Studies 17(3): 259-270.

Martin, S. (2014). Why electoral systems don't always matter: The impact of 'mega-seats' on legislative behaviour in Ireland. Party Politics 20(3): 467-79.

Mayhew, D.R. (1974). Congress: The Electoral Connection. New Haven and London: Yale University Press.

McConnaughy, C.M., White, I.K., Leal, D.L. \& Casellas, J.P. (2010). A Latino on the Ballot: Explaining Coethnic Voting Among Latinos and the Response of White Americans. The Journal of Politics 72(4): 1199-1211.

McDermott, M.L. (1997). Voting Cues in Low-Information Elections: Candidate Gender as a Social Information Variable in Contemporary United States Elections. American Journal of Political Science 41(1): 270.

McDermott, M.L. (1998). Race and Gender Cues in Low-Information Elections. Political 
Research Quarterly 51(4): 895-918.

McLean, I. (1991). Forms of representation and systems of voting. In D. Held (ed.), Political Theory Today. Cambridge: Polity Press.

Messina, A.M. (2007). The Logics and Politics of Post-WWII Migration to Western Europe. Cambridge: Cambridge University Press.

Miller, B. \& Stecker, C. (2008). Consensus by Default? Interaction of Government and Opposition Parties in the Committees of the German Bundestag. German Politics 17(3): 305-322.

Mitchell, P. (2000). Voters and their representatives: electoral institutions and delegation in parliamentary democracies. European Journal of Political Research 37: 335-351.

Morton, R.B. (1999). Methods and Models. A Guide to the Empirical Analysis of Formal Models in Political Science. Cambridge: Cambridge University Press.

Moser, R.G. (2008). Electoral Systems and the Representation of Ethnic Minorities: Evidence from Russia. Comparative Politics 40(3): 273-292.

Moser, R.G. \& Scheiner, E. (2005). Strategic Ticket Splitting and the Personal Vote in Mixed-Member Electoral Systems. Legislative Studies Quarterly 30(2): 259-276.

Moser, R.G. \& Scheiner, E. (2012). Electoral Systems and Political Context. How the Effects of Rules Vary Across New and Established Democracies. Cambridge: Cambridge University Press.

Moses, J.W. (2018). introduction: migration to Europe. European Political Studies 17(1): $122-127$.

Müller, W.C. (2000). Political parties in parliamentary democracies: making delegation and accountability work. European Journal of Political Research 37(3): 309-33.

Nergiz, D.D. (2014). I Long for Normality: A Study on German Parliamentarians with Migration Backgrounds. Wiesbaden: Springer.

Norris, P. (2004). Electoral Engineering: Voting Rules and Political Behavior. Cambridge: Cambridge University Press.

Norris, P. \& Lovenduski, J. (1995). Political Recuitment: Gender, Race, and Class in the 
British Parliament. Cambridge: Cambridge University Press.

North, D.C. (1990). Institutions, Institutional Change, and Economic Performance. New York: Cambridge University Press.

OECD \& EU. (2015). Indicators of Immigrant Integration 2015: Settling In. Paris: OECD Publishing.

Papke, L.E. \& Wooldridge, J.M. (1996). Econometric methods for fractional response variables with an application to $401(\mathrm{~K})$ plan participation rated. Journal of Applied Econometrics 11(6): 619-632.

Pappi, F.U., Kurella, A.-S. \& Bräuninger, T. (2017). Erststimmen generierende Merkmale der Direktkandidaten bei Bundestagswahlen. Zeitschrift für Politikwissenschaft 27(3): 305326.

Phillips, A. (1995). The Politics of Presence. Oxford: Clarendon Press.

Phillips, A. (1998). Democracy and representation: Or, why should it matter who our representatives are? In A. Phillips (ed.), Feminism and politics. Oxford: Oxford University Press.

Pitkin, H.F. (1967). The Concept of Representation. Berkeley: University of California Press.

Preece, J.R. (2014). How The Party Can Win in Personal Vote Systems: The 'Selectoral Connection' and Legislative Voting in Lithuania. Legislative Studies Quarterly 39(2): $147-167$.

Proksch, S.-O. \& Slapin, J. (2015). The Politics of Parliamentary Debate. Cambridge: Cambridge University Press.

Proksch, S.-O. \& Slapin, J.B. (2012). Institutional Foundations of Legislative Speech. American Journal of Political Science 56(3): 520-537.

Rahat, G. \& Hazan, R.Y. (2001). Candidate selection methods - An analytical framework. Party Politics 7(3): 297-322.

Rahat, G. \& Kenig, O. (2018). From Party Politics to Personalized Politics. Party Change and Political Personalization in Democracies. Oxford: Oxford University Press.

Rattinger, H., Roßteutscher, S., Schmidt-Beck, R., Weßels, B. \& Wolf, C. (2014). Vor- und 
Nachwahl-Querschnitt (GLES 2013). ZA5702 Datenfile Version 2.0.0. Köln: GESIS Datenarchiv.

Redmond, P. \& Regan, J. (2015). Incumbency advantage in a proportional electoral system: A regression discontinuity analysis of Irish elections. European Journal of Political Economy 38: 244-256.

Reilly, B. (2002). Electoral Systems for Divided Societies. Journal of Democracy 13(2): 15670.

Reiser, M. (2014). The universe of group representation in Germany: Analysing formal and informal party rules and quotas in the process of candidate selection. International Political Science Review 35(1): 55-66.

Renwick, A. \& Pilet, J.-B. (2016). Faces on the Ballot. The Personalisation of Electoral Systems in Europe. Oxford: Oxford University Press.

Reynolds, A. (2006). Electoral systems and the protection and participation of minorities. London: Minority Rights Group International.

Roberts, G. (1988). The German Federal Republic: the two-lane route to Bonn. In M. Gallagher \& M. Marsh (eds.), Candidate Selection in Comparative Perspective. The Secret Garden of Politics. London: Sage, 94-118.

Roberts, M.E., Stewart, B.M., Tingley, D., Lucas, C., Leder-Luis, J., Gadarian, S.K., ... Rand, D.G. (2014). Structural topic models for open-ended survey responses. American Journal of Political Science 58(4): 1064-1082.

Rozenberg, O. \& Martin, S. (2011). Questioning Parliamentary Questions. The Journal of Legislative Studies 17(3): 394-404.

Ruedin, D. (2009). Ethnic Group Representation in a Cross-National Comparison. The Journal of Legislative Studies 15(4): 335-354.

Ruedin, D. (2013). Why Aren't They There? The Political Representation of Women, Ethnic Groups and Issue Positions in Legislatures. Colchester: ECPR Press.

Ruedin, D. \& Morales, L. (2017). Estimating Party Positions on Immigration. Party Politics. Online version: https://doi.org/10.1177/1354068817713122 
Russo, F. (2011). The Constituency as a Focus of Representation: Studying the Italian Case through the Analysis of Parliamentary Questions. The Journal of Legislative Studies 17(3): 290-301.

Russo, F. \& Wiberg, M. (2010). Parliamentary Questioning in 17 European Parliaments: Some Steps towards Comparison. The Journal of Legislative Studies 16(2): 215-232.

Rydgren, J. (2008). Immigration sceptics, xenophobes or racists? Radical right-wing voting in six West European countries. European Journal of Political Research 47(6): 737-765.

Saalfeld, T. (1995). Parteisoldaten und Rebellen. Fraktionen im Deutschen Bundestag 19491990. Wiesbaden: Springer.

Saalfeld, T. (2000). Bureaucratisation, coordination and competition. In K. Heidar \& R. Koole (eds.), Parliamentary Party Groups in European Democracies. London and New York: Routledge, 23-38.

Saalfeld, T. (2005). Germany: Stability and Strategy in a Mixed-Member Proportional System. In G. Michael \& P. Mitchell (eds.), The Politics of Electoral Systems. Oxford and New York: Oxford University Press, 209-28.

Saalfeld, T. (2011). Parliamentary Questions as Instruments of Substantive Representation: Visible Minorities in the UK House of Commons, 2005-10. The Journal of Legislative Studies 17(3): 271-289.

Saalfeld, T. \& Bischof, D. (2013). Minority-ethnic MPs and the substantive representation of minority interests in the house of commons, 2005-2011. Parliamentary Affairs 66(2): $305-328$.

Saalfeld, T. \& Strøm, K.W. (2014). Political Parties and Legislators. In S. Martin, T. Saalfeld \& K. Strøm (eds.), Oxford Handbook of Legislative Studies. Oxford: Oxford University Press, 371-398.

Sanders, D., Heath, A.F., Fisher, S.D. \& Sobolewska, M. (2014). The Calculus of Ethnic Minority Voting in Britain. Political Studies 62: 230-51.

Scharpf, F.W. (1999). Regieren in Europa: effektiv und demokratisch. Frankfurt am Main: Campus Verlag.

Scheve, K.F. \& Slaughter, M.J. (2001). Labor Market Competition and Individual Preferences 
Over Immigration Policy. The Review of Economics and Statistics 83(1): 133-145.

Schoen, H. (2014). Wahlsystemforschung. In J. W. Falter \& H. Schoen (eds.), Handbuch Wahlforschung. Wiesbaden: Springer VS, 769-823.

Schönwälder, K. (2013). Immigrant Representation in Germany’s Regional States: The Puzzle of Uneven Dynamics. West European Politics 36(3): 634-651.

Schüttemeyer, S.S. (1994). Hierarchy and Efficiency in the Bundestag: the German Answer to Instituionalizing Parliament. In G. W. Copeland \& S. C. Patterson (eds.), Parliaments in the modern world: changing institutions. Ann Arbor: University of Michigan Press, 2958.

Schüttemeyer, S.S. \& Sturm, R. (2005). Der Kandidat - das (fast) unbekannte Wesen: Befunde und Überlegungen zur Aufstellung der Bewerber zum Deutschen Bundestag. Zeitschrift für Parlamentsfragen 36(3): 539-553.

Searing, D.D. (1991). Roles, Rules, and Rationality in the New Institutionalism. American Political Science Review 85(4): 1239-1260.

Shepsle, K.A. (2006). Rational Choice Institutionalism. In R. A. W. Rhodes, S. A. Binder \& B. A. Rockman (eds.), The Oxford Handbook of Political Institutions. Oxford: Oxford University Press, 23-38.

Shugart, M.S. (2001). 'Extreme' Electoral Systems and the Appeal of the Mixed-Member Alternative. In M. S. Shugart \& M. P. Wattenberg (eds.), Mixed-Member Electoral Systems: The Best of Both Worlds? Oxford: Oxford University Press, 25-51.

Shugart, M.S. (2005). Comparative Electoral Systems Research: The Maturation of a Field and New Challenges Ahead. In M. Gallagher \& P. Mitchell (eds.), The Politics of Electoral Systems. Oxford: Oxford University Press, 25-55.

Shugart, M.S., Valdini, M.E. \& Suominen, K. (2005). Looking for Locals: Voter Information Demands and Personal Vote-Earning Attributes of Legislators under Proportional Representation. American Journal of Political Science 49(2): 437-449.

Shugart, M.S. \& Wattenberg, M.P. (2001). Mixed-Member Electoral Systems: A Definition and Typology. In M. S. Shugart \& M. P. Wattenberg (eds.), Mixed-Member Electoral Systems: The Best of Both Worlds? Oxford: Oxford University Press, 9-24. 
Sieberer, U. (2006). Party unity in parliamentary democracies: A comparative analysis. The Journal of Legislative Studies 12(2): 150-178.

Sieberer, U. (2010). Behavioral consequences of mixed electoral systems: Deviating voting behavior of district and list MPs in the German Bundestag. Electoral Studies 29(3): 484496.

Sieberer, U. \& Müller, W.C. (2017). Aiming higher: the consequences of progressive ambition among MPs in European parliaments. European Political Science Review 9(1): $27-50$.

Siefken, S.T. (2010). Parlamentarische Frageverfahren - Symbolpolitik oder wirksames Kontrollinstrument? Zeitschrift für Parlamentsfragen 41(1): 18-36.

Smith, T.H. (2013). Are you sitting comfortably? Estimating incumbency advantage in the UK: 1983-2010-A research note. Electoral Studies 32(1): 167-173.

Snyder, J.M.J. \& Ting, M. (2002). An Informational Rational for Political Parteis. American Journal of Political Science 46(1): 90-110.

Sobolewska, M., McKee, R. \& Campbell, R. (2018). Explaining motivation to represent: how does descriptive representation lead to substantive representation of racial and ethnic minorities? West European Politics. Online Version: https://doi.org/10.1080/01402382.2018.1455408

Statistisches Bundesamt. (2018). Bevölkerung und Erwerbstätigkeit. Bevölkerung mit Migrationshintergrund - Ergebnisse des Mikrozensus 2017 -. Letzter Zugriff am 04.03.2019, https://www.destatis.de/DE/Publikationen/Thematisch/Bevoelkerung/MigrationIntegrati on/Migrationshintergrund2010220177004.pdf?_blob=publicationFile

Steffani, W. (1967). Amerikanischer Kongreß und deutscher Bundestag - ein Vergleich. In K. Kluxen (ed.), Parlamentarismus. Köln: Kiepenheuer \& Witsch.

Street, A. (2014). Representation Despite Discrimination: Minority Candidates in Germany. Political Research Quarterly 67(2): 374-385.

Strøm, K. (1997). Rules, Reasons and Routines: Legislative Roles in Parliamentary Democracies. In W. C. Müller \& T. Saalfeld (eds.), Members of Parliament in Western 
Europe: Roles and Behaviour. London: Frank Cass, 155-174.

Strøm, K. (1998). Parliamentary Committes in European Democracies. Journal of Legislative Studies 4(1): 21-59.

Strøm, K. (2000). Delegation and accountability in parliamentary democracies. European Journal of Political Research 37(3): 261-289.

Strøm, K. \& Müller, W.C. (1999). Political Parties and Hard Choices. In W. C. Müller \& K. Strøm (eds.), Policy, Office or Votes? How Political Parteis in Western Europe Make Hard Decisions. Cambridge: Cambridge University Press, 1-35.

Swain, C. (1993). Black Faces, Black Interests - The Representation of African Americans in Congress. London: Harvard University Press.

Tavits, M. (2011). Power within Parties: The Strength of the Local Party and MP Independence in Postcommunist Europe. American Journal of Political Science 55(4): 923-936.

Teney, C., Jacobs, D., Rea, A. \& Delwit, P. (2010). Ethnic voting in Brussels: Voting patterns among ethnic minorities in Brussels (Belgium) during the 2006 local elections. Acta Politica 45(3): 273-97.

Togeby, L. (2008). The political representation of ethnic minorities: Denmark as a deviant case. Party Politics 14(3): 325-343.

Trounstine, J.L. \& Valdini, M.E. (2008). The Context Matters: The Effect of Single Member vs At-Large Districts on City Council Diversity. American Journal of Political Science 68(4): 554-569.

Urbinati, N. (2011). Representative democracy and its critics. In S. Alonso, J. Keane \& W. Merkel (eds.), The Future of Representative Democracy. Cambridge: Cambridge University Press, 23-49.

Valdini, M.E. (2012). A deterrent to diversity: The conditional effect of electoral rules on the nomination of women candidates. Electoral Studies 31: 740-749.

Volkens, A., Lehmann, P., Matthieß, T., Merz, N., Regel, S. \& Werner, A. (2015). The Manifesto Data Collection. Manifesto Project (MRG / CMP / MARPOR). Version 2015a. Berlin: Wissenschaftszentrum Berlin für Sozialforschung (WZB). 
Wallack, J.S., Gaviria, A., Panizza, U. \& Stein, E. (2003). Particularism around the world. The World Bank Economic Review 17(1): 133-143.

Wessels, B. (1993). Politische Repräsentation als Prozeß gesellschaftlich-parlamentarischer Kommunikation. In D. Herzog, H. Rebenstorf \& B. Wessels (eds.), Parlament und Gesellschaft - eine Funktionsanalyse der repräsentativen Demokratie. Opladen: Westdeutscher Verlag, 99-137.

Wessels, B. (1997). Germany. In P. Norris (ed.), Passages to power. Legislative recruitment in advanced democracies. Cambridge: Cambridge University Press, 76-97.

Wiberg, M. \& Koura, A. (1994). The Logic of Parliamentary Questioning. In M. Wiberg (ed.), Parliamentary Control in the Nordic Countries. Helsinki: Finnish Political Science Association, 19-44.

Wüst, A.M. (2014a). A Lasting Impact? On the Legislative Activities of Immigrant-origin Parliamentarians in Germany. Journal of Legislative Studies (May): 37-41.

Wüst, A.M. (2014b). Immigration into Politics: Immigrant-origin Candidates and Their Success in the 2013 Bundestag Election. German Politics \& Society 32(3): 1-15.

Wüst, A.M. (2016). Incorporation beyond Cleavages? Parties, Candidates and Germany's Immigrant-Origin Electorate. German Politics 25(2): 414-432.

Wüst, A.M. \& Saalfeld, T. (2010). Abgeordnete mit Migrationshintergrund im Vereinigten Königreich, Frankreich, Deutschland und Schweden: Opportunitäten und Politikschwerpunkte. Politische Vierteljahresschrift, Sonderheft 44: 312-333.

Zingher, J.N. \& Farrer, B. (2016). The electoral effects of the descriptive representation of ethnic minority groups in Australia and the UK. Party Politics 22(6): 691-704.

Zittel, T. \& Gschwend, T. (2008). Individualised Constituency Campaigns in Mixed-Member Electoral Systems: Candidates in the 2005 German Elections. West European Politics 31(5): 978-1003.

Zollinger, D. \& Bochsler, D. (2012). Minority representation in a semi-democratic regime: the Georgian case. Democratization 19(4): 611-41. 


\section{Aufsatz 1}

Geese, L. Do immigrant-origin candidates attract immigrant-origin voters in party-centred electoral systems? Evidence from Germany. Acta Polit (2018). https://doi.org/10.1057/s41269-018-0126-9

Das folgende Manuskript ist der Pre-print des Aufsatzes, der von der Redaktion der Fachzeitschrift Acta Politica (ISSN: 0001-6810) zur Publikation angenommen wurde. 
Title.

Immigrant group voting despite party-centred electoral rules: evidence from Germany

\begin{abstract}
.
A burgeoning literature on minority representation asks whether immigrant-origin voters are more likely to vote for candidates of immigrant-origin (CIOs) than for native candidates, thus giving parties incentives to nominate CIOs. At present, however, evidence of such a link comes exclusively from candidate-centred electoral systems. The present study intends to narrow this gap by studying the influence of CIOs on immigrant-origin citizens' party vote choices in Germany, a more party-centred electoral environment. An empirical analysis of opinion survey and candidate data from the 2013 Bundestag election suggests that immigrant group voting is considerable in Germany. This paper is thus the first one to show that immigrant group voting is a significant factor in a party-centred electoral environment.
\end{abstract}

Keywords.

Immigrant voting, Electoral rules, Representation, Immigrant Candidates 


\section{Introduction}

In the past few decades, large scale immigration has diversified the voter market for political parties in Western democracies. In response to that, a burgeoning body of literature shows that Western parties have increased their attention towards growing immigrant-origin electorates, not just through programmatic offers and ethnically targeted campaigning, but also by promoting candidates of immigrant-origin (CIOs) for elected office (Alba \& Foner, 2015; Bird et al., 2011a; Bloemraad \& Schönwälder, 2013; Fonseca, 2011; Kittilson \& Tate, 2005; Laurence \& Maxwell, 2012; Wüst, 2016). An important assumption underlying this literature is that citizens of immigrant-origin are more likely to vote for CIOs than for native candidates, such that parties have incentives to nominate CIOs as a tool to harvest the immigrant vote. In this paper, this relationship is called the "immigrant group voting" hypothesis.

At present, however, this hypothesis seems to be exclusively supported by studies conducted in electoral systems operating either in single-member districts, like the UK or Canada (SMD) (Barreto, 2007; Fisher et al., 2015; Landa et al., 1995; Zingher \& Farrer, 2016), or in systems using open-list proportional representation (PR), like local elections in Belgium or Norway (Bergh \& Bjorklund, 2011; Teney et al., 2010). Less is known, by contrast, about immigrant group voting in more party-centred electoral systems, like in the Dutch or Israeli closed-list PR or the German mixed-member proportional (MMP) systems. That may be, because incentives for candidate-based voting are strong in the former but weak in the latter type of electoral systems as the personal voting literature famously posits (e.g. Carey \& Shugart, 1995; Shugart et al., 2005). Therefore, immigrant group voting should be less likely to be of significance in party-centred systems.

In this paper I argue that immigrant group voting exists in party-centred electoral contexts nonetheless. In my argumentation, I highlight candidates' local campaigning activities in 
party-centred electoral systems as a mechanism that raises immigrant-origin voters' awareness of CIOs' presence and thereby increases the appeal of CIOs' parties. Consequently, in this paper I examine immigrant group voting in a "heavily party-oriented system" (Moser \& Scheiner, 2005): Germany's MMP system. In order to study how immigrants' party choices vary with the local presence of CIOs, a series of alternative-specific multinomial logit models are applied utilising a combined dataset of self-collected candidate data and survey data from the 2013 German Longitudinal Election Studies (GLES) (Rattinger et al., 2014). The main finding from this analysis suggests that the local presence of CIOs influences the PR votes of immigrant-origin citizens considerably. Therefore, this paper contributes to the literature by showing that immigrant group voting is not contradictory to party-centred electoral environments. The common assumption that CIOs can help parties to increase their vote returns from the immigrant-origin electorate is thus substantiated for electoral systems that provide weak incentives for personal voting.

\section{The puzzle of immigrant group voting in a party-centred context}

According to social identity theory, behavioural homogeneity within social groups is a function of the degree of societies' stratification: the more the social mobility of individual group members depends on that of their group, the more homogenous group members will behave socially and politically (Tajfel \& Turner, 1986, p. 10). Given that Western countries of immigration are characterised by strong social and economic inequalities separating most immigrants and their descendants from the native majority population (Alba \& Foner, 2015; Bird et al., 2011a; Bloemraad \& Schönwälder, 2013; Strijbis, 2014; Zingher \& Farrer, 2016), it is plausible to assume that immigrant-origin residents constitute a meaningful reference group for homogeneous group behaviour in light of social identity theory.

This view is also supported by previous research on immigrants' electoral behaviour. Social identity concepts have greatly helped solve the puzzle of immigrant-origin voters' strong and 
consistent support for parties of the political left (Bergh \& Bjorklund, 2011; Bird et al., 2011b; Dancygier \& Saunders, 2006; Messina, 2007; Sanders et al., 2014; Strijbis, 2014; Teney et al., 2010; Wüst, 2004) ${ }^{1}$. This line of research suggests that neither immigrant-origin voters' location in ideological space nor their class belonging can fully account for the left party bias. Rather, immigrants' identification with group interests and their perception of a "linked fate" with other group members seems to be complementary with left-wing parties' greater credibility to improve the living conditions of immigrants and fight discrimination in general, thus leading to an overwhelming support for these parties among disadvantaged immigrant groups (Dancygier \& Saunders, 2006; Messina, 2007; Sanders et al., 2014; Strijbis, 2014).

It is thus plausible to assume that group-based considerations may also be the driving force behind immigrant group voting. Minority candidates provide readily available voting cues to voters, that is, they are generally perceived to be more compassionate and trustworthy than other candidates in representing the political interests of disadvantaged groups (e.g. Banducci et al., 2004; Landa et al., 1995; McDermott, 1998). According to McConnoughy and coauthors, such candidate cues interact heavily with "linked fate" perceptions (McConnaughy et al., 2010). Similar to the impact of "linked fate" perceptions on immigrants' left party support, immigrant-origin voters may be also more likely to support a CIO than a native candidate in order to improve the political representation of the interests of disadvantaged groups more generally. In line with this, existing research on immigrant group voting in Western democracies confirms by and large that immigrant voters prefer CIOs over native candidates even if the $\mathrm{CIO}$ has a different immigrant background than the voter (Bergh \& Bjorklund, 2011; McConnaughy et al., 2010; Teney et al., 2010; Zingher \& Farrer, 2016).

\footnotetext{
${ }^{1}$ An exceptions to this pattern applies, however, to Central and Eastern European immigrants in Germany, whose troubled history with communist parties has made them overwhelmingly supporters of the Christian Democrats (Wüst, 2004).
} 
However, this evidence is entirely based on research conducted in candidate-centred electoral systems; therefore it is based on evidence in most-likely electoral system contexts. In candidate-centred systems, like SMD or open-list PR, voters have strong incentives to make candidate-based voting decisions, while this incentive should be widely missing in partycentred systems, like closed-list PR or MMP (Carey \& Shugart, 1995). Here, by contrast, voters have strong incentives to make party-based voting decisions, because they can only support or defect a list of candidates as a whole, which provides little leeway to influence the electoral fate of individual candidates (Shugart et al., 2005). Since immigrant group voting is candidate-based by definition, party-centred electoral systems hence provide for a least-likely electoral context to find support for this hypothesis (see Gerring \& Seawright, 2007).

This paper argues, however, that other mechanisms than electoral systems' incentives for personal voting can provide for a link between immigrants' voting behaviour and the local presence of a CIO. I highlight parties' incentives to conduct localised modes of campaigning. Over the past few decades, campaigning strategies in party-centred electoral systems have become more professional and sophisticated in targeting specific "focus groups" (Strömbäck, 2009). Given that immigrant-origin residents are geographically concentrated, it makes sense for parties seeking the immigrant vote to redirect their campaign resources to areas of high ethnic density (Sobolewska et al., 2013). According to Latner and McGann, list candidates can be viewed as such geographically distributable campaigning resources (Latner \& McGann, 2005, p. 713). Indeed, candidates are usually locally connected even in partycentred electoral systems. Informal rules of legislative recruitment typically require that political aspirants gather political experiences in local government and party offices before they can become parliamentary candidates. Even as elected parliamentarians, they often hold local mandates simultaneously, thus ensuring their rootedness and visibility in the local community. Real world examples of these empirical patterns in party-centred electoral 
environments extend to, for example, Germany (Wessels, 1997), Israel (Hazan, 1999), Italy (Russo, 2011), the Netherlands (Leijenaar \& Niemöller, 1997) and Portugal (Fernandes et al., 2017).

For these reasons, I argue that individual candidates have links to local constituencies even in closed party lists, wherefore parties can urge their CIOs to campaign for the party label in their local home areas. Here, they manage campaigning activities for the party such as canvassing ethnic community groups, door-stepping immigrant-origin voters, meeting them face-to-face in the pedestrian area or by giving interviews in local newspapers. These campaigning activities will raise local immigrant-origin voters' awareness of individual CIOs despite party-centred electoral rules. Voting cues of improved group representation associated with the local presence of a CIO increase thus the likelihood of supporting his/her party list.

Thus, my first two hypotheses are:

H1: Immigrant-origin voters are more likely to support local CIOs' parties than other parties in party-centred electoral systems

H2: Immigrant-origin voters are more likely to support local CIOs' parties than other parties, conditional on CIOs' local campaigning activities

CIOs' party endorsement can be assumed to be an important intervening factor of CIOs' perceived credibility to advance disadvantaged group interests. That is, it is plausible to assume that the CIO voting cue should only work when the previous described left-wing party voting cue does not contradict it. Therefore, I expect that immigrant group voting is contingent on the endorsement of a CIO by a party left of the centre. This view is also supported by recent work of Zingher and Farrer, who find that in the UK and Australia the presence of a Labour ethnic minority candidate is associated with an increase in partisan support from ethnic minorities, but not the presence of centre-right minority candidates 
(Zingher \& Farrer, 2016). Since party-based voting cues are of greater relevance in partycentred electoral systems (Shugart et al., 2005), this relationship should be especially important in that category of electoral contexts.

H3: Immigrant-origin voters are more likely to support local CIOs' parties than other parties, conditional on CIOs' endorsement by parties left of the centre.

\section{Germany as a least-likely context for immigrant group voting}

For the purpose of testing these hypotheses, the present paper provides a case study of immigrant group voting in the 2013 German Bundestag election. In the past two decades, immigrant politics has been on the rise in Germany (e.g Schönwälder, 2012; Wüst, 2016). In 2013, approximately $20 \%$ of the residents and $9 \%$ of all eligible voters in Germany were of immigrant-origin, that is, born with foreign nationality or born to at least one parent with foreign nationality at birth (Bundeswahlleiter, 2013a). Consisting mainly of former "guest workers" and their descendants from south-eastern European countries or Turkey and of "ethnic Germans" from Central Eastern European countries, nowadays Germany accounts for $20 \%$ of the entire EU's immigrant population (OECD \& EU, 2015). Given higher fertility rates (Schönwälder, 2012) and more recent large-scale influxes of refugees (commonly touted the "refugee crisis") these numbers can be expected to grow even further in the future. Previous research in Germany shows that parties have responded to these developments by increasing their efforts to appeal to this new and growing segment of the electorate, indicated by an increasing salience of immigrant-related content in party manifestos and by increasing numbers of CIOs at all political levels (Fonseca, 2011; Schönwälder, 2012; Wüst, 2016).

However, an as of yet under-researched topic in German-specific research is whether immigrant-origin voters reward parties for their candidate offers. In Germany's mixedmember proportional (MMP) system, voters elect local representatives in 299 SMDs and 
simultaneously cast a vote for a closed party list in 16 multi-member districts which coincide geographically with the 16 federal states (PR tier). However, given that PR votes determine parties' seat shares in parliament due to a compensatory link between the two electoral tiers producing highly proportional election outcomes (Saalfeld, 2005), parties should mainly be interested in garnering $P R$ votes from the immigrant electorate. Consequently, the system is often considered as belonging to the family of PR systems (e.g. Plasser \& Plasser, 2002; Ruedin, 2013). Moser and Scheiner, for example, have described the German electoral system as "heavily party-oriented" with regard to citizens' voting behaviour due its strong linkage between its tiers and a long-consolidated party system (Moser \& Scheiner, 2005).

For these reasons, I consider Germany's PR tier as a party-centred electoral context, and thus a least-likely case of immigrant group voting from the perspective of the personal voting literature. As I argued in the previous section of this paper, nonetheless I expect CIOs' local presence to influence the party choices of voters of immigrant-origin. Therefore, I do not focus on the composition of the party list, i.e. the share of CIOs in a list (although I am going to control for this potential influence). In this respect, this paper's focus on the PR tier of Germany's MMP system also has a practical advantage: Germany facilitates the task of linking voters and CIOs in party lists unambiguously to the same geographical area, because most candidates run as double candidates, that is, in a party list and in a local constituency (Manow, 2015; Reiser, 2014). Simply put, the common practice of double candidacy facilitates studying whether immigrant-origin voters' electoral behaviour in the PR tier vary systematically with the local presence of CIOs, thus resembling previous research designs in more personalised electoral systems. 
Empirically, I analyse a merged dataset of candidate and voter data. The data on voters originates from the 2013 German Longitudinal Election Studies (GLES) ${ }^{2}$. The GLES data allows me to identify respondents of immigrant-origin from the question of whether they were born as German citizens or not, and by asking whether the respondent's mother and father were born in Germany or abroad. In order to avoid ending up with very few cases of immigrant voters, the merged file of the GLES pre- and post-election study is used. Since this paper is interested in PR vote choices, the dataset was reduced to all respondents who indicate that they either intend (pre-election study) or recall (post-election study) casting a PR vote for one of the main five party competitors (CDU/CSU, SPD, The Left, Greens, FDP), while nonvoters are ignored. Native respondents are kept in the dataset as a control group to which the voting behaviour of immigrant voters is compared to. Thus, the trimmed dataset includes immigrants (first generation), the direct descendants of immigrants (second generation) and native voters who indicate intention to vote in the 2013 elections for one of the main five parties. The final dataset contains 279 observations of voters of immigrant-origin and 2403 observations of native voters, overall 2682 observations.

The candidate dataset was compiled with the help of Parlamentwatch e.V., a registered German charity which presents detailed background information on all constituency candidates on its website (www.abgeordnetenwatch.de). The website administrators kindly provided the respective data, which then was complemented with official data from the Federal Electoral Commissioner (Bundeswahlleiter, 2013b) and sources described below. Candidates are coded as being of immigrant-origin if they were born either (a) abroad with foreign nationality at birth (first generation) or in Germany (b) with foreign nationality at birth or (c) with German nationality and at least one parent of foreign nationality at birth (second generation). Information on candidates' migratory backgrounds was taken from

\footnotetext{
${ }^{2}$ Please see Table A1 in the appendix file for a detailed overview and description of the GLES variables and their recoding used in the analysis.
} 
'Mediendienst Integration' (Mediendienst Integration, 2013) and other publicly available sources, such as personal and party websites, Wikipedia entries and interviews in newspaper. The candidate dataset contains overall 1493 observations of constituency candidates of which 96 were found to be of immigrant-origin.

Since both datasets contain an indicator variable for the constituency of voters and candidates, it is possible to merge the two dataset. However, given that the voter dataset provides observations for 252 of 299 constituencies, not all candidates could be matched. Table 1 gives an overview of the specific immigrant backgrounds of voters and of the candidates that remain in the merged dataset. Since the assignment of immigrant backgrounds to certain categories is predetermined in the GLES dataset, the candidate data was accommodated to match the GLES categorisation scheme shown in table 1. The second column shows the distribution of immigrant voters' origin in the dataset. Citizens of Polish-origin, closely followed by those descending from a country of the former Soviet Union, provide the largest groups $(2.4 \%)$ in the sample, while Turkish-origin respondents are in the third place with $1.3 \%$. These are also the three largest immigrant groups in the German population. The second column displays the distribution of the immigrant backgrounds of candidates which immigrant voters see on their ballot. Overall, every third immigrant voter $(34.1 \%)$ in the sample is confronted with a candidate of immigrant.

(Table 1 near here)

The dependent variable used in the present study refers to respondents' $P R$ vote choices between the main five competitor parties. In order to statistically investigate the variation in vote choices across respondents, it is crucial to note that a statistical model is necessary that is able to account for alternative specific and for individual specific variation at the same time, that is, factors that vary across vote choices and across respondents respectively. Basically, all variables measured at the candidate level are alternative specific variables. The independent 
variable $C I O$ is binary and captures whether a certain party vote option corresponds to a local CIO. In order to test the robustness of the analysis, the variable visible CIO was also coded to distinguish between candidates of German-origin and candidates whose immigrant-origin is easily recognisable based on their first name and surname or physical appearance. Unfortunately there is no information available regarding how "hard" candidates campaign for their parties. Thus, I assume that candidates' awareness of their local candidates reflects candidates' campaigning efforts. The intuition is that the harder candidates campaign in their constituencies the more likely it is that voters know their local candidates. In the GLES dataset, this information is provided by asking respondents for their recollection of local candidates' names and parties. Based on this information, I code the binary variable candidate awareness, if a candidate is able to tell by recollection the name of a candidate of a certain party in his/her constituency.

In addition to these variables, a couple of alternative-specific control variables are included. To account for the possibility that immigrant voters may respond to the CIO composition of party lists, the variable CIO list share measures the share of immigrant candidates among all list positions in a party list. Moreover, respondents' vote choice in the SMD tier (SMD vote) is included to control for the possibility that a voting decision in the PR tier is the result of a straight ticket, i.e. is due to supporting the candidates in the SMD tier. I further control for a number of variables associated with the socio-psychological, or "Michigan" model of voting (Campbell et al., 1980), which seems to provide the most explanatory power for the analysis of voting behaviour in the German context (Rudi \& Schoen, 2014). Thus, it is controlled for voters' party identification. Including this variable will also help to account for the possibility that immigrants' support for a CIO is simply an unobserved effect of immigrant voters' support for left parties which are also more likely to nominate CIOs (Bergh \& Bjorklund, 2011). The variable party identification covers whether a voter identifies with a certain party 
as well as the strength of this identification. Based on the GLES dataset, a value of 0 denotes that a voter does not identify with a candidate's party, and values between 1 and 5 indicate a weak to very strong identification with that party.

A second set of control variables is measured individual specific. Rile self-placement measures the self-placement of a voter on a scale from 1 (most left) to 11 (most right). To account for the effect of candidates for chancellor, the variable chancellor preference captures whether a voter prefers the CDU/CSU (Angela Merkel) or SPD top candidate (Peer Steinbrück) or neither. Furthermore, since Wüst (2004) shows that immigrants descending from post-soviet countries of emigration tend to support the CDU/CSU while most other immigrant groups support parties of the left in Germany, it is controlled for the two largest immigrant groups in the dataset, that is, voters descending from Poland or a country of the former USSR, with remaining immigrant respondents contained in a reference category. First generation, captures in addition to that whether a voter has immigrated herself $(=1)$ or whether s/he was born to immigrant parents $(=0)$. Post-election survey accounts for whether a respondent was part of the GLES 2013 post-election study $(=1)$, or of the pre-election study $(=0)$. Finally, with system knowledge it is controlled for whether or not the respondent understands the consequences of his two vote choices. If the respondent can tell that the party list vote determines a party's overall seat share, the variable takes a value of 1 and 0 otherwise.

(Table 2 near here)

In order to be transparent about a possible bias of immigrants' PR votes, party identification and the selection of CIOs towards parties left of the centre, Table 2 presents how these partyrelated variables are distributed in the dataset. As can be seen in the first row, PR votes of immigrant respondents distribute rather evenly between right (CDU/CSU and FDP) and leftleaning parties (SPD, Greens, The Left), resembling similar patterns in the population of 
German-origin voters (second row). Neither the partisanship of immigrant respondents is biased towards left-leaning parties as shown in the third row, also resembling patterns in the population of German-origin voters shown in the fourth row. However, immigrant and native voters are both more likely to see CIOs of left-wing than of right-wing parties (fifth and sixth row). This picture, however, coincides with the distribution of constituency CIOs across all parties in the candidate dataset (seventh row). Therefore, it is rather a real world fact that most immigrant candidates run for parties of the political left than a bias in the used data.

\section{Multivariate analysis}

I employ alternative-specific multinomial logit models, given that the main independent variable of interest $\mathrm{CIO}$ varies across party choices, while several control variables vary across voters. Formally, this model uses the formula

$$
\operatorname{Pr}\left(y_{i}=m \mid x_{i}, z_{i}\right)=\frac{\exp \left(z_{i m} \gamma+x_{i} \beta_{m}\right)}{\sum_{j=1}^{J} \exp \left(z_{i j} \gamma+x_{i} \beta_{j}\right)}, \quad \text { with } \beta_{1}=0
$$

where the probability of observing outcome $\mathrm{m}$ depends on alternative specific variables $\mathrm{z}_{\mathrm{im}}$ as well as individual specific variabels $\mathrm{x}_{\mathrm{i}}$. While the effect of the alternative specific variables is contained in $\gamma$, the coefficients for the effects of the individual specific variables on outcome $\mathrm{m}$ are contained in $\beta$ relative to the baseline category (Long $\&$ Freese, 2014, pp. 460-61). In order to fit this statistical model, however, the data needs to be rearranged in a way that each respondent appears five times (in five rows) in the dataset, that is, one time for each party choice. Alternative-specific variables can thus vary across these five alternatives. Individual specific variables, by contrast, take values that are the same across the five alternatives.

The empirical strategy is to fit models separately for immigrant and native voters in order to examine whether associations found in the sample of immigrants are actually either immigrant specific or resemble patterns in the population of native voters. Table 3 shows the 
alternative-specific components ${ }^{3}$ of four alterative-specific multinomial logit models supposed to test this paper's hypotheses.

(Table 3 here)

In models 1 and 2 it can be observed that respondents' awareness of local candidates, their party identification and SMD votes influence immigrants' and natives' PR votes significantly and in expected ways. However, controlling for these and other variables in the individual specific equations immigrants' likelihood of supporting a party is estimated to be positively and significantly affected by the presence of a local CIO from that party, while natives' party support remains unaffected. The share of CIOs in a party lists, by contrast, is not estimated to have a significant effect in either of the first two models. These results coincide with H1.

In models 3 and 4, I also include the interaction effect between CIO and candidate awareness for the purpose of testing $\mathrm{H} 2$, that is, whether the effect of the local presence of a CIO on immigrant-origin voters' support for a party list is actually dependent on a voters' awareness of the candidates (as a proxy of candidates' local campaigning efforts). As can be seen, the hypothesised relationship holds. When the interaction is added in the model for immigrants (model 3), the coefficient of the CIO variable turns out to be statistically insignificant, while the interaction term turns out to be positive and highly significant. In the control group of natives, by comparison, neither CIO nor its interaction with candidate awareness shows significant effects.

(Figure 1 here)

How do these effects vary by party choice? For this purpose, Figure 1 provides the marginal effects of voters' awareness of a CIO on their specific party support, based on models 3 and 4

\footnotetext{
${ }^{3}$ Since the complete model outputs are rather huge and thus unwieldy for presentation in the paper, the full regression tables are supplied in Table A2 in the appendix file to this paper.
} 
of Table 3. That is, the illustration visualises the simulation of how the likelihood of supporting any of the five parties changes when voters are confronted with a CIO as compared to when they are confronted with a native candidate from a specific party. ${ }^{4}$ Given that the relationship is contingent on candidate awareness, this variable is held at one in the estimations. Starting with the marginal effects in the group of immigrant voters, it can be seen that the effect of CIO varies strongly by party. The marginal effects for the two right-wing parties, FDP and CDU/CSU, are shown in the top and bottom plots. They suggest that the nomination of a CIO by one of these parties increases candidate-aware immigrant voters' likelihood of supporting the party by 0.10 and 0.13 respectively. However, these effects are not estimated to be statistically significant. The picture changes, however, for the three parties on the left. When immigrant voters are aware of a CIO from the Greens (The Left, SPD), the likelihood of supporting this party increases by $0.62(0.66,0.41)$. Moreover, the plots suggest a trade-off between the three left-wing parties, that is, immigrant-origin citizens' voting decisions are affected by the presence of a left-wing CIO in that the party of the CIO becomes more likely to be voted for to the extent that the other two left-wing parties become less likely to be voted for. In the control group of native voters, by contrast, the picture could not be more different. Irrespective of party label, native voters' PR vote choices are not found to be affected at all by the presence of a CIO when they are aware of the candidate.

The results are robust to a variety of different model specifications, which can be inspected in tables A3 to A5 in the Appendix. Table A3 shows that the same results can be replicated when the data is not weighted in the statistical estimation to compensate for an oversampling of East German regions and to make the data more representative of the socio-structural outlook of the German voting age population. Table A4 presents additional models with the variable CIO being exchanged for visible CIO. And finally, table A5 shows the results of two

\footnotetext{
${ }^{4}$ Following suggestions of Hanmer and Kalkan, the marginal effects are based on the empirical observed values of remaining variables in model 3 (Hanmer \& Kalkan, 2013).
} 
alternative-specific mixed multinomial logit models to check whether the CIO effect suffers from a potential violation of the independence of irrelevant alternatives assumption (IIAA). By estimating random coefficients for the independent variables of interest and fixed effects for remaining coefficients, the alternative-specific mixed logit approach allows for correlations across alternatives in the random part of the estimation, thus relaxing the IIAA and providing a robustness check for the results of standard alternative-specific logit models (for details see Ben-Akiva et al., 2001; Cameron \& Trivedi, 2005, pp. 513-16).

\section{Discussion and Conclusions}

This paper engages with the research puzzle of immigrant group voting under party-centred electoral rules. From the perspective of the personal voting literature (Carey \& Shugart, 1995; Shugart et al., 2005), it appears questionable whether parties can utilise CIOs to attract votes from the immigrant-origin electorate in such systems. I argue in this paper that immigrant group voting is a decisive factor under party-centred electoral rules. In my argumentation, parties have incentives to distribute list CIOs geographically as a campaigning resource in order to tap into immigrant-origin voter markets. Because CIOs are typically locally connected, for example as local councillors or party officials, they constitute a local voting cue to immigrant-origin voters. Provided that these candidates are perceived to better represent disadvantaged groups' interests than native candidates, immigrant-origin voters should be more likely to support the parties of local CIOs than those of other parties.

For a test of this argument, I study immigrant-origin citizens' voting behaviour in the 2013 German Bundestag election based on a merged dataset of survey and candidate data. The main findings suggest that immigrant group voting is considerable in Germany: immigrant voters' PR votes are strongly affected by the local presence of CIOs. The proposed argument for this link, that is, candidates' local visibility being the main driver of this relationship, is further corroborated by the finding that the statistical association is mainly due to voter's 
awareness of their local CIOs. Finally, my analysis also suggests that this kind of party competition for immigrant-origin voters is mainly a game between parties of the left, in which the win of one party means the loss of another one. Crucially, all these relationships were found to be immigrant-specific, that is, the reported effects could not be replicated in the control group of native voters.

It is important to note that the present study has limitations, though. One is that the analysis lacks the statistical power to isolate the relationship between voters and candidates of same descent from that between voters and candidates belonging to different national groups. However, in line with Zingher and Farrer it can be argued that this lack of precision should decrease the odds of verifying the immigrant group voting hypothesis, thus providing for an even tougher test of the argument (Zingher \& Farrer, 2016). The finding of a significant relationship nonetheless provides therefore strong evidence in favour of immigrant group voting and suggests that "linked fate" perceptions associated with the presence of a CIO may surpass ethnic and/or national boundaries with regard to voting behaviour.

Doubts may also be expressed as to whether these results are driven by the case selection of Germany's MMP system. Indeed, the simultaneous existence of an electoral tier operating in geographical constituencies and the common double candidacy practice constitute a unique feature of MMP as compared to "pure" closed-list PR systems. However, I argue that this finding should apply to the latter type of system nonetheless, because the two systems show striking similarities with regards to key aspects of the proposed argument. First, both systems ensure a high level of proportional representation, due to which they provide similar voteseeking incentives for parties as each additional vote has the potential to increase a party's seat shares. Second, locally connected list candidates are not unique to Germany, given the common empirical patter of list candidates holding simultaneously local government or party offices in a number of closed-list PR systems. Since immigrant-origin citizens tend to cluster 
geographically, it is therefore plausible to assume that in both types of systems parties have incentives to exploit their CIOs' local attachments to create vote returns from the immigrantorigin electorate. Nevertheless, future research is needed to examine the generalisability of the immigrant group voting link in a variety of party-centred electoral systems, for example in the Netherlands or Israel, where two of the most proportional closed-list systems are combined with the requirement for candidates to retain local attachments (Hazan, 1999; Leijenaar \& Niemöller, 1997).

This paper makes a major contribution to previous literature nonetheless. It extends previous research (Barreto, 2007; Bergh \& Bjorklund, 2011; Landa et al., 1995; McConnaughy et al., 2010; Teney et al., 2010; Zingher \& Farrer, 2016), by presenting the first case study that supports the hypothesis of immigrant group voting in a least-likely party-centred electoral environment. Thereby, it contributes to this literature by raising doubts about the moderating influence of electoral systems. Least-likely case studies, are essential for the accumulation of knowledge, because they make it harder to corroborate deductively derived hypotheses and help to rule out rival hypothesis, thus having the potential to increase our confidence in the validity of the hypotheses if supported (Gerring \& Seawright, 2007). As such, this case study substantiates the frequently made assumption that parties in party-centred systems of proportional representation have vote-seeking incentives to provide for more descriptive representation of immigrant-origin citizens in their party lists. Moreover, a secondary finding of the presented analysis may also point to a possible advantage of party-centred as compared to candidate-centred electoral systems. The non-finding of an effect of CIOs in the control group of native voters, a finding that reiterates previous research in Germany (Street, 2014), stands in contrast to the repeatedly found electoral penalty for ethnic minority candidates in the UK's first-past-the-post system (e.g. Fisher et al., 2015; Stegmaier et al., 2013). A possible explanation may be that CIOs face less discrimination in more party-centred electoral 
systems, because they provide for weaker personal voting incentives, while at the same time ensuring that locally connected CIOs remain visible to immigrant-origin voters as a result of the proposed argument. At this point, however, this intuition is largely speculative but may be an interesting research question for succeeding research. 
References

Alba, R. \& Foner, N. (2015). Strangers No More: Immigration and the Challenges of Integration in North America and Western Europe. Princeton: Princeton University Press.

Banducci, S., Donovan, T. \& Karp, J. (2004). Minority Representation, Empowerment and Participation. Journal of Politics 66(2): 534-556.

Barreto, M.A. (2007). ¡S1 SePuede! Latino Candidates and theMobilization of LatinoVoters. American Political Science Review 101(3): 425-441.

Ben-Akiva, M., Bolduc, D. \& Walker, J. (2001). Specification, identification, and estimation of the logit kernel (or continuous mixed logit) model. Berkeley. Retrieved from http://eml.berkeley.edu/reprints/misc/multinomial2.pdf

Bergh, J. \& Bjorklund, T. (2011). The Revival of Group Voting: Explaining the Voting Preferences of Immigrants in Norway. Political Studies 59(2): 308-327.

Bird, K., Saalfeld, T. \& Wüst, A.M. (2011a). Ethnic Diversity, Political Participation and Representation: a Theoretical Framework. In K. Bird, T. Saalfeld \& A. M. Wüst (eds.), The Political Representation of Immigrants and Minorities. Voters, Parties and Parliaments in Liberal Democracies. London and New York: Routledge.

Bird, K., Saalfeld, T. \& Wüst, A.M. (2011b). The Political Representation of Immigrants and Minorities. Voters, Parties and Parliaments in Liberal Democracies. London and New York: Routledge.

Bloemraad, I. \& Schönwälder, K. (2013). Immigrant and Ethnic Minority Representation in Europe: Conceptual Challenges and Theoretical Approaches. West European Politics 36(3): 564-579.

Bundeswahlleiter. (2013a). 5,8 Millionen Deutsche mit Migrationshintergrund sind wahlberechtigt. Retrieved 9 March 2016, from https://www.bundeswahlleiter.de/de/bundestagswahlen/BTW_BUND_13/presse/W1301 3_Wahlberechtigte_Migrationshintergrund.html

Bundeswahlleiter. (2013b). Wahl zum 18. Deutschen Bundestag am 22. September 2013. Sonderheft: Die Wahlbewerber für die Wahl zum 18. Deutschen Bundestag 2013. 
Wiesbaden.

Cameron, A.C. \& Trivedi, P.K. (2005). Microeconometrics: Methods and Applications. Cambridge: Cambridge University Press.

Campbell, A., Converse, P.E., Miller, W.E. \& Stokes, D.E. (1980). The American Voter. Chicago: University of Chicago Press.

Carey, J.M. \& Shugart, M.S. (1995). Incentives to cultivate a personal vote: A rank ordering of electoral formulas. Electoral Studies 14(4): 417-439.

Dancygier, R.M. \& Saunders, E.N. (2006). A New Electorate? Comparing Preferences and Partisanship between Immigrants and Natives. American Journal of Political Science 50(4): 962-981.

Fernandes, J.M., Leston-Bandeira, C. \& Schwemmer, C. (2017). Election proximity and representation focus in party-constrained environments. Party Politics (July 2016): 135406881768995.

Fisher, S.D., Heath, A.F., Sanders, D. \& Sobolewska, M. (2015). Candidate Ethnicity and Vote Choice in Britain. British Journal of Political Science 45(4): 883-905.

Fonseca, S.C. da. (2011). New citizens- new candidates? Candidate selection and the mobilization of immigrant voters in German elections. In K. Bird, T. Saalfeld \& A. M. Wüst (eds.), The Political Representation of Immigrants and Minorities. London and New York: Routledge.

Gerring, J. \& Seawright, J. (2007). Techniques for Choosing Cases. In J. Gerring (ed.), Case Study Research. Principles and Practices. Cambridge: Cambridge University Press.

Hanmer, M.J. \& Kalkan, K.O. (2013). Behind the Curve: Clarifying the Best Approach to Calculating Predicted Probabilities and Marginal Effects from Limited Dependent Variable Models. American Journal of Political Science 57(1): 263-277.

Hazan, R.Y. (1999). Constituency interests without constituencies: the geographical impact of candidate selection on party organization and legislative behavior in the 14th Israeli Knesset, 1996-99. Political Geography 18: 791-811.

Kittilson, M.C. \& Tate, K. (2005). Political parties, minorities and elected office : comparing opportunities for inclusion in the United States and Britain. In C. Wolbrecht \& R. E. 
Hero (eds.), ThePolitics of Democratic Inclusion. Philadelphia: Temple University Press.

Landa, J., Copeland, M. \& Grofman, B. (1995). Ethnic voting patterns: a case study of Metropolitan Toronto. Political Geography 14(5): 435-449.

Latner, M. \& McGann, A. (2005). Geographical representation under proportional representation: The cases of Israel and the Netherlands. Electoral Studies 24(4): 709734.

Laurence, J. \& Maxwell, R. (2012). Political Parties and Diversity in Western Europe. In T. Givens \& R. Maxwell (eds.), Immigrant Politics: Race and Representation in Western Europe. Boulder: Lynne Rienner Publishers.

Leijenaar, M. \& Niemöller, K. (1997). The Netherlands. In P. Norris (ed.), Passages to power. Legislative recruitment in advanced democracies. Cambridge: Cambridge University Press.

Long, J.S. \& Freese, J. (2014). Regression Models for Categorical Dependent Variables Using Stata (3rd ed.). Texas: Stata Press.

Manow, P. (2015). Mixed Rules, Mixed Strategies. Candidates and Parties in Germany's Electoral System. Colchester: ECPR Press.

McConnaughy, C.M., White, I.K., Leal, D.L. \& Casellas, J.P. (2010). A Latino on the Ballot: Explaining Coethnic Voting Among Latinos and the Response of White Americans. The Journal of Politics 72(4): 1199-1211.

McDermott, M.L. (1998). Race and Gender Cues in Low-Information Elections. Political Research Quarterly 51(4): 895-918.

Mediendienst Integration. (2013). Bundestagskandidaten mit Migrationshintergrund. Retrieved 9 March 2016, from https://mediendienstintegration.de/fileadmin/Dateien/Bundestagskandidaten_MDI.pdf

Messina, A.M. (2007). The Logics and Politics of Post-WWII Migration to Western Europe. Cambridge: Cambridge University Press.

Moser, R.G. \& Scheiner, E. (2005). Strategic Ticket Splitting and the Personal Vote in Mixed-Member Electoral Systems. Legislative Studies Quarterly 30(2): 259-276. 
OECD \& EU. (2015). Indicators of Immigrant Integration 2015: Settling In. Paris: OECD Publishing.

Plasser, F. \& Plasser, G. (2002). Global Political Campaigning: A Worldwide Analysis of Campaign Professionals and their Practices. Westport, CT: Praeger.

Rattinger, H., Roßteutscher, S., Schmidt-Beck, R., Weßels, B. \& Wolf, C. (2014). Vor- und Nachwahl-Querschnitt (GLES 2013). ZA5702 Datenfile Version 2.0.0. Köln: GESIS Datenarchiv.

Reiser, M. (2014). The universe of group representation in Germany: Analysing formal and informal party rules and quotas in the process of candidate selection. International Political Science Review 35(1): 55-66.

Rudi, T. \& Schoen, H. (2014). Ein Vergleich von Theorien zur Erklärung von Wählerverhalten. In J. W. Falter \& H. Schoen (eds.), Handbuch Wahlforschung. Wiesbaden: Springer VS.

Ruedin, D. (2013). Why Aren't They There? The Political Representation of Women, Ethnic Groups and Issue Positions in Legislatures. Colchester: ECPR Press.

Russo, F. (2011). The Constituency as a Focus of Representation: Studying the Italian Case through the Analysis of Parliamentary Questions. The Journal of Legislative Studies 17(3): 290-301.

Saalfeld, T. (2005). Germany: Stability and Strategy in a Mixed-Member Proportional System. In G. Michael \& P. Mitchell (eds.), The Politics of Electoral Systems. Oxford and New York: Oxford University Press.

Sanders, D., Heath, A.F., Fisher, S.D. \& Sobolewska, M. (2014). The Calculus of Ethnic Minority Voting in Britain. Political Studies 62: 230-51.

Schönwälder, K. (2012). Cautious Steps: Minority Representation in Germany. In T. E. Givens \& R. Maxwell (eds.), Immigrant Politics: Race and Representation in Western Europe. Boulder: Lynne Rienner Publishers.

Shugart, M., Valdini, M.E. \& Suominen, K. (2005). Looking for locals: Voter information demands and personal vote-earning attributes of legislators under proportional representation. American Journal of Political Science. 
Sobolewska, M., Fieldhouse, E. \& Cutts, D. (2013). Taking Minorities for Granted? Ethnic Density. Party Campaigning and Targeting Minority Voters in 2010 British General Elections. Parliamentary Affairs 66(2): 329-344.

Stegmaier, M., Lewis-Beck, M.S. \& Smets, K. (2013). Standing for Parliament: Do Black, Asian and Minority Ethnic Candidates Pay Extra? Parliamentary Affairs 66(2): 268-85.

Street, A. (2014). Representation Despite Discrimination: Minority Candidates in Germany. Political Research Quarterly 67(2): 374-385.

Strijbis, O. (2014). Migration Background and Voting Behavior in Switzerland: A SocioPsychological Explanation. Swiss Political Science Review 20(4): 612-631.

Strömbäck, J. (2009). Selective professionalisation of political campaigning: A test of the party-centred theory of professionalised campaigning in the context of the 2006 Swedish election. Political Studies 57(1): 95-116.

Tajfel, H. \& Turner, J.C. (1986). The Social Identity Theory of Intergroup Behavior. In S. Worchel \& W. G. Austin (eds.), Psychology of Intergroup Relations. Chicago: NelsonHall.

Teney, C., Jacobs, D., Rea, A. \& Delwit, P. (2010). Ethnic voting in Brussels: Voting patterns among ethnic minorities in Brussels (Belgium) during the 2006 local elections. Acta Politica 45(3): 273-97.

Wessels, B. (1997). Germany. In P. Norris (ed.), Passages to power. Legislative recruitment in advanced democracies. Cambridge: Cambridge University Press.

Wüst, A.M. (2004). Naturalised citizens as voters: behaviour and impact. German Politics 13(2): 341-359.

Wüst, A.M. (2016). Incorporation beyond Cleavages? Parties, Candidates and Germany's Immigrant-Origin Electorate. German Politics 25(2): 414-432.

Zingher, J.N. \& Farrer, B. (2016). The electoral effects of the descriptive representation of ethnic minority groups in Australia and the UK. Party Politics 22(6): 691-704. 
Date: January 142018

Word count (including Tables, Figures and Captions): 7,509 
Table 1. Voters' and candidates' immigrant backgrounds in the dataset

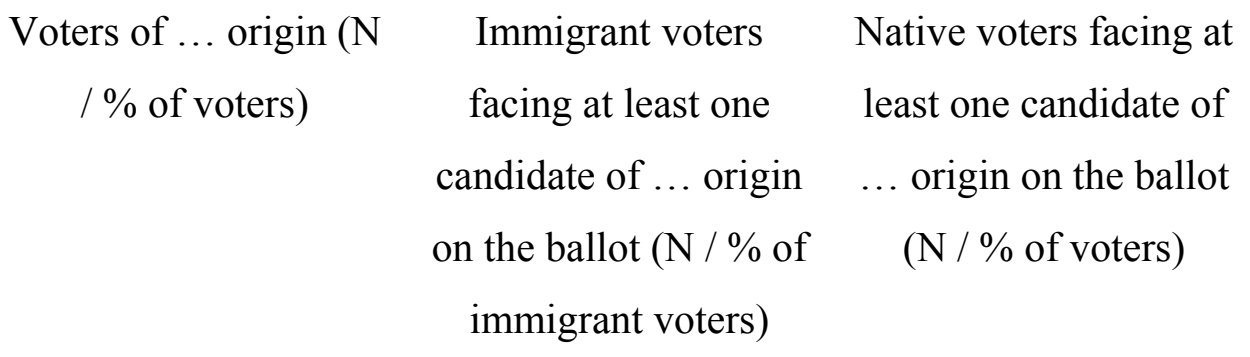

\begin{tabular}{lccc}
\hline German & $2403 / 89.6 \%$ & $279 / 100 \%$ & $2403 / 100 \%$ \\
\hline Any immigrant & $279 / 10.4 \%$ & $95 / 34.1 \%$ & $615 / 25.6 \%$ \\
\hline Turkish & $36 / 1.3 \%$ & $41 / 14.7 \%$ & $259 / 10.8 \%$ \\
\hline Italian & $10 / 0.4 \%$ & 0 & $7 / 0.3 \%$ \\
\hline Polish & $65 / 2.4 \%$ & 0 & $12 / 0.5 \%$ \\
\hline Former USSR & $64 / 2.4 \%$ & $6 / 2.2 \%$ & $43 / 1.8 \%$ \\
\hline Greek & $4 / 0.1 \%$ & $2 / 0.7 \%$ & $21 / 0.9 \%$ \\
\hline Former & $13 / 0.5 \%$ & $3 / 1.1 \%$ & $26 / 1.1 \%$ \\
Yugoslavia & $4 / 0.1 \%$ & $2 / 0.7 \%$ & $21 / 0.9 \%$ \\
\hline Austrian & $31 / 1.2 \%$ & $9 / 3.2 \%$ & 0 \\
\hline Czech/ Slovak & $2 />0.1 \%$ & 0 & $55 / 2.3 \%$ \\
\hline Scandinavian & $3 / 0.1 \%$ & $6 / 2.2 \%$ & $10 / 0.4 \%$ \\
\hline Belgian/ Dutch & $2 />0.1 \%$ & $2 / 0.7 \%$ & 0 \\
\hline French & $1 />0.1 \%$ & 0 & $233 / 9.7 \%$ \\
\hline US & $44 / 1.6 \%$ & $33 / 11.8 \%$ & \\
\hline Other & & & 0 \\
\hline
\end{tabular}


Table 2. Distribution of party-related variables in the used dataset

CDU/CSU FDP SPD Green The Left no party

\begin{tabular}{|c|c|c|c|c|c|c|c|}
\hline Immigrants' PR & $\mathrm{N}$ & 122 & 16 & 66 & 46 & 29 & - \\
\hline votes for... ${ }^{a}$ & $\%$ & 43.7 & 5.7 & 23.7 & 16.5 & 10.4 & \\
\hline Natives' PR votes & $\mathrm{N}$ & 1,015 & 101 & 747 & 247 & 293 & - \\
\hline for... ${ }^{a}$ & $\%$ & 42.24 & 4.2 & 31.1 & 10.3 & 12.2 & \\
\hline Immigrants & $\mathrm{N}$ & 106 & 5 & 64 & 33 & 17 & 54 \\
\hline identifying with... ${ }^{a}$ & $\%$ & 38.0 & 1.8 & 22.9 & 11.8 & 6.1 & 19.4 \\
\hline Natives identifying & $\mathrm{N}$ & 882 & 47 & 650 & 170 & 219 & 435 \\
\hline with... ${ }^{\mathrm{a}}$ & $\%$ & 36.7 & 2.0 & 27.0 & 7.1 & 9.1 & 18.1 \\
\hline Immigrants who see & $\mathrm{N}$ & 5 & 10 & 18 & 34 & 31 & - \\
\hline a CIO from ... & $\%$ & 1.8 & 3.6 & 6.5 & 12.2 & 11.1 & \\
\hline Natives who see a & $\mathrm{N}$ & 66 & 39 & 162 & 187 & 212 & - \\
\hline $\mathrm{CIO}$ from $\ldots{ }^{\mathrm{ab}}$ & $\%$ & 2.7 & 1.6 & 6.7 & 7.8 & 8.8 & \\
\hline Total no. of CIO & $\mathrm{N}$ & 9 & 9 & 20 & 26 & 32 & - \\
\hline running for... ${ }^{c}$ & $\%$ & 0.6 & 0.6 & 1.3 & 1.7 & 2.1 & \\
\hline
\end{tabular}

Note: based on the data described in the text; ${ }^{a}$ based on the merged data file; ${ }^{b}$ voters may see more than one candidate of immigrant-origin; ${ }^{\mathrm{c}}$ based on the candidate data file, percentages based on all candidate observation 
Table 3: Alternative-specific determinants of immigrants' and natives' PR votes

\begin{tabular}{|c|c|c|c|c|}
\hline & $\begin{array}{c}\text { Model 1: } \\
\text { Immigrants' } \\
\text { PR votes }\end{array}$ & $\begin{array}{l}\text { Model 2: } \\
\text { Natives' } \\
\text { PR votes }\end{array}$ & $\begin{array}{c}\text { Model 3: } \\
\text { Immigrants' } \\
\text { PR votes }\end{array}$ & $\begin{array}{l}\text { Model 4: } \\
\text { Natives' } \\
\text { PR votes }\end{array}$ \\
\hline \multicolumn{5}{|c|}{ Alternative-specific equation: } \\
\hline $\mathrm{CIO}$ & $\begin{array}{l}1.51 * * \\
(0.49)\end{array}$ & $\begin{array}{l}-0.22 \\
(0.32)\end{array}$ & $\begin{array}{c}0.31 \\
(0.42)\end{array}$ & $\begin{array}{l}-0.42 \\
(0.39)\end{array}$ \\
\hline Candidate awareness & $\begin{array}{l}1.73 * * \\
(0.54)\end{array}$ & $\begin{array}{c}0.86^{* * * *} \\
(0.15)\end{array}$ & $\begin{array}{l}1.44^{*} \\
(0.62)\end{array}$ & $\begin{array}{c}0.84 * * * \\
(0.14)\end{array}$ \\
\hline CIO \# awareness & & & $\begin{array}{c}2.91 * * * \\
(0.87)\end{array}$ & $\begin{array}{c}0.39 \\
(0.62)\end{array}$ \\
\hline Party identification & $\begin{array}{c}0.75 * * * \\
(0.16)\end{array}$ & $\begin{array}{c}0.64 * * * \\
(0.05)\end{array}$ & $\begin{array}{c}0.77 * * * \\
(0.17)\end{array}$ & $\begin{array}{c}0.64 * * * \\
(0.05)\end{array}$ \\
\hline SMD vote & $\begin{array}{l}0.64+ \\
(0.34)\end{array}$ & $\begin{array}{c}1.39 * * * \\
(0.12)\end{array}$ & $\begin{array}{l}0.62+ \\
(0.38)\end{array}$ & $\begin{array}{c}1.39 * * * \\
(0.12)\end{array}$ \\
\hline CIO list share & $\begin{array}{c}0.97 \\
(1.59)\end{array}$ & $\begin{array}{l}-0.35 \\
(0.50)\end{array}$ & $\begin{array}{c}0.97 \\
(1.70)\end{array}$ & $\begin{array}{l}-0.38 \\
(0.52)\end{array}$ \\
\hline \multicolumn{5}{|c|}{ Individual-specific equations omitted (see appendix file) } \\
\hline $\begin{array}{l}\mathrm{N} \text { (alternatives) } \\
\mathrm{N} \text { (cases) } \\
\mathrm{Bic}\end{array}$ & $\begin{array}{c}1235 \\
247 \\
641.80\end{array}$ & $\begin{array}{c}10930 \\
2154 \\
2510.60\end{array}$ & $\begin{array}{c}1235 \\
247 \\
632.58\end{array}$ & $\begin{array}{c}10930 \\
2154 \\
2518.85\end{array}$ \\
\hline
\end{tabular}

Note: $+\mathrm{p}<0.10,{ }^{*} \mathrm{p}<0.05,{ }^{* *} \mathrm{p}<0.01,{ }^{* * *} \mathrm{p}<0.001$; robust standard errors, clustered on constituencies, are shown in parentheses; models estimated on weighted data to compensate for an oversampling of East German regions and to make the data more representative of the socio-structural outlook of the German voting age population (weight variable in the GLES 2013 dataset: $\mathrm{w}$ ipfges_2); ${ }^{\mathrm{a}}$ reference category is remaining immigrant groups; ${ }^{b}$ reference group is Merkel 
Voters of immigrant origin

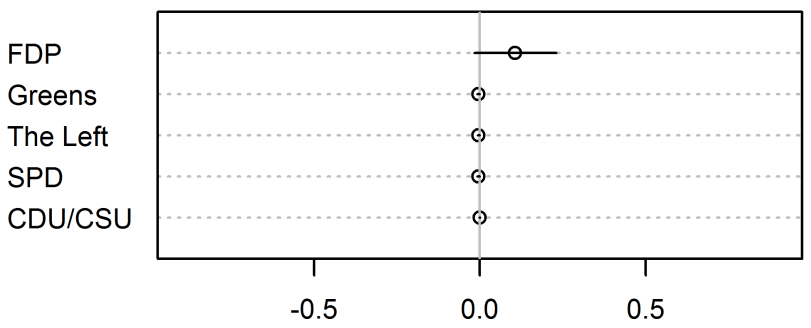

ME of $\mathrm{CIO}$ from FDP, holding candidate awareness at 1

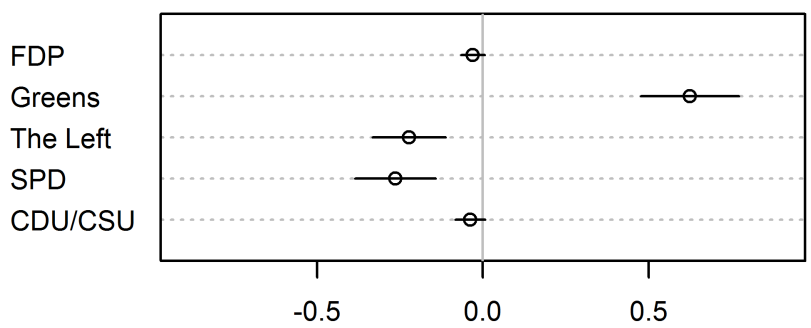

$\mathrm{ME}$ of $\mathrm{ClO}$ from Green Party, holding candidate awareness at 1

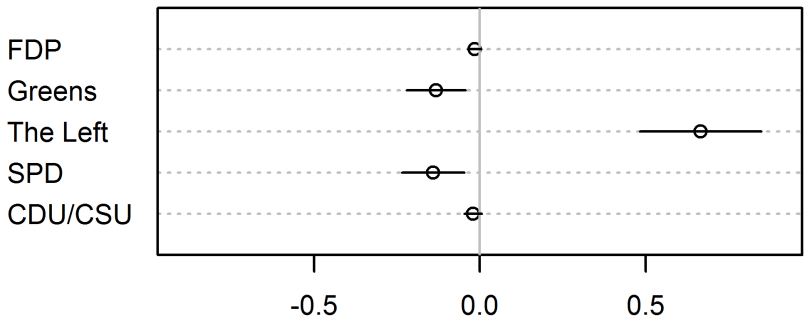

ME of $\mathrm{ClO}$ from The Left, holding candidate awareness at 1

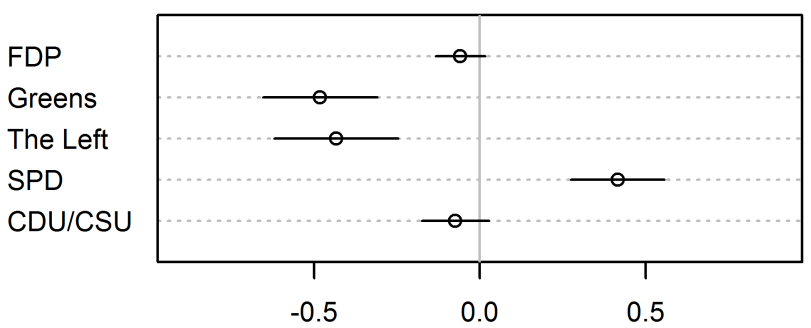

ME of $\mathrm{CIO}$ from SPD, holding candidate awareness at 1

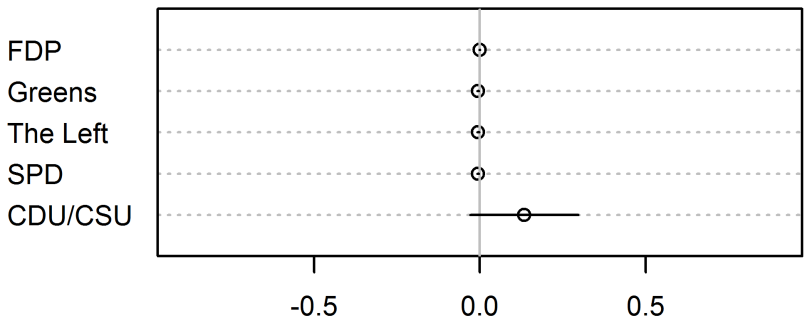

$\mathrm{ME}$ of $\mathrm{CIO}$ from $\mathrm{CDU} / \mathrm{CSU}$, holding candidate awareness at 1

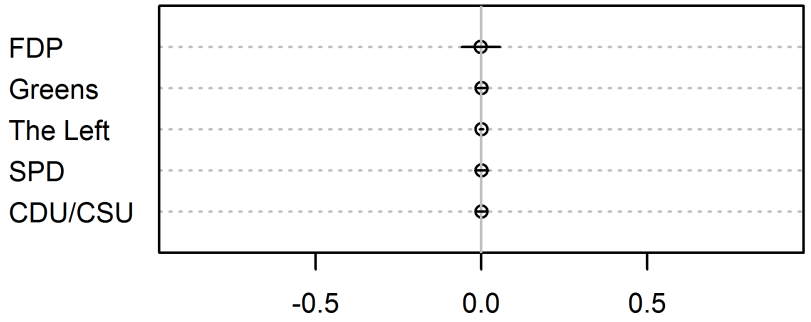

ME of $\mathrm{CIO}$ from FDP, holding candidate awareness at 1

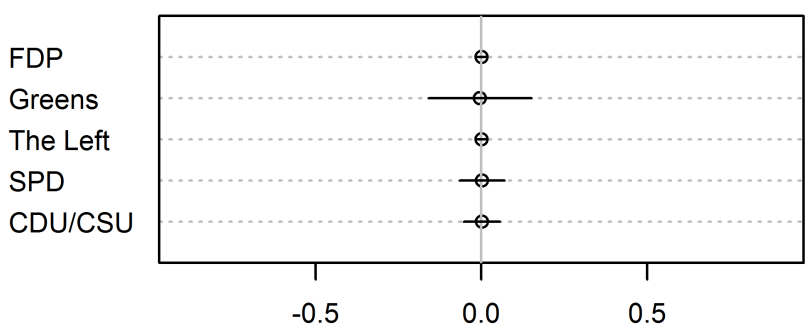

$\mathrm{ME}$ of $\mathrm{ClO}$ from Green Party, holding candidate awareness at 1

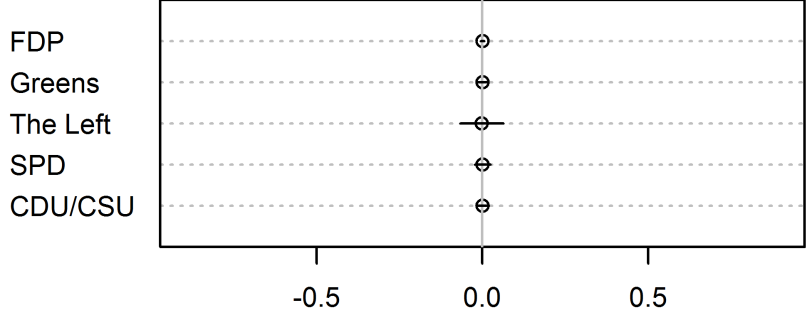

ME of CIO from The Left, holding candidate awareness at 1

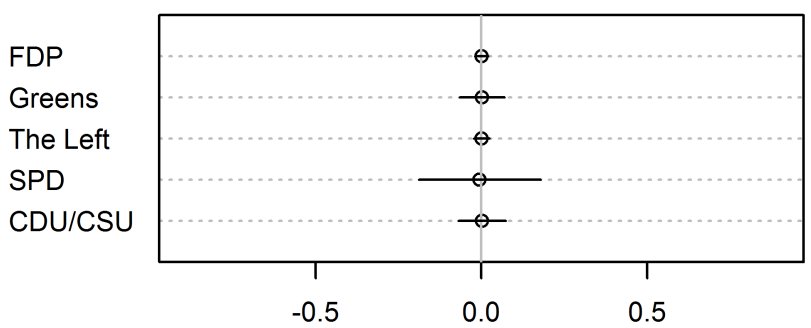

ME of CIO from SPD, holding candidate awareness at 1

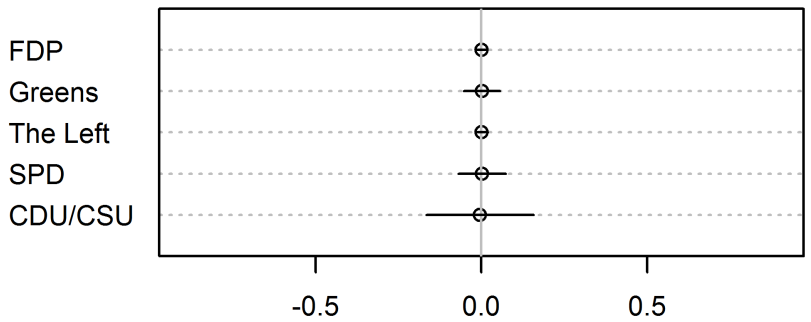

$\mathrm{ME}$ of $\mathrm{CIO}$ from $\mathrm{CDU} / \mathrm{CSU}$, holding candidate awareness at 1 
Appendix file to paper

"Immigrant group voting despite party-centred electoral rules: evidence from Germany" 
Table A1: Variables in the GLES 2013 dataset and recoding

\begin{tabular}{|c|c|c|}
\hline $\begin{array}{l}\text { Original variable name(s) in } \\
\text { dataset }\end{array}$ & $\begin{array}{l}\text { Questionnaire wording (own } \\
\text { translation) }\end{array}$ & Recoding/ Usage \\
\hline $\begin{array}{l}\text { v11ba (Party vote: pre-election } \\
\text { survey) } \\
\text { n11ba (Party vote: post-election } \\
\text { survey) }\end{array}$ & $\begin{array}{l}\text { Example pre-election study: } \\
\text { "In the Bundestag election you } \\
\text { can cast two votes. The first } \\
\text { vote for a candidate in your } \\
\text { electoral district and a second } \\
\text { vote for a party. Here is a ballot } \\
\text { specimen similarly to the one } \\
\text { you will be provided with forthe } \\
\text { election. How are you going to } \\
\text { vote?" } \\
\text { (21) CDU/CSU (Christlich } \\
\text { Demokratische Union/Christich- } \\
\text { Soziale Union) } \\
\text { (22) SPD (Sozialdemokratische } \\
\text { Partei Deutschlands) } \\
\text { (23) FDP (Freie Demokratische } \\
\text { Partei) } \\
\text { (24) DIE LINKE (DIE LINKE) } \\
\text { (25) GRÜNE (BÜNDNIS } \\
\text { 90/DIE GRÜNEN) } \\
\text { (26) PIRATEN (Piratenpartei } \\
\text { Deutschland) } \\
\text { (27) NPD } \\
\text { (Nationaldemokratische Partei } \\
\text { Deutschlands) } \\
\text { (28) AfD (Alternative für } \\
\text { Deutschland) } \\
\text { (29) other party } \\
\text { (-83) invalid vote } \\
\text { (-97) not applicable } \\
\text { (-98) don't know } \\
\text { (-99) no answer }\end{array}$ & $\begin{array}{l}P R \text { vote (alternative-specific): } \\
\text { (1) Intention/recollection of } \\
\text { voting for... } \\
\text { (0) No intention/recollection of } \\
\text { voting for party... } \\
\text { Per vote choice: } \\
\text { (1) CDU/CSU } \\
\text { (2) SPD } \\
\text { (3) The Left } \\
\text { (4) Greens } \\
\text { (5) FDP } \\
\text { remaining observations ignored }\end{array}$ \\
\hline $\begin{array}{l}\text { vn195 (German citizenship } \\
\text { since birth) }\end{array}$ & $\begin{array}{l}\text { „Do you have German } \\
\text { citizenship since you were } \\
\text { born?” } \\
\text { (1) yes } \\
\text { (2) no } \\
(-98) \text { don't know } \\
(-99) \text { no answer }\end{array}$ & $\begin{array}{l}\text { Used to split dataset into an } \\
\text { immigrant voters and } \\
\text { distinguish different immigrant } \\
\text { groups and generation: } \\
\text { Poland } \\
\text { Former USSR } \\
\text { First generation }\end{array}$ \\
\hline $\begin{array}{l}\text { vn198 (Country of birth other } \\
\text { than Germany) }\end{array}$ & $\begin{array}{l}\text { "Please tell me where you were } \\
\text { born." } \\
\text { (1) former German territory } \\
\text { (e.g. Silesia, Eastern Prussia) } \\
\text { (2) Turkey } \\
\text { (3) Italy } \\
\text { (4) Poland }\end{array}$ & \\
\hline
\end{tabular}




\begin{tabular}{|c|c|c|}
\hline & $\begin{array}{l}\text { (5) Former USSR } \\
\text { (6) Greece } \\
\text { (7) Croatia, Serbia, Bosnia and } \\
\text { Herzegovina, former } \\
\text { Yugoslavia } \\
\text { (8) Austria } \\
\text { (9) Slovakia, Czech Republic, } \\
\text { Former Czechoslovakia } \\
\text { (10) Denmark, Sweden, } \\
\text { Norway, Finland } \\
\text { (11) Netherlands } \\
\text { (12) Belgium } \\
\text { (13) France } \\
\text { (14) Switzerland } \\
\text { (15) USA } \\
\text { (16) other country } \\
\text { (-97) not applicable } \\
\text { (-98) don't know } \\
\text { (-99) no answer }\end{array}$ & \\
\hline $\begin{array}{l}\text { vn203 (Father's country of birth } \\
\text { other than Germany) } \\
\text { vn204 (Mother's country of } \\
\text { birth other than Germany) }\end{array}$ & $\begin{array}{l}\text { Example respondent's mother: } \\
\text { "Please tell me where your } \\
\text { mother was born." } \\
\text { (1) former German territory } \\
\text { (e.g. Silesia, Eastern Prussia) } \\
\text { (2) Turkey } \\
\text { (3) Italy } \\
\text { (4) Poland } \\
\text { (5) Former USSR } \\
\text { (6) Greece } \\
\text { (7) Croatia, Serbia, Bosnia and } \\
\text { Herzegovina, former } \\
\text { Yugoslavia } \\
\text { (8) Austria } \\
\text { (9) Slovakia, Czech Republic, } \\
\text { Former Czechoslovakia } \\
\text { (10) Denmark, Sweden, } \\
\text { Norway, Finland } \\
\text { (11) Netherlands } \\
\text { (12) Belgium } \\
\text { (13) France } \\
\text { (14) Switzerland } \\
\text { (15) USA } \\
\text { (16) other country } \\
\text { (-97) not applicable } \\
\text { (-98) don't know } \\
\text { (-99) no answer }\end{array}$ & \\
\hline $\begin{array}{l}\text { v82a-e (Local candidate } \\
\text { knowledge: pre-election survey) } \\
\text { n82a-e (Local candidate } \\
\text { knowledge: post-election } \\
\text { survey) }\end{array}$ & $\begin{array}{l}\text { "Do you know the name of one } \\
\text { or several district candidates } \\
\text { and can you maybe even tell me } \\
\text { for which party these candidates } \\
\text { are running in the Election on }\end{array}$ & $\begin{array}{l}\text { Candidate awareness } \\
\text { (alternative-specific): } \\
\text { (1) Name and party correct } \\
\text { (0) otherwise }\end{array}$ \\
\hline
\end{tabular}




\begin{tabular}{|c|c|c|}
\hline & $\begin{array}{l}\text { September } 222013 \text { ? Please tell } \\
\text { me the name and party of the } \\
\text { candidates" } \\
\text { Per party: } \\
\text { (1) Name and party correct } \\
\text { (2) Name correct, party } \\
\text { incorrect } \\
\text { (3) Name correct, no party } \\
\text { mentioned } \\
\text { (4) Name incorrect, but party } \\
\text { mentioned } \\
\text { (5) Neiter name nor party }\end{array}$ & $\begin{array}{l}\text { Per vote choice: } \\
\text { (1) CDU/CSU } \\
\text { (2) SPD } \\
\text { (3) The Left } \\
\text { (4) Greens } \\
\text { (5) FDP }\end{array}$ \\
\hline $\begin{array}{l}\text { v11aa (Candidate vote: pre- } \\
\text { election survey) } \\
\text { n11aa (Candidate vote: post- } \\
\text { election survey) }\end{array}$ & $\begin{array}{l}\text { Example pre-election study: } \\
\text { “In the Bundestag election you } \\
\text { can cast two votes. The first } \\
\text { vote for a candidate in your } \\
\text { electoral district and a second } \\
\text { vote for a party. Here is a ballot } \\
\text { specimen similarly to the one } \\
\text { you will be provided with forthe } \\
\text { election. How are you going to } \\
\text { vote?” } \\
\text { (21) CDU/CSU (Christlich } \\
\text { Demokratische Union/Christich- } \\
\text { Soziale Union) } \\
\text { (22) SPD (Sozialdemokratische } \\
\text { Partei Deutschlands) } \\
\text { (23) FDP (Freie Demokratische } \\
\text { Partei) } \\
\text { (24) DIE LINKE (DIE LINKE) } \\
\text { (25) GRÜNE (BÜNDNIS } \\
\text { 90/DIE GRÜNEN) } \\
\text { (26) PIRATEN (Piratenpartei } \\
\text { Deutschland) } \\
\text { (27) NPD } \\
\text { (Nationaldemokratische Partei } \\
\text { Deutschlands) } \\
\text { (28) AfD (Alternative für } \\
\text { Deutschland) } \\
\text { (29) other party } \\
\text { (-83) invalid vote } \\
\text { (-97) not applicable } \\
\text { (-98) don't know } \\
\text { (-99) no answer }\end{array}$ & $\begin{array}{l}\text { SMD vote (alternative-specific): } \\
\text { (1) Intention/recollection of } \\
\text { voting for candidate from ... } \\
\text { (0) No intention/recollection of } \\
\text { voting for candidate from... } \\
\text { Per vote choice: } \\
\text { (1) CDU/CSU } \\
\text { (2) SPD } \\
\text { (3) The Left } \\
\text { (4) Greens } \\
\text { (5) FDP }\end{array}$ \\
\hline vn119a (Party Identification) & $\begin{array}{l}\text { "In Germany many people have } \\
\text { a long-term tendency to identify } \\
\text { with a certain political party, } \\
\text { even if they vote sometimes for } \\
\text { a different party. What about } \\
\text { you: Generally speaking, do you } \\
\text { identify with a certain party? } \\
\text { And if so, which party?" }\end{array}$ & $\begin{array}{l}\text { Party identification (alternative- } \\
\text { specific): } \\
\text { (5) very strong identification } \\
\text { with party } \\
\text { (4) strong identification with } \\
\text { party } \\
\text { (3) moderate identification with }\end{array}$ \\
\hline
\end{tabular}




\begin{tabular}{|c|c|c|}
\hline & $\begin{array}{l}\text { (1) CDU/CSU } \\
\text { (2) CDU } \\
\text { (3) CSU } \\
\text { (4) SPD } \\
\text { (5) FDP } \\
\text { (7) DIE LINKE } \\
\text { (6) GRÜNE } \\
\text { (215) PIRATEN (Piratenpartei } \\
\text { Deutschland) } \\
\text { (206) NPD } \\
\text { (322) AfD (Alternative für } \\
\text { Deutschland) } \\
\text { (801) other party } \\
\text { (808) no party } \\
\text { (-98) don't knoe } \\
\text { (-99) no answer }\end{array}$ & \multirow[t]{2}{*}{$\begin{array}{l}\text { party } \\
\text { (2) weak identification with } \\
\text { party } \\
\text { (1) very weak identification } \\
\text { with party } \\
\text { (0) no identification with party } \\
\text { Per vote choice: } \\
\text { (1) CDU/CSU } \\
\text { (2) SPD } \\
\text { (3) The Left } \\
\text { (4) Greens } \\
\text { (5) FDP }\end{array}$} \\
\hline $\begin{array}{l}\text { vn120 (Strength of Party } \\
\text { Identification) }\end{array}$ & $\begin{array}{l}\text { "How strong or weak is your } \\
\text { identification with this party?" } \\
\text { (1) very strongly } \\
\text { (2) rather strongly } \\
\text { (3) moderately } \\
\text { (4) rather weakly } \\
\text { (5) very weakly } \\
\text { (-97) not applicable } \\
\text { (-98) don't know } \\
(-99) \text { no answer }\end{array}$ & \\
\hline vn62 (left-right self-placement) & $\begin{array}{l}\text { "In politics people often talk } \\
\text { about "left" and "right". On a } \\
\text { scale from } 1 \text { till 11, on which } 1 \\
\text { is "left" and } 11 \text { is "right", where } \\
\text { would you place yourself?" } \\
\text { (1) } 1 \text { left } \\
\text { (2) } 2 \\
\text { (3) } 3 \\
\text { (4) } 4 \\
\text { (5) } 5 \\
\text { (6) } 6 \\
\text { (7) } 7 \\
\text { (8) } 8 \\
\text { (9) } 9 \\
\text { (10) } 10 \\
\text { (11) } 11 \text { right } \\
\text { (-97) not applicable } \\
\text { (-98) don't know } \\
\text { (-99) no answer }\end{array}$ & Rile self-placement \\
\hline $\begin{array}{l}\text { v41 (Chancellor preference: pre- } \\
\text { election survey) } \\
\text { n41 (Chancellor preference: } \\
\text { post-election survey) }\end{array}$ & $\begin{array}{l}\text { Example pre-election study: } \\
\text { "Who would you prefer to be } \\
\text { chancellor after the Bundestag } \\
\text { election: Angela Merkel or Peer } \\
\text { Steinbrück?" }\end{array}$ & Chancellor preference \\
\hline
\end{tabular}




\begin{tabular}{|l|l|l|}
\hline & (1) Angela Merkel & \\
& (2) Peer Steinbrück & \\
& (3) neither/nor & \\
& $(-97)$ not applicable & \\
& $(-98)$ don't know & \\
& $(-99)$ no answer & \\
\hline vn7 (Political knowledge: & In the Bundestag election you & System knowledge \\
Candidate and party vote) & will have two votes, a first and a & \\
& second vote. Which of these & (1) correct answer (second vote) \\
& votes determined the seat & (0) incorrect answer \\
& distribution in the Bundestag?" & \\
& (1) the first vote & \\
& (2) the second vote & \\
& (3) both votes are equally & \\
important & \\
& $(-98)$ don't know & \\
& $(-99)$ no answer & \\
\hline
\end{tabular}


Table A2: Main alternative-specific multinomial logit models

\begin{tabular}{|c|c|c|c|c|}
\hline & $\begin{array}{c}\text { Model 1: } \\
\text { Immigrants' } \\
\text { PR votes }\end{array}$ & $\begin{array}{l}\text { Model 2: } \\
\text { Natives' } \\
\text { PR votes }\end{array}$ & $\begin{array}{l}\text { Model 3: } \\
\text { Immigrants' } \\
\text { PR votes }\end{array}$ & $\begin{array}{l}\text { Model 4: } \\
\text { Natives' } \\
\text { PR votes }\end{array}$ \\
\hline \multicolumn{5}{|c|}{ Alternative-specific equation: } \\
\hline $\mathrm{CIO}$ & $\begin{array}{l}1.51 * * \\
(0.49)\end{array}$ & $\begin{array}{l}-0.22 \\
(0.32)\end{array}$ & $\begin{array}{c}0.31 \\
(0.42)\end{array}$ & $\begin{array}{l}-0.42 \\
(0.39)\end{array}$ \\
\hline Candidate awareness & $\begin{array}{l}1.73 * * \\
(0.54)\end{array}$ & $\begin{array}{c}0.86^{* * * *} \\
(0.15)\end{array}$ & $\begin{array}{l}1.44^{*} \\
(0.62)\end{array}$ & $\begin{array}{c}0.84 * * * \\
(0.14)\end{array}$ \\
\hline CIO \# awareness & & & $\begin{array}{c}2.91 * * * \\
(0.87)\end{array}$ & $\begin{array}{c}0.39 \\
(0.62)\end{array}$ \\
\hline Party identification & $\begin{array}{c}0.75 * * * \\
(0.16)\end{array}$ & $\begin{array}{c}0.64 * * * \\
(0.05)\end{array}$ & $\begin{array}{c}0.77 * * * \\
(0.17)\end{array}$ & $\begin{array}{c}0.64 * * * \\
(0.05)\end{array}$ \\
\hline SMD vote & $\begin{array}{l}0.64+ \\
(0.34)\end{array}$ & $\begin{array}{l}1.39 * * * \\
(0.12)\end{array}$ & $\begin{array}{l}0.62+ \\
(0.38)\end{array}$ & $\begin{array}{c}1.39 * * * \\
(0.12)\end{array}$ \\
\hline CIO list share & $\begin{array}{c}0.97 \\
(1.59) \\
\end{array}$ & $\begin{array}{l}-0.35 \\
(0.50)\end{array}$ & $\begin{array}{c}0.97 \\
(1.70) \\
\end{array}$ & $\begin{array}{l}-0.38 \\
(0.52) \\
\end{array}$ \\
\hline \multicolumn{5}{|c|}{ Individual-specific: SPD vote } \\
\hline Post-election survey & $\begin{array}{c}0.18 \\
(0.74)\end{array}$ & $\begin{array}{c}0.11 \\
(0.25)\end{array}$ & $\begin{array}{c}0.15 \\
(0.73)\end{array}$ & $\begin{array}{c}0.10 \\
(0.25)\end{array}$ \\
\hline System knowledge & $\begin{array}{c}0.37 \\
(0.71)\end{array}$ & $\begin{array}{l}-0.52^{*} \\
(0.26)\end{array}$ & $\begin{array}{c}0.42 \\
(0.71)\end{array}$ & $\begin{array}{l}-0.51^{*} \\
(0.26)\end{array}$ \\
\hline Former USSR $^{a}$ & $\begin{array}{l}-0.62 \\
(0.92)\end{array}$ & (dropped) & $\begin{array}{l}-0.65 \\
(0.87)\end{array}$ & (dropped) \\
\hline Poland $^{\mathrm{a}}$ & $\begin{array}{c}0.77 \\
(0.86)\end{array}$ & (dropped) & $\begin{array}{c}0.66 \\
(0.88)\end{array}$ & (dropped) \\
\hline First generation & $\begin{array}{c}0.41 \\
(0.85)\end{array}$ & (dropped) & $\begin{array}{c}0.46 \\
(0.87)\end{array}$ & (dropped) \\
\hline Steinbrück $^{\mathrm{b}}$ & $\begin{array}{c}17.17 * * * \\
(0.85)\end{array}$ & $\begin{array}{c}2.98 * * * \\
(0.44)\end{array}$ & $\begin{array}{c}16.82 * * * \\
(0.79)\end{array}$ & $\begin{array}{c}2.99 * * * \\
(0.44)\end{array}$ \\
\hline $\begin{array}{l}\text { Neither Merkel nor } \\
\text { Steinbrück }\end{array}$ & $\begin{array}{l}3.17 * * * \\
(0.86)\end{array}$ & $\begin{array}{c}2.03 * * * \\
(0.49)\end{array}$ & $\begin{array}{c}3.25^{* * * *} \\
(0.87)\end{array}$ & $\begin{array}{c}2.03 * * * \\
(0.49)\end{array}$ \\
\hline Rile self-placement & $\begin{array}{l}-0.43^{*} \\
(0.21)\end{array}$ & $\begin{array}{c}-0.23^{* *} \\
(0.08)\end{array}$ & $\begin{array}{l}-0.41^{*} \\
(0.20)\end{array}$ & $\begin{array}{c}-0.23 * * \\
(0.08)\end{array}$ \\
\hline INTERCEPT & $\begin{array}{c}0.30 \\
(1.47) \\
\end{array}$ & $\begin{array}{c}0.68 \\
(0.50) \\
\end{array}$ & $\begin{array}{c}0.26 \\
(1.54) \\
\end{array}$ & $\begin{array}{c}0.68 \\
(0.50) \\
\end{array}$ \\
\hline
\end{tabular}

\section{Individual-specific: The Left}

vote

Post-election survey

System knowledge

Former USSR $^{\mathrm{a}}$

Poland $^{\text {a }}$

First generation

0.33
$(0.85)$
1.08
$(0.81)$
$-1.70+$
$(1.01)$
1.45
$(0.88)$

0.22 $(0.81)$

$$
\begin{gathered}
-0.05 \\
(0.27) \\
-0.73 * * \\
(0.27)
\end{gathered}
$$

(dropped)

(dropped)

(dropped)
$-0.09$

(0.93)

1.17

$(0.83)$

$-1.59+$

$(0.93)$

1.29

(0.88)

0.22

(0.81)
$-0.04$

(0.27)

$-0.72 * *$

(0.27)

(dropped)

(dropped)

(dropped) 


\begin{tabular}{|c|c|c|c|c|}
\hline Steinbrück ${ }^{b}$ & $\begin{array}{l}16.21 * * * \\
(0.94)\end{array}$ & $\begin{array}{l}2.75 * * * \\
(0.48)\end{array}$ & $\begin{array}{l}15.79 * * * \\
(0.88)\end{array}$ & $\begin{array}{c}2.75 * * * \\
(0.48)\end{array}$ \\
\hline Neither Merkel nor & $4.63 * * *$ & $2.69 * * *$ & $4.77 * * *$ & $2.69 * * *$ \\
\hline Steinbrück ${ }^{\mathrm{b}}$ & $(0.95)$ & $(0.44)$ & $(0.96)$ & $(0.45)$ \\
\hline Rile self-placement & $\begin{array}{l}-0.84^{*} \\
(0.33)\end{array}$ & $\begin{array}{c}-0.78 * * * \\
(0.09)\end{array}$ & $\begin{array}{l}-0.83^{*} \\
(0.38)\end{array}$ & $\begin{array}{c}-0.78^{* * * *} \\
(0.09)\end{array}$ \\
\hline INTERCEPT & $\begin{array}{l}1.36 \\
(1.87)\end{array}$ & $\begin{array}{c}3.25^{* * *} \\
(0.54)\end{array}$ & $\begin{array}{c}1.67 \\
(2.21)\end{array}$ & $\begin{array}{c}3.25 * * * \\
(0.54)\end{array}$ \\
\hline \multicolumn{5}{|c|}{$\begin{array}{l}\text { Individual-specific: Greens } \\
\text { vote }\end{array}$} \\
\hline Post-election survey & $\begin{array}{c}0.13 \\
(0.77)\end{array}$ & $\begin{array}{c}0.06 \\
(0.31)\end{array}$ & $\begin{array}{c}0.00 \\
(0.80)\end{array}$ & $\begin{array}{c}0.06 \\
(0.31)\end{array}$ \\
\hline System knowledge & $\begin{array}{c}1.29 \\
(0.80)\end{array}$ & $\begin{array}{l}-0.20 \\
(0.28)\end{array}$ & $\begin{array}{l}1.40+ \\
(0.84)\end{array}$ & $\begin{array}{l}-0.19 \\
(0.28)\end{array}$ \\
\hline Former USSR ${ }^{a}$ & $\begin{array}{l}-1.41 \\
(0.95)\end{array}$ & (dropped) & $\begin{array}{l}-1.46 \\
(1.05)\end{array}$ & (dropped) \\
\hline Poland $^{\mathrm{a}}$ & $\begin{array}{c}0.56 \\
(0.93)\end{array}$ & (dropped) & $\begin{array}{c}0.77 \\
(0.93)\end{array}$ & (dropped) \\
\hline First generation & $\begin{array}{l}-0.86 \\
(0.77)\end{array}$ & (dropped) & $\begin{array}{l}-1.00 \\
(0.82)\end{array}$ & (dropped) \\
\hline Steinbrück ${ }^{\mathrm{b}}$ & $\begin{array}{l}16.92 * * * \\
(0.81)\end{array}$ & $\begin{array}{l}2.81 * * * \\
(0.42)\end{array}$ & $\begin{array}{l}16.32 * * * \\
(0.74)\end{array}$ & $\begin{array}{l}2.81 * * * \\
(0.42)\end{array}$ \\
\hline $\begin{array}{l}\text { Neither Merkel nor } \\
\text { Steinbrück }{ }^{\mathrm{b}}\end{array}$ & $\begin{array}{c}2.83 * * \\
(0.99)\end{array}$ & $\begin{array}{c}1.85^{* * * *} \\
(0.48)\end{array}$ & $\begin{array}{c}3.43^{* * *} \\
(0.97)\end{array}$ & $\begin{array}{c}1.84 * * * \\
(0.48)\end{array}$ \\
\hline Rile self-placement & $\begin{array}{l}-0.34+ \\
(0.19)\end{array}$ & $\begin{array}{c}-0.33 * * * \\
(0.09)\end{array}$ & $\begin{array}{l}-0.47^{*} \\
(0.21)\end{array}$ & $\begin{array}{c}-0.33^{* * * *} \\
(0.09)\end{array}$ \\
\hline INTERCEPT & $\begin{array}{c}0.40 \\
(1.32)\end{array}$ & $\begin{array}{l}1.51^{*} \\
(0.60)\end{array}$ & $\begin{array}{c}0.78 \\
(1.41)\end{array}$ & $\begin{array}{l}1.49^{*} \\
(0.60)\end{array}$ \\
\hline \multicolumn{5}{|c|}{ Individual-specific: FDP vote } \\
\hline Post-election survey & $\begin{array}{l}-0.34 \\
(1.34)\end{array}$ & $\begin{array}{l}-0.41 \\
(0.36)\end{array}$ & $\begin{array}{l}-0.41 \\
(1.29)\end{array}$ & $\begin{array}{l}-0.41 \\
(0.36)\end{array}$ \\
\hline System knowledge & $\begin{array}{l}-1.14 \\
(0.84)\end{array}$ & $\begin{array}{c}-0.92 * * \\
(0.31)\end{array}$ & $\begin{array}{l}-1.15 \\
(0.82)\end{array}$ & $\begin{array}{c}-0.93 * * \\
(0.31)\end{array}$ \\
\hline Former USSR $^{\mathrm{a}}$ & $\begin{array}{c}-16.18^{* * * *} \\
(0.70)\end{array}$ & (dropped) & $\begin{array}{c}-15.87 * * * \\
(0.67)\end{array}$ & (dropped) \\
\hline Poland $^{\mathrm{a}}$ & $\begin{array}{l}1.78+ \\
(1.07)\end{array}$ & (dropped) & $\begin{array}{l}1.83+ \\
(1.06)\end{array}$ & (dropped) \\
\hline First generation & $\begin{array}{l}-0.69 \\
(0.88)\end{array}$ & (dropped) & $\begin{array}{l}-0.89 \\
(0.89)\end{array}$ & (dropped) \\
\hline Steinbrück ${ }^{b}$ & $\begin{array}{l}16.95 * * * \\
(1.49)\end{array}$ & $\begin{array}{l}1.76^{* *} \\
(0.64)\end{array}$ & $\begin{array}{c}16.61 * * * \\
(1.38)\end{array}$ & $\begin{array}{l}1.76^{* *} \\
(0.64)\end{array}$ \\
\hline $\begin{array}{l}\text { Neither Merkel nor } \\
\text { Steinbrück }{ }^{\mathrm{b}}\end{array}$ & $\begin{array}{l}2.58^{*} \\
(1.21)\end{array}$ & $\begin{array}{l}-0.64 \\
(0.92)\end{array}$ & $\begin{array}{l}2.70^{*} \\
(1.19)\end{array}$ & $\begin{array}{l}-0.63 \\
(0.92)\end{array}$ \\
\hline Rile self-placement & $\begin{array}{c}0.35 \\
(0.28)\end{array}$ & $\begin{array}{c}0.08 \\
(0.10)\end{array}$ & $\begin{array}{c}0.37 \\
(0.27)\end{array}$ & $\begin{array}{c}0.09 \\
(0.10)\end{array}$ \\
\hline INTERCEPT & $\begin{array}{l}-2.84 \\
(2.04)\end{array}$ & $\begin{array}{l}-0.32 \\
(0.71)\end{array}$ & $\begin{array}{l}-2.87 \\
(2.04)\end{array}$ & $\begin{array}{l}-0.33 \\
(0.72)\end{array}$ \\
\hline $\mathrm{N}$ (alternatives) & 1235 & 10930 & 1235 & 10930 \\
\hline $\mathrm{N}($ cases $)$ & 247 & 2154 & 247 & 2154 \\
\hline
\end{tabular}


Note: $+\mathrm{p}<0.10,{ }^{*} \mathrm{p}<0.05, * * \mathrm{p}<0.01, * * * \mathrm{p}<0.001$; robust standard errors, clustered on constituencies, are shown in parentheses; models estimated on weighted data to compensate for an oversampling of East German regions and to make the data more representative of the socio-structural outlook of the German voting age population (weight variable in the GLES 2013 dataset: w_ipfges_2); ${ }^{a}$ reference category is remaining immigrant groups; ${ }^{b}$ reference group is Merkel 
Table A3: Robustness alternative-specific multinomial logit models (unweighted data)

\begin{tabular}{|c|c|c|c|c|}
\hline & $\begin{array}{c}\text { Model 1: } \\
\text { Immigrants' } \\
\text { PR votes }\end{array}$ & $\begin{array}{l}\text { Model 2: } \\
\text { Natives' } \\
\text { PR votes }\end{array}$ & $\begin{array}{c}\text { Model 3: } \\
\text { Immigrants' } \\
\text { PR votes }\end{array}$ & $\begin{array}{l}\text { Model 4: } \\
\text { Natives' } \\
\text { PR votes }\end{array}$ \\
\hline \multicolumn{5}{|l|}{$\begin{array}{l}\text { Alternative-specific } \\
\text { equation: }\end{array}$} \\
\hline $\mathrm{CIO}$ & $\begin{array}{l}1.29 * \\
(0.50)\end{array}$ & $\begin{array}{l}-0.07 \\
(0.26)\end{array}$ & $\begin{array}{c}0.17 \\
(0.40)\end{array}$ & $\begin{array}{l}-0.41 \\
(0.33)\end{array}$ \\
\hline Candidate awareness & $\begin{array}{l}1.56 * * \\
(0.52)\end{array}$ & $\begin{array}{c}0.91^{* * *} \\
(0.13)\end{array}$ & $\begin{array}{l}1.29^{*} \\
(0.57)\end{array}$ & $\begin{array}{c}0.88 * * * \\
(0.13)\end{array}$ \\
\hline CIO \# awareness & & & $\begin{array}{l}2.53 * * \\
(0.93)\end{array}$ & $\begin{array}{c}0.66 \\
(0.52)\end{array}$ \\
\hline Party identification & $\begin{array}{c}0.71 * * * \\
(0.15)\end{array}$ & $\begin{array}{c}0.63 * * * \\
(0.05)\end{array}$ & $\begin{array}{c}0.71 * * * \\
(0.16)\end{array}$ & $\begin{array}{c}0.63 * * * \\
(0.05)\end{array}$ \\
\hline SMD vote & $\begin{array}{l}0.96^{* *} \\
(0.31)\end{array}$ & $\begin{array}{c}1.54 * * * \\
(0.11)\end{array}$ & $\begin{array}{l}0.96^{* *} \\
(0.33)\end{array}$ & $\begin{array}{c}1.54 * * * \\
(0.11)\end{array}$ \\
\hline CIO list share & $\begin{array}{c}1.28 \\
(1.29)\end{array}$ & $\begin{array}{l}-0.05 \\
(0.47)\end{array}$ & $\begin{array}{c}1.29 \\
(1.44)\end{array}$ & $\begin{array}{l}-0.13 \\
(0.49)\end{array}$ \\
\hline \multicolumn{5}{|l|}{$\begin{array}{l}\text { Individual-specific: } \\
\text { SPD vote }\end{array}$} \\
\hline Post-election survey & $\begin{array}{c}0.41 \\
(0.67)\end{array}$ & $\begin{array}{c}0.19 \\
(0.24)\end{array}$ & $\begin{array}{c}0.39 \\
(0.64)\end{array}$ & $\begin{array}{c}0.18 \\
(0.24)\end{array}$ \\
\hline System knowledge & $\begin{array}{c}0.48 \\
(0.61)\end{array}$ & $\begin{array}{c}-0.60 * * \\
(0.22)\end{array}$ & $\begin{array}{c}0.48 \\
(0.61)\end{array}$ & $\begin{array}{c}-0.60 * * \\
(0.22)\end{array}$ \\
\hline Former USSR $^{\mathrm{a}}$ & $\begin{array}{l}-0.93 \\
(0.85)\end{array}$ & (dropped) & $\begin{array}{l}-0.95 \\
(0.81)\end{array}$ & (dropped) \\
\hline Poland $^{\text {a }}$ & $\begin{array}{c}0.81 \\
(0.75)\end{array}$ & (dropped) & $\begin{array}{c}0.80 \\
(0.77)\end{array}$ & (dropped) \\
\hline First generation & $\begin{array}{c}0.14 \\
(0.78)\end{array}$ & (dropped) & $\begin{array}{c}0.15 \\
(0.80)\end{array}$ & (dropped) \\
\hline Steinbrück ${ }^{b}$ & $\begin{array}{l}16.04 * * * \\
(0.72)\end{array}$ & $\begin{array}{l}2.88 * * * \\
(0.42)\end{array}$ & $\begin{array}{l}15.98 * * * \\
(0.64)\end{array}$ & $\begin{array}{l}2.89 * * * \\
(0.42)\end{array}$ \\
\hline $\begin{array}{l}\text { Neither Merkel nor } \\
\text { Steinbrück }{ }^{\mathrm{b}}\end{array}$ & $\begin{array}{c}2.79 * * * \\
(0.84)\end{array}$ & $\begin{array}{c}1.84 * * * \\
(0.38)\end{array}$ & $\begin{array}{l}2.81 * * \\
(0.87)\end{array}$ & $\begin{array}{c}1.84 * * * \\
(0.38)\end{array}$ \\
\hline Rile self-placement & $\begin{array}{r}-0.49^{*} \\
(0.19)\end{array}$ & $\begin{array}{c}-0.19 * * \\
(0.07)\end{array}$ & $\begin{array}{l}-0.47^{*} \\
(0.19)\end{array}$ & $\begin{array}{c}-0.19^{* *} \\
(0.07)\end{array}$ \\
\hline INTERCEPT & $\begin{array}{c}0.63 \\
(1.24) \\
\end{array}$ & $\begin{array}{c}0.42 \\
(0.47) \\
\end{array}$ & $\begin{array}{c}0.64 \\
(1.30) \\
\end{array}$ & $\begin{array}{c}0.41 \\
(0.47) \\
\end{array}$ \\
\hline \multicolumn{5}{|c|}{$\begin{array}{l}\text { Individual-specific: The } \\
\text { Left vote }\end{array}$} \\
\hline Post-election survey & $\begin{array}{c}0.57 \\
(0.72)\end{array}$ & $\begin{array}{c}0.11 \\
(0.27)\end{array}$ & $\begin{array}{c}0.32 \\
(0.78)\end{array}$ & $\begin{array}{c}0.10 \\
(0.27)\end{array}$ \\
\hline System knowledge & $\begin{array}{l}1.58^{*} \\
(0.74)\end{array}$ & $\begin{array}{c}-1.09 * * * \\
(0.23)\end{array}$ & $\begin{array}{l}1.70^{*} \\
(0.78)\end{array}$ & $\begin{array}{c}-1.07 * * * \\
(0.23)\end{array}$ \\
\hline Former USSR $^{a}$ & $\begin{array}{l}-1.38 \\
(0.85)\end{array}$ & (dropped) & $\begin{array}{l}-1.33 \\
(0.82)\end{array}$ & (dropped) \\
\hline Poland $^{\mathrm{a}}$ & $\begin{array}{c}0.76 \\
(0.88)\end{array}$ & (dropped) & $\begin{array}{c}0.68 \\
(0.91)\end{array}$ & (dropped) \\
\hline
\end{tabular}




\begin{tabular}{|c|c|c|c|c|}
\hline First generation & $\begin{array}{l}-0.65 \\
(0.72)\end{array}$ & (dropped) & $\begin{array}{l}-0.69 \\
(0.73)\end{array}$ & (dropped) \\
\hline Steinbrück ${ }^{b}$ & $\begin{array}{c}14.78 * * * \\
(0.91)\end{array}$ & $\begin{array}{l}2.77 * * * \\
(0.46)\end{array}$ & $\begin{array}{c}14.64 * * * \\
(0.87)\end{array}$ & $\begin{array}{c}2.77 * * * \\
(0.46)\end{array}$ \\
\hline $\begin{array}{l}\text { Neither Merkel nor } \\
\text { Steinbrück }{ }^{b}\end{array}$ & $\begin{array}{c}3.89 * * * \\
(0.96)\end{array}$ & $\begin{array}{c}2.56^{* * * *} \\
(0.40)\end{array}$ & $\begin{array}{c}4.15 * * * \\
(0.99)\end{array}$ & $\begin{array}{c}2.57 * * * \\
(0.40)\end{array}$ \\
\hline Rile self-placement & $\begin{array}{c}-0.78 * * \\
(0.29)\end{array}$ & $\begin{array}{c}-0.66^{* * *} \\
(0.09)\end{array}$ & $\begin{array}{l}-0.80^{*} \\
(0.32)\end{array}$ & $\begin{array}{c}-0.66 * * * \\
(0.09)\end{array}$ \\
\hline INTERCEPT & $\begin{array}{c}1.98 \\
(1.60)\end{array}$ & $\begin{array}{c}2.71 * * * \\
(0.57) \\
\end{array}$ & $\begin{array}{c}2.24 \\
(1.77) \\
\end{array}$ & $\begin{array}{c}2.71 * * * \\
(0.57)\end{array}$ \\
\hline \multicolumn{5}{|l|}{$\begin{array}{l}\text { Individual-specific: } \\
\text { Greens vote }\end{array}$} \\
\hline Post-election survey & $\begin{array}{c}0.21 \\
(0.66)\end{array}$ & $\begin{array}{c}0.05 \\
(0.28)\end{array}$ & $\begin{array}{c}0.14 \\
(0.66)\end{array}$ & $\begin{array}{c}0.04 \\
(0.28)\end{array}$ \\
\hline System knowledge & $\begin{array}{l}1.12+ \\
(0.67)\end{array}$ & $\begin{array}{l}-0.33 \\
(0.25)\end{array}$ & $\begin{array}{l}1.24+ \\
(0.71)\end{array}$ & $\begin{array}{l}-0.33 \\
(0.25)\end{array}$ \\
\hline Former USSR ${ }^{a}$ & $\begin{array}{l}-1.53+ \\
(0.86)\end{array}$ & (dropped) & $\begin{array}{l}-1.60+ \\
(0.94)\end{array}$ & (dropped) \\
\hline Poland $^{\mathrm{a}}$ & $\begin{array}{c}0.57 \\
(0.83)\end{array}$ & (dropped) & $\begin{array}{c}0.73 \\
(0.84)\end{array}$ & (dropped) \\
\hline First generation & $\begin{array}{l}-1.08 \\
(0.71)\end{array}$ & (dropped) & $\begin{array}{l}-1.06 \\
(0.74)\end{array}$ & (dropped) \\
\hline Steinbrück ${ }^{\mathrm{b}}$ & $\begin{array}{c}15.90 * * * \\
(0.64)\end{array}$ & $\begin{array}{c}2.68 * * * \\
(0.42)\end{array}$ & $\begin{array}{c}15.67 * * * \\
(0.57)\end{array}$ & $\begin{array}{c}2.69 * * * \\
(0.42)\end{array}$ \\
\hline $\begin{array}{l}\text { Neither Merkel nor } \\
\text { Steinbrück } \mathrm{b}\end{array}$ & $\begin{array}{c}2.75 * * \\
(0.94)\end{array}$ & $\begin{array}{c}1.62 * * * \\
(0.40)\end{array}$ & $\begin{array}{c}3.23 * * * \\
(0.93)\end{array}$ & $\begin{array}{c}1.61 * * * \\
(0.40)\end{array}$ \\
\hline Rile self-placement & $\begin{array}{l}-0.32+ \\
(0.17)\end{array}$ & $\begin{array}{c}-0.24^{* *} \\
(0.09)\end{array}$ & $\begin{array}{l}-0.43^{*} \\
(0.18)\end{array}$ & $\begin{array}{c}-0.23^{* *} \\
(0.09)\end{array}$ \\
\hline INTERCEPT & $\begin{array}{c}0.67 \\
(1.11) \\
\end{array}$ & $\begin{array}{c}0.86 \\
(0.58) \\
\end{array}$ & $\begin{array}{c}0.94 \\
(1.17) \\
\end{array}$ & $\begin{array}{c}0.84 \\
(0.59) \\
\end{array}$ \\
\hline \multicolumn{5}{|l|}{$\begin{array}{l}\text { Individual-specific: } \\
\text { FDP vote }\end{array}$} \\
\hline Post-election survey & $\begin{array}{l}-0.25 \\
(1.15)\end{array}$ & $\begin{array}{l}-0.49 \\
(0.31)\end{array}$ & $\begin{array}{l}-0.32 \\
(1.12)\end{array}$ & $\begin{array}{l}-0.49 \\
(0.31)\end{array}$ \\
\hline System knowledge & $\begin{array}{l}-1.02 \\
(0.78)\end{array}$ & $\begin{array}{l}-0.71^{*} \\
(0.30)\end{array}$ & $\begin{array}{l}-1.01 \\
(0.77)\end{array}$ & $\begin{array}{l}-0.71^{*} \\
(0.30)\end{array}$ \\
\hline Former USSR ${ }^{a}$ & $\begin{array}{c}-15.12 * * * \\
(0.64)\end{array}$ & (dropped) & $\begin{array}{c}-15.07 * * * \\
(0.63)\end{array}$ & (dropped) \\
\hline Poland $^{\mathrm{a}}$ & $\begin{array}{l}1.81 \\
(1.11)\end{array}$ & (dropped) & $\begin{array}{l}1.86+ \\
(1.12)\end{array}$ & (dropped) \\
\hline First generation & $\begin{array}{l}-0.97 \\
(0.85)\end{array}$ & (dropped) & $\begin{array}{l}-1.09 \\
(0.85)\end{array}$ & (dropped) \\
\hline Steinbrück ${ }^{\mathrm{b}}$ & $\begin{array}{l}15.73 * * * \\
(1.16)\end{array}$ & $\begin{array}{l}1.64 * * \\
(0.61)\end{array}$ & $\begin{array}{l}15.65^{* * * *} \\
(1.08)\end{array}$ & $\begin{array}{l}1.64 * * \\
(0.61)\end{array}$ \\
\hline $\begin{array}{l}\text { Neither Merkel nor } \\
\text { Steinbrück }{ }^{b}\end{array}$ & $\begin{array}{l}2.41^{*} \\
(1.23)\end{array}$ & $\begin{array}{l}-1.09 \\
(0.84)\end{array}$ & $\begin{array}{l}2.54 * \\
(1.22)\end{array}$ & $\begin{array}{l}-1.08 \\
(0.84)\end{array}$ \\
\hline Rile self-placement & $\begin{array}{c}0.33 \\
(0.22)\end{array}$ & $\begin{array}{l}0.20^{*} \\
(0.09)\end{array}$ & $\begin{array}{c}0.33 \\
(0.22)\end{array}$ & $\begin{array}{l}0.20^{*} \\
(0.09)\end{array}$ \\
\hline INTERCEPT & -2.57 & $-1.19+$ & -2.54 & $-1.21+$ \\
\hline
\end{tabular}




\begin{tabular}{lcccc} 
& $(1.68)$ & $(0.69)$ & $(1.73)$ & $(0.69)$ \\
\hline $\mathrm{N}$ (alternatives) & 1235 & 10930 & 1235 & 10930 \\
$\mathrm{~N}$ (cases) & 247 & 2154 & 247 & 2154 \\
Bic & 571.73 & 2474.21 & 569.54 & 2480.92 \\
\hline
\end{tabular}

Note: $+\mathrm{p}<0.10,{ }^{*} \mathrm{p}<0.05,{ }^{* *} \mathrm{p}<0.01,{ }^{* * *} \mathrm{p}<0.001$; robust standard errors, clustered on constituencies, are shown in parentheses; models estimated on unweighted data; ${ }^{a}$ reference category is remaining immigrant groups; ${ }^{b}$ reference group is Merkel 
Table A4: Robustness alternative-specific multinomial logit models (visible CIO)

\begin{tabular}{lcccc}
\hline & $\begin{array}{c}\text { Model 1: } \\
\text { Immigrants, } \\
\text { PR votes }\end{array}$ & $\begin{array}{c}\text { Model 2: } \\
\text { Natives' } \\
\text { PR votes }\end{array}$ & $\begin{array}{c}\text { Model 3: } \\
\text { Immigrants' } \\
\text { PR votes }\end{array}$ & $\begin{array}{c}\text { Model 4: } \\
\text { Natives' } \\
\text { PR votes }\end{array}$ \\
\hline $\begin{array}{l}\text { Alternative-specific } \\
\text { equation: }\end{array}$ & & & & \\
Visible CIO & $1.64^{* *}$ & -0.15 & 0.32 & -0.20 \\
& $(0.55)$ & $(0.30)$ & $(0.54)$ & $(0.33)$ \\
Candidate awareness & $1.81^{* * *}$ & $0.86^{* * *}$ & $1.54^{* *}$ & $0.86^{* * *}$ \\
& $(0.55)$ & $(0.15)$ & $(0.58)$ & $(0.14)$ \\
Visible CIO \# awareness & & & $2.98^{* *}$ & 0.11 \\
& & & $(0.95)$ & $(0.64)$ \\
Party identification & $0.74^{* * *}$ & $0.64^{* * *}$ & $0.75^{* * *}$ & $0.64 * * *$ \\
& $(0.16)$ & $(0.05)$ & $(0.17)$ & $(0.05)$ \\
SMD vote & $0.66^{*}$ & $1.39^{* * *}$ & $0.69+$ & $1.39 * * *$ \\
& $(0.33)$ & $(0.12)$ & $(0.37)$ & $(0.12)$ \\
CIO list share & 1.20 & -0.38 & 1.22 & -0.39 \\
& $(1.75)$ & $(0.51)$ & $(1.79)$ & $(0.53)$ \\
\hline
\end{tabular}

Individual-specific:

SPD vote

\begin{tabular}{|c|c|c|c|c|}
\hline Post-election survey & $\begin{array}{c}0.16 \\
(0.73)\end{array}$ & $\begin{array}{c}0.12 \\
(0.25)\end{array}$ & $\begin{array}{c}0.18 \\
(0.72)\end{array}$ & $\begin{array}{c}0.11 \\
(0.25)\end{array}$ \\
\hline System knowledge & 0.41 & $-0.52 *$ & 0.43 & $-0.52 *$ \\
\hline & $(0.72)$ & $(0.26)$ & $(0.72)$ & $(0.26)$ \\
\hline Former USSR $^{\mathrm{a}}$ & $\begin{array}{l}-0.62 \\
(0.91)\end{array}$ & (dropped) & $\begin{array}{l}-0.67 \\
(0.88)\end{array}$ & (dropped) \\
\hline Poland $^{\mathrm{a}}$ & $\begin{array}{c}0.87 \\
(0.87)\end{array}$ & (dropped) & $\begin{array}{c}0.69 \\
(0.89)\end{array}$ & (dropped) \\
\hline First generation & $\begin{array}{c}0.44 \\
(0.86)\end{array}$ & (dropped) & $\begin{array}{c}0.42 \\
(0.87)\end{array}$ & (dropped) \\
\hline Steinbrück ${ }^{b}$ & $\begin{array}{c}16.89 * * * \\
(0.75)\end{array}$ & $\begin{array}{c}2.98 * * * \\
(0.44)\end{array}$ & $\begin{array}{c}16.70 * * * \\
(0.78)\end{array}$ & $\begin{array}{c}2.98 * * * \\
(0.44)\end{array}$ \\
\hline $\begin{array}{l}\text { Neither Merkel nor } \\
\text { Steinbrück }{ }^{b}\end{array}$ & $\begin{array}{c}3.10 * * * \\
(0.86)\end{array}$ & $\begin{array}{c}2.03 * * * \\
(0.49)\end{array}$ & $\begin{array}{c}3.21 * * * \\
(0.88)\end{array}$ & $\begin{array}{c}2.02 * * * \\
(0.49)\end{array}$ \\
\hline Rile self-placement & $\begin{array}{l}-0.44^{*} \\
(0.22)\end{array}$ & $\begin{array}{c}-0.23^{* *} \\
(0.08)\end{array}$ & $\begin{array}{l}-0.41+ \\
(0.21)\end{array}$ & $\begin{array}{c}-0.23 * * \\
(0.08)\end{array}$ \\
\hline INTERCEPT & $\begin{array}{c}0.30 \\
(1.50) \\
\end{array}$ & $\begin{array}{c}0.67 \\
(0.50) \\
\end{array}$ & $\begin{array}{c}0.27 \\
(1.56) \\
\end{array}$ & $\begin{array}{c}0.67 \\
(0.50)\end{array}$ \\
\hline \multicolumn{5}{|c|}{$\begin{array}{l}\text { Individual-specific: The } \\
\text { Left vote }\end{array}$} \\
\hline Post-election survey & $\begin{array}{c}0.29 \\
(0.83)\end{array}$ & $\begin{array}{l}-0.04 \\
(0.27)\end{array}$ & $\begin{array}{c}0.00 \\
(0.89)\end{array}$ & $\begin{array}{l}-0.04 \\
(0.27)\end{array}$ \\
\hline System knowledge & $\begin{array}{l}1.15 \\
(0.82)\end{array}$ & $\begin{array}{c}-0.73^{* *} \\
(0.27)\end{array}$ & $\begin{array}{l}1.20 \\
(0.81)\end{array}$ & $\begin{array}{c}-0.73 * * \\
(0.27)\end{array}$ \\
\hline Former USSR $^{\mathrm{a}}$ & $\begin{array}{l}-1.63+ \\
(0.98)\end{array}$ & (dropped) & $\begin{array}{l}-1.74+ \\
(0.93)\end{array}$ & (dropped) \\
\hline Poland $^{\mathrm{a}}$ & $\begin{array}{c}1.38 \\
(0.90)\end{array}$ & (dropped) & $\begin{array}{l}1.11 \\
(0.86)\end{array}$ & (dropped) \\
\hline
\end{tabular}




\begin{tabular}{|c|c|c|c|c|}
\hline First generation & $\begin{array}{c}0.10 \\
(0.79)\end{array}$ & (dropped) & $\begin{array}{c}0.11 \\
(0.76)\end{array}$ & (dropped) \\
\hline Steinbrück ${ }^{\mathrm{b}}$ & $\begin{array}{c}15.87 * * * \\
(0.81)\end{array}$ & $\begin{array}{c}2.75^{* * * *} \\
(0.47)\end{array}$ & $\begin{array}{c}15.50 * * * \\
(0.79)\end{array}$ & $\begin{array}{c}2.75 * * * \\
(0.48)\end{array}$ \\
\hline $\begin{array}{l}\text { Neither Merkel nor } \\
\text { Steinbrück }{ }^{b}\end{array}$ & $\begin{array}{c}4.66^{* * *} \\
(0.93)\end{array}$ & $\begin{array}{c}2.69 * * * \\
(0.44)\end{array}$ & $\begin{array}{c}4.64 * * * \\
(0.94)\end{array}$ & $\begin{array}{c}2.69 * * * \\
(044)\end{array}$ \\
\hline Rile self-placement & $\begin{array}{l}-0.81^{*} \\
(0.37)\end{array}$ & $\begin{array}{c}-0.78^{* * *} \\
(0.09)\end{array}$ & $\begin{array}{l}-0.85^{*} \\
(0.37)\end{array}$ & $\begin{array}{c}-0.78^{* * *} \\
(0.09)\end{array}$ \\
\hline INTERCEPT & $\begin{array}{c}1.36 \\
(1.98)\end{array}$ & $\begin{array}{c}3.24 * * * \\
(0.54)\end{array}$ & $\begin{array}{l}1.93 \\
(2.08)\end{array}$ & $\begin{array}{c}3.24 * * * \\
(0.54)\end{array}$ \\
\hline \multicolumn{5}{|l|}{$\begin{array}{l}\text { Individual-specific: } \\
\text { Greens vote }\end{array}$} \\
\hline Post-election survey & $\begin{array}{c}0.15 \\
(0.76)\end{array}$ & $\begin{array}{c}0.06 \\
(0.31)\end{array}$ & $\begin{array}{c}0.08 \\
(0.80)\end{array}$ & $\begin{array}{c}0.06 \\
(0.31)\end{array}$ \\
\hline System knowledge & $\begin{array}{l}1.37+ \\
(0.82)\end{array}$ & $\begin{array}{l}-0.18 \\
(0.29)\end{array}$ & $\begin{array}{l}1.36 \\
(0.87)\end{array}$ & $\begin{array}{l}-0.18 \\
(0.29)\end{array}$ \\
\hline Former USSR $^{a}$ & $\begin{array}{l}-1.40 \\
(0.97)\end{array}$ & (dropped) & $\begin{array}{l}-1.47 \\
(1.11)\end{array}$ & (dropped) \\
\hline Poland $^{\mathrm{a}}$ & $\begin{array}{c}0.60 \\
(0.93)\end{array}$ & (dropped) & $\begin{array}{c}0.78 \\
(0.95)\end{array}$ & (dropped) \\
\hline First generation & $\begin{array}{l}-0.89 \\
(0.77)\end{array}$ & (dropped) & $\begin{array}{l}-1.04 \\
(0.81)\end{array}$ & (dropped) \\
\hline Steinbrück ${ }^{\mathrm{b}}$ & $\begin{array}{c}16.62 * * * \\
(0.77)\end{array}$ & $\begin{array}{c}2.80 * * * \\
(0.42)\end{array}$ & $\begin{array}{c}16.13 * * * \\
(0.77)\end{array}$ & $\begin{array}{c}2.80 * * * \\
(0.42)\end{array}$ \\
\hline $\begin{array}{l}\text { Neither Merkel nor } \\
\text { Steinbrück }{ }^{b}\end{array}$ & $\begin{array}{l}2.81 * * \\
(0.99)\end{array}$ & $\begin{array}{c}1.85^{* * * *} \\
(0.48)\end{array}$ & $\begin{array}{c}3.37 * * * \\
(0.97)\end{array}$ & $\begin{array}{c}1.84 * * * \\
(0.49)\end{array}$ \\
\hline Rile self-placement & $\begin{array}{l}-0.36+ \\
(0.19)\end{array}$ & $\begin{array}{c}-0.33 * * * \\
(0.09)\end{array}$ & $\begin{array}{l}-0.47 * \\
(0.22)\end{array}$ & $\begin{array}{c}-0.33 * * * \\
(0.09)\end{array}$ \\
\hline INTERCEPT & $\begin{array}{c}0.42 \\
(1.31) \\
\end{array}$ & $\begin{array}{l}1.50^{*} \\
(0.60)\end{array}$ & $\begin{array}{c}0.85 \\
(1.40)\end{array}$ & $\begin{array}{l}1.49^{*} \\
(0.60)\end{array}$ \\
\hline \multicolumn{5}{|l|}{$\begin{array}{l}\text { Individual-specific: } \\
\text { FDP vote }\end{array}$} \\
\hline Post-election survey & $\begin{array}{l}-0.42 \\
(1.34)\end{array}$ & $\begin{array}{l}-0.41 \\
(0.35)\end{array}$ & $\begin{array}{l}-0.29 \\
(1.26)\end{array}$ & $\begin{array}{l}-0.41 \\
(0.35)\end{array}$ \\
\hline System knowledge & $\begin{array}{l}-1.12 \\
(0.83)\end{array}$ & $\begin{array}{c}-0.92 * * \\
(0.31)\end{array}$ & $\begin{array}{l}-1.08 \\
(0.82)\end{array}$ & $\begin{array}{c}-0.92^{* *} \\
(0.31)\end{array}$ \\
\hline Former USSR ${ }^{a}$ & $\begin{array}{c}-16.13 * * * \\
(0.69)\end{array}$ & (dropped) & $\begin{array}{c}-15.92 * * * \\
(0.67)\end{array}$ & (dropped) \\
\hline Poland $^{\text {a }}$ & $\begin{array}{l}1.78+ \\
(1.07)\end{array}$ & (dropped) & $\begin{array}{l}1.73+ \\
(1.04)\end{array}$ & (dropped) \\
\hline First generation & $\begin{array}{l}-0.70 \\
(0.88)\end{array}$ & (dropped) & $\begin{array}{l}-0.82 \\
(0.85)\end{array}$ & (dropped) \\
\hline Steinbrück ${ }^{b}$ & $\begin{array}{c}16.77 * * * \\
(1.48)\end{array}$ & $\begin{array}{c}1.76^{* *} \\
(0.64)\end{array}$ & $\begin{array}{c}16.35 * * * \\
(1.37)\end{array}$ & $\begin{array}{c}1.76^{* *} \\
(0.64)\end{array}$ \\
\hline $\begin{array}{l}\text { Neither Merkel nor } \\
\text { Steinbrück }{ }^{\mathrm{b}}\end{array}$ & $\begin{array}{l}2.61^{*} \\
(1.23)\end{array}$ & $\begin{array}{l}-0.63 \\
(0.92)\end{array}$ & $\begin{array}{l}2.66^{*} \\
(1.18)\end{array}$ & $\begin{array}{l}-0.63 \\
(0.93)\end{array}$ \\
\hline Rile self-placement & $\begin{array}{c}0.33 \\
(0.29)\end{array}$ & $\begin{array}{c}0.09 \\
(0.10)\end{array}$ & $\begin{array}{c}0.33 \\
(0.27)\end{array}$ & $\begin{array}{c}0.09 \\
(0.10)\end{array}$ \\
\hline INTERCEPT & -2.68 & -0.34 & -2.57 & -0.34 \\
\hline
\end{tabular}




\begin{tabular}{lcccc} 
& $(2.10)$ & $(0.71)$ & $(1.99)$ & $(0.71)$ \\
\hline $\mathrm{N}$ (alternatives) & 1235 & 10930 & 1235 & 10930 \\
$\mathrm{~N}$ (cases) & 247 & 2154 & 247 & 2154 \\
Bic & 641.21 & 2511.47 & 633.59 & 2520.71 \\
\hline
\end{tabular}

Note: $+\mathrm{p}<0.10,{ }^{*} \mathrm{p}<0.05,{ }^{* *} \mathrm{p}<0.01, * * * \mathrm{p}<0.001$; robust standard errors, clustered on constituencies, are shown in parentheses; models estimated on weighted data to compensate for an oversampling of East German regions and to make the data more representative of the socio-structural outlook of the German voting age population (weight variable in the GLES 2013 dataset: w_ipfges_2); ${ }^{a}$ reference category is remaining immigrant groups; ${ }^{b}$ reference group is Merkel 
Table A5: Robustness alternative-specific mixed multinomial logit models (random effects)

\begin{tabular}{lcc}
\hline & $\begin{array}{c}\text { Model 1: } \\
\text { Immigrants } \\
\text { PR votes }\end{array}$ & $\begin{array}{c}\text { Model 2: Natives' } \\
\text { PR votes }\end{array}$ \\
\hline $\begin{array}{l}\text { Alternative-specific } \\
\text { equation: }\end{array}$ & & \\
Candidate awareness & $1.73^{* *}$ & $0.90^{* * *}$ \\
& $(0.54)$ & $(0.15)$ \\
Party identification & $0.75^{* * *}$ & $0.65^{* * *}$ \\
& $(0.16)$ & $(0.05)$ \\
SMD vote & $0.64+$ & $1.43^{* * *}$ \\
& $(0.34)$ & $(0.12)$ \\
CIO list share & 0.97 & -0.41 \\
& $(1.59)$ & $(0.49)$ \\
CIO & $1.51^{* *}$ & -0.46 \\
& $(0.49)$ & $(0.36)$ \\
\hline sd(CIO) & 0.00 & 1.33 \\
& $(0.00)$ & $(0.49)$ \\
\hline
\end{tabular}

SPD vote

\begin{tabular}{|c|c|c|}
\hline Post-election survey & $\begin{array}{c}0.18 \\
(0.74)\end{array}$ & $\begin{array}{c}0.12 \\
(0.26)\end{array}$ \\
\hline System knowledge & $\begin{array}{c}0.37 \\
(0.71)\end{array}$ & $\begin{array}{l}-0.54^{*} \\
(0.26)\end{array}$ \\
\hline Former USSR $^{a}$ & $\begin{array}{l}-0.62 \\
(0.92)\end{array}$ & (dropped) \\
\hline Poland $^{\text {a }}$ & $\begin{array}{c}0.77 \\
(0.86)\end{array}$ & (dropped) \\
\hline First generation & $\begin{array}{c}0.41 \\
(0.85)\end{array}$ & (dropped) \\
\hline Steinbrück ${ }^{\mathrm{b}}$ & $\begin{array}{c}20.50 \\
(12.48)\end{array}$ & $\begin{array}{c}3.04 * * * \\
(0.45)\end{array}$ \\
\hline $\begin{array}{l}\text { Neither Merkel nor } \\
\text { Steinbrück }\end{array}$ & $\begin{array}{c}3.17^{* * *} \\
(0.86)\end{array}$ & $\begin{array}{c}2.11 * * * \\
(0.51)\end{array}$ \\
\hline Rile self-placement & $\begin{array}{l}-0.43^{*} \\
(0.21)\end{array}$ & $\begin{array}{c}-0.23^{* *} \\
(0.08)\end{array}$ \\
\hline INTERCEPT & $\begin{array}{c}0.30 \\
(1.47)\end{array}$ & $\begin{array}{c}0.67 \\
(0.49)\end{array}$ \\
\hline \multicolumn{3}{|c|}{$\begin{array}{l}\text { Individual-specific: The } \\
\text { Left vote }\end{array}$} \\
\hline Post-election survey & $\begin{array}{c}0.33 \\
(0.85)\end{array}$ & $\begin{array}{l}-0.07 \\
(0.28)\end{array}$ \\
\hline System knowledge & $\begin{array}{c}1.08 \\
(0.81)\end{array}$ & $\begin{array}{c}-0.76^{* *} \\
(0.27)\end{array}$ \\
\hline Former USSR $^{a}$ & $\begin{array}{c}-1.70+ \\
(1.01)\end{array}$ & (dropped) \\
\hline Poland $^{\text {a }}$ & $\begin{array}{l}1.45 \\
(0.88)\end{array}$ & (dropped) \\
\hline
\end{tabular}




\begin{tabular}{lcc} 
First generation & 0.22 & (dropped) \\
& $(0.81)$ & \\
Steinbrück $^{\mathrm{b}}$ & 19.55 & $2.76^{* * *}$ \\
& $(12.18)$ & $(0.48)$ \\
Neither Merkel nor & $4.63^{* * *}$ & $2.71^{* * *}$ \\
Steinbrück & $(0.95)$ & $(0.45)$ \\
Rile self-placement & $-0.84^{*}$ & $-0.80^{* * *}$ \\
& $(0.33)$ & $(0.09)$ \\
INTERCEPT & 1.36 & $3.38^{* * *}$ \\
& $(1.87)$ & $(0.55)$ \\
\hline
\end{tabular}

\section{Individual-specific:}

\section{Greens vote}

\begin{tabular}{|c|c|c|}
\hline Post-election survey & $\begin{array}{c}0.13 \\
(0.77)\end{array}$ & $\begin{array}{c}0.08 \\
(0.32)\end{array}$ \\
\hline System knowledge & $\begin{array}{l}1.29 \\
(0.80)\end{array}$ & $\begin{array}{l}-0.23 \\
(0.28)\end{array}$ \\
\hline Former USSR $^{\mathrm{a}}$ & $\begin{array}{l}-1.41 \\
(0.95)\end{array}$ & (dropped) \\
\hline Poland ${ }^{\mathrm{a}}$ & $\begin{array}{c}0.56 \\
(0.93)\end{array}$ & (dropped) \\
\hline First generation & $\begin{array}{l}-0.86 \\
(0.77)\end{array}$ & (dropped) \\
\hline Steinbrück ${ }^{b}$ & $\begin{array}{c}20.25 \\
(12.32)\end{array}$ & $\begin{array}{c}2.81 * * * \\
(0.43)\end{array}$ \\
\hline $\begin{array}{l}\text { Neither Merkel nor } \\
\text { Steinbrück }{ }^{\mathrm{b}}\end{array}$ & $\begin{array}{l}2.83 * * \\
(0.99)\end{array}$ & $\begin{array}{c}1.84 * * * \\
(0.49)\end{array}$ \\
\hline Rile self-placement & $\begin{array}{l}-0.34+ \\
(0.19)\end{array}$ & $\begin{array}{c}-0.34 * * * \\
(0.09)\end{array}$ \\
\hline INTERCEPT & $\begin{array}{c}0.40 \\
(1.32)\end{array}$ & $\begin{array}{l}1.58 * * \\
(0.59)\end{array}$ \\
\hline $\begin{array}{l}\text { Individual-specific: } \\
\text { FDP vote }\end{array}$ & & \\
\hline Post-election survey & $\begin{array}{l}-0.34 \\
(1.34)\end{array}$ & $\begin{array}{l}-0.44 \\
(0.35)\end{array}$ \\
\hline System knowledge & $\begin{array}{l}-1.14 \\
(0.84)\end{array}$ & $\begin{array}{c}-0.95 * * \\
(0.31)\end{array}$ \\
\hline Former USSR $^{a}$ & $\begin{array}{c}-19.55^{* * *} \\
(0.70)\end{array}$ & (dropped) \\
\hline Poland $^{\mathrm{a}}$ & $\begin{array}{l}1.78+ \\
(1.07)\end{array}$ & (dropped) \\
\hline First generation & $\begin{array}{l}-0.69 \\
(0.88)\end{array}$ & (dropped) \\
\hline Steinbrück ${ }^{\mathrm{b}}$ & $\begin{array}{l}20.28+ \\
(11.92)\end{array}$ & $\begin{array}{l}1.72 * * \\
(0.65)\end{array}$ \\
\hline $\begin{array}{l}\text { Neither Merkel nor } \\
\text { Steinbrück }{ }^{\mathrm{b}}\end{array}$ & $\begin{array}{l}2.58 * \\
(1.21)\end{array}$ & $\begin{array}{l}-0.67 \\
(0.94)\end{array}$ \\
\hline Rile self-placement & $\begin{array}{c}0.35 \\
(0.28)\end{array}$ & $\begin{array}{c}0.07 \\
(0.10)\end{array}$ \\
\hline INTERCEPT & -2.84 & -0.20 \\
\hline
\end{tabular}


(2.04)

$(0.73)$

\begin{tabular}{lcc}
\hline $\mathrm{N}$ (alternatives) & 1235 & 10930 \\
$\mathrm{~N}$ (cases) & 247 & 2154 \\
$\mathrm{Bic}$ & 634.68 & 2514.19 \\
\hline Note $+\mathrm{p}<0.10 * \mathrm{p}<0.05, * * \mathrm{p}<0.01, * * *$ & $\mathrm{p}<0.001 ;$
\end{tabular}

Note: $+p<0.10, * p<0.05,{ }^{* *} p<0.01,{ }^{* * *} p<0.001$; robust standard errors, clustered on constituencies, are shown in parentheses; models estimated on weighted data to compensate for an oversampling of East German regions and to make the data more representative of the socio-structural outlook of the German voting age population (weight variable in the GLES 2013 dataset: w_ipfges_2); ${ }^{\text {a }}$ reference category is remaining immigrant groups; ${ }^{b}$ reference group is Merkel 


\section{Aufsatz 2}

Geese, L., \& Schacht, D. (2019). The more concentrated, the better represented? The geographical concentration of immigrants and their descriptive representation in the German mixed-member system. International Political Science Review, 40(5), 643-658. https://doi.org/10.1177/0192512118796263

Das folgende Manuskript ist der Post-print des Aufsatzes, der von der Redaktion der Fachzeitschrift International Political Science Review (ISSN: 0192-5121) zur Publikation angenommen wurde. 
The more concentrated, the better represented? The geographical concentration of immigrants and their descriptive representation in the German mixed-member system

\title{
Lucas Geese
}

University of Bamberg, Germany

\section{Diana Schacht}

German Institute for Economic Research (DIW Berlin), Germany

\begin{abstract}
Does the geographical concentration of ethnic minorities influence their descriptive representation in closed-list systems? Counterintuitive to the idea that single-member district electoral rules are necessary for minorities' geographical representation, we argue that, in closed-list systems, parties are incentivized to allocate promising list positions to those minority candidates who are rooted in geographical areas where minorities concentrate. Empirically, we provide a case study of the list positions of dual candidates of immigrant-origin running in the German mixed-member system in 2013. Results show a relationship between the list positions of candidates of immigrant-origin and geographical concentrations of immigrant-origin residents.
\end{abstract}

\section{Keywords}

Descriptive representation, mixed-member systems, immigrant-origin candidates, geographical concentrations, ethnic minorities, closed-list systems

\section{Corresponding author:}

Lucas Geese, Faculty for Social Sciences, Economics, and Business

Administration, University of Bamberg, Feldkirchenstr. 21, 96052 Bamberg,

Germany.

Email: lucas.geese@uni-bamberg.de 


\section{Introduction}

Investigating the descriptive representation of ethnic minorities is a fundamental topic in political science. The representation of ethnic minorities' interests, their political participation, and trust in democratic institutions, hinges on minorities' adequate numerical (or descriptive) representation in decision-making assemblies (Mansbridge, 1999). Since proportional representation (PR) systems perform better than singlemember district (SMD) systems in producing proportionality between parties' vote and seat shares, they are often said to be more conducive to minority representation (Ruedin, 2009). However, scholarship demonstrates that the distinction between the two types of systems is not clear-cut. A key factor to be considered is the settlement patterns of minorities. That is, SMD systems can be more beneficial to geographically concentrated minorities than closed-list PR systems, while geographically dispersed minorities may find it easier to access the parliamentary ranks through closed-list PR systems (Bloemraad, 2013; Bochsler, 2011; Dancygier, 2014; Ruedin, 2009).

Evidence favouring the moderating effect of minorities' geographical concentrations is also provided by studies conducted in mixed-member (MM) systems (Donovan, 2007; Friedman, 2005; Kostadinova, 2007; Moser, 2008; Schönwälder, 2012; Wüst, 2014; Zollinger and Bochsler, 2012). Since MM systems create parliaments with a fixed share of seats elected under SMD and remaining seats allocated by PR (Shugart and Wattenberg, 2003), this line of research mainly seeks to make 'controlled' comparisons between levels of minority representation in the (closed-list) PR and in the SMD tier.

However, extant research in MM systems considers the geographical representation of minorities as a decisive factor in the SMD tier, while surprisingly little is known about the consequences of minorities' settlement patterns in the closed-list PR tier of 
MM systems. Consequently, we ask in this paper whether minorities' geographical concentration influences descriptive representation in the closed-list PR tier of MM systems. ${ }^{1}$ Pursuing this question provides an interesting research puzzle. From the perspective of formal electoral rules, minorities' geographical concentration may not be consequential in the PR tier because closed-list PR electoral rules lack the kind of geographical representation institutionalised in SMD systems (Latner and McGann, 2005). Conversely, minorities' geographical concentration could be a decisive factor for their descriptive representation in the closed-list PR tier of MM systems due to contamination effects between the two electoral tiers or due to informal recruitment rules requiring candidates to possess and maintain involvement in local politics. If candidates have such local attachments when running in the PR tier of MM systems, parties may have incentives to allocate promising list positions to minority candidates who are rooted in the geographical areas where minorities concentrate.

This article, we believe, is the first to pursue this research question. Moreover, by outlining a novel theoretical understanding of minorities' geographical representation, we make a theoretical contribution to the literature. Empirically, we provide a case study of list placements of dual candidates of immigrant origin running in the 18th German Bundestag elections held in 2013. Implemented in 1949, the German MM system has been in place continuously since then and is, therefore, the oldest existing MM system, which has, as a prototype, inspired other MM system designs globally in their basic, although not necessarily in all, features (Manow, 2015: 1-8). As such, the German electoral system may provide insights relevant for minority representation in other MM and closed-list PR systems, or for constitutional designers who plan to implement a German-style system. 
Empirical results provide evidence that the list positions of dual candidates of immigrant-origin are positively related to geographical concentrations of immigrantorigin residents. This suggests that, in addition to formal electoral rules, other factors can affect geographical patterns of minority representation. Thus, our knowledge about the interplay of electoral rules, minorities' geographical representation, and their descriptive representation is far from complete, urging future research to investigate other determinants of local minority representation than formal electoral rules.

\section{Electoral rules and the link between minorities' local concentration and their descriptive representation}

The geographical representation of ethnic minorities is widely considered to be an important feature of SMD electoral rules for two main reasons. First, when the electoral territory is carved up into several SMDs, geographically concentrated minorities can become electorally powerful in a number of districts (or even the local majority) so that minority candidates are more likely to be electorally successful in these districts (Bochsler, 2010, 2011; Dancygier, 2014; Trounstine and Valdini, 2008: 555). Second, minorities' geographical concentration is also supposed to strengthen minority candidates' grassroots support within parties (Dancygier, 2014; Garbaye, 2000: 300; Laurence and Maxwell, 2012: 27). Dense networks of ethnic associations foster minorities' political participation and increase their local power resources at the grassroots level (Fennema and Tillie, 1999). Thus, minorities have greater opportunities to request that local party selectorates choose a local minority politician as a candidate in the upcoming district election (Dancygier, 2014: 236; Garbaye, 2000; Laurence and Maxwell, 2012: 21-29; Norris and Lovenduski, 1995: 143-165). However, if minorities are geographically dispersed under SMD electoral rules, then minorities may lose their local voting power and minority aspirants cannot rely on similar levels of local 
grassroots support (Bochsler, 2010, 2011; Dancygier, 2014; Trounstine and Valdini, 2008). In other words, previous work suggests that the consequences of SMD electoral rules are contingent on minorities' geographical concentration.

In a similar vein, empirical research in MM systems mainly considers minorities' geographical concentration to be a decisive factor for descriptive representation in the SMD tier. Existing research in Eastern European MM systems suggests that, due to minorities' geographical concentration, the SMD tier provides often for similar or even better levels of minorities' descriptive representation compared to the $\mathrm{PR}$ tier (Friedman, 2005; Kostadinova, 2007). Other works suggest that the SMD tier may often be more conducive to minorities' descriptive representation since smaller districts are more likely to be ethnically homogenous, thus giving minorities more leverage to elect group members (Moser, 2008; Zollinger and Bochsler, 2012). Similarly, analysts of immigrant-minority representation in Germany ascribe lower levels of descriptive representation in the SMD tier to a pattern of modest geographical concentrations of immigrant groups (Donovan, 2007: 473; Schönwälder, 2012: 70-74).

Under closed-list PR electoral rules, whether in the PR tier of MM systems or in 'pure' PR systems, minorities' geographical concentration is not typically considered a driver of their descriptive representation. That may be because closed-list PR rules are typically envisioned to provide little leverage for geographical representation patterns (Latner and McGann, 2005). Moreover, closed-list PR rules are commonly thought to differ from SMD rules in that they put the electoral fate of minority candidates in the hands of national party elites rather than in those of local minority voters and/or activists. Regardless of minorities' settlement patterns, party elites are assumed to follow strategies of ticket-balancing reflecting the sociodemographic outlook of the overall electorate for the purpose of garnering votes from minority voters and to avoid 
negative publicity about a potentially undiversified party list (Dancygier, 2014: 328; Laurence and Maxwell, 2012: 15-20; Norris and Lovenduski, 1995: 195; Valdini, 2012: 741; Zollinger and Bochsler, 2012: 615). Accordingly, there may be no relationship between the list position of minority candidates in the PR tier of a MM system and the local concentration of minority groups. Thus, it is not surprising that there is, as far as we know, a lack of research on the impact of the geographical concentration of ethnic minorities on their descriptive representation in the closed-list PR tier of MM systems as well as in closed-list PR systems.

\section{Minorities' geographical representation in the PR tier of MM systems}

Minorities' geographical concentration and their descriptive representation in the closed-list PR tier of MM systems may be more strongly related with each other than previous scholarship on minority representation in MM systems suggests. First, candidates in the PR tier may be locally connected due to contamination effects between the SMD and the PR tier. Indeed, literature largely unrecognised by scholarship on minority representation in MM systems suggests that the existence of two electoral tiers provides parties, candidates, and voters with new avenues for strategic behaviour. Bochsler, for example, demonstrates that, in compensatory MM systems, larger parties can make (illegitimate) deals with other parties and voters for the purpose of bypassing the compensatory mechanism and increasing their overall seat shares (Bochsler, 2012). Since the pioneering work of Herron and Nishikawa (2001), proponents of cross-tier contamination argue similarly that the behaviour of parties and voters differs strongly in MM systems from that of parties and voters in 'pure' SMD or PR systems.

Contamination posits that voters reward parties with PR votes in response to the personal appeal of SMD candidates; that is, personal votes cast in the SMD tier spill 
over into votes for the party in the PR tier (Ferrara et al., 2005: 65-79; Hainmueller and Kern, 2008; Herron and Nishikawa, 2001; Manow, 2015: 61-85). Consequently, parties have vote-seeking incentives to nominate candidates to as many SMD districts as possible, even if they have no chance of winning the SMD race (Ferrara et al., 2005: 37; Herron and Nishikawa, 2001: 69-70). For this reason, it is common practice to nominate candidates in SMDs and in party lists simultaneously, so-called dual candidates $^{2}$ (Crisp, 2007; Ferrara et al., 2005; Hennl, 2014; Hennl and Kaiser, 2008; Manow, 2015: 161). In many MM systems, parties typically maximise their numbers of dual candidates by making the selection of candidates in the SMD tier a requirement for realistic list positions in the PR tier (Manow, 2015: 161). As local selection in the SMD tier is often considered a pre-condition for selection in realistic list positions in the closed-list PR tier, most viable list candidates may, therefore, be locally connected.

Second, party list candidates could be locally rooted due to informal recruitment requirements to maintain connections to local politics. In many closed-list PR and MM systems, political aspirants are required to gather political experience in local government, councils and/or party organisations before they are considered viable party list candidates. Even as elected legislators, they often keep their local mandates and offices in addition to their seats in the national parliament, thus effectively maintaining local political attachments. Evidence of such requirements is reported for many closed list systems, for instance, in Germany (Wessels, 1997), Israel (Hazan, 1999), Italy (Russo, 2011), the Netherlands (Leijenaar and Niemöller, 1997), and Portugal (Fernandes et al., 2017). For this reason, it is plausible to assume that grassroots support from local concentrations of ethnic minorities is often an inherent part of legislative recruitment in the closed-list PR tier of MM systems. Grassroots support ensures candidates' local selection in SMDs (if contamination is the driving mechanism), or 
their access to local party/government mandates (if local mandate requirement is the driving mechanism), if not both.

Given such local attachments, parties seeking minority votes may have an incentive to provide minority candidates with better list positions if they originate from areas with concentrations of minority voters. By allocating realistic list positions to local minority representatives, parties can signal to minority voters that it takes the issue of minority representation seriously. According to Krauss and colleagues (2012), local voters are aware of the list rankings that local candidates occupy in the PR tier of MM systems. Voters know 'that a high ranking... is a signal that the candidate is favored by the party' (p. 750) and influences 'the likelihood that the candidate will be elected of the list tier' (p. 751). In other words, parties' nomination decisions in the closed-list PR tier affect how voters evaluate local candidates and their parties. Thus, it is rational for minority voters to cast a vote for the local minority candidate's party list as it increases the likelihood that the minority candidate is elected off the list, and minority voters can simultaneously reward the party for providing for local descriptive representation. Anticipating this behaviour, party elites have then the opportunity to mobilise local minority voters by manipulating candidates' list rankings. Indeed, as every additional vote has the potential to increase a party's seat share under rules of proportional representation, parties' vote-seeking incentives to consider locally concentrated voting groups should be high.

This argumentation highlights the special role of local minority candidates for local minority mobilisation. Of course, in theory, the election of the candidate could simply be supported by voting for the party list regardless of whether the candidate is local or not. However, local candidates may be more visible to voters than other candidates on the list. As such, they could be utilised by their parties as important local campaigning 
resources. As local candidates, they could mobilise the minority vote by canvassing ethnic community groups, knocking on doors of minority voters, meeting them face-toface in the pedestrian area or by giving interviews to local media. In accordance, Gschwend and Zittel (2015) argue that local candidates establish important voting cues in MM systems because they are in close proximity to the voter and actually appear on the ballot paper in MM systems. Moreover, after the election, parties could assign the task of constituency casework to the (then elected) minority legislator for the purpose of nurturing, if not extending, the local minority voting base. For these reasons, party elites who seek the minority vote may have an incentive to place minority candidates higher on their party lists, if these candidates are from local areas where minority groups concentrate.

Thus, we hypothesise that minority candidates receive better list positions in the $P R$ tier if they are from areas of high minority concentration.

\section{Case selection, data and variables}

To study empirically the relationship between minorities' geographical concentrations and their descriptive representation in the closed-list PR tier of MM systems, we provide a case study of the 2013 German Bundestag elections focussing on the recruitment of immigrant-origin candidates in the PR tier. Germany's long-standing history of immigration and the increasing ethnic diversity of the electorate, combined with a stark underrepresentation of immigrant-origin citizens in the Bundestag make Germany a very relevant case for the study of immigrant-minority representation. Due to its prototypical relevance, Germany's MM proportional system is also widely considered a prime example for studying the consequences of MM systems for political representation (Manow, 2015: 1-8). 
Voters can cast a candidate vote in a single-member district race (SMD tier) as well as a party vote for a closed list of candidates in 16 multi-seat districts (PR tier). The system is further compensatory in that the PR tier determines the number of parliamentary seats that parties ultimately receive. Moreover, like in most MM systems, dual candidacy is an important institutional feature of the system. All main parties decide party list nominations after nominations have been decided at the local level in order to ensure priority for constituency candidates to receive the best positions in the list, while remaining (mainly unwinnable) list slots are filled with list-only candidates (Manow, 2015: 161; Reiser, 2014: 59). In the 2013 Bundestag election, for example, out of a total of 332 elected candidates in the PR tier, 306 , or $92 \%$, were elected as dual candidates, while the percentage of successful candidates who ran only on a list amounted to only $8 \%$ of all PR tier legislators.

Thus, the fact that almost all viable party list candidates are dual candidates provides us with the practical advantage of being able to link individual list candidates unambiguously to a specific local area. Consequently, we collected data on all 1138 dual candidates running in the 2013 election for one of the five most relevant political parties (CDU/CSU, SPD, FDP, The Left, and Greens), omitting the selective groups of district only $(n=355)$ and list only candidates $(n=538)$ from the sample. However, the seemingly advantage of analysing only dual candidates' list position has important consequences for disentangling the underlying assumed mechanisms. We explain this in greater detail in the discussion.

The dataset was compiled with the help of Parlamentwatch e.V., a registered German charity (www.abgeordnetenwatch.de), which records detailed background information on all constituency candidates. The organisation kindly provided the respective data, which we complemented with official data from the Federal Electoral 
Commissioner (Bundeswahlleiter, 2013) and sources described in the following paragraphs.

Given that candidate selection predetermines candidates' electoral prospects when closed party lists are in use (Hazan and Rahat, 2010: 13), we focus on candidates' list positions as a dependent variable. Thereby, it is instrumental to include successful as well as unsuccessful candidates in our analysis, because relying just on elected representatives (successful candidates) does not allow us to gain insights into the recruitment decisions taken before the election (Dancygier, 2014). To operationalize the dependent variable, we follow Hazan and Rahat, for whom a list position is viable if it can be considered 'winnable before the elections... [such that] there is a need for a clear-cut and fixed delineation of the "realistic" from the "unrealistic" (Hazan and Rahat, 2010: 14).

To capture parties' assessment of how promising each list position is, we build on Hennl and Kaisers' work on women's representation in Germany. In their study, the authors incorporate a survey with the executive committees of the regional party associations asking for the criteria they use for estimating safe list positions. The responses suggest that parties rely mainly on survey data in combination with previous election results. Moreover, since the authors also asked for the exact number of estimated safe list positions, they find that the average of list mandates won in the previous and in the analysed election is a good indicator of parties' anticipation of safe list positions (Hennl and Kaiser, 2008: 327). In line with this, we consider a candidates' list position as promising if it is smaller or equal to the average of list mandates that the party won in the previous and in the analysed election. Nonetheless, the last promising list position is of course less promising than those high on the list, while the position just below the defined delineation line can be considered as better than the last position 
on the entire list. We consider the significance of these differences by subtracting a candidate's individual list position from the last promising position on a list, thus producing a continuous measure of candidates' relative list positions. A value of ' 0 ' denotes the last 'promising' position on a respective list, while positive (negative) values refer to positions above (below) the threshold, while also indicating each position's numerical distance to the threshold.

Following previous research on immigrant-minority representation in Germany (Donovan, 2007; Schönwälder, 2012; Wüst, 2014), we define and code candidates in our dataset as being of immigrant origin if they were born (a) outside the Federal Republic of Germany's present territory with foreign nationality at birth; (b) in Germany but with foreign nationality at birth (first generation); or (c) with German nationality and at least one parent of foreign nationality at birth (second generation). For the actual coding of dual candidates' immigrant backgrounds, we draw mainly on the information published by 'Mediendienst Integration' (Mediendienst Integration 2013) and other publicly available sources, such as personal websites, party websites, Wikipedia entries, and media interviews. In this way, we are able to identify 73 candidates of immigrant-origin, which amounts to $6.4 \%$ of all candidates in this dataset (captured in the dichotomous variable $\mathrm{CIO}$ ).

To measure the size of the immigrant-origin electorate at the constituency-level, we rely on the percentage of foreign nationals in the population $(\% F N)$. It ranges from 1 to 28 percent, with an average of 9 percent. Obviously, this indicator can only approximate the immigrant-origin electorate, because foreign nationals, by definition, do not have the right to vote in national elections. Nevertheless, given that citizens of immigrant origin and foreign nationals tend to reside in the same neighbourhoods, $\% \mathrm{FN}$ is a reasonable approximation of a constituency's immigrant-origin electorate. Based on 
census data at the level of administrative districts (Kreise), in 2013 the correlation between the two indicators was $\mathrm{r}=0.78$ (Wüst 2014: 14). Moreover, another important limitation is that relying on this indicator does not allow us to distinguish between the population shares of different immigrant groups at the district-level. We return to this issue in the discussion.

A set of control variables is supposed to account for variations influencing candidates' relative list positions. At the level of regional multi-member districts, we control for district magnitude, accounting for the fact that party lists tend to be longer in districts of higher magnitude. At the party level, we include five dummy variables, one for each political party (CDU/CSU, SPD, Greens, The Left, and FDP). At the constituency level, we include voter turnout as well as parties'vote shares in the previous election ( $\mathrm{t}-1)$ to control for the possibility that parties reward more politically active and supportive constituencies with a 'list' legislator in parliament (Manow, 2015: 78).

At the level of individual candidates, we also consider sociodemographic differences that may make candidates more attractive to party list selectors, including whether or not candidates are female and have a university degree (tertiary education) as well as candidates' age to account for the possibility that party list selectors prefer candidates who are better educated or are in more advanced career stages (Norris and Lovenduski, 1995: 113-15). Lastly, given that incumbency status is an important informal nomination rule in Germany (Reiser, 2014: 59), we also control for whether a candidate is a 'list' or 'district' incumbent or neither.

Table 1 provides descriptive statistics of the variables described. Our dependent variable ranges from -50 to 18 with an average of -7 , showing that most dual candidates do not occupy a promising list position. Overall, 304 out of 1138 dual candidates 
occupy relative list positions greater or equal to zero, which seems a reasonable estimate when compared to the 306 dual candidates that were actually elected in the PR tier in 2013.

Table 1. Descriptive statistics

\begin{tabular}{llllll}
\hline & $\mathrm{N}$ & Mean & Min & Max & SD \\
\hline Relative list position & 1138 & -6.94 & -50 & 18 & 11.29 \\
\% FN & 1138 & 9.10 & 1.01 & 28.37 & 5.48 \\
CIO & 1138 & 0.06 & 0 & 1 & 0.25 \\
District magnitude & 1138 & 36.53 & 4 & 74 & 22.93 \\
CDU/CSU & 1138 & 0.21 & 0 & 1 & 0.41 \\
FDP & 1138 & 0.23 & 0 & 1 & 0.42 \\
Greens & 1138 & 0.19 & 0 & 1 & 0.39 \\
The Left & 1138 & 0.12 & 0 & 1 & 0.33 \\
SPD & 1138 & 0.25 & 0 & 1 & 0.43 \\
Voter turnout (t-1) & 1138 & 71.52 & 58.93 & 79.79 & 3.88 \\
Party vote share (t-1) & 1138 & 19.89 & 3.78 & 49.47 & 9.65 \\
Female & 1138 & 0.34 & 0 & 1 & 0.47 \\
Age & 1138 & 47.77 & 19 & 78 & 10.82 \\
Tertiary education & 1121 & 0.79 & 0 & 1 & 0.41 \\
Not incumbent & 1138 & 0.61 & 0 & 1 & 0.49 \\
List incumbent & 1138 & 0.23 & 0 & 1 & 0.42 \\
SMD incumbent & 1138 & 0.16 & 0 & 1 & 0.37 \\
\hline
\end{tabular}

\section{Data analysis and results}

Using candidates' relative list positions as a dependent variable allows us to compare candidates' electoral prospects across party lists and multi-member districts. However, the empirical analysis must take into account that our operationalisation of relative list positions only constitutes a 'limited' measure (Long, 1997: 187) of candidates' electoral prospects in the PR tier. To illustrate this point, consider the example of two candidates occupying relative list positions of, say, -50 and -40 . Although the candidate occupying the latter position is ten positions higher up on the list, her chance of obtaining a seat in parliament is equally bad when compared to the other candidate (the likelihood of getting elected is close to $0 \%$ for both candidates) ${ }^{3}$, such that this difference in list positions should not be relevant to voters, candidates, or parties. It follows that the effect of minorities' geographical concentration described in our article does not apply to differences in relative list positions below a certain list position threshold. For this 
reason, it is instrumental to censor observations falling below this threshold when examining our dependent variable in a regression analysis (Long, 1997: 188). When the dependent variable is limited in this way, Tobit regression models produce more accurate parameter estimates than OLS models and, hence, are preferred (Long, 1997: 189). Consequently, we employ Tobit models that left-censor the sample for relative list positions smaller than -20 , thus censoring candidates who have less than a $3 \%$ chance of getting elected in the PR tier. ${ }^{4}$ Results presented do not vary greatly when changing the censoring threshold (see supplementary material). To take into account the clustering of the data inside multi-member districts, all regression coefficients are estimated with robust standard errors clustered on the 16 German Länder.

Table 2 presents results of six Tobit models determining candidates' relative list positions. The first model examines the impact of local immigrant shares, candidates' immigrant backgrounds, and an interaction term ${ }^{5}$ of these variables on the dependent variable without considering the impact of the control variables. By including the interaction term, we intend to test whether dual candidates of immigrant-origin occupy relatively higher list positions in the German PR tier when they originate from areas with a higher local foreigner share or whether the two indicators are statistically unrelated to each other.

The statistically significant positive effect of the interaction term suggests that a $10 \%$ increase in local immigrant shares (about two standard deviations) improves the relative list placement of a candidate of immigrant origin by one position $((-0.15+0.26)$ * $10=1.1)$, while the insignificant effect of the constituent term $(\% \mathrm{FN})$ indicates that the local immigrant share has a slightly negative, nonsignificant effect on the relative list position of other candidates. The negative effect of the constituent term suggests 
that for every immigrant-origin candidate who moves upward on the list, another candidate moves down, thus producing a small negative coefficient. 
Table 2. Tobit regression models

\begin{tabular}{|c|c|c|c|c|c|c|}
\hline & Model 1 & Model 2 & Model 3 & Model 4 & Model 5 & Model 6 \\
\hline \multicolumn{7}{|l|}{ Independent variables } \\
\hline \multirow[t]{2}{*}{$\% \mathrm{FN}^{\mathrm{a}}$} & -0.15 & 0.02 & -0.14 & -0.15 & -0.21 & -0.07 \\
\hline & $(0.12)$ & $(0.08)$ & $(0.11)$ & $(0.12)$ & $(0.11)$ & $(0.08)$ \\
\hline \multirow[t]{2}{*}{$\mathrm{CIO}$} & 2.31 & 2.31 & 1.32 & 2.30 & $2.87 *$ & 1.81 \\
\hline & $(1.76)$ & (1.61) & (1.49) & (1.59) & $(1.37)$ & $(1.12)$ \\
\hline \multirow[t]{2}{*}{$\mathrm{CIO} * \% \mathrm{FN}$} & $0.26 * *$ & $0.16^{*}$ & $0.23 *$ & $0.26 * *$ & $0.24 * *$ & $0.21 *$ \\
\hline & $(0.08)$ & $(0.08)$ & $(0.11)$ & $(0.09)$ & $(0.08)$ & $(0.09)$ \\
\hline \multicolumn{7}{|l|}{$\begin{array}{l}\text { Multi-member district } \\
\text { level controls }\end{array}$} \\
\hline \multirow{2}{*}{ District magnitude $^{\mathrm{a}}$} & & $-0.12 * * *$ & & & & $-0.11 * * *$ \\
\hline & & $(0.02)$ & & & & $(0.02)$ \\
\hline \multirow[t]{2}{*}{ FDP } & & & $-3.22 *$ & & & $-4.76^{*}$ \\
\hline & & & $(1.26)$ & & & $(2.42)$ \\
\hline \multirow[t]{2}{*}{ Grüne } & & & 0.39 & & & -3.13 \\
\hline & & & $(1.35)$ & & & $(1.70)$ \\
\hline \multirow[t]{2}{*}{ Linke } & & & $4.39^{* * *}$ & & & 0.53 \\
\hline & & & $(0.84)$ & & & $(1.26)$ \\
\hline \multirow[t]{2}{*}{ SPD } & & & $2.12^{*}$ & & & 1.14 \\
\hline & & & $(1.05)$ & & & $(1.60)$ \\
\hline \multicolumn{7}{|l|}{$\begin{array}{l}\text { Constituency-level } \\
\text { controls }^{a}\end{array}$} \\
\hline \multirow[t]{2}{*}{ Voter turnout (t-1) } & & & & 0.01 & & 0.13 \\
\hline & & & & $(0.10)$ & & $(0.07)$ \\
\hline \multirow{2}{*}{$\begin{array}{l}\text { Party vote share (t- } \\
\text { 1) }\end{array}$} & & & & -0.00 & & $-0.16^{* * *}$ \\
\hline & & & & $(0.05)$ & & $(0.03)$ \\
\hline \multicolumn{7}{|l|}{$\begin{array}{l}\text { Candidate-level } \\
\text { controls }\end{array}$} \\
\hline \multirow[t]{2}{*}{ Female candidate } & & & & & $3.31^{* * *}$ & $2.49 * * *$ \\
\hline & & & & & $(0.58)$ & $(0.49)$ \\
\hline \multirow[t]{2}{*}{ Tertiary education } & & & & & $1.39^{*}$ & $1.79 * *$ \\
\hline & & & & & $(0.58)$ & $(0.57)$ \\
\hline \multirow[t]{2}{*}{$\mathrm{Age}^{\mathrm{a}}$} & & & & & -0.02 & $-0.04 *$ \\
\hline & & & & & $(0.01)$ & $(0.02)$ \\
\hline \multirow[t]{2}{*}{$\mathrm{C}$ List incumbent ${ }^{\mathrm{c}}$} & & & & & $11.46^{* * *}$ & $11.46 * * *$ \\
\hline & & & & & $(2.32)$ & (2.34) \\
\hline \multirow[t]{2}{*}{ C SMD incumbent ${ }^{c}$} & & & & & $4.55^{* * *}$ & $5.06^{* * *}$ \\
\hline & & & & & $(1.14)$ & $(1.32)$ \\
\hline \multirow[t]{2}{*}{ Constant } & $-6.42 * * *$ & $-6.54 * * *$ & $-6.78 * * *$ & $-6.42 * * *$ & $-11.96 * * *$ & $-10.65 * * *$ \\
\hline & $(1.13)$ & $(0.47)$ & $(0.94)$ & (1.11) & $(2.04)$ & $(1.17)$ \\
\hline \multirow[t]{2}{*}{ Sigma } & $9.61 * * *$ & $9.33^{* * *}$ & $9.32 * * *$ & $9.61 * * *$ & $7.99 * * *$ & $7.41 * * *$ \\
\hline & $(1.56)$ & $(1.56)$ & $(1.50)$ & (1.56) & $(1.12)$ & $(1.05)$ \\
\hline $\mathrm{N}$ & 1138 & 1138 & 1138 & 1138 & 1121 & 1121 \\
\hline Censored N & 144 & 144 & 144 & 144 & 141 & 141 \\
\hline Log-likelihood & -3835.26 & -3793.49 & -3799.81 & -3835.24 & -3592.52 & -3501.08 \\
\hline Nagelkerke $\mathrm{R}^{2}$ & 0.01 & 0.08 & 0.07 & 0.01 & 0.30 & 0.40 \\
\hline
\end{tabular}

Note: Tobit regression models with left-censoring threshold at relative list position of -20. Table entries are unstandardised coefficients with robust standard errors clustered on 16 multi-member districts in parantheses. ${ }^{a}$ Variables are centered at their global mean; ${ }^{\mathrm{b}}$ 'CDU/CSU' is the reference category; ${ }^{\mathrm{c}}$ 'Not incumbent' is the reference category; ${ }^{*} \mathrm{p}<0.05,{ }^{*} \mathrm{p}<0.01, * * * \mathrm{p}<0.001$. 
In Models 2 to 5, we add control variables depending on their level of measurement to the regression model. Most control variables work as expected and, as the models' fit statistics reveal (indicated by higher values for Log-likelihood and Nagelkerkes' $\mathrm{R}^{2}$ ), differences in district magnitude, party affiliations, and candidate-level characteristics feature strongest in the explanation of candidates' relative list positions. Candidate-level variables have particularly strong explanatory power, mostly due to the incumbency variable, thus indicating that incumbent PR legislators are strongly advantaged on the list. Nevertheless, besides these influences, in all models the previously described effect of the interaction term remains robust, that is, indicating a statistically significant and positive effect of the local percentage of foreign nationals on the relative list placements of immigrant-origin candidates. Even in Model 6, where we add all control variables to the model, the relationship remains stable and the effect size substantial. Increasing the local percentage of foreign nationals in this model by $7 \%$ (about one and a third standard deviations) improves the relative list placement of candidates of immigrantorigin by one position $((-0.07+0.21) * 7=0.98)$.

Further elaborations of our data suggest that the statistical association is mainly driven by the three parties on the political left (SPD, Greens, The Left). However, this does not come as a surprise given the well-known left-wing bias of immigrant-minority representation in Western European democracies (e.g. Bloemraad, 2013: 664). We provide additional empirical material and discussions on this issue in the supplementary material to this paper. Taken together, results do not change considerably, when model 6 is replicated for left-wing parties only, because left-wing parties are a priori more likely to select immigrant-origin candidates in any case, and because the distribution of foreigner shares is strongly biased towards smaller values for the few immigrant-origin candidates who run for right-wing parties. 
Figure 1. Marginal effects of $\mathrm{CIO}$ on relative list position depending on local share of foreign nationals with $95 \%$ confidence intervals

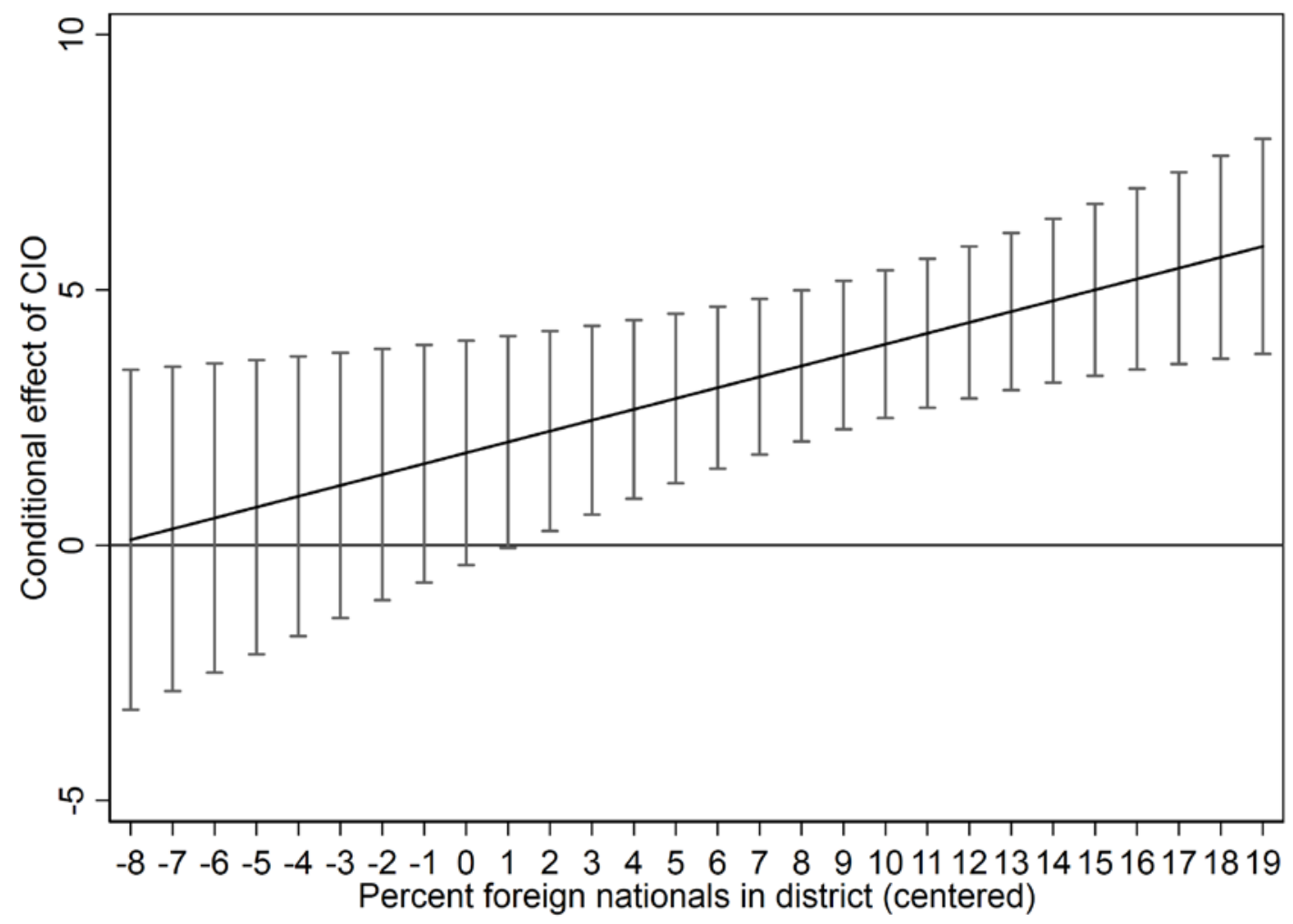

Note: Based on Model 7 reported in Table 2. Remaining variables are hold at their means

Figure 1 visualises the finding from Model 6 in a marginal effect plot showing how the effect of $\mathrm{CIO}$ on the realistic list position changes conditional on the local immigrant share (centred). The upward slope suggests that the impact of CIO on the dependent variable increases as the local share of foreign nationals increases. From the $95 \%$ confidence intervals, it can be inferred that the positive effect of CIO becomes significant when the share of foreign nationals exceeds the average foreigner share by at least $2 \%$. Taken together, these results indicate that the list positions of dual candidates of immigrant-origin are positively related to geographical concentrations of immigrantorigin residents. Thus, we consider our hypothesis as confirmed. For robustness checks, please see the supplementary material. 


\section{Discussion and conclusion}

In this paper, we pursue the question of whether geographical patterns of minority representation exist in the PR tier of the German MM system. A review of previous research does not suggest the existence of a link between minorities' geographical concentrations and their descriptive representation in the closed-list PR tier of MM systems. Subsequently, two arguments of why candidates may have, in many cases, local roots despite their nomination on a closed party list are proposed. First, party list candidates run in many $\mathrm{MM}$ systems simultaneously as SMD candidates due to contamination effects between the SMD and PR tier. Second, under formal closed-list PR electoral rules, candidates and legislators are often involved in local politics, for example as local councillors or party officials. Given these local attachments, party elites may have incentives to allocate better list positions to minority candidates who are rooted in areas of high minority density in order to attract the local minority vote. In line with this argumentation, the presented empirical evidence shows that dual candidates of immigrant-origin received better party list positions preceding the 2013 Bundestag elections when they were selected to run simultaneously as district candidates in local constituencies where many citizens of immigrant-origin reside.

Despite this, our study has several limitations. First, a major concern is the unavailability of detailed district-level sociodemographic data. Due to the non-existence of such data, we have to rely on the local share of foreign nationals as a proxy for the immigrant-origin electorate in candidates' home areas. However, in an ideal world, we would not only have a direct measure of our target group at the local level, but we would also be able to distinguish between the shares of different immigrant groups. Unfortunately, we are unable to do so because there is no district-level sociodemographic data available. For this reason, our analysis relies on the implicit 
assumption that immigrant-origin voters prefer any immigrant-origin candidate over a native candidate, even if the immigrant-origin candidate has a different national background than the voter.

Although this is not ideal, it can be argued that when minority voters are unable to select a representative with reliable descriptive characteristics, they nevertheless prefer pseudo-descriptive representatives, whose characteristics mimic descriptive representation (Mansbridge, 1999: 645). Such an understanding is also supported by empirical research on immigrants' voting behaviour. This literature suggests that disadvantaged immigrant groups tend to see their own social and economic disadvantages as linked to the disadvantages of immigrants more generally (Sanders et al., 2014). For this reason, most immigrant voters tend to vote for immigrant-origin candidates, irrespective of whether the candidate has a matching or different national background, because voters perceive immigrant-origin candidates to be more trustworthy than native candidates in representing the interests of immigrant groups (Bergh and Bjorklund, 2011; Teney et al., 2010; Zingher and Farrer, 2016). In addition, it can be argued that relying on the foreigner share should make for a tough test of our hypothesised link as it should bias the coefficient of the interaction term downward in case there was no voting link between voters and candidates of different immigrant origin.

Second, as the German MM system operates in a PR tier with several multimember districts, the composition of party lists may be targeted to the regional sociodemographic profile. This relationship might feed into the link between minorities' local concentration and their descriptive representation. In other words, it is possible that list positions of immigrant-origin candidates are to some extent affected by the minority population shares of multi-member districts. Although this potential effect is 
accounted for in our empirical models through the consideration of robust standard errors clustered at the level of regions (multi-member districts), the question remains whether our finding is specific to the German system or whether it applies also to MM systems operating in national PR tiers. Although we cannot answer this question, it can be argued that minor changes in $\mathrm{MM}$ electoral system design have occurred in $\mathrm{MM}$ systems in the past and may happen in the future; for example changing from a national to a regionally differentiated PR tier, or vice versa. Therefore, even if our finding is specific to the German-style system, electoral system reformers may want to consider it when evaluating potential changes to the territorial organisation of the PR tier of MM systems.

A third limitation is that our single-country study does not allow us to distinguish the consequences of contamination-induced dual candidacy requirements from consequences of candidates' other involvements in local politics. Unfortunately, including a control group of list-only candidates as a means to separate these effects is not feasible. List-only candidates are a priori very unlikely to receive a promising list position; therefore, they are too selective a group to be compared to dual candidates in Germany.

Although our study leaves open a number of questions, it makes a major contribution to the study of electoral systems and minority representation as it is the first to shed light on the link between minorities' local concentration and their descriptive representation in the closed-list PR tier of MM systems. As such, it makes plausible arguments for why this link should be there and provides first empirical evidence from a country that is particularly relevant with regard to immigrant-minority representation. Thus, the present contribution is an important reference point for future studies of minorities' descriptive representation in MM and closed-list PR systems. A 
crucial point is the possibility of geographical representation in the PR tier as a consequence of contamination effects. In fact, confirmation of contaminated minority representation flowing from the SMD to the PR tier would have important implications for previous scholarship conducted in MM systems. This line of research relies exclusively on the assumption of independent electoral tiers. In this regard, although our article cannot ultimately prove contamination effects, at least it casts doubts regarding the validity of the assumption of independence. Thus, we urge future researchers to keep in mind that it is not necessarily appropriate to assume independent electoral tiers when studying minorities' descriptive representation in MM systems. This article narrows crucial gaps in previous scholarship on minority representation in $\mathrm{MM}$ and closed-list PR systems, while outlining avenues for future research.

To test the generalisability of the finding, future studies should examine the extent to which it travels to other MM as well as to 'pure' closed-list PR systems. First, future research may develop and test hypotheses regarding the consequences of different institutional variations within the broader category of MM systems. Although the German system is widely recognised to be the prototype MM system, guiding implementation in other countries (Manow, 2015: 1-8), these systems do not resemble the German one in every detail. We already noted that other systems might operate in a nationwide rather than in several regional multi-member districts. MM systems can also differ in other aspects, for example as to whether the PR tier is compensatory or not, and as to whether dual candidacy ${ }^{6}$ is allowed or not (Krauss et al., 2012; Shugart and Wattenberg, 2003). While we would not necessarily expect patterns of minorities' geographical representation in the PR tier to differ depending on such different MM system designs, only future empirical scrutiny will determine if the found geographical representation link also exists in these. 
Second, we need research on minorities' geographical representation in 'pure' closed-list PR systems as well as comparisons of this type of system with MM systems. Indeed, geographical patterns of minority representation may not be limited to the PR component of MM systems. Since candidates' involvement in local politics is a common feature of many 'pure' closed-list PR systems, similar effects may be found here. Moreover, by comparing MM with 'pure' closed-list PR systems, future research should be better able to disentangle effects of contamination from effects of candidates' local involvement that generally apply to closed-list PR electoral systems. Based on such a comparative research design, the contamination thesis would find strong corroboration if minorities' geographical representation is found to be a more decisive feature in mixed than in 'pure' systems. A finding of similar patterns of geographical representation in both types of systems would, on the other hand, strengthen the thesis that such representational patterns can be a feature of any closed-list system.

However, new databases are needed to allow the tracing of minority representation across different electoral systems and, ideally, even further down the 'ladder of recruitment' by incorporating the aspirants applying for candidacy in the analysis. Future research should build such databases and investigate whether, how, and which institutions of local representation matter to minorities' descriptive representation across a wide range of electoral systems. 


\section{Acknowledgements}

An earlier version of this article was presented at the 74th Annual MPSA Conference 2016 in Chicago. We thank Thomas Saalfeld, David Lublin, Henning Bergmann, Javier Martínez Cantó, Simon Fink, Margret Hornsteiner, Stefanie John, André Krouwel, Juan Rodríguez Teruel, Nadja Wehl, and four anonymous reviewers for helpful comments and suggestions. We also thank Adam Lederer and Joanna MacLeod for proofreading, and gratefully acknowledge the support of the Abgeordnentenwatch website's administrators (http://www.abgeordnetenwatch.de/), who kindly provided us with an extract of their database.

\section{Funding}

This work was supported by the German Research Foundation (DFG) [SA 2160/3-1].

\section{Supplementary material}

Supplementary material is available at journals.sagepub.com/home/ips. 


\section{Notes}

${ }^{1}$ Throughout this paper, the terms 'minority' and 'ethnic minority' are used interchangeably. Following previous research (Bochsler, 2010; Moser, 2008, p. 280; Ruedin, 2009), ethnic minority groups are in a numerical minority position and members of these groups self identify with each other due to common religion, language, cultural history, race, or national identity. Thus, the definition applies to minority groups with a long-standing history of residence in a country (autochthonous) as well as to immigrant minorities (allochthonous).

${ }^{2}$ Dual candidacy exists in many MM systems, among them Hungary, Italy (before 2005), Japan (lower chamber), Lithuania, New Zealand, Scotland, Wales (since 2016) and the German regional parliaments (see Crisp, 2007: 1462; Ferrara et al., 2005: 18; Hennl, 2014: 94; Krauss et al., 2012: 754). Exceptions extend to Japan (upper chamber), Thailand, Ukraine and Wales (before 2016).

${ }^{3}$ To illustrate this point statistically, we fit a bivariate probit regression model estimating the effect of relative list positions on the likelihood of getting elected off a party list in 2013 (see Table A1 in the supplementary material) and also visualised the relationship in a marginal effects plot (Figure A1 in the supplementary material). The graph shows a distinct 'floor effect' of our dependent variable: In other words, the relative list position between the lowest observed value of -50 and approximately -20 has literally no positive effect on a candidate's electoral prospects, while increasing the relative list positions between values of approximately -20 and 18 translates continuously into better electoral prospects.

${ }^{4}$ This election likelihood follows from the probit regression estimation shown in Table and Figure A1.

${ }^{5}$ The centring of $\% \mathrm{FN}$ at its mean value is supposed to handle multicollinearity between the interaction term and its constituent terms (see also supplementary material). 
${ }^{6}$ In order to examine whether the dual candidacy provision is prerequisite for contaminationinduced geographical representation in MM systems, future research may turn to cases that do not allow for dual candidacy. For example, it may be possible to study how the implementation of dual candidacy in Wales in 2016 influenced minority representation as compared to previous elections. Alternatively, future research may compare minority representation in Wales (before 2016) and Scotland, a MM system that allows dual candidacy. As both systems are subnational British electoral systems, this research design would resemble a most similar systems design.

\section{References}

Bergh, Johannes and Tor Bjorklund (2011). The Revival of Group Voting: Explaining the Voting Preferences of Immigrants in Norway. Political Studies 59(2): 308-327.

Bloemraad, Irene (2013). Accessing the Corridors of Power: Puzzles and Pathways to Understanding Minority Representation. West European Politics 36(3): 652-670.

Bochsler, Daniel (2010). Electoral rules and the representation of ethnic minorities in post-communist democracies. European Yearbook of Minority Issues 7: 153-180.

Bochsler, Daniel (2011). It is not how many votes you get, but also where you get them. Territorial determinants and institutional hurdles for the success of ethnic minority parties in post-communist countries. Acta Politica 46(3): 217-38.

Bochsler, Daniel (2012). A quasi-proportional electoral system ‘only for honest men'? The hidden potential for manipulating mixed compensatory electoral systems. International Political Science Review 33(4): 401-20.

Bundeswahlleiter (2013). Wahl zum 18. Deutschen Bundestag am 22. September 2013. Sonderheft: Die Wahlbewerber für die Wahl zum 18. Deutschen Bundestag 2013. Wiesbaden. 
Crisp, Brian F. (2007). Incentives in Mixed-Member Electoral Systems: General Election Laws, Candidate Selection Procedures, and Cameral Rules. Comparative Political Studies 40(12): 1460-1485.

Dancygier, Rafaela M. (2014). Electoral Rules or Electoral Leverage? Explaining Muslim Representation in England. World Politics 66(2): 229-263.

Donovan, Barbara (2007). 'Minority' Representation in Germany. German Politics 16(4): 455-480.

Fennema, Meindert and Jean Tillie (1999). Political Participation and political trust in Amsterdam: civic communities and ethnic networks. Journal of Ethnic and Migration Studies 25(4): 703-726.

Fernandes, Jorge M, Cristina Leston-Bandeira and Carsten Schwemmer (2017). Election proximity and representation focus in party-constrained environments. Party Politics (July 2016).

Ferrara, Federico, Eric S Herron and Misa Nishikawa (2005). Mixed Electoral Systems. Contamination and its Consequences. New York: Palgrave Macmillan.

Friedman, Eden (2005). Electoral System Design and Minority Representation in Slovakia and Macedonia. Ethnopolitics 4(4): 381-96.

Garbaye, Romain (2000). Ethnic minorities, cities and Institutions: A comparisonof the modes of management of ethnic diversity of a French and British city. In Ruud Koopmans and Paul Statham (eds), Challenging Immigration and Ethnic Relations Politics. Oxford: Oxford University Press.

Gschwend, Thomas and Thomas Zittel, (2015). Do constituency candidates matter in German Federal Elections? The personal vote as an interactive process. Electoral Studies 39: 338-349. 
Hainmueller, Jens and Holger L Kern (2008). Incumbency as a source of spillover effects in mixed electoral systems: Evidence from a regression-discontinuity design. Electoral Studies 27(2): 213-227.

Hazan, Reuven Y (1999). Constituency interests without constituencies: the geographical impact of candidate selection on party organization and legislative behavior in the 14th Israeli Knesset, 1996-99. Political Geography 18: 791-811.

Hazan, Reuven Y and Gideon Rahat (2010). Democracy within Parties: Candidate Selection Methods and Their Political Consequences. Oxford: OUP Oxford.

Hennl, Annika (2014). Intra-party dynamics in mixed-member electoral systems: How strategies of candidate selection impact parliamentary behaviour. Journal of Theoretical Politics 26(1): 93-116.

Hennl, Annika and André Kaiser (2008). Ticket-balancing in mixed-member proportional systems. Comparing sub-national elections in Germany. Electoral Studies 27(2): 321-336.

Herron, Eric S and Misa Nishikawa (2001). Contamination effects and the number of parties in mixed-superposition electoral systems. Electoral Studies 20(1): 63-86.

Kostadinova, Tatiana (2007). Ethnic and women's representation under mixed election systems. Electoral Studies 26: 418-31.

Krauss, Ellis, Kuniaki Nemoto and Robert Pekkanen (2012). Reverse Contamination: Burning and Building Bridges in Mixed-Member Systems. Comparative Political Studies 45(6): 747-773.

Latner, Michael and McGann, Anthony (2005). Geographical representation under proportional representation: The cases of Israel and the Netherlands. Electoral Studies 24(4): 709-734. 
Laurence, Jonathan and Rashaan Maxwel, (2012). Political Parties and Diversity in Western Europe. In Teri Givens and Rashaan Maxwell (eds), Immigrant Politics: Race and Representation in Western Europe. Boulder: Lynne Rienner Publishers. Leijenaar, Monique and Niemöller, Kees (1997). In Pippa Norris (ed.), Passages to power. Legislative recruitment in advanced democracies. Cambridge: Cambridge University Press.

Long, J Scott (1997). Regression Models for Categorical and Limited Dependent Variables. Thousand Oaks: Sage.

Manow, Philip (2015). Mixed Rules, Mixed Strategies. Candidates and Parties in Germany's Electoral System. Colchester: ECPR Press.

Mansbridge, Jane (1999). Should Blacks Represent Blacks and Women Represent Women? A Contingent 'Yes'. The Journal of Politics 61(3): 628-657.

Mediendienst Integration. (2013). Bundestagskandidaten mit Migrationshintergrund. Retrieved 9 March 2016, from https://mediendienstintegration.de/fileadmin/Dateien/Bundestagskandidaten_MDI.pdf

Moser, Robert G (2008). Electoral Systems and the Representation of Ethnic Minorities: Evidence from Russia. Comparative Politics 40(3): 273-292.

Norris, Pippa and Joni Lovenduski (1995). Political Recuitment: Gender, Race, and Class in the British Parliament. Cambridge: Cambridge University Press.

Reiser, Marion (2014). The universe of group representation in Germany: Analysing formal and informal party rules and quotas in the process of candidate selection. International Political Science Review 35(1): 55-66.

Ruedin, Didier (2009). Ethnic Group Representation in a Cross-National Comparison. The Journal of Legislative Studies 15(4): 335-354. 
Russo, Federico (2011). The Constituency as a Focus of Representation: Studying the Italian Case through the Analysis of Parliamentary Questions. The Journal of Legislative Studies 17(3): 290-301.

Sanders, David, Anthony F Heath, Stephen D Fisher and Maria Sobolewska (2014). The Calculus of Ethnic Minority Voting in Britain. Political Studies 62: 230-51.

Schönwälder, Karen (2012). Cautious Steps: Minority Representation in Germany. In Terri E Givens and Rashaan Maxwell (eds), Immigrant Politics: Race and Representation in Western Europe. Boulder: Lynne Rienner Publishers.

Shugart, Matthew S and Martin P Wattenberg (2003). Mixed-Member Electoral Systems: A Definition and Typology. In Matthew S Shugart and Martin P Wattenberg (eds), Mixed-Member Electoral Systems: The Best of Both Worlds? Oxford: Oxford University Press.

Teney, Celine, Dirk Jacobs, Andrea Rea and Pascal Delwit (2010). Ethnic voting in Brussels: Voting patterns among ethnic minorities in Brussels (Belgium) during the 2006 local elections. Acta Politica 45(3): 273-97.

Trounstine, Jessica L and Melody E Valdini (2008). The Context Matters: The Effect of Single Member vs At-Large Districts on City Council Diversity. American Journal of Political Science 68(4): 554-569.

Valdini, Melody E (2012). A deterrent to diversity: The conditional effect of electoral rules on the nomination of women candidates. Electoral Studies 31: 740-749.

Wessels, Bernhard (1997). Germany. In Pippa Norris (ed.), Passages to power. Legislative recruitment in advanced democracies. Cambridge: Cambridge University Press.

Wüst, Andreas M. (2014). Immigration into Politics: Immigrant-origin Candidates and 
Their Success in the 2013 Bundestag Election. German Politics \& Society 32(3): $1-15$.

Zingher, Joshua N and Benjamin Farrer (2016). The electoral effects of the descriptive representation of ethnic minority groups in Australia and the UK. Party Politics 22(6): 691-704.

Zollinger, Daniel and Daniel Bochsler (2012). Minority representation in a semidemocratic regime: the Georgian case. Democratization 19(4): 611-41.

\section{Author biographies}

Lucas Geese is a doctoral candidate in the Faculty for Social Sciences, Economics, and Business Administration at the University of Bamberg, Germany. His research interests focus on political institutions, electoral systems, and political representation. In his doctoral dissertation he studies the link between electoral systems and immigrantminorities' political representation in European parliamentary democracies.

Diana Schacht is a Research Associate at the German Institute for Economic Research (DIW Berlin). Her research focuses on processes of migration and integration, social networks, and quantitative methods. Her work appears in a number of journals including Ethnic and Racial Studies, Ethnicities, and the Kölner Zeitschrift für Soziologie und Sozialpsychologie. 


\section{Supplementary Material to paper}

"The more concentrated, the better represented? The geographical concentration of immigrants and their descriptive representation in the German mixed-member system" 
Table A1. Probit regression model to estimate impact of relative list positions on party list elections

\section{Probit regression model}
Relative list position
$0.10 * * *$
$(0.01)$

$\begin{array}{lc}\text { Constant } & -0.01 \\ & (0.05) \\ \mathrm{N} & 904 \\ \text { Log-likelihood } & -431.07 \\ \text { Nagelkerke's R } & 0.39\end{array}$
Note: Table entries are unstandardised coefficients with standard errors in parentheses; Candidates elected in
the SMD tier excluded, $* \mathrm{p}<0.05, * * \mathrm{p}<0.01, * * * \mathrm{p}<0.001$.

Figure A1. Predicted probabilities of party list election by relative list position

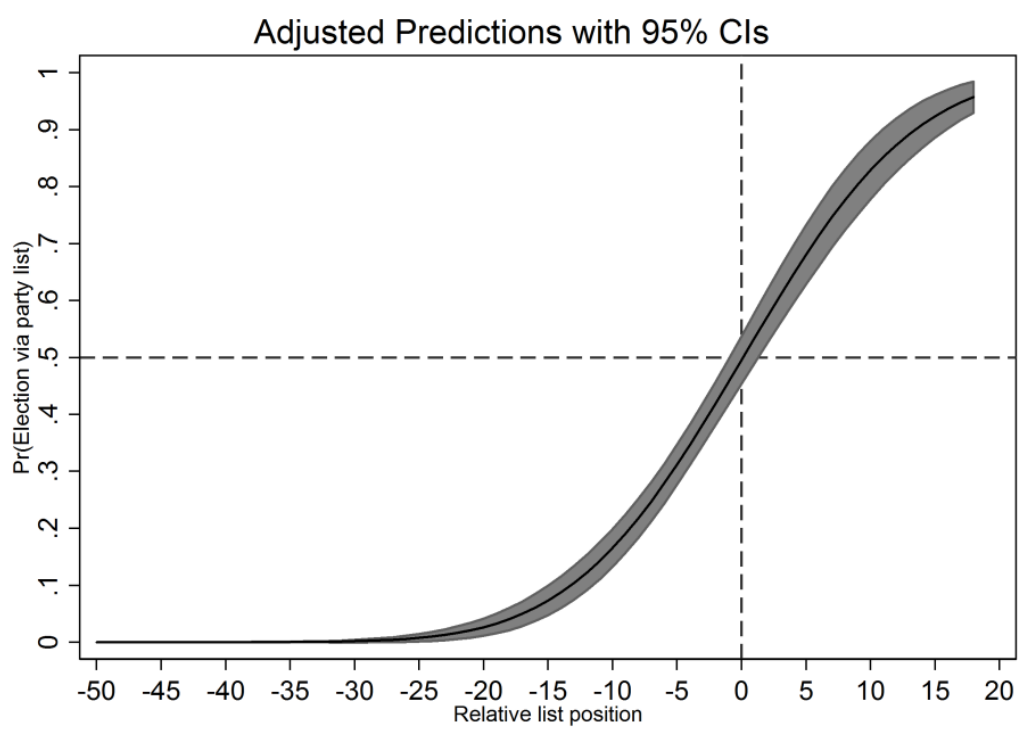

Note: Predicted probabilities of party list election by relative list position with $95 \%$ confidence intervals based on a bivariate probit model presented in Table A1. 
In this appendix, we provide a number of additional empirical materials. In order to demonstrate that $\% \mathrm{FN}$ is not highly truncated for candidates of immigrant-origin and, thus, not biasing the results of the interaction effect, Figure A2 presents the distribution of $\% \mathrm{FN}$ for candidates with and without immigrant origin separately in the form of histograms. Although candidates of immigrant-origin tend to run more often in constituencies with higher immigrant shares, the distribution for candidates of immigrant-origin is not worrisome in the sense of a highly skewed distribution.

Figure A2. Histograms of local shares of foreign nationals for candidates with and without immigrant-origin
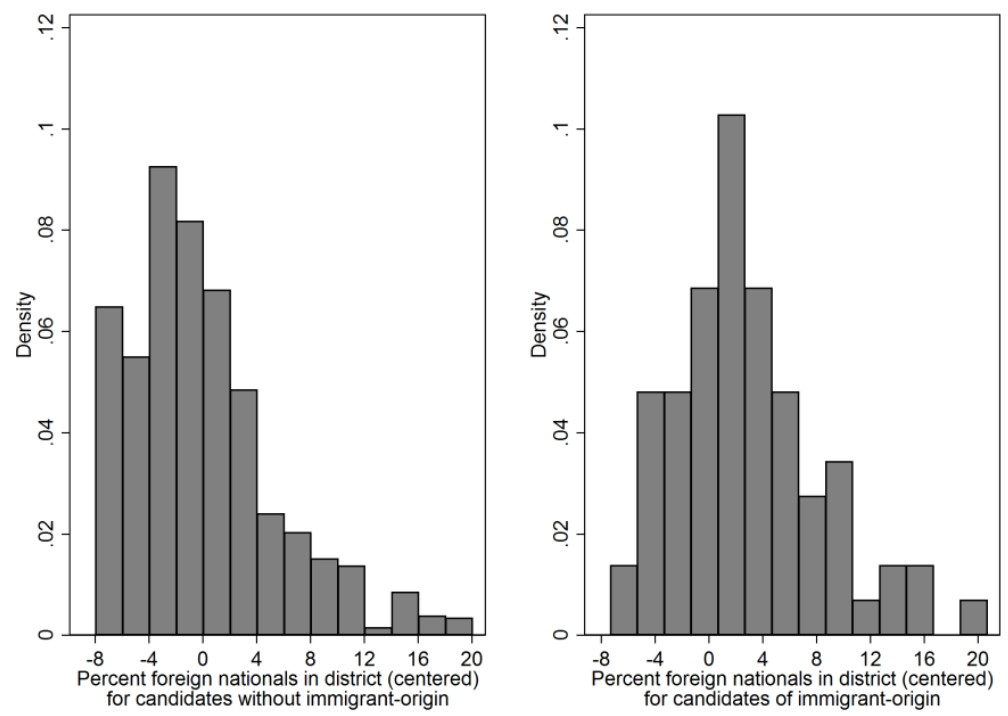

Note: own calculation based on data described in text

In table $A 2$, we replicate the main model (model 6 in table 2) with different censoring thresholds between -18 and -25 (models 1 to 7 ) and with no censoring (model 8) replicating the results of the main analysis. 
Table A2. Tobit regression models with varying left-censoring thresholds of the relative list position

\begin{tabular}{|c|c|c|c|c|c|c|c|c|}
\hline $\begin{array}{l}\text { Left- } \\
\text { censoring at }\end{array}$ & $\begin{array}{c}\text { Model } 1 \\
-18\end{array}$ & $\begin{array}{c}\text { Model } 2 \\
-19\end{array}$ & $\begin{array}{c}\text { Model } 3 \\
-21\end{array}$ & $\begin{array}{c}\text { Model } 4 \\
-22\end{array}$ & $\begin{array}{c}\text { Model } 5 \\
-23\end{array}$ & $\begin{array}{c}\text { Model } 6 \\
-24\end{array}$ & $\begin{array}{c}\text { Model } 7 \\
-25\end{array}$ & $\begin{array}{c}\text { Model } 8 \\
\text { None }\end{array}$ \\
\hline$\% \mathrm{FN}^{\mathrm{a}}$ & $\begin{array}{l}-0.07 \\
(0.07)\end{array}$ & $\begin{array}{l}-0.07 \\
(0.08)\end{array}$ & $\begin{array}{l}-0.07 \\
(0.08)\end{array}$ & $\begin{array}{l}-0.06 \\
(0.09)\end{array}$ & $\begin{array}{l}-0.06 \\
(0.09)\end{array}$ & $\begin{array}{l}-0.07 \\
(0.09)\end{array}$ & $\begin{array}{l}-0.07 \\
(0.09)\end{array}$ & $\begin{array}{l}-0.07 \\
(0.11)\end{array}$ \\
\hline $\mathrm{CIO}$ & $\begin{array}{l}1.73+ \\
(1.02)\end{array}$ & $\begin{array}{l}1.80+ \\
(1.09)\end{array}$ & $\begin{array}{l}1.87+ \\
(1.12)\end{array}$ & $\begin{array}{l}1.89+ \\
(1.14)\end{array}$ & $\begin{array}{c}1.90 \\
(1.16)\end{array}$ & $\begin{array}{c}1.91 \\
(1.19)\end{array}$ & $\begin{array}{c}1.90 \\
(1.20)\end{array}$ & $\begin{array}{l}2.18+ \\
(1.26)\end{array}$ \\
\hline $\mathrm{CIO} * \% \mathrm{FN}$ & $\begin{array}{l}0.21 * * \\
(0.08)\end{array}$ & $\begin{array}{l}0.21^{*} \\
(0.08)\end{array}$ & $\begin{array}{l}0.21^{*} \\
(0.08)\end{array}$ & $\begin{array}{l}0.21^{*} \\
(0.09)\end{array}$ & $\begin{array}{l}0.21 * \\
(0.09)\end{array}$ & $\begin{array}{l}0.21 * \\
(0.09)\end{array}$ & $\begin{array}{l}0.21 * \\
(0.09)\end{array}$ & $\begin{array}{c}0.16 \\
(0.12)\end{array}$ \\
\hline $\begin{array}{l}\text { District } \\
\text { magnitude }\end{array}$ & $\begin{array}{c}-0.10 * * * \\
(0.02)\end{array}$ & $\begin{array}{c}-0.10 * * * \\
(0.02)\end{array}$ & $\begin{array}{c}-0.11 * * * \\
(0.02)\end{array}$ & $\begin{array}{c}-0.12 * * * \\
(0.02)\end{array}$ & $\begin{array}{c}-0.12 * * * \\
(0.02)\end{array}$ & $\begin{array}{c}-0.12 * * * \\
(0.02)\end{array}$ & $\begin{array}{c}-0.12 * * * \\
(0.02)\end{array}$ & $\begin{array}{c}-0.15 * * * \\
(0.02)\end{array}$ \\
\hline $\mathrm{FDP}^{\mathrm{b}}$ & $\begin{array}{l}-4.43+ \\
(2.36)\end{array}$ & $\begin{array}{l}-4.60+ \\
(2.39)\end{array}$ & $\begin{array}{l}-4.97 * \\
(2.48)\end{array}$ & $\begin{array}{l}-5.12 * \\
(2.53)\end{array}$ & $\begin{array}{l}-5.25^{*} \\
(2.56)\end{array}$ & $\begin{array}{l}-5.40^{*} \\
(2.60)\end{array}$ & $\begin{array}{l}-5.51^{*} \\
(2.63)\end{array}$ & $\begin{array}{l}-6.62 * \\
(3.05)\end{array}$ \\
\hline Grüne $^{b}$ & $\begin{array}{l}-2.87+ \\
(1.67)\end{array}$ & $\begin{array}{l}-2.99+ \\
(1.68)\end{array}$ & $\begin{array}{l}-3.32+ \\
(1.76)\end{array}$ & $\begin{array}{l}-3.41+ \\
(1.78)\end{array}$ & $\begin{array}{l}-3.48+ \\
(1.79)\end{array}$ & $\begin{array}{l}-3.56^{*} \\
(1.81)\end{array}$ & $\begin{array}{l}-3.61 * \\
(1.80)\end{array}$ & $\begin{array}{l}-4.13 * \\
(1.94)\end{array}$ \\
\hline Linke $^{b}$ & $\begin{array}{c}0.63 \\
(1.26)\end{array}$ & $\begin{array}{c}0.59 \\
(1.27)\end{array}$ & $\begin{array}{c}0.45 \\
(1.31)\end{array}$ & $\begin{array}{c}0.41 \\
(1.33)\end{array}$ & $\begin{array}{c}0.38 \\
(1.33)\end{array}$ & $\begin{array}{c}0.33 \\
(1.35)\end{array}$ & $\begin{array}{c}0.30 \\
(1.35)\end{array}$ & $\begin{array}{l}-0.03 \\
(1.46)\end{array}$ \\
\hline $\mathrm{SPD}^{\mathrm{b}}$ & $\begin{array}{l}1.25 \\
(1.56)\end{array}$ & $\begin{array}{l}1.20 \\
(1.59)\end{array}$ & $\begin{array}{c}1.07 \\
(1.64)\end{array}$ & $\begin{array}{l}1.00 \\
(1.67)\end{array}$ & $\begin{array}{c}0.95 \\
(1.68)\end{array}$ & $\begin{array}{c}0.89 \\
(1.70)\end{array}$ & $\begin{array}{c}0.86 \\
(1.72)\end{array}$ & $\begin{array}{c}0.42 \\
(1.95)\end{array}$ \\
\hline $\begin{array}{l}\text { Voter turnout } \\
(\mathrm{t}-1)^{\mathrm{a}}\end{array}$ & $\begin{array}{c}0.11 \\
(0.07)\end{array}$ & $\begin{array}{l}0.12+ \\
(0.07)\end{array}$ & $\begin{array}{l}0.14+ \\
(0.07)\end{array}$ & $\begin{array}{l}0.14+ \\
(0.07)\end{array}$ & $\begin{array}{l}0.15^{*} \\
(0.07)\end{array}$ & $\begin{array}{l}0.15^{*} \\
(0.07)\end{array}$ & $\begin{array}{l}0.16^{*} \\
(0.08)\end{array}$ & $\begin{array}{l}0.22 * \\
(0.09)\end{array}$ \\
\hline $\begin{array}{l}\text { Party vote } \\
\text { share }(\mathrm{t}-1)^{\mathrm{a}}\end{array}$ & $\begin{array}{c}-0.15 * * * \\
(0.03)\end{array}$ & $\begin{array}{c}-0.16^{* * *} \\
(0.03)\end{array}$ & $\begin{array}{c}-0.16^{* * *} \\
(0.03)\end{array}$ & $\begin{array}{c}-0.16 * * * \\
(0.03)\end{array}$ & $\begin{array}{c}-0.17 * * * \\
(0.04)\end{array}$ & $\begin{array}{c}-0.17 * * * \\
(0.04)\end{array}$ & $\begin{array}{c}-0.17 * * * \\
(0.04)\end{array}$ & $\begin{array}{c}-0.18 * * * \\
(0.04)\end{array}$ \\
\hline $\begin{array}{l}\text { Female } \\
\text { candidate }\end{array}$ & $\begin{array}{c}2.44 * * * \\
(0.49)\end{array}$ & $\begin{array}{c}2.45 * * * \\
(0.48)\end{array}$ & $\begin{array}{c}2.52 * * * \\
(0.50)\end{array}$ & $\begin{array}{c}2.58 * * * \\
(0.52)\end{array}$ & $\begin{array}{c}2.59 * * * \\
(0.51)\end{array}$ & $\begin{array}{c}2.66 * * * \\
(0.54)\end{array}$ & $\begin{array}{c}2.67 * * * \\
(0.53)\end{array}$ & $\begin{array}{c}3.01 * * * \\
(0.65)\end{array}$ \\
\hline $\begin{array}{l}\text { Tertiary } \\
\text { education }\end{array}$ & $\begin{array}{l}1.68 * * \\
(0.52)\end{array}$ & $\begin{array}{l}1.72 * * \\
(0.53)\end{array}$ & $\begin{array}{l}1.78 * * \\
(0.56)\end{array}$ & $\begin{array}{l}1.77 * * \\
(0.54)\end{array}$ & $\begin{array}{l}1.85 * * \\
(0.57)\end{array}$ & $\begin{array}{l}1.87 * * \\
(0.58)\end{array}$ & $\begin{array}{l}1.84 * * \\
(0.56)\end{array}$ & $\begin{array}{c}1.78 * * * \\
(0.48)\end{array}$ \\
\hline $\mathrm{Age}^{\mathrm{a}}$ & $\begin{array}{l}-0.04^{*} \\
(0.02)\end{array}$ & $\begin{array}{l}-0.04^{*} \\
(0.02)\end{array}$ & $\begin{array}{l}-0.04 * \\
(0.02)\end{array}$ & $\begin{array}{c}-0.04 * * \\
(0.02)\end{array}$ & $\begin{array}{c}-0.05 * * \\
(0.02)\end{array}$ & $\begin{array}{c}-0.05^{* *} \\
(0.02)\end{array}$ & $\begin{array}{c}-0.05 * * \\
(0.02)\end{array}$ & $\begin{array}{c}-0.06 * * * \\
(0.02)\end{array}$ \\
\hline $\begin{array}{l}\text { C List } \\
\text { incumbent }^{c}\end{array}$ & $\begin{array}{c}11.3 * * * \\
(2.26)\end{array}$ & $\begin{array}{c}11.4 * * * \\
(2.30)\end{array}$ & $\begin{array}{c}11.6 * * * \\
(2.37)\end{array}$ & $\begin{array}{c}11.6 * * * \\
(2.40)\end{array}$ & $\begin{array}{c}11.7 * * * \\
(2.42)\end{array}$ & $\begin{array}{c}11.8 * * * \\
(2.46)\end{array}$ & $\begin{array}{c}11.8 * * * \\
(2.48)\end{array}$ & $\begin{array}{c}12.4 * * * \\
(2.71)\end{array}$ \\
\hline $\begin{array}{l}\text { C SMD } \\
\text { incumbent }^{c}\end{array}$ & $\begin{array}{c}5.03 * * * \\
(1.28)\end{array}$ & $\begin{array}{c}5.04 * * * \\
(1.30)\end{array}$ & $\begin{array}{c}5.00 * * * \\
(1.34)\end{array}$ & $\begin{array}{c}4.98 * * * \\
(1.33)\end{array}$ & $\begin{array}{c}4.98 * * * \\
(1.30)\end{array}$ & $\begin{array}{c}4.96 * * * \\
(1.28)\end{array}$ & $\begin{array}{c}4.96 * * * \\
(1.28)\end{array}$ & $\begin{array}{c}4.86^{* * *} \\
(1.34)\end{array}$ \\
\hline Constant & $\begin{array}{c}-10.5 * * * \\
(1.17) \\
\end{array}$ & $\begin{array}{c}-10.6^{* * *} \\
(1.17) \\
\end{array}$ & $\begin{array}{c}-10.6^{* * * *} \\
(1.17) \\
\end{array}$ & $\begin{array}{c}-10.7 * * * \\
(1.19) \\
\end{array}$ & $\begin{array}{c}-10.7 * * * \\
(1.18)\end{array}$ & $\begin{array}{c}-10.8 * * * \\
(1.16) \\
\end{array}$ & $\begin{array}{c}-10.8 * * * \\
(1.16)\end{array}$ & $\begin{array}{c}-10.9 * * * \\
(1.29)\end{array}$ \\
\hline Sigma & $\begin{array}{c}7.20 * * * \\
(1.00)\end{array}$ & $\begin{array}{c}7.30 * * * \\
(1.02)\end{array}$ & $\begin{array}{c}7.52 * * * \\
(1.09)\end{array}$ & $\begin{array}{c}7.63 * * * \\
(1.11)\end{array}$ & $\begin{array}{c}7.73 * * * \\
(1.13)\end{array}$ & $\begin{array}{c}7.82 * * * \\
(1.15)\end{array}$ & $\begin{array}{c}7.90 * * * \\
(1.17)\end{array}$ & $\begin{array}{c}8.83 * * * \\
(1.38)\end{array}$ \\
\hline $\begin{array}{l}\mathrm{N} \\
\text { Censored N }\end{array}$ & 1121 & $\begin{array}{c}1121 \\
154\end{array}$ & 1121 & 1121 & 1121 & 1121 & $\begin{array}{c}1121 \\
92\end{array}$ & 1121 \\
\hline $\begin{array}{l}\text { Log- } \\
\text { likelihood }\end{array}$ & -3397.68 & -3453.59 & -3545.00 & -3585.36 & -3624.79 & -3661.44 & -3695.73 & -4032.63 \\
\hline $\begin{array}{l}\text { Nagelkerke } \\
\mathrm{R}^{2}\end{array}$ & 0.40 & 0.40 & 0.40 & 0.40 & 0.40 & 0.40 & 0.40 & 0.39 \\
\hline
\end{tabular}

Note: Table entries are unstandardised coefficients with robust standard errors clustered on 16 multi-

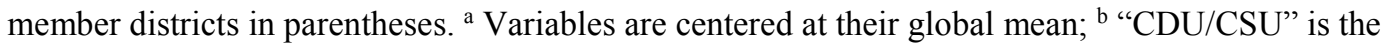
reference category; " "Not incumbent" is the reference category; $+\mathrm{p}<0.10, * \mathrm{p}<0.05, * * \mathrm{p}<0.01, * * *$ $\mathrm{p}<0.001$.

In table A3, we present further robustness checks.

- Since the variables CIO, \%FN $(r=0.13)$ and their interaction term are somewhat correlated with each other ( 0.45 and 0.28$)$, model 1 includes the main model without 
the interaction term, while models 2 and 3 include either $\mathrm{CIO}$ or $\% \mathrm{FN}$. The results do not differ substantially from the models presented in the paper.

- Model 4 presents the results of the main model when $\% \mathrm{FN}$ is not centred, showing that the interaction turns out to be positive and significant, while the two constituent terms do not provide much explanatory power. Moreover, the insignificance of the $\mathrm{CIO}$ variable in model 4 shows that multicollinearity issues seem to become more problematic when $\% \mathrm{FN}$ is not centred.

- In model 5 of table A3, we address potential concerns regarding the manipulation of our dependent variable by estimating the main model as an OLS regression with the dependent variable being candidates' actual list positions (first position $=1$, second position=2, ...). Since higher values indicate positions further down the list, we would expect a negative coefficient for the interaction term and, indeed, this is what we find.

- Model 6 addresses the concern that candidates' migration background may not be visible to voters. To tackle this concern, we generate a variable called visible $C I O$, that is, minority candidates who are visible based on their names or physical appearance. This shrinks the number of CIOs further $(n=53)$, which makes it more difficult to reach conventional levels of statistical significance in regression analysis. As seen in Model 6, the interaction effect between visible $\mathrm{CIO}$ and $\% \mathrm{FN}$ remains positive, but does not reach conventional levels of statistical significance $(\mathrm{p}=0.102)$.

- Model 7 replicates the main model, but only for parties of the left. The discussion of this model follows below Table A3. 
Table A3. Additional regression models

\begin{tabular}{|c|c|c|c|c|c|c|c|}
\hline & $\begin{array}{c}\text { Model 1: } \\
\text { Tobit } \\
\end{array}$ & $\begin{array}{c}\text { Model 2: } \\
\text { Tobit } \\
\end{array}$ & $\begin{array}{c}\text { Model 3: } \\
\text { Tobit } \\
\end{array}$ & $\begin{array}{c}\text { Model 4: } \\
\text { Tobit } \\
\end{array}$ & $\begin{array}{c}\text { Model 5: } \\
\text { OLS }^{d}\end{array}$ & $\begin{array}{c}\text { Model 6: } \\
\text { Tobit } \\
\end{array}$ & $\begin{array}{c}\text { Model 7: } \\
\text { Tobit } \\
\end{array}$ \\
\hline$\% \mathrm{FN}^{\mathrm{a}}$ & $\begin{array}{l}-0.05 \\
(0.08)\end{array}$ & & $\begin{array}{l}-0.04 \\
(0.08)\end{array}$ & & $\begin{array}{c}0.09 \\
(0.12)\end{array}$ & $\begin{array}{l}-0.07 \\
(0.08)\end{array}$ & $\begin{array}{l}-0.08 \\
(0.10)\end{array}$ \\
\hline $\mathrm{CIO}$ & $\begin{array}{l}2.35^{*} \\
(0.95)\end{array}$ & $\begin{array}{l}2.21 * * \\
(0.76)\end{array}$ & & $\begin{array}{l}-0.09 \\
(1.77)\end{array}$ & $\begin{array}{l}-2.07 \\
(1.30)\end{array}$ & & $\begin{array}{l}1.28 \\
(1.35)\end{array}$ \\
\hline $\mathrm{CIO} * \% \mathrm{FN}$ & & & & & $\begin{array}{l}-0.24^{*} \\
(0.11)\end{array}$ & & $\begin{array}{l}0.24^{*} \\
(0.12)\end{array}$ \\
\hline Visible CIO & & & & & & $\begin{array}{l}2.27 * \\
(0.96)\end{array}$ & \\
\hline $\begin{array}{l}\text { Visible CIO * } \\
\% \mathrm{FN}\end{array}$ & & & & & & $\begin{array}{c}0.17 \\
(0.10)\end{array}$ & \\
\hline $\begin{array}{l}\% \mathrm{FN} \text { (not } \\
\text { centered) } \\
\mathrm{CIO} * \% \mathrm{FN} \\
\text { (not centered) }\end{array}$ & & & & $\begin{array}{l}-0.07 \\
(0.08) \\
0.21 * \\
(0.09)\end{array}$ & & & \\
\hline $\begin{array}{l}\text { District } \\
\text { magnitude }\end{array}$ & $\begin{array}{c}-0.11 * * * \\
(0.02)\end{array}$ & $\begin{array}{c}-0.11 * * * \\
(0.01)\end{array}$ & $\begin{array}{c}-0.11 * * * \\
(0.02)\end{array}$ & $\begin{array}{c}-0.11 * * * \\
(0.02)\end{array}$ & $\begin{array}{c}0.34 * * * \\
(0.02)\end{array}$ & $\begin{array}{c}-0.11 * * * \\
(0.02)\end{array}$ & $\begin{array}{c}-0.08 * * * \\
(0.02)\end{array}$ \\
\hline $\mathrm{FDP}^{\mathrm{b}}$ & $\begin{array}{r}-4.68+ \\
(2.42)\end{array}$ & $\begin{array}{l}-4.66+ \\
(2.41)\end{array}$ & $\begin{array}{l}-4.78+ \\
(2.46)\end{array}$ & $\begin{array}{l}-4.76^{*} \\
(2.42)\end{array}$ & $\begin{array}{l}4.96^{*} \\
(2.07)\end{array}$ & $\begin{array}{l}-4.76+ \\
(2.44)\end{array}$ & \\
\hline Grüne $^{b}$ & $\begin{array}{l}-2.99+ \\
(1.70)\end{array}$ & $\begin{array}{l}-2.97+ \\
(1.70)\end{array}$ & $\begin{array}{l}-2.93+ \\
(1.70)\end{array}$ & $\begin{array}{l}-3.13+ \\
(1.70)\end{array}$ & $\begin{array}{l}4.76^{* *} \\
(1.37)\end{array}$ & $\begin{array}{l}-3.13+ \\
(1.73)\end{array}$ & \\
\hline Linke $^{b}$ & $\begin{array}{c}0.62 \\
(1.26)\end{array}$ & $\begin{array}{c}0.65 \\
(1.24)\end{array}$ & $\begin{array}{c}0.78 \\
(1.19)\end{array}$ & $\begin{array}{c}0.53 \\
(1.26)\end{array}$ & $\begin{array}{l}-0.51 \\
(1.51)\end{array}$ & $\begin{array}{c}0.55 \\
(1.25)\end{array}$ & $\begin{array}{c}3.74 * * * \\
(1.09)\end{array}$ \\
\hline $\mathrm{SPD}^{\mathrm{b}}$ & $\begin{array}{l}1.21 \\
(1.62)\end{array}$ & $\begin{array}{c}1.25 \\
(1.58)\end{array}$ & $\begin{array}{l}1.25 \\
(1.63)\end{array}$ & $\begin{array}{c}1.14 \\
(1.60)\end{array}$ & $\begin{array}{l}4.54 * * \\
(1.47)\end{array}$ & $\begin{array}{c}1.14 \\
(1.61)\end{array}$ & $\begin{array}{l}3.81 * * \\
(1.37)\end{array}$ \\
\hline $\begin{array}{l}\text { Voter turnout } \\
(\mathrm{t}-1)^{\mathrm{a}}\end{array}$ & $\begin{array}{l}0.13+ \\
(0.07)\end{array}$ & $\begin{array}{c}0.11 \\
(0.07)\end{array}$ & $\begin{array}{l}0.13+ \\
(0.07)\end{array}$ & $\begin{array}{l}0.13+ \\
(0.07)\end{array}$ & $\begin{array}{l}-0.21^{*} \\
(0.10)\end{array}$ & $\begin{array}{l}0.13+ \\
(0.07)\end{array}$ & $\begin{array}{c}0.10 \\
(0.06)\end{array}$ \\
\hline $\begin{array}{l}\text { Party vote share } \\
(\mathrm{t}-1)^{\mathrm{a}}\end{array}$ & $\begin{array}{c}-0.15 * * * \\
(0.03)\end{array}$ & $\begin{array}{c}-0.15 * * * \\
(0.03)\end{array}$ & $\begin{array}{c}-0.16 * * * \\
(0.03)\end{array}$ & $\begin{array}{c}-0.16 * * * \\
(0.03)\end{array}$ & $\begin{array}{l}0.21 * \\
(0.08)\end{array}$ & $\begin{array}{c}-0.16 * * * \\
(0.03)\end{array}$ & $\begin{array}{l}-0.07^{*} \\
(0.03)\end{array}$ \\
\hline $\begin{array}{l}\text { Female } \\
\text { candidate }\end{array}$ & $\begin{array}{c}2.46 * * * \\
(0.49)\end{array}$ & $\begin{array}{c}2.42 * * * \\
(0.48)\end{array}$ & $\begin{array}{c}2.39 * * * \\
(0.48)\end{array}$ & $\begin{array}{c}2.49 * * * \\
(0.49)\end{array}$ & $\begin{array}{c}-3.01 * * * \\
(0.63)\end{array}$ & $\begin{array}{c}2.48 * * * \\
(0.49)\end{array}$ & $\begin{array}{c}2.70 * * * \\
(0.74)\end{array}$ \\
\hline $\begin{array}{l}\text { Tertiary } \\
\text { education }\end{array}$ & $\begin{array}{l}1.81 * * \\
(0.57)\end{array}$ & $\begin{array}{l}1.77 * * \\
(0.54)\end{array}$ & $\begin{array}{l}1.76^{* *} \\
(0.56)\end{array}$ & $\begin{array}{l}1.79 * * \\
(0.57)\end{array}$ & $\begin{array}{c}-1.48 * * \\
(0.48)\end{array}$ & $\begin{array}{l}1.83 * * \\
(0.58)\end{array}$ & $\begin{array}{l}1.57^{*} \\
(0.62)\end{array}$ \\
\hline Age $^{a}$ & $\begin{array}{l}-0.04^{*} \\
(0.02)\end{array}$ & $\begin{array}{l}-0.04^{*} \\
(0.02)\end{array}$ & $\begin{array}{c}-0.05 * * \\
(0.02)\end{array}$ & $\begin{array}{l}-0.04 * \\
(0.02)\end{array}$ & $\begin{array}{l}0.05^{* *} \\
(0.01)\end{array}$ & $\begin{array}{c}-0.04 * * \\
(0.02)\end{array}$ & $\begin{array}{l}-0.02 \\
(0.03)\end{array}$ \\
\hline $\begin{array}{l}\text { C List } \\
\text { incumbent }^{c}\end{array}$ & $\begin{array}{c}11.47 * * * \\
(2.35)\end{array}$ & $\begin{array}{c}11.44 * * * \\
(2.34)\end{array}$ & $\begin{array}{c}11.48 * * * \\
(2.37)\end{array}$ & $\begin{array}{l}11.46^{* * * *} \\
(2.34)\end{array}$ & $\begin{array}{c}-12.2 * * * \\
(2.78)\end{array}$ & $\begin{array}{c}11.48 * * * \\
(2.34)\end{array}$ & $\begin{array}{c}10.42 * * * \\
(1.96)\end{array}$ \\
\hline $\begin{array}{l}\text { C SMD } \\
\text { incumbent }^{\mathrm{c}}\end{array}$ & $\begin{array}{c}5.00 * * * \\
(1.30)\end{array}$ & $\begin{array}{c}4.99 * * * \\
(1.31)\end{array}$ & $\begin{array}{c}4.97 * * * \\
(1.29)\end{array}$ & $\begin{array}{c}5.06 * * * \\
(1.32)\end{array}$ & $\begin{array}{c}-6.50 * * * \\
(1.46)\end{array}$ & $\begin{array}{c}5.09 * * * \\
(1.30)\end{array}$ & $\begin{array}{c}0.33 \\
(3.37)\end{array}$ \\
\hline Constant & $\begin{array}{c}-10.7 * * * \\
(1.20)\end{array}$ & $\begin{array}{c}-10.6^{* * *} \\
(1.25) \\
\end{array}$ & $\begin{array}{c}-10.5 * * * \\
(1.24)\end{array}$ & $\begin{array}{c}-10.0 * * * \\
(1.70)\end{array}$ & $\begin{array}{c}17.7 * * * \\
(1.22)\end{array}$ & $\begin{array}{c}-10.6^{* * *} \\
(1.20)\end{array}$ & $\begin{array}{c}-12.6 * * * \\
(1.84)\end{array}$ \\
\hline Sigma & $\begin{array}{l}7.41^{* * *} \\
(1.05)\end{array}$ & $\begin{array}{c}7.42 * * * \\
(1.06)\end{array}$ & $\begin{array}{c}7.44 * * * \\
(1.06)\end{array}$ & $\begin{array}{c}7.41 * * * \\
(1.05)\end{array}$ & $\begin{array}{l}- \\
-\end{array}$ & $\begin{array}{l}7.41 * * * \\
(1.06)\end{array}$ & $\begin{array}{c}7.41 * * * \\
(0.91)\end{array}$ \\
\hline $\mathrm{N}$ & 1121 & 1121 & 1121 & 1121 & 1121 & 1121 & 630 \\
\hline Censored N & 141 & 141 & 141 & 141 & - & 141 & 57 \\
\hline Log-likelihood & -3501.86 & -3502.61 & -3505.02 & -3501.08 & -4047.81 & -3501.19 & -2027.49 \\
\hline $\begin{array}{l}\text { Nagelkerke } \mathrm{R}^{2} / \\
\text { adj. } \mathrm{R}^{2}\end{array}$ & 0.40 & 0.40 & 0.40 & 0.40 & 0.55 & 0.40 & 0.35 \\
\hline
\end{tabular}


Existing literature suggests that in Germany and other Western democracies with significant immigration, right-wing parties contribute to migrants' descriptive representation less often than left-wing parties (e.g. Bloemraad, 2013: 664). Accordingly, we find that double candidates of immigrant-origin are a priori less likely to run for right-leaning parties:

- $\quad \mathrm{CSU} / \mathrm{CDU} \mathrm{n}=7$

- $\quad$ FDP $n=8$

- $\quad$ Grüne $n=21$

- $\quad$ Linke $n=18$

- $\quad$ SPD $n=19$.

Figure A3 shows also that the distribution of the foreigner share is strongly left-skewed in the group of centre-right CIOs (CDU/CSU and FDP), while the picture is more balanced for left-wing CIOs. In other words, almost all CIOs running in a SMD with a high foreigner share are left-wing. This suggests that our results are driven by left-wing parties. However, these patterns do not thwart the overall relationship because immigrant-origin candidates are a lot less likely to run for centre-right parties and their distribution is strongly biased toward smaller foreigner shares. Model 7 in table A3 further supports this view by replicating the main model for left-wing parties only. As can be seen, the results are very similar. 
Figure A3. Scatterplots of relative list positions and local foreigner shares by party
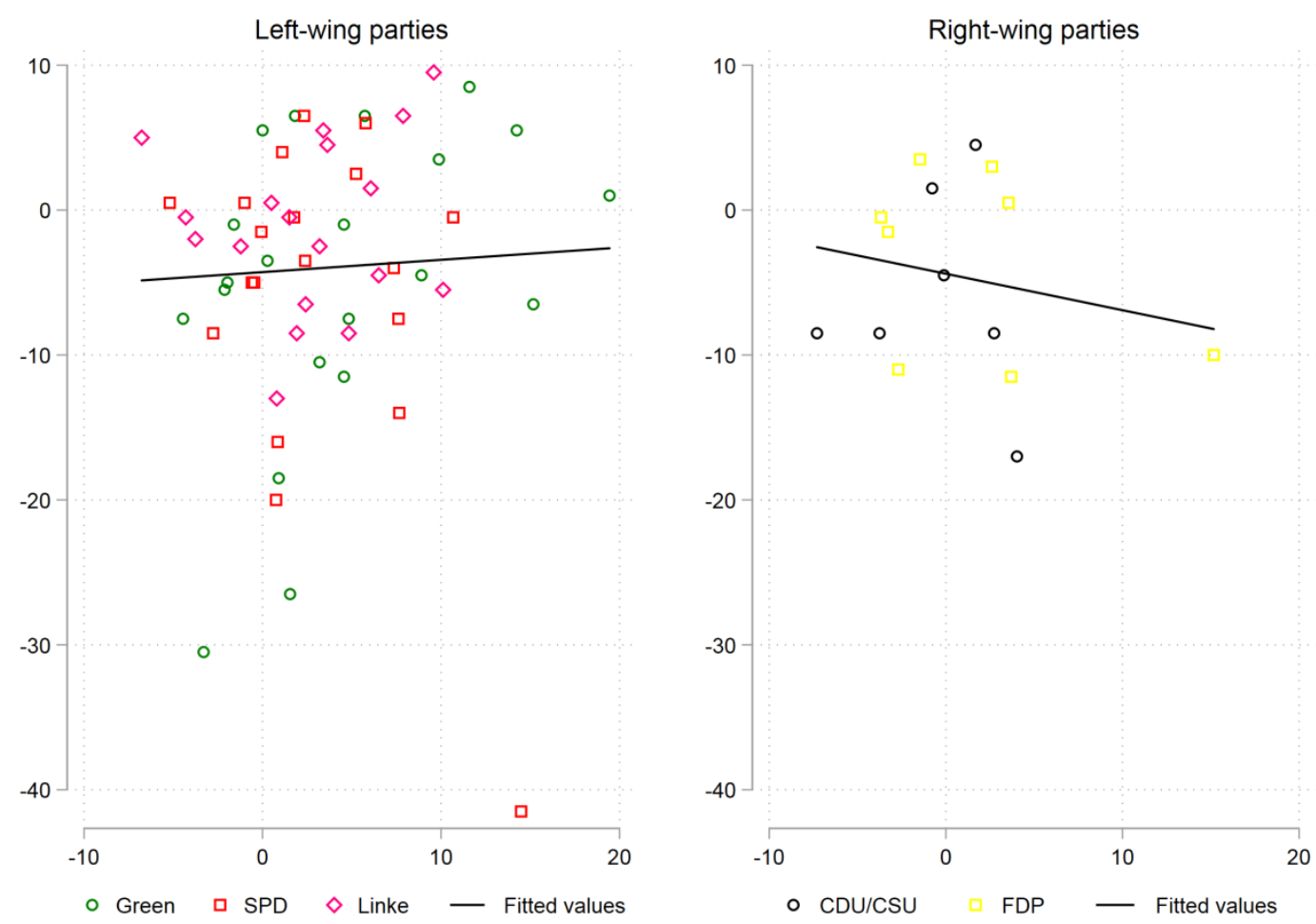

Note: own calculation based on data described in text 


\section{Aufsatz 3}

Lucas Geese \& Carsten Schwemmer (2019) MPs' principals and the substantive representation of disadvantaged immigrant groups, West European Politics, 42:4, 681-704, https://doi.org/10.1080/01402382.2018.1560196

Das folgende Manuskript ist der Post-print des Aufsatzes, der von der Redaktion der Fachzeitschrift West European Politics (ISSN: 0140-2382) zur Publikation angenommen wurde. 


\section{MPs' Principals and the Substantive Representation of Disadvantaged Immigrant Groups}

\section{Lucas Geese $^{\mathrm{a}}$ and Carsten Schwemmer ${ }^{\mathrm{b}}$}

${ }^{a}$ Faculty for Social Sciences, Economics, and Business Administration, University of Bamberg, Bamberg, Germany, Lucas.Geese@uni-bamberg.de, phone: +49(0)951-863-3010 (Corresponding author); ${ }^{b}$ Faculty for Social Sciences, Economics, and Business Administration, University of Bamberg, Bamberg, Germany, Carsten.Schwemmer@uni-bamberg.de, phone: +49(0)951-863-2736

Lucas Geese is a lecturer in the Faculty for Social Sciences, Economics, and Business Administration at the University of Bamberg, Germany. His research interests focus on political institutions, minority representation, parliaments and methods of textual analysis.

Carsten Schwemmer is a lecturer at the University of Bamberg. He is working on computational methods for conducting social research with a special focus on natural language processing. He is especially interested in political sociology, ethnic minority studies and social media.

\section{Funding}

This work was supported by the German Research Foundation (DFG) under Grant SA 2160/31 (principal investigator: Thomas Saalfeld)

\section{Acknowledgments}

Earlier versions of this article were presented at the ABC Conference 2016 in Bamberg and at the 'Anxieties of Democracy' workshop 2017 in Mainz. We thank Thomas Saalfeld, Marc Helbling, Jorge M. Fernandes, Henning Bergmann, Javier Martínez Cantó, Simon Fink, Daniel Gillion, Margret Hornsteiner, Stefanie John, Ira Katznelson, Caroline Schultz and two anonymous reviewers for helpful comments and suggestions. We also thank Magdalena Stiegler, Elena Maier, David Beck, Johannes Geiger and Emanuel Slany for research assistance, and Joanna MacLeod for proofreading. Data have been obtained within the project 'Pathways to Power: The Political Representation of Citizens of Immigrant Origin in Seven European Democracies (PATHWAYS)'. This project was funded by the ANR (France), DFG (Germany), ESRC (United Kingdom) and NWO (Netherlands) under the Open Research Area (ORA+) framework. The PATHWAYS consortium is formed by the University of Amsterdam (Professor Jean Tillie), the University of Bamberg (Professor Thomas Saalfeld), the University of Leicester (Professor LauraMorales) and the CEVIPOF-Sciences Po Paris (Professor Manlio Cinalli)

\section{Abstract}


This article provides an alternative understanding of the substantive representation of immigrant-origin citizens compared to previous work in the 'politics of presence' tradition. Rather than assuming that the representational activities of members of parliaments (MPs) are underpinned by intrinsic motivations, it highlights extrinsic motives. Drawing on principalagent theory, the article conceptualises MPs as delegates who are to act on behalf of their main principals, constituents and party bodies. This approach permits the rigorous analysis of the impact of electoral rules, candidate selection methods and legislative organisation on substantive representation. Based on an analysis of more than 20,000 written parliamentary questions tabled in the 17th German Bundestag (2009-13), empirical findings suggest that electoral rules do not influence the relationship between MPs and their principals in relation to the substantive representation of disadvantaged immigrant groups, however, results indicate that candidate selection methods as well as powerful parliamentary party group leaderships do.

\section{Keywords}

Substantive representation; Immigrant-origin citizens; Parliamentary questions; Electoral rules, Candidate selection, Legislative organisation 


\section{Introduction}

The normative ideal of democratic representation (e.g. Dahl 1971) suggests that as more immigrant-origin residents acquire citizenship and thus the right to vote, their interests should find more consideration in the parliamentary activities of members of parliament (MPs). Indeed, the relevance of this ideal should not be underestimated, given that immigrants and their descendants remain socially and economically disadvantaged in most Western democracies of immigration (Alba and Foner 2015). Consequently, political scientists are called for to examine the mechanisms underlying the substantive representation of disadvantaged immigrant groups.

Conceptually, substantive representation refers to whether MPs 'act in the interest of' citizens, while descriptive representation refers to whether MPs' sociodemographic features 'stand for' a certain group of citizens (Pitkin 1967). Despite this conceptual differentiation, however, normative claims of a connection between the two concepts of representation (Mansbridge 1999; Phillips 1995) has inspired the lion's share of previous research on immigrants' substantive representation (e.g. Aydemir and Vliegenthart 2016; Saalfeld 2011; Saalfeld and Bischof 2013; Wüst 2014a). Doubtlessly, this literature has advanced our understanding considerably, confirming by and large a link between the descriptive and substantive representation of immigrant-origin citizens. Nevertheless, it is no secret that immigrant-origin citizens remain descriptively underrepresented in Western European parliaments (Alba and Foner 2015; Bird et al. 2011; Bloemraad and Schönwälder 2013). Therefore, this group of citizens has to rely to a considerable extent on the level of substantive representation provided by native MPs. There is, however, a dearth of research on immigrants' substantive representation unrelated to MPs' own national or ethnic backgrounds. 
Consequently, rather than relying on the assumption of intrinsically motivated 'descriptive' representatives, we think in this paper of MPs as agents in a principal-agent relationship, with local voters as well as political party bodies inside and outside parliament being the most important principals (Carey 2009; Mitchell 2000; Müller 2000). Speaking on behalf of immigrants and their descendants is understood as being part of MPs' strategies to please the demands of their principals. Yet, the incentive to please the demands of one principal at the expense of another one is a function of the institutional environment. On one hand, MPs favour the demands of a centralised party body under party-centred electoral rules, a centralised candidate selection method and due to powerful parliamentary party groups (PPGs). On the other, they are 'pulled' towards local demands by candidate-centred electoral rules and a localised candidate selection method (Carey and Shugart 1995; Gallagher 1988; Strøm 1997).

The question arises what happens when the incentives encoded in these institutional features conflict (Martin 2014; Preece 2014). Do MPs remain responsive to the local demands of immigrant-origin citizens when a decentralised candidate selection method clashes with partycentred electoral rules? Do they remain responsive to the demands of the PPG leadership when electoral rules are candidate-centred? To examine these questions, we turn to a case study of MPs' legislative behaviour in the German Bundestag, a complex institutional context combining mixed electoral rules with a localised candidate selection method and tightly organised PPGs. Here, MPs are 'pulled' by their principals' demands in different directions, thus providing researchers the opportunity to better disentangle the effects of institutional variables while holding country-specific context fixed. Empirically, this study is based on a dataset of all MPs serving in the $17^{\text {th }}$ Bundestag (2009-13), combined with a semi-automated content analysis of more than 20,000 of their parliamentary questions (PQs) for written answer. 
Quantitative analyses of this dataset suggest that principals' demands are important determinants of the substantive representation of disadvantaged immigrant groups in MPs' PQs. However, our findings provide little support that different electoral rules moderate MPs' attentiveness towards the demands of their principals. A localised candidate selection on the one hand and powerful PPG leaderships on the other, by contrast, are found to be more consequential for the substantive representation of disadvantaged immigrant groups.

\section{Institutional Context and the Substantive Representation of Disadvantaged Immigrant Groups}

A major controversy in political science is the question of whether MPs should be conceptualised as trustees, who act based on their own conscience, or as delegates, who act based on the instructions of others (Converse and Pierce 1986; Pitkin 1967). Conceptualising MPs as trustees means in large parts to assume that MPs' intrinsic motivations underlie their legislative behaviour. This is basically what normative arguments in the 'politics of presence' school of thought are based on. In order to represent the interests of disadvantaged groups, representatives need to have a thorough understanding of and similar life experiences to the represented, which can be best achieved by descriptive representation (Mansbridge 1999; Phillips 1995: 159). Previous empirical research in this line of thought (e.g. Aydemir and Vliegenthart 2016; Saalfeld 2011; Saalfeld and Bischof 2013; Wüst 2014a) is thus widely based on the assumption that MPs' legislative behaviour hinges on their intrinsic motivations, that is, on the trustee notion of substantive representation.

Conceptualising MPs as delegates, however, makes us aware that substantive representation may also be based on demands external to MPs' conscience and personal experiences. In this 
view, MPs act as agents of principals who control access to certain goods that MPs value (Carey 2009; Mitchell 2000; Müller 2000). The assumption is that MPs are driven by their ambition to reach certain career-related goals, ordered in the following way. First of all, MPs need to achieve reselection as a necessary precondition for their second goal, reelection, which in turn is a necessary condition for the achievement of their third goal, access to positions of influence within parliament, such as committee membership and chairs or frontbench membership (Strøm 1997). The achievement of the first goal, reselection, is in most parliamentary democracies controlled by parties' nomination conventions (Müller 2000). The second goal, reelection, can only be achieved, if enough voters support the candidate or the party list bearing him/her (Mitchell 2000). The third goal, positions of legislative influence, is in most cases under control of the leadership of the PPG (Carey 2009). Thus, MPs typically find themselves in the difficult situation of having to please the demands of (at times) three different principals: voters, party selectorates and PPG leaders.

In the view of principal-agent theory, MPs' acting on behalf of disadvantaged immigrant groups can be therefore understood as being part of a strategy supposed to please the demands of one or several principals. The extent to which the demands of one principal outweigh the demands of another one, however, depends on the relative value of the resources controlled by each principal, which is determined by the rules of the game, that is, their institutional environment (cf. Carey 2009: 14). Among the most important institutional variables are electoral rules, candidate selection methods and the internal organisation of parliaments.

Electoral rules, to begin with, are commonly thought to determine the relative weight of local voter groups for MPs' reelection prospects relative to the weight of the party branch controlling the candidate selection process. Under closed-list PR elections, voters have little leverage to change the electoral fate of individual candidates, given they are confronted with fixed and often long lists of candidates, which voters can only take or defect as a whole 
(Carey and Shugart 1995; Mitchell 2000; Shugart et al. 2005). The list position allocated in the selection process will thus determine MPs' future electoral prospects, such that MPs should have strong incentives to follow the demands of a party selectorate (Carey 2009). By contrast, in more candidate-centred systems, like single-member district elections, voters have more influence over the electoral fate of individual candidates, such that MPs should cultivate a relatively stronger local voter support (Carey and Shugart 1995; Mitchell 2000). Therefore, MPs should see more reasons to provide substantive representation in response to local concentrations of immigrant-origin citizens when elected in single-member districts. Conversely, the demands of national party bodies should weigh stronger on MPs' shoulders with regard to the representation of immigrants' interests under closed-list PR rules.

The candidate selection method is another factor that may affect the relationship between MPs and their principals. As already mentioned, reselection is a necessary precondition for all other career-related goals, such that MPs can be assumed to owe part of their loyalty to the gatekeepers in the candidate-selection process (Müller 2000). In this respect, the degree of territorial decentralisation is an important dimension of candidate selection (Rahat and Hazan 2001). Arguably, local party organisations should attach greater weight to the local visibility of their parliamentary representatives while national party headquarters should value MPs' efforts to cultivate a national party reputation (Gallagher 1988: 15; Karlsen and Narud 2013). Given the reputation and visibility of national MPs, their legislative behaviour should serve local party branches as an important campaigning tool for the purpose of tapping into local voter markets of immigrant-origin citizens in municipality elections. If local party branches have leverage over the reselection of MPs, they possess a means to that end, that is, the means to make their parliamentary agents speak on behalf of disadvantaged immigrant groups. Thus, the link between local concentrations of immigrant-origin citizens and their substantive representation may be the result of a localised candidate selection method. On the other hand, 
if the national party headquarters maintain control over the reselection of MPs, the demand of this principal should determine immigrants' substantive representation more strongly.

Legislative organisation is a third institutional feature that is particularly consequential for the principal-agent relationship between PPG leaderships and individual MPs. Strøm (1998), distinguishes a vertical and a horizontal dimension of legislative organisation. Vertically, the building blocks of parliaments are hierarchically organised PPGs (Saalfeld and Strøm 2014). At the top of this hierarchy, PPG leaderships seek to further the collective goals of the national party in terms of policy, offices and votes (Strøm and Müller 1999). To achieve these goals, however, PPG leaders depend on the collective effort of the entire party group (Müller 2000), although individual MPs sometimes face deviating cross-pressure from competing principals (Carey 2009). In order to incentivise MPs to work towards the collective goals of the party despite competing demands, PPG leaders often have a number of disciplinary instruments at their disposal: patronage and control of MPs' promotion to influential legislative or execute office, assignment to or withdrawal from certain committees, access to the parliamentary floor/ rapporteurship, access to the media, and benefits such as business trips, office space, staff and a variety of other perks (Bailer 2017; Bowler et al. 1999; Carey 2009; Sieberer 2006; Strøm 1997). Some of these resources can strongly affect MPs' individual vote-seeking and policy goals. For example, appointment to a leadership position in the PPG can enhance MPs' policy influence, while access to the parliamentary floor in a well-publicised debate provides a public platform to enhance the MP's status among constituents or the local party base.

Disciplinary measures are commonly considered important instruments for the purpose of accomplishing party unity when bills are voted on in the plenary, thus ensuring the collective decision-making ability of the parliament (Bailer 2017; Bowler et al. 1999; Sieberer 2006). However, focusing solely on legislative voting in the plenary would neglect the horizontal 
dimension of legislative organisation, that is, the role of specialised committees. Committees play a crucial role in most parliaments as they constitute the arena in which bills are considered and amended before being mainly 'waved through' in the plenary (Cox and McCubbins 2007: 9-12). Indeed, the scarcity of time and the fact that law-making necessitates a sophisticated level of policy-specific expertise on the part of MPs makes committee specialisation a necessary and important feature of parliamentary politics (Strøm 1998: 24-27).

Therefore, by necessity, PPG leaders have to consider that policy-making takes place in various policy jurisdictions. Plausibly, the need for an efficient division of labour is intimately connected with the principal-agent relationship between PPG leaderships and their MPs. In that sense, committees can be understood as an extension of legislative party power (Cox and McCubbins 2007; Miller and Stecker 2008; Strøm 1998). On the one hand, the assignment of MPs to the various specialised committees ensures an efficient division of labour within the PPG. On the other, the tight vertical organisation within PPGs provides PPG leaders with a vertical grip over their MPs that often effectively reaches down into MPs' committee-based work. If the PPG leadership possesses effective monitoring devices and has at its disposal the sort of disciplinary measures already discussed, it possesses effective means of incentivising individual MPs to further the collective goals of the party within the confines of the policy jurisdictions of the MP's committee specialisation (Damgaard 1995). Based on these considerations, it is thus plausible to assume that the extent to which MPs' committee assignments shape their legislative behaviour reflects the extent to which they serve their PPG leaderships as policy-specialised agents. Therefore, MPs should have incentives to further the interests of disadvantaged immigrant groups if this is a policy goal of their PPG leaderships in the policy jurisdiction of their committees. 


\section{Parliamentary Questions and the Substantive Representation of}

\section{Disadvantaged Immigrant Groups in the German Bundestag}

To examine this theoretical framework, we focus our study on Germany for two main reasons. First, Germany is a very relevant case to the study of immigrants' substantive representation. Germany accounts for $20 \%$ of the entire immigrant population in the European Union (OECD and EU 2015: 40) and the immigrant-origin electorate is sizeable, amounting to $9 \%$ in the 2013 Bundestag elections (Bundeswahlleiter 2013). At the same time, however, there are strong structural inequalities separating immigrants' social and economic situations from those of the German majority population (cf. Die Beauftragte der Bundesregierung für Migration, Flüchtlinge und Integration 2016).

Second, Germany's institutional environment offers the opportunity to analyse and contrast the effects of institutional variables on the relationship between MPs and their principals. German MPs find themselves in a complex institutional environment combining mixed electoral rules with a localised candidate selection procedure and tightly organised PPGs. This environment provides researchers the opportunity to better disentangle the effects of these factors while holding constant influences of country-specific context (e.g. Moser and Scheiner 2012: 46). Indeed, it remains a matter of controversy whether electoral rules trump the effects of candidate selection methods and legislative organisation, or vice versa. Shugart and coauthors (2005: 441) argue, for example, that parties and MPs alike respond mainly to voters' informational demands encoded in the electoral system, and not, for example, to partyrelated candidate selection procedures. However, others have argued that centralised candidate selection methods and powerful PPG leaders weaken MPs constituency relations despite strong personal vote-seeking incentives encoded in electoral rules (Martin 2014; 
Preece 2014). In this article, we take these opposing views as empirical questions, leveraging Germany as an institutional environment in which principals 'pull' their MPs into different directions.

To pursue these empirical questions, we follow previous research and draw on parliamentary questions (PQs) for written answer (Schriftliche Fragen) as indicators of substantive representation (Aydemir and Vliegenthart 2016; Saalfeld 2011; Saalfeld and Bischof 2013; Wüst 2014a). PQs are well suited for the purpose of dealing with our research question, because they indicate MPs' personal efforts to represent the interests of disadvantaged immigrant groups in response to external demands. Other legislative activities, for example speeches or roll call votes, are strictly controlled by the PPG leadership, especially in a strongly party-controlled parliament such as the Bundestag (Depauw and Martin 2009; Proksch and Slapin 2015). In comparison, MPs can use PQs relatively freely to raise the attention of the government to certain issues, to acquire information from the bureaucracy or to claim credit for their PQs in their websites, social media or local newspapers (Martin 2011b; Rozenberg and Martin 2011; Russo and Wiberg 2010).

The first major question we seek to answer is whether MPs' election in local constituencies or whether their selection as local candidates determines their responsiveness to local concentrations of immigrant voters. In Germany's electoral system, 299 MPs are elected in single-member plurality districts (SMD tier), and a slightly larger number of MPs is elected in 16 multi-member districts under rules of closed-list proportional representation (PR tier). The system is compensatory in that parties' vote shares in the PR tier determine their overall seat shares, that is, seats won in the SMD tier are used first to fill the allocated seat shares and thereafter remaining seats are drawn from state-based party lists. As MPs are elected under different electoral rules in the same system, it is often assumed that mixed-member systems generate a 'mandate-divide' between the two types of MPs, that is, SMD MPs' representative 
behaviour focuses more strongly on local constituents, while PR MPs focus mainly on national party bodies (for an excellent literature review see Manow 2013). Scholars of mixedmember systems, however, have expressed scepticism regarding the mandate-divide thesis, arguing that behavioural differences between the two types of MPs blur due to other institutional influences affecting MPs' behaviour in similar ways across electoral tiers (e.g. Crisp 2007; Ferrara et al. 2005; Manow 2013).

In the German case, such arguments often highlight how candidates are selected to run for the Bundestag (Manow 2013). Formally, the electoral law stipulates that candidates in the SMD tier must be selected in local constituencies while candidates in the PR tier must be selected at nomination conferences at the upper regional level (Detterbeck 2016). However, the electoral law permits candidates to run as 'dual candidates', that is, in both electoral tiers simultaneously ${ }^{1}$. In fact, dual candidacy is common, because voters reward parties electorally for the local presence of candidates (Ferrara et al. 2005; Hainmueller and Kern 2008). Therefore, parties have vote-seeking incentives to require that their candidates are selected in the SMD tier before being allowed access to promising party list positions in the PR tier (Detterbeck 2016; Manow 2013: 289). In other words, as local re-selection is a precondition for realistic list positions in the PR tier, local reselection is de-facto a requirement for MPs' reelection in both electoral tiers. Therefore, SMD and PR MPs alike may have incentives to represent local constituencies in their PQs.

In line with Crisp, we argue that the finding of behavioural homogeneity across electoral tiers can be taken as evidence that the incentives institutionalized in the electoral tiers are being trumped by the candidate selection process (Crisp 2007: 1462). In other words, if the locus of candidate selection were the driving force behind German MPs' responsiveness to the demands of local immigrant-origin citizens and not their election in single-member districts, 
then MPs should respond to the share of immigrant-origin citizens in the constituency where they were locally selected. Thus, our first hypothesis reads:

MPs are more responsive to immigrant-origin citizens' interests the more immigrant-origin voters reside in their local constituencies, regardless of their election mode (H1).

The second major question is whether MPs' responsiveness to the demands of their PPG leaderships to speak on behalf of disadvantaged immigrant groups is mainly influenced by electoral rules or by the internal organisation of the parliament. In terms of legislative organisation, the Bundestag could be described as a party-controlled Arbeitsparlament ('working parliament') based on the division of labour in policy-specialised committees mirroring the government structure (Ismayr 2012: 162; Miller and Stecker 2008). PPG leaderships maintain strong control over their MPs' committee work, as they have the prerogative of assigning MPs to, and withdrawing them from committees (Damgaard 1995; Miller and Stecker 2008). The strong role of parties is also reflected in the fact that committees work behind closed doors, therefore only visible to the PPG leadership as a principal. Party control is further ensured by weekly meetings of the PPGs' working groups, which mirror the committee structure and prepare the parties' positions in the committee (Miller and Stecker 2008). If MPs refuse to work in line with the policy goals of the party, the PPG leadership can apply several sanctions. These range from subtle pressure, to the dissenting MP's withdrawal from the committee, or the ultimate denial of promotion within the hierarchy of the PPG (Damgaard 1995; Ismayr 1992: 169).

While it remains relatively undisputed that PQs can serve MPs for the purpose of cultivating local voter support (Fernandes et al. 2018; Martin 2011a; Russo 2011; Saalfeld 2011), it is not as common to use PQs as a measure of MPs' responsiveness to the demands of PPGs (but see Bailer 2011). After all, PQs are widely considered a legislative instrument free of party control. Nevertheless, we argue that PQs matter to the principal-agent relationship between 
PPG leaderships and MPs, albeit in an indirect way. Our argument is based on the intuition that MPs serve their PPG leaderships as policy experts in specialised committees, as outlined in the previous section of this paper. PQs are informative for this principal-agent relationship, because they afford MPs a low-cost opportunity to gather relevant information from government departments to support their daily committee-based work (Bailer 2011; Russo and Wiberg 2010). To comply with their role as policy-specialised agents, MPs may thus ask PQs on issues in their area of expertise. Consequently, a close relationship between MPs' committee memberships and the type of PQs they ask should reflect their responsiveness to the expectations of their PPG leaders to further collective party goals within the policy jurisdictions of their committees. Since certain committees are more likely to deal with matters of immigrants' disadvantage, for example the committee for social affairs or education rather than the committee for environment or defence, MPs sitting on these committees should be more likely to ask PQs related to immigrant matters. We thus expect that:

MPs are more responsive to immigrant-origin citizens' interests when they sit on migrantrelated committees $(\mathrm{H} 2 \mathrm{a})$.

However, the extent to which the improvement of the living conditions of disadvantaged immigrant groups is defined as a policy goal should vary across PPGs. Plausibly, this variation is reflected in parties' election manifestos, guiding MPs in their pursuit to please the demands of their PPG leadership. For this reason, we expect an interaction between MPs' policy specialisation, reflected in their committee memberships, and the extent to which the integration of immigrants is reflected as a policy goal in the party manifesto (herein called the integration-relatedness of party manifestos).

We thus hypothesise that 
the committee effect described in H2a depends on the integration-relatedness of the party manifesto (H2b).

Finally, the question remains to what extent the effects of legislative organisation are countervailed by electoral rules. As Carey (2009: 133) explains 'virtually all legislators are subject to influence by at least one principal - their legislative party leadership', but 'legislators' electoral connection to voters might pull them in directions contrary to the demands of legislative party leaders'. Accordingly, we might expect that the influence of PPG leaders to ask PQs on behalf of disadvantaged immigrant groups measured by an interaction of committee membership and integration-related party ideology will be weaker for MPs elected in SMDs as compared to MPs elected in the PR tier. Conversely, if legislative organisation can trump effects of electoral rules entirely, we would expect that

the interaction effect of committee membership and integration-related party ideology described in $\mathrm{H} 2 \mathrm{~b}$ works regardless of electoral rules (H2c).

\section{Data and Methods}

\section{Measuring Substantive Representation in Parliamentary Questions}

To test the hypotheses laid out in the previous section, we compiled all 20,130 PQs tabled by individual MPs in the $17^{\text {th }}$ German Bundestag. In order to identify PQs tabled on behalf of disadvantaged immigrant groups, we focus on the representation of their objective interests rather than on the representation of their subjective interests (for a detailed discussion see 
Swain 1993: 6). That is, PQs are understood to be substantively representative if they raise attention to immigrants' unequal living conditions, for example in terms of level of income, physical well-being or employment status, and/or demand the integration of immigrant-origin residents into German society. Integration refers here, according to Alba and Foner (2015: 5), to processes that increase the opportunities of immigrants and their descendants in major institutions such as the educational and political system and the labour and housing market. In order to identify PQs tabled on behalf of disadvantaged immigrant groups, herein called integration-related $P Q s$, we combine human and dictionary-based machine coding. A detailed description of the text coding procedure and its validation, the final list of key words, as well as two examples of such questions are provided in the appendices A1 and A2 to this paper. Based on this coding, the final measure of our dependent variables is the count of integrationrelated PQs per MP.

\section{Independent and Control Variables}

We measure the magnitude of the local demand of immigrant-origin citizens as the share of foreign nationals in the local district ${ }^{2}$ and connect this information to all MPs who were running in the election as SMD tier candidates. Thus, all dually nominated MPs are linked to the constituencies in which they were selected to run as SMD tier candidates. PR tier legislators who did not run as a candidate in a district race $(2.3 \%$ of all legislators $)$ were excluded from the analysis. Of course, using the percentage of foreign nationals as a proxy for the immigrant-origin electorate at the constituency-level is not ideal. Nevertheless, it is the only immigrant-related indicator available at the constituency-level, and given it is highly correlated $(\mathrm{r}=0.78)$ with the group of naturalised residents of immigrant-origin at the level of differently drawn administrative districts (Wüst 2014b) we take this indicator as a reasonable 
approximation of the immigrant-origin electorate. The difference between SMD and PR tier MPs is captured in a dummy variable which takes values of one for SMD MPS.

To code the integration-relatedness of party manifestos, we utilise data from the Comparative Manifesto Project for the 2009 Bundestag election, following previous work in the field (Alonso and Fonseca 2012; Volkens et al. 2015; Wüst 2016). For a detailed description of the coding, please see the online appendix A3. Higher values on this continuous scale indicate more integration-relatedness. While the two right-wing parties (CDU/CSU and FDP) score low on this scale (7.121 and 6.935), the three left-wing parties (SPD, Greens and The Left) score considerably higher $(16.894,16.435$ and 24.91). Additionally, party differences are captured in a dummy for the simple left/right distinction. For the purpose of identifying migrant-related committees, we rely on a modified categorisation of the dichotomous categorisation scheme proposed by Wüst $(2011)^{3}$.

In order to test whether the theoretical framework proposed in this article contributes significantly to established explanations, we also add a control variable for the effect of descriptive representation as the main focus of previous research. We identified all MPs as being of immigrant-origin $(n=24)$ if they were born with a foreign nationality or if one of the respective person's parents was born with a foreign nationality. In addition to that, we control for the total number of $P Q s$ asked per MP. Since the extent to which PQs are used overall should depend on MPs' government or opposition status as well as on their seniority and career stages (Bailer and Ohmura 2018), we control for these factors implicitly when including this variable. Table 1 provides a descriptive overview of all variables. 


\begin{tabular}{lcccc}
\hline & Min & Max & $\begin{array}{c}\text { Mean } / \\
\text { Share }\end{array}$ & SD \\
\hline $\begin{array}{l}\text { No. of integration-related PQs } \\
\text { \% Foreign Nationals in District }\end{array}$ & 0 & 52 & 0.83 & 3.86 \\
PR (0) vs. SMD tier(1) & 0 & $28 \%$ & $9 \%$ & $5 \%$ \\
Party: Right (0) vs. Left (1) & 0 & 1 & 0.47 & - \\
& & & 0.46 & - \\
$\begin{array}{l}\text { Integration-relatedness of party } \\
\text { manifesto }\end{array}$ & 6.94 & 24.91 & 12.42 & 6.25 \\
$\begin{array}{l}\text { Other (0) vs. immigrant-related } \\
\text { committee (1) }\end{array}$ & 0 & 1 & 0.47 & - \\
$\begin{array}{l}\text { Native (0) vs. migratory } \\
\text { background (1) }\end{array}$ & 0 & 1 & 0.04 & - \\
Total no. of PQs & 0 & 196 & 30.68 & 44.58 \\
\hline Observations & 637 & & & \\
\hline
\end{tabular}

\section{Statistical Model}

The empirical modelling strategy must take into account two related methodological aspects. First, as our unit of analysis is the MP and the dependent variable captures counts of integration-related questions asked per MP, negative-binomial regression models are an appropriate choice. ${ }^{4}$ Second, the share of zeros in our dependent variable amounts to $82.7 \%$. Zeros may be generated according to two different mechanisms. First, an MP decides not to ask a single integration-related PQ. Second, an MP decides not to ask any PQs at all. The latter mechanism is strongly related to the tendency of MPs representing government parties to ask no or only few PQs, while MPs of opposition parties typically ask a lot more PQs. Obviously, a major precondition to the tabling of integration-related questions is that an MP asks PQs at all. In our dataset 399 out of 637 MPs asked at least one PQ, and 110 MPs asked at least one integration-related question. 


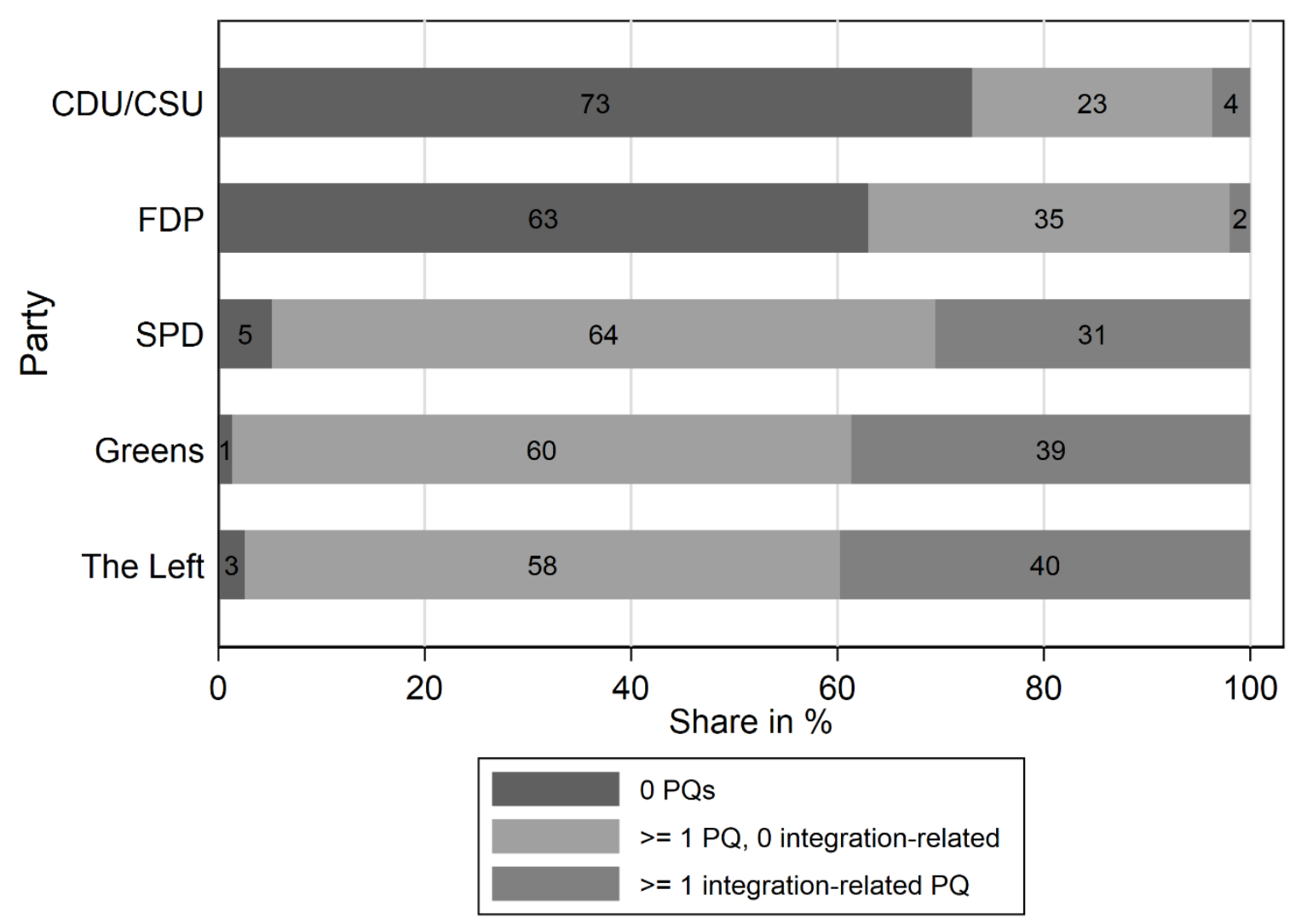

Figure 1. Percentages of MPs by party tabling no $P Q$, at least one $P Q$ but no integration-related PQs, or at least one integration-related $P Q$.

Figure $1^{5}$ confirms this suspicion by showing the distribution of MPs who either tabled no PQs, at least one PQ but no integration-related PQs, or at least one integration-related PQ. In comparison to government MPs (CDU/CSU, FDP), members of opposition parties (The Left, SPD, Greens) are more likely to table more PQs overall. This is especially problematic since in the legislative term under study the division between opposition and government is clearcut with regard to the left/ right divide. In order to better disentangle party and opposition effects and better handle the zero-inflation in our dependant variable we fit zero-inflated negative binomial regression models. These models are mixture models that combine two regression equations: a logit model to explain the zero inflation, and a negative binomial model to explain non-zero counts in the dependant variable (Cameron and Trivedi 2013: 11176). In order to explain MPs' propensity of asking at least one integration-related PQ, we include the total number of PQs asked by each MP in the inflation equation ${ }^{6}$. Doing so allows 
us to control for factors that make MPs ask few or no PQs at all, as outlined in the previous section, in the explanations of zero-observations.

\section{Results}

In Table 2 we present the results of four estimated zero-inflated regression models. The models estimate the effects of the independent and control variables on the number of integration-related PQs in the count regression equation. Model 1 includes all independent variables without interactions, indicating that MPs ask more integration-related PQs the more immigrants reside in their constituencies, the higher the integration-relatedness of their parties' manifestos and if they sit on migration-related committees.

Model 2 extends the regression model by the interaction between the local share of foreign nationals and the distinction between MPs' election modes to test whether the constituency effect works regardless of the electoral tier (H1). Here, the coefficient for the share of foreign nationals, which stands for the constituency effect of list MPs, is positive and statistically significant at $\mathrm{p}<0.1$. By contrast, neither the coefficient of the SMD tier, which stands for the average difference between list and SMD MPs, nor the coefficient of the interaction term, which stands for the difference of the constituency effect for SMD MPs relative to list MPs, reaches conventional levels of statistical significance. 
Table 2: Determinants of the number of integration-related $\mathrm{PQs}$

\begin{tabular}{|c|c|c|c|c|}
\hline & $\begin{array}{c}\text { Model } 1 \\
\text { b/se }\end{array}$ & $\begin{array}{c}\text { Model } 2 \\
\mathrm{~b} / \mathrm{se}\end{array}$ & $\begin{array}{c}\text { Model } 3 \\
\text { b/se }\end{array}$ & $\begin{array}{c}\text { Model } 4 \\
\text { b/se }\end{array}$ \\
\hline \multicolumn{5}{|l|}{$\begin{array}{l}\text { Negative binomial count } \\
\text { model: }\end{array}$} \\
\hline$\%$ Foreign Nationals ${ }^{\mathrm{a}}$ & $\begin{array}{l}0.06^{* *} \\
(-0.03)\end{array}$ & $\begin{array}{c}0.06^{*} \\
(-0.03)\end{array}$ & $\begin{array}{l}0.06^{* *} \\
(-0.02)\end{array}$ & $\begin{array}{l}0.06^{* *} \\
(-0.03)\end{array}$ \\
\hline SMD MP & $\begin{array}{c}-0.14 \\
(-0.25)\end{array}$ & $\begin{array}{l}-0.15 \\
(-0.25)\end{array}$ & $\begin{array}{c}-0.13 \\
(-0.25)\end{array}$ & \\
\hline $\begin{array}{l}\% \text { Foreign Nationals }{ }^{\mathrm{a}} \\
\text { SMD MP }\end{array}$ & & $\begin{array}{c}0.02 \\
(-0.04)\end{array}$ & & \\
\hline $\begin{array}{l}\text { Integration-relatedness of } \\
\text { manifesto content }{ }^{\mathrm{a}}\end{array}$ & $\begin{array}{l}0.07^{* *} \\
(-0.03)\end{array}$ & $\begin{array}{l}0.07 * * \\
(-0.03)\end{array}$ & $\begin{array}{c}0.04 \\
(-0.04)\end{array}$ & \\
\hline $\begin{array}{l}\text { Migrant-related } \\
\text { committee }\end{array}$ & $\begin{array}{c}0.92 * * * \\
(-0.25)\end{array}$ & $\begin{array}{c}0.93 * * * \\
(-0.26)\end{array}$ & $\begin{array}{l}0.75^{* *} \\
(-0.29)\end{array}$ & \\
\hline Manifesto $^{\mathrm{a}} *$ committee & & & $\begin{array}{c}0.04 \\
(-0.04)\end{array}$ & \\
\hline Migratory background & $\begin{array}{c}1.35^{* * *} \\
(-0.42)\end{array}$ & $\begin{array}{c}1.37 * * * \\
(-0.43)\end{array}$ & $\begin{array}{c}1.34 * * * \\
(-0.42)\end{array}$ & $\begin{array}{c}1.28^{* * *} \\
(-0.45)\end{array}$ \\
\hline \multicolumn{5}{|c|}{$\begin{array}{l}\text { Reference category: } S M D / \text { left- } \\
\text { wing/migrant-related committee }\end{array}$} \\
\hline $\begin{array}{l}\mathrm{PR} / \text { left-wing/ migrant } \\
\text {-related committee }\end{array}$ & & & & $\begin{array}{c}0.29 \\
(-0.36)\end{array}$ \\
\hline $\begin{array}{l}\text { SMD/ left-wing/ other } \\
\text { committee }\end{array}$ & & & & $\begin{array}{l}-0.79^{*} \\
(-0.46)\end{array}$ \\
\hline $\begin{array}{l}\mathrm{PR} / \text { left-wing/ other } \\
\text { committee }\end{array}$ & & & & $\begin{array}{l}-0.77^{*} \\
(-0.4)\end{array}$ \\
\hline $\begin{array}{l}\mathrm{PR} / \text { right-wing/ other } \\
\text { committee }\end{array}$ & & & & $\begin{array}{c}-3.00^{* * *} \\
(-1.16)\end{array}$ \\
\hline $\begin{array}{l}\mathrm{SMD} / \text { right-wing/ other } \\
\text { committee }\end{array}$ & & & & $\begin{array}{c}-1.47 * * \\
(-0.71)\end{array}$ \\
\hline $\begin{array}{l}\mathrm{PR} / \text { right-wing/ migrant } \\
\text {-related committee }\end{array}$ & & & & $\begin{array}{l}-0.94 \\
(-0.57)\end{array}$ \\
\hline $\begin{array}{l}\mathrm{SMD} / \text { right-wing/ migrant } \\
\text {-related committee }\end{array}$ & & & & $\begin{array}{c}-1.26 * * \\
(-0.64)\end{array}$ \\
\hline Intercept & $\begin{array}{c}-0.50^{*} \\
(-0.3)\end{array}$ & $\begin{array}{c}-0.52^{*} \\
(-0.3) \\
\end{array}$ & $\begin{array}{r}-0.41 \\
(-0.31) \\
\end{array}$ & $\begin{array}{l}0.65^{* *} \\
(-0.33) \\
\end{array}$ \\
\hline \multicolumn{5}{|l|}{ Zero-inflation logit model: } \\
\hline Total no. of PQs & $\begin{array}{c}-0.07 * * * \\
(-0.02)\end{array}$ & $\begin{array}{c}-0.07 * * * \\
(-0.02)\end{array}$ & $\begin{array}{c}-0.08 * * * \\
(-0.02)\end{array}$ & $\begin{array}{c}-0.07^{* * *} \\
(-0.02)\end{array}$ \\
\hline Intercept & $\begin{array}{c}2.41 * * * \\
(-0.38)\end{array}$ & $\begin{array}{c}2.40^{* * *} \\
(-0.38)\end{array}$ & $\begin{array}{c}2.44 * * * \\
(-0.38)\end{array}$ & $\begin{array}{c}2.24 * * * \\
(-0.4)\end{array}$ \\
\hline
\end{tabular}




\begin{tabular}{lcccc} 
Intercept alpha & $0.81 * * *$ & $0.81 * * *$ & $0.81 * * *$ & $0.80 * * *$ \\
& $(-0.21)$ & $(-0.21)$ & $(-0.2)$ & $(-0.2)$ \\
\hline $\mathrm{N}$ & 637 & 637 & 637 & 637 \\
Nonzero N & 110 & 110 & 110 & 110 \\
BIC & 928.47 & 934.78 & 934.4 & 951.91 \\
\hline
\end{tabular}

Note: Zero-inflated negative binomial regression models; Table entries show unstandardised coefficients with robust standard errors in parentheses; ${ }^{\mathrm{a}}$ variable centered at global mean; ${ }^{*} \mathrm{p}<0.10,{ }^{* *} \mathrm{p}<0.05, * * * \mathrm{p}<0.01$

Figure 2 visualises these effects. Based on model 1, the left-hand panel shows how the predicted number of integration-related PQs changes when the foreign national share increases from roughly two standard deviations below the mean up to two standard deviations above the mean. The predicted change is roughly one integration-related PQ. While this effect may seem substantially negligible, it is important to note that the mean number of integrationrelated PQs for our analysis is only at 0.83 . Based on model 2, the right-hand panel of figure 2 shows the marginal effect of being an SMD MP conditional on the local share of foreign nationals. As can be seen, the election mode does not interact with the size of the immigrant electorate in the constituency. Overall, these findings support the contention that MPs increase their number of integration-related PQs as the share of foreign nationals rises in their local constituencies where they were selected rather than elected $(\mathrm{H} 1)$.

Turning to the analysis of the party focus in MPs' integration-related PQs (H2a-c), Model 1 already provides evidence that the manifesto's integration-relatedness and the policy specialisation in migration-related committees shape MPs' parliamentary questioning behaviour considerably (in line with $\mathrm{H} 2 \mathrm{a}$ ). Model 3 examines the extent to which the committee effect is contingent on the integration-related content of the party manifesto by extending Model 1 by the interaction of both variables. 

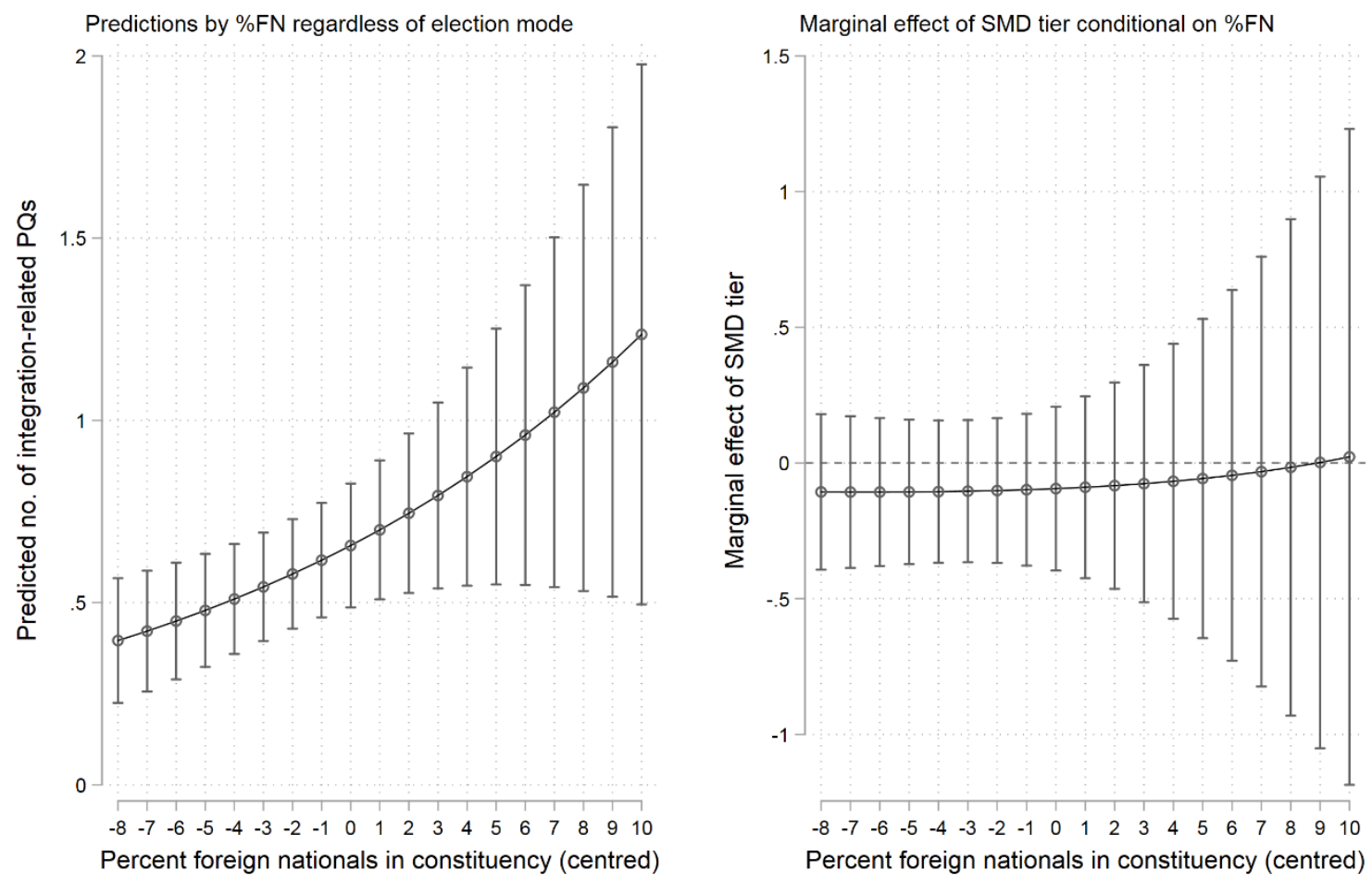

Figure 2. MPs' integration-related PQs in response to local demands with $95 \%$ confidence intervals

The coefficient for migration-related committee indicates that the effect of committee membership remains positive and statistically significant. Moreover, the interaction term indicates that as the integration-relatedness of the manifesto rises, so does the effect of migration-related committee. The calculated joint significance of the interaction term and migration-related committee is at $\mathrm{p}<0.01$. Estimating the marginal effects of the committee membership conditional on the manifesto's integration-relatedness (see the left-hand panel of Figure 3) indicates further that the committee effect is only noticeable if the centred manifesto scale takes values higher than -2 (10 on the non-centred scale). While right-wing MPs (CDU/CSU and FDP) fall below, left-wing MPs are all above this threshold. Taken together, this suggests that the effect of migration-related committee membership depends on a higher degree of the manifesto's integration-relatedness (H2b). 

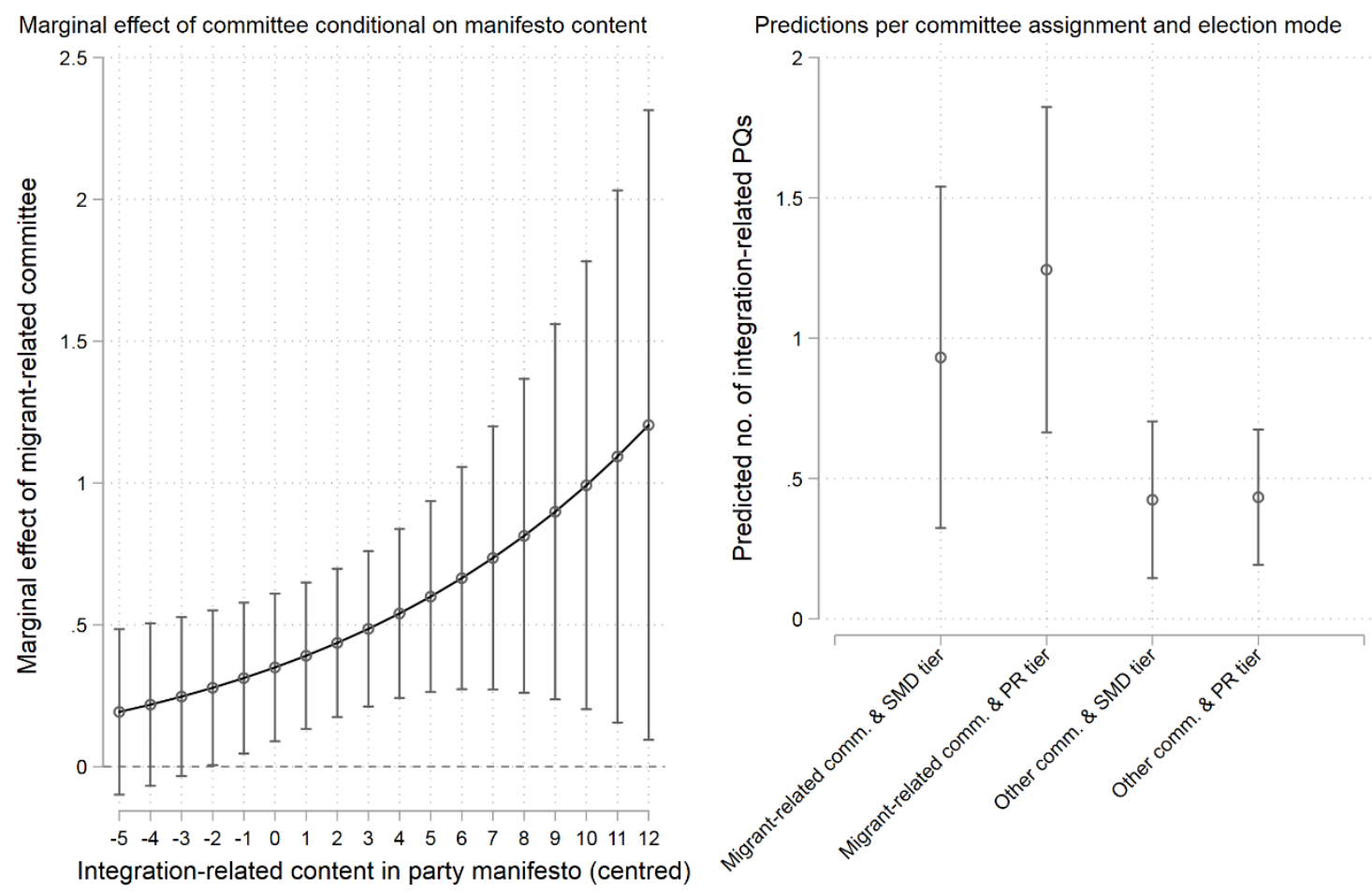

Figure 3. MPs' integration-related PQs in response to PPGs' demands with 95\% confidence intervals

In model 4 we intend to test whether the party focus in MPs' integration-related PQs further depends on their election in the PR tier $(\mathrm{H} 2 \mathrm{c})$. For this purpose, we create a three-way interaction between SMD MP, migration-related committee and their affiliation with a leftwing as opposed to a right-wing party ${ }^{7}$. Since this regression table is an unwieldy format to assess the model coefficients, we direct the reader to the visualisation of the predicted counts of integration-related PQs, shown in the right panel of Figure 3. In this visualisation, the left/right PPG distinction is held at 'left-wing', while MPs committee assignments and election modes vary. As can be seen, the effect of the committee assignment does not vary greatly between MPs' elected in the PR and SMD tier and the confidence intervals are widely overlapping. Therefore, H2c cannot be falsified based on this empirical evidence.

Furthermore, in all four models it is found that MPs of immigrant-origin are more likely to ask integration-related questions, corroborating findings from previous studies. However, the 
empirical evidence indicates that descriptive representation is only part of the story of immigrants' substantive representation.

Three major findings can be summarised from this analysis. First, MPs tend to ask more integration-related PQs the more foreign nationals reside in local constituencies where they were selected to run as district candidates. Second, they ask more of such questions when they sit on committees more likely to deal with matters of immigrants' integration as representatives of parties that make a commitment to improving the living conditions of disadvantaged immigrant groups in their manifestos. Third, these relationships seem to exist irrespective of MPs' own national backgrounds and regardless of whether they were elected under SMD or PR electoral rules in Germany's mixed-member system.

Our results are robust to different modelling strategies, which are presented in the online appendix A4.

\section{Concluding remarks}

Western representative democracies face new challenges due to the pressures of large-scale immigration creating multi-ethnic societies (Bird et al. 2011). Drawing on principal-agent models of democratic representation, this paper examines how institutional factors shape MPs' responsiveness to the disadvantages that immigrants and their descendants face in German society. Arguing that the role of native MPs has been underappreciated in previous research, we conceptualise MPs irrespective of their own national backgrounds as delegates who act based on the instructions of their most important principals: local constituents, party selectorates and PPGs. This analytical perspective constitutes a contrast to the "politics of 
presence' approach, which sees MPs rather as trustees whose conscience and personal experiences determine their legislative behaviour (Mansbridge 1999; Phillips 1995). However, we do not seek to contradict previous work based on the trustee conception. Rather, we argue that in order to improve our knowledge of the political representation of disadvantaged immigrant groups, it is fruitful to investigate relevant phenomena through a variety of analytical perspectives.

Drawing on a new dataset which includes a corpus of all 20,130 parliamentary questions (PQs) tabled by individual MPs in the $17^{\text {th }}$ Bundestag, we find that the demands of MPs' principals shape profoundly the substantive representation of disadvantaged immigrant groups in PQs. Moreover, the German institutional context, which confronts MPs with mixed electoral rules, a localised candidate selection process and tightly organised PPGs, allows us further to derive hypotheses about the behavioural consequences of these institutional features and to what extent they outperform each other. Putting these hypotheses to the test, our empirical results provide little support for the idea that differences in electoral rules shape immigrants' substantive representation in MPs' parliamentary questions. However, our findings do suggest, first, that MPs' responsiveness to local concentrations of immigrantorigin citizens hinges on a localised candidate selection method. Second, their responsiveness to the demands of national party bodies to speak on behalf of disadvantaged immigrant groups is a consequence of tightly organised PPGs in the Bundestag.

Taken together, our study makes therefore two important contributions to the literature. First, it shows that our understanding of the substantive representation of immigrant-origin minorities can be advanced by conceptualising MPs irrespective of their national backgrounds as delegates of principals inside and outside parliament. Second, this paper outlines also the limits of institutional explanations given the finding that candidate selection rules and 
legislative organisation are found to outperform electoral rules in their effects on immigrants' substantive representation in MPs' parliamentary questions.

Thus, future research should recognise more strongly the role of native MPs and the factors that affect their legislative behaviour. As long as different immigrant groups remain descriptively underrepresented in national legislatures, native MPs remain the most important vessel for this group's substantive representation. In other words, more research is needed to better understand MPs' legislative behaviour irrespective of their national backgrounds.

In this regard, our study of the German case is a first step. Comparative research would be a valuable extension to the present study in order to deepen our understanding of the consequences of candidate selection and legislative organisation for substantive representation across different electoral system regimes. Moreover, future research may also include other characteristics of MPs' institutional environments. For example, in many party-centred electoral systems national MPs pursue local political careers simultaneously (Fernandes et al. 2018; Russo 2011) or are subject to powerful local party branches in other ways (Tavits 2011). These factors can have the effect that MPs remain responsive to the demands of local concentrations of immigrant-origin citizens despite party-centred electoral rules. Given its parsimony, principal-agent theory should be a useful tool to explore the consequences of these factors in other parliamentary democracies, as well. In this light, the present contribution should be understood as a point of departure for future studies interested in the political representation of immigrants and their descendants in Western democracies. 


\section{References}

Alba, Richard, and Nancy Foner (2015). Strangers No More: Immigration and the Challenges of Integration in North America and Western Europe. Princeton: Princeton University Press.

Alonso, Sonia, and Saro Claro da Fonseca (2012). 'Immigration, left and right', Party Politics, 18:6, 865-884.

Aydemir, Nermin, and Rens Vliegenthart (2016). "“Minority Representatives” in the Netherlands: Supporting, Silencing or Suppressing?', Parliamentary Affairs , 69:1, 7392.

Bailer, Stefanie (2011). 'People's voice or information pool? The role of, and reasons for, parliamentary questions in the Swiss parliament', Journal of Legislative Studies, 17:3, $302-314$

Bailer, Stefanie (2017). 'To use the whip or not: Whether and when party group leaders use disciplinary measures to achieve voting unity', International Political Science Review, $39: 2,163-177$.

Bailer, Stefanie, and Tamaki Ohmura (2018). 'Exploring, Maintaining, and Disengaging-The Three Phases of a Legislator's Life', Legislative Studies Quarterly, 43:3, 493-520.

Bird, Karen, Thomas Saalfeld, and Andreas M Wüst (2011). 'Ethnic Diversity, Political Participation and Representation: a Theoretical Framework', in Karen Bird, Thomas Saalfeld,and Andreas M Wüst (eds.), The Political Representation of Immigrants and Minorities. Voters, Parties and Parliaments in Liberal Democracies, vol. London and New York: Routledge, 1-22.

Bischof, Daniel (2017). 'New graphic schemes for Stata: plotplain and plottig', Stata Journal, 
$17: 3,748-759$.

Bloemraad, Irene, and Karen Schönwälder (2013). 'Immigrant and Ethnic Minority Representation in Europe: Conceptual Challenges and Theoretical Approaches', West European Politics, 36:3, 564-579.

Bowler, Shaun, David M Farrell, and Richard S Katz (1999). 'Party Cohesion, Party Discipline, and Parliaments', in Shaun Bowler,David M Farrell,and Richard S Katz (eds.), Party Discipline and Parliamentary Government, vol. Columbus: Ohio State University Press, 3-22.

Bundeswahlleiter (2013). ‘5,8 Millionen Deutsche mit Migrationshintergrund sind wahlberechtigt', https://www.bundeswahlleiter.de/de/bundestagswahlen/BTW_BUND_13/presse/W1301 3_Wahlberechtigte_Migrationshintergrund.html (Accessed March 9, 2016).

Cameron, Adrian Colin, and Pravin K Trivedi (2013). Regression analysis of count data. Cambridge: Cambridge University Press.

Carey, John M (2009). Legislative Voting and Accountability. Cambridge: Cambridge University Press.

Carey, John M, and Matthew Soberg Shugart (1995). 'Incentives to cultivate a personal vote: A rank ordering of electoral formulas', Electoral Studies, 14:4, 417-439.

Converse, Philip E, and Roy Pierce (1986). Political Representation in France. Cambridge: MA: Belknap Press.

Cox, Gary W, and Mathew D McCubbins (2007). Legislative Leviathan. Party Government in the House. 2nd ed. Cambridge: Cambridge University Press. 
Crisp, Brian F (2007). 'Incentives in Mixed-Member Electoral Systems: General Election Laws, Candidate Selection Procedures, and Cameral Rules', Comparative Political Studies, 40:12, 1460-1485.

Dahl, Robert A (1971). Polyarchy: Participation and Opposition. New Haven: Yale University Press.

Damgaard, Erik (1995). 'How Parties control Committee Members', in Herbert Döring (ed.), Parliaments and Majority Rule in Western Europe, vol. Frankfurt am Main: Campus Verlag, 308-324.

Depauw, Sam, and Shane Martin (2009). 'Legislative Party Discipline and Cohesion in Comparative Perspective', in Daniela Giannetti and Kenneth R Benoit (eds.), Intra-Party Politics and Coalition Governments in Parliamentary Democracies, vol. London: Routledge, 103-120.

Detterbeck, Klaus (2016). 'Candidate Selection in Germany: Local and Regional Party Elites Still in Control?', American Behavioral Scientist, 60:7, 837-52.

Die Beauftragte der Bundesregierung für Migration, Flüchtlinge und Integration (2016). 11 . Bericht der Beauftragten der Bundesregierung für Migration, Flüchtlinge und Integration - Teilhabe, Chancengleichheit und Rechtsentwicklung in der Einwanderungsgesellschaft Deutschland.

Fernandes, Jorge M, Cristina Leston-Bandeira, and Carsten Schwemmer (2018). 'Election proximity and representation focus in party-constrained environments', Party Politics, $24: 6,674-685$.

Ferrara, Federico, Erik S Herron, and Misa Nishikawa (2005). Mixed Electoral Systems. Contamination and its Consequences. New York: Palgrave Macmillan. 
Gallagher, Michael (1988). 'Introduction', in Michael Gallagher and Michael Marsh (eds.), Candidate Selection in Comparative Perspective. The Secret Garden of Politics, vol. London: Sage, 1-19.

Hainmueller, Jens, and Holger Lutz Kern (2008). 'Incumbency as a source of spillover effects in mixed electoral systems: Evidence from a regression-discontinuity design', Electoral Studies, 27:2, 213-227.

Ismayr, Wolfgang (1992). Der Deutsche Bundestag. Funktionen - Willensbildung Reformansätze. Opladen: Leske and Budrich.

Ismayr, Wolfgang (2012). Der Deutsche Bundestag. Wiesbaden: VS Verlag für Sozialwissenschaften.

Karlsen, Rune, and Hanne M Narud (2013). 'Nominations, Campaigning and Representation: How the Secret Garden of Politics Determines the Style of Campaigning and Roles of Representation', in Peter Essaiasson and Hanne M Narud (eds.), Between-Election Democracy. The Representative Relationship after Election Day, vol. Colchester: ECPR Press, 77-101.

Manow, Philip (2013). 'Mixed Rules, Different Roles? An Analysis of the Typical Pathways into the Bundestag and of MPs' Parliamentary Behaviour', The Journal of Legislative Studies, 19:3, 287-308.

Mansbridge, Jane (1999). 'Should Blacks Represent Blacks and Women Represent Women? A Contingent "Yes"', The Journal of Politics, 61:03, 628-657.

Martin, Shane (2011a). 'Using Parliamentary Questions to Measure Constituency Focus: An Application to the Irish Case', Political Studies, 59:2, 472-488.

Martin, Shane (2011b). 'Parliamentary Questions, the Behaviour of Legislators, and the 
Function of Legislatures: An Introduction', The Journal of Legislative Studies, 17:3, $259-270$.

Martin, Shane (2014). 'Why electoral systems don't always matter: The impact of “megaseats" on legislative behaviour in Ireland', Party Politics, 20:3, 467-79.

Miller, Bernhard, and Christian Stecker (2008). 'Consensus by Default? Interaction of Government and Opposition Parties in the Committees of the German Bundestag.', German Politics, 17:3, 305-322.

Mitchell, Paul (2000). 'Voters and their representatives: electoral institutions and delegation in parliamentary democracies', European Journal of Political Research, 37, 335-351.

Moser, Robert G, and Ethan Scheiner (2012). Electoral Systems and Political Context. How the Effects of Rules Vary Across New and Established Democracies. Cambridge: Cambridge University Press.

Müller, Wolfgang C (2000). 'Political parties in parliamentary democracies: making delegation and accountability work', European Journal of Political Research, 37:3, 30933.

OECD, and EU (2015). Indicators of Immigrant Integration 2015: Settling In. Paris: OECD Publishing.

Phillips, Anne (1995). The Politics of Presence. Oxford: Clarendon Press.

Pitkin, Hanna Fenichel (1967). The Concept of Representation. Berkeley: University of California Press.

Preece, Jessica Robinson (2014). 'How The Party Can Win in Personal Vote Systems: The “Selectoral Connection" and Legislative Voting in Lithuania', Legislative Studies 
Quarterly, 39:2, 147-167.

Proksch, Sven-Oliver, and Jonathan Slapin (2015). The Politics of Parliamentary Debate. Cambridge: Cambridge University Press.

Rahat, Gideon, and Reuven Y Hazan (2001). 'Candidate selection methods - An analytical framework', Party Politics, 7:3, 297-322.

Rozenberg, Olivier, and Shane Martin (2011). 'Questioning Parliamentary Questions', The Journal of Legislative Studies, 17:3, 394-404.

Russo, Federico (2011). 'The Constituency as a Focus of Representation: Studying the Italian Case through the Analysis of Parliamentary Questions', The Journal of Legislative Studies, 17:3, 290-301.

Russo, Federico, and Matti Wiberg (2010). 'Parliamentary Questioning in 17 European Parliaments: Some Steps towards Comparison', The Journal of Legislative Studies, 16:2, $215-232$.

Saalfeld, Thomas (2011). 'Parliamentary Questions as Instruments of Substantive Representation: Visible Minorities in the UK House of Commons, 2005-10', The Journal of Legislative Studies, 17:3, 271-289.

Saalfeld, Thomas, and Daniel Bischof (2013). 'Minority-ethnic MPs and the substantive representation of minority interests in the house of commons, 2005-2011', Parliamentary Affairs, 66:2, 305-328.

Saalfeld, Thomas, and Kaare W Strøm (2014). 'Political Parties and Legislators', in Shane Martin,Thomas Saalfeld, and Kaare Strøm (eds.), Oxford Handbook of Legislative Studies, vol. Oxford: Oxford University Press, 371-398. 
Shugart, Matthew, Melody Ellis Valdini, and Kati Suominen (2005). 'Looking for locals: Voter information demands and personal vote-earning attributes of legislators under proportional representation', American Journal of Political Science, 49:2, 437-449.

Sieberer, Ulrich (2006). 'Party unity in parliamentary democracies: A comparative analysis', The Journal of Legislative Studies, 12:2, 150-178.

Strøm, Kaare (1997). 'Rules, Reasons and Routines: Legislative Roles in Parliamentary Democracies', in Wolfgang C Müller and Thomas Saalfeld (eds.), Members of Parliament in Western Europe: Roles and Behaviour, vol. London: Frank Cass, 155174.

Strøm, Kaare (1998). 'Parliamentary Committes in European Democracies', Journal of Legislative Studies, 4:1, 21-59.

Strøm, Kaare, and Wolfgang C Müller (1999). 'Political Parties and Hard Choices', in Wolfgang C Müller and Kaare Strøm (eds.), Policy, Office or Votes? How Political Parteis in Western Europe Make Hard Decisions, vol. Cambridge: Cambridge University Press, 1-35.

Swain, Carol (1993). Black Faces, Black Interests - The Representation of African Americans in Congress. London: Harvard University Press.

Tavits, Margit (2011). 'Power within Parties: The Strength of the Local Party and MP Independence in Postcommunist Europe', American Journal of Political Science, 55:4, 923-936.

Volkens, Andrea et al. (2015). The Manifesto Data Collection. Manifesto Project (MRG / CMP / MARPOR). Version 2015a. Berlin: Wissenschaftszentrum Berlin für Sozialforschung (WZB). 
Wüst, Andreas M (2011). 'Migrants as parliamentary actors in Germany', in Karen Bird,Thomas Saalfeld,and Andreas M Wüst (eds.), The Political Representation of Immigrants and Minorities. Voters, Parties and Parliaments in Liberal Democracies, vol. London and New York: Routledge, 250-65.

Wüst, Andreas M (2014a). 'A Lasting Impact? On the Legislative Activities of Immigrantorigin Parliamentarians in Germany', Journal of Legislative Studies,May, 37-41.

Wüst, Andreas M (2014b). 'Immigration into Politics: Immigrant-origin Candidates and Their Success in the 2013 Bundestag Election', German Politics \& Society, 32:3, 1-15.

Wüst, Andreas M (2016). 'Incorporation beyond Cleavages? Parties, Candidates and Germany’s Immigrant-Origin Electorate', German Politics, 25:2, 414-432. 


\section{Notes}

${ }^{1}$ If dual candidates are entitled to seats in both electoral tiers, they are automatically considered elected in the SMD tier and the PR tier seat will be allocated to the next candidate on the list. Due to the seat compensation mechanism between electoral tiers, parties' seat shares are not affected by these rules.

${ }^{2}$ All data used in this article, including the raw text of parliamentary questions, have been collected in the context of the PATHWAYS project (www.pathways.eu).

${ }^{3}$ Immigrant-related committees are labour and social affairs; education and research; family, elderly and women; domestic affairs; culture and media; human rights; economic development; petitions; and the investigation committee on the fascist terror of the 'Nationalsozialistischer Untergrund' (NSU).

${ }^{4}$ We chose a negative binomial model as diagnostics for a poisson model indicated overdispersion. Vuong tests further provided strong support for the use of zero-inflated mixture models.

${ }^{5}$ All figures shown in this paper were generated using the Stata scheme plotplain (Bischof 2017).

${ }^{6}$ We tested other specifications of the zero -inflation equation, including other variables used in the count equation. However, since these variables did not turn out to be significant and further increased the complexity of the models without improving their explanatory power indicated by a growing BIC value (Bayesian Information Criterion), we decided against their inclusion.

${ }^{7}$ Because MPs' election modes are strongly dependent on their party affiliation (almost all SMD MPs are either CDU/CSU or SPD), we would run into collinearity issues if we used the manifesto variable or party dummies in the interaction. Thus, we rely here on the rather simplistic left-right distinction. However, we would argue that it is reasonable to use this rather crude measure in interaction with the committee variable to capture PPGs' demands, because as Model 3 and the left-hand plot of Figure 3 have already shown, the committee effect is only significant for the three left-wing parties, such that it should make sense to compare the questioning behaviour of left-wing SMD and PR MPs who sit on migrant-related committees. 


\section{Appendix to paper}

\section{“MPs' Principals and the Substantive Representation of Disadvantaged Immigrant Groups"}

\section{A1: Measuring "integration-related" PQs}

The texts of German PQs were extracted from official online archives of the Bundestag using Python programming scripts. All files were available as PDF documents and were converted to raw text. Subsequently, several string matching procedures were used to isolate questions and subsequently match them with MP-level information.

The measurement goal is to identify PQs that raise attention to and demand the improvement of the living conditions of immigrants and their descendants. It is important to note that we do not intend to capture sceptical positions on the integration of immigrant-origin residents, i.e. content that relates to the protection of German national identity or expresses reservations against the integration of immigrants and multicultural society. In other words, our measure should not be mistaken as a measure of saliency or positioning on a pro- vs. anti-immigrant continuum.

The following two translated examples illustrate how parliamentary written questions are used by MPs in order to raise attention to and demand the improvement of the living conditions of immigrants and their descendants. 
„How does the government justify the Federal Office for Migration and Refugees recent announcement to cut the budget for integration courses in the light of the CDU, CSU and FPDs ' coalition agreements " plan to qualitatively and quantitatively upgrade those courses?" (PQ tabled by Aydan Özoğuz, SPD, May 7th 2010)

„,... how does the government want to ensure that the Federal Employment Office will bring residents with a migratory background into vocational training in similar proportions in their respective age groups as compared to Germans." (PQ tabled by Mechthild Rawert, SPD, March 18th 2011)

Ideally, in order to identify integration-related PQs, every single question in our corpus would be inspected qualitatively to determine whether it addresses immigrants' disadvantages in German society or not. As this is not feasible for over 20,000 questions we combine human and machine coding to identify integration-related PQs. The procedure involved four steps. In a first step we pre-defined a list of terms which have been manually extracted from the minutes of a parliamentary debate in which integration-related issues were discussed ${ }^{7}$. We also added other terms that we gathered from comprehensive qualitative inspections of the PQs. We then used this list of terms to filter the corpus. If, for example, a PQ includes the term "Migrationshintergrund" (German for "migratory background") or any other term in the list, this PQ would remain in our filtered corpus. A PQ without any terms on the list would be excluded from the corpus.

In a second step, we combined this filtered corpus with a random sample of remaining, nonfiltered PQs. Two hand coders were familiarised with our definition of substantive representation and then were asked to classify each question as either integration-related or 
not $^{7}$. The intercoder-reliability in form of Cohen's Kappa (Cohen 1960) between human coders was 0.79 . All coding disagreements were discussed and recoded after consensus accordingly. Additionally, from each validated question, our hand-coders again collected specific key terms which indicate that the question is integration-related. We updated the key term list accordingly.

In a third step we used the hand-coded corpus to test our updated key term list for the identification of integration-related PQs. By using the updated list of key terms ${ }^{7}, 82 \%$ of all questions in our validated corpus were classified correctly ${ }^{7}$. In a fourth step we applied our updated key term list to all 20,130 PQs, identifying a total of 869 potentially integrationrelated questions in the whole corpus.

One concern with key term-based textual analyses is its susceptibility to falsely capturing irrelevant documents (false positives), while at the same time failing to capture relevant documents (false negatives). In order to keep such bias at a minimum, we inspected in a final step all 869 positives qualitatively in order to discard false positives, which left us with a total of 544 PQs as a final measure of integration-related PQs. This amounts to $2.7 \%$ of all PQs in our corpus.

Concerns regarding false negatives cannot be quantified to the same extent, but we are confident that this does not pose too great a problem to our measurement, given that we have included a random subset of the unfiltered corpus in our validation approach in step 2. Nevertheless, to be fair, we cannot completely rule out that the captured number of integration-related PQs constitutes an underestimation of the real number of integrationrelated PQs in the analysed text corpus. 


\section{A2: Final term dictionary to identify questions}

abgeschoben, abschiebehaftbedingungen, abschiebestopps, abschiebung, abschiebungen, altübersiedler, aufenthaltstitel, antidiskriminierungsrichtlinie, antidiskriminierungsstelle, arbeitserlaubnis, aslybewerberleistungsbezug, assoziationsrecht, asyl, asylantrag, asylantragstellern, asylanträge, asylbewerber, asylbewerberinnen, asylbewerberleistungsbezug, asylbewerberleistungsgesetz, asylbewerberleistungsgesetzes, asylbewerberleistungsgestz, asylbewerberleisungsgesetz, asylbewerbern, asylbewerbers, asylblg, asylsuchende, asylsuchenden, asylsuchendenzahlen, asylsuchender, asylsystem, asylsystems, asylverfahren, asylverfahrenrichtlinie, asylverfahrensgesetz, asylverfahrensgesetzes, asylverfahrensrecht, asylverfahrensrichtlinie, asylverfahrungsgesetz, aufenthaltgesetz, aufenthaltsstatus, aufenthaltserlaubnis, aufenthaltserlaubnisse, aufenthaltserlaubnis, aufenthaltsgesetz, aufenthaltsgesetze, aufenthaltsgesetzes, aufenthaltsgestaltung, aufenthaltsgewährung, aufenthaltspapiere, aufenthaltsrecht, aufenthaltstitel, ausländer, ausländerbeschäftigungsrecht, ausländerförderung, ausländerjagdschein, ausländerzentralregister, ausländischer, aussiedler, balkanflüchtlinge, bleiberechtsregelung, bleibeberechtigung, bürgerkriegsflüchtlinge, bürgerkriegsflüchtlingen, diskriminierung, doppelstaatlers, drittstaatangehörige, drittstaatsangehörige, drittstaatsangehörigen, dublin-ii, dublinüberstellungsverfahren, ehegattennachzug, einbürgerung, einbürgerungstest, einbürgerungstests, einbürgerungsverhalten, eingebürgert, einreiseerlaubnis, einreisevisum, einwanderern, einwanderungsgruppen, eu-aufnahmerichtlinie, eu-aufnahmerichtlinien, fachkräfteanwerbung, familiennachzug, familienzusammenführung, familienzusammenführungsrichtlinen, familienzusammenführungsrichtlinie, flüchtlinge, flüchtlingen, flüchtlingselend, flüchtlingskonvention, flüchtlingslager, frontex, grenzsicherug, grenzübergangsstellen, herkunftsfamilie, herkunftsland, herkunftsstaaten, integration, integrationsansprüche, integrationsarbeit, integrationscoaching, integrationsfördernd, integrationsförderung, integrationsgipfel, integrationsherausforderungen, integrationskurs, integrationskursbeteiligung, integrationskurse, integrationskursen, integrationsleistung, integrationsleistungen, 
integrationsministerkonferenz, integrationspolitik, integrationspolitisch, integrationsprogramm, integrationsprogramms, integrationsprojekte, integrationssprachkursleiter, integrationstest, integrationsunwillig, integrationsverordnung, integriert, interkulturelle bildung, intgegrationsprojekte, islam, jugendintegrationskurse, jugendmigrationsdienst, jugendmigrationsdienstes, migranten, migrantinnen, migration, migrationsabkommen, migrationsbiographie, migrationshintergrund, migrationshintergrund, migrationshintergrundes, minderheitsangehoerige, minderheitsangehörige, immigranten, optionskind, optionskinder, optionspflicht, optionspflichtige, rassismus, resettlementprogramms, roma-minderheit, rückführungsabkommen, rückführungsentscheidungen, rücknahmeabkommen, rückübernahmeabkommen, rückübernahmeabkommens, rücküberstellung, sammelunterkünfte, sammelunterkünften, scheineheverdachts, scheineheverdachtsfälle, sprachförderung, sprachkurs, sprachkurse, sprachkursen, sprachtest, spätaussiedler, staatenlose, staatsangehörigkeit, staatsangehörigkeitsgesetz, staatsangehörigkeitsrecht, staatsbürgerschaft, visa, visagebühren, visapflicht, visavergabe, visum, visumantrags, visumanträge, visumbefreiung, visumfreiheit, visumgebühren, visums, visumsanträge, visumsbefreiung, visumsfreiheit, visumsgebühren, visumspflicht, visumverfahren, zugewandert, zuwanderer, zuwanderern, zuwanderung

\section{A3: Coding of party manifestos' integration relatedness}

Following previous work in the field, we measure the degree to which party manifestos contain claims of integrating immigrant-minorities into society (integration-relatedness) based on data from the comparative manifesto project (Alonso and Fonseca 2012; Wüst 2016; Volkens et al. 2015). Similarly to Alonso and Fonseca (2012) as well as Wüst (2016), we build an additive index based on the following items: positive values for per602 (national way of life: negative), per607 (multiculturalism: positive), per705 (favourable references to underprivileged minorities); and negative values for the items per601 (national way of life: 
positive) and per608 (multiculturalism: negative). However, in addition to these items and in difference to the cited literature, we also add positive values for the item per503 (Equality: positive). Including the equality item per503 takes into account that policy agendas with a focus on redistribution, equal opportunities and racial equality, tend to intersect "with the material and subjective aspirations of immigrant voters who generally find themselves socioeconomically disadvantaged or the objects of racial prejudice or social exclusion" (Messina 2007: 208). Thus, by including this item in the index, our measure comes closer to the running definition of immigrant-origin citizens' integration (see page 16 in the main article). Nevertheless, as a robustness check, we re-estimated the first three models of Table 2 shown in the main article using a more parsimonious index that excludes per503. As can be seen in the section on the robustness checks (robustness check 3 in this appendix file), results do not change considerably when per503 is considered or not. Based on our operationalisation, the five parties achieve the following scores in 2009:

\section{$\mathrm{CDU} / \mathrm{CSU} \quad 7.121$}

FDP $\quad 6.935$

SPD $\quad 16.894$

Greens $\quad 16.435$

The Left $\quad 24.910$ 


\section{A4: Robustness Checks}

As robustness checks, we refitted the models as standard negative binomial regression models on the whole sample of MPs (Robustness check 1) and on a reduced sample of MPs who have asked at least one PQ (Robustness check 2). Robustness check 3 replicates Models 1-3 from the main article using the same party manifesto coding as Wüst (2016) does.

Robustness Check 1 - Negative binomial regression models

\begin{tabular}{|c|c|c|c|c|}
\hline & $\begin{array}{c}\text { Model } 1 \\
\mathrm{~b} / \mathrm{se}\end{array}$ & $\begin{array}{c}\text { Model } 2 \\
\mathrm{~b} / \mathrm{se}\end{array}$ & $\begin{array}{c}\text { Model } 3 \\
\mathrm{~b} / \mathrm{se}\end{array}$ & $\begin{array}{c}\text { Model } 4 \\
\mathrm{~b} / \mathrm{se}\end{array}$ \\
\hline$\%$ Foreign Nationals ${ }^{a}$ & $\begin{array}{c}0.07 * * * \\
(0.02)\end{array}$ & $\begin{array}{l}0.06^{* *} \\
(0.03)\end{array}$ & $\begin{array}{c}0.07 * * * \\
(0.02)\end{array}$ & $\begin{array}{c}0.06 * * * \\
(0.02)\end{array}$ \\
\hline SMD MP & $\begin{array}{l}-0.20 \\
(0.26)\end{array}$ & $\begin{array}{l}-0.22 \\
(0.26)\end{array}$ & $\begin{array}{l}-0.20 \\
(0.26)\end{array}$ & \\
\hline $\begin{array}{l}\% \text { Foreign Nationals }{ }^{\mathrm{a}} * \\
\text { SMD MP: }\end{array}$ & & $\begin{array}{c}0.02 \\
(0.04)\end{array}$ & & \\
\hline $\begin{array}{l}\text { Integration-related } \\
\text { manifesto content }{ }^{\mathrm{a}}\end{array}$ & $\begin{array}{c}0.09 * * * \\
(0.02)\end{array}$ & $\begin{array}{c}0.09 * * * \\
(0.02)\end{array}$ & $\begin{array}{c}0.07 * * \\
(0.03)\end{array}$ & \\
\hline $\begin{array}{l}\text { Migrant-related } \\
\text { committee }\end{array}$ & $\begin{array}{c}1.08 * * * \\
(0.26)\end{array}$ & $\begin{array}{c}1.08 * * * \\
(0.26)\end{array}$ & $\begin{array}{c}0.99 * * * \\
(0.29)\end{array}$ & \\
\hline Manifesto $^{\text {a }} *$ committee & & & $\begin{array}{c}0.03 \\
(0.04)\end{array}$ & \\
\hline Migratory background & $\begin{array}{c}1.36^{* * * *} \\
(0.42)\end{array}$ & $\begin{array}{c}1.38 * * * \\
(0.43)\end{array}$ & $\begin{array}{c}1.35 * * * \\
(0.42)\end{array}$ & $\begin{array}{c}1.27 * * * \\
(0.39)\end{array}$ \\
\hline $\begin{array}{l}\text { Reference category: SMD/ } \\
\text { left-wing/ migrant-related } \\
\text { committee }\end{array}$ & & & & \\
\hline $\begin{array}{l}\mathrm{PR} / \text { left-wing/ migrant } \\
\text {-related committee }\end{array}$ & & & & $\begin{array}{l}-0.08 \\
(0.34)\end{array}$ \\
\hline $\begin{array}{l}\text { SMD/ left-wing/ other } \\
\text { committee }\end{array}$ & & & & $\begin{array}{l}-1.35^{* * *} \\
(0.46)\end{array}$ \\
\hline $\begin{array}{l}\mathrm{PR} / \text { left-wing/ other } \\
\text { committee }\end{array}$ & & & & $\begin{array}{c}-1.12 * * * \\
(0.38)\end{array}$ \\
\hline $\begin{array}{l}\mathrm{PR} / \text { right-wing/ other } \\
\text { committee }\end{array}$ & & & & $\begin{array}{c}-3.68 * * * \\
(1.09)\end{array}$ \\
\hline $\begin{array}{l}\text { SMD/ right-wing/ other } \\
\text { committee }\end{array}$ & & & & $\begin{array}{l}-2.29 * * * \\
(0.57)\end{array}$ \\
\hline $\begin{array}{l}\mathrm{PR} / \text { right-wing/ migrant } \\
\text {-related committee }\end{array}$ & & & & $\begin{array}{c}-1.36^{* *} \\
(0.63)\end{array}$ \\
\hline $\begin{array}{l}\mathrm{SMD} / \text { right-wing/ migrant } \\
\text {-related committee }\end{array}$ & & & & $\begin{array}{c}-2.11 * * * \\
(0.71)\end{array}$ \\
\hline
\end{tabular}




\begin{tabular}{lcccc}
\hline Total no. of PQs & $0.02 * * *$ & $0.02 * * *$ & $0.02 * * *$ & $0.02 * * *$ \\
Intercept & $(0.00)$ & $(0.00)$ & $(0.00)$ & $(0.00)$ \\
& $-2.74 * * *$ & $-2.73 * * *$ & $-2.67 * * *$ & $-0.87 * * *$ \\
& $(0.29)$ & $(0.29)$ & $(0.28)$ & $(0.31)$ \\
\hline \multirow{2}{*}{ Intercept alpha } & & & & \\
& $1.28 * * *$ & $1.28 * * *$ & $1.27 * * *$ & $1.17 * * *$ \\
$\mathrm{~N}$ & $(0.16)$ & $(0.16)$ & $(0.16)$ & $(0.17)$ \\
$\mathrm{BIC}$ & 637.00 & 637.00 & 637.00 & 637.00 \\
\hline
\end{tabular}

Note: Negative binomial regression models; Table entries show unstandardised coefficients with robust standard errors in parentheses; ${ }^{a}$ variable centred at global mean; ${ }^{*} \mathrm{p}<0.10,{ }^{* *} \mathrm{p}<0.05,{ }^{* * *} \mathrm{p}<0.01$

Robustness Check 2 - Negative binomial regression models only for MPs who asked at least one question

\begin{tabular}{|c|c|c|c|c|}
\hline & $\begin{array}{c}\text { Model } 1 \\
\text { b/se }\end{array}$ & $\begin{array}{c}\text { Model } 2 \\
\text { b/se }\end{array}$ & $\begin{array}{c}\text { Model } 3 \\
\text { b/se }\end{array}$ & $\begin{array}{c}\text { Model } 4 \\
\text { b/se }\end{array}$ \\
\hline$\%$ Foreign Nationals ${ }^{\mathrm{a}}$ & $\begin{array}{c}0.05 * * * \\
(0.02)\end{array}$ & $\begin{array}{l}0.04 * \\
(0.02)\end{array}$ & $\begin{array}{c}0.05 * * * \\
(0.02)\end{array}$ & $\begin{array}{c}0.05 * * * \\
(0.02)\end{array}$ \\
\hline SMD MP & $\begin{array}{l}-0.04 \\
(0.23)\end{array}$ & $\begin{array}{l}-0.05 \\
(0.24)\end{array}$ & $\begin{array}{l}-0.04 \\
(0.23)\end{array}$ & \\
\hline $\begin{array}{l}\% \text { Foreign Nationals }{ }^{\mathrm{a}} * \\
\text { SMD MP: }\end{array}$ & & $\begin{array}{c}0.02 \\
(0.04)\end{array}$ & & \\
\hline $\begin{array}{l}\text { Integration-related } \\
\text { manifesto content }{ }^{\mathrm{a}}\end{array}$ & $\begin{array}{c}0.02 \\
(0.02)\end{array}$ & $\begin{array}{c}0.02 \\
(0.02)\end{array}$ & $\begin{array}{c}0.03 \\
(0.03)\end{array}$ & \\
\hline $\begin{array}{l}\text { Migrant-related } \\
\text { committee }\end{array}$ & $\begin{array}{c}0.73 * * * \\
(0.22)\end{array}$ & $\begin{array}{c}0.73 * * * \\
(0.23)\end{array}$ & $\begin{array}{c}0.75 * * * \\
(0.27)\end{array}$ & \\
\hline Manifesto $^{\mathrm{a}} *$ committee & & & $\begin{array}{l}-0.00 \\
(0.04)\end{array}$ & \\
\hline Migratory background & $\begin{array}{c}1.11 * * * \\
(0.28)\end{array}$ & $\begin{array}{c}1.15 * * * \\
(0.29)\end{array}$ & $\begin{array}{c}1.12 * * * \\
(0.29)\end{array}$ & $\begin{array}{c}1.13 * * * \\
(0.28)\end{array}$ \\
\hline $\begin{array}{l}\text { Reference category: SMD/ } \\
\text { left-wing/ migrant-related } \\
\text { committee }\end{array}$ & & & & \\
\hline $\begin{array}{l}\mathrm{PR} / \text { left-wing/ migrant } \\
\text {-related committee }\end{array}$ & & & & $\begin{array}{l}-0.08 \\
(0.33)\end{array}$ \\
\hline $\begin{array}{l}\text { SMD/ left-wing/ other } \\
\text { committee }\end{array}$ & & & & $\begin{array}{l}-0.73^{*} \\
(0.43)\end{array}$ \\
\hline $\begin{array}{l}\mathrm{PR} / \text { left-wing/ other } \\
\text { committee }\end{array}$ & & & & $\begin{array}{c}-0.77 * * \\
(0.34)\end{array}$ \\
\hline $\begin{array}{l}\mathrm{PR} / \text { right-wing/ other } \\
\text { committee }\end{array}$ & & & & $\begin{array}{c}-2.58^{* *} \\
(1.06)\end{array}$ \\
\hline $\begin{array}{l}\text { SMD/ right-wing/ other } \\
\text { committee }\end{array}$ & & & & $\begin{array}{l}-1.01^{*} \\
(0.57)\end{array}$ \\
\hline $\mathrm{PR} /$ right-wing/ migrant & & & & 0.02 \\
\hline
\end{tabular}


-related committee

$\mathrm{SMD} /$ right-wing/ migrant

-related committee

Total no. of PQs

$0.08 * * *$

$0.08 * * *$

$0.08 * * *$

$0.07 * * *$

Intercept

(0.01)

$(0.01)$

$(0.01)$

$(0.01)$

$-1.91 * * *$

$-1.91 * * *$

$-1.92 * * *$

$-0.91 * * *$

$(0.21)$

$(0.21)$

$(0.23)$

(0.30)

Intercept alpha

$0.80 * * *$

$0.80 * * *$

$0.80 * * *$

$0.74 * * *$

\begin{tabular}{lcccc} 
& $(0.17)$ & $(0.17)$ & $(0.17)$ & $(0.18)$ \\
\hline $\mathrm{N}$ & 387.00 & 387.00 & 387.00 & 387.00 \\
$\mathrm{BIC}$ & 861.30 & 866.84 & 867.25 & 878.21 \\
\hline
\end{tabular}

Note: Negative binomial regression models; Table entries show unstandardised coefficients with robust standard errors in parentheses; ${ }^{\mathrm{a}}$ variable centred at global mean; ${ }^{*} \mathrm{p}<0.10,{ }^{* *} \mathrm{p}<0.05,{ }^{* * *} \mathrm{p}<0.01$

Robustness Check 3 - Zero-inflated negative binomial regression models with alternative manifesto coding

\begin{tabular}{|c|c|c|c|}
\hline & $\begin{array}{l}\text { Model } 1 \\
\text { b/se }\end{array}$ & $\begin{array}{c}\text { Model } 2 \\
\mathrm{~b} / \mathrm{se}\end{array}$ & $\begin{array}{c}\text { Model } 3 \\
\text { b/se }\end{array}$ \\
\hline \multicolumn{4}{|l|}{$\begin{array}{l}\text { Negative binomial } \\
\text { count model: }\end{array}$} \\
\hline$\%$ Foreign Nationals $^{\mathrm{a}}$ & $\begin{array}{l}0.06 * * \\
(0.02)\end{array}$ & $\begin{array}{c}0.06 \\
(0.03)\end{array}$ & $\begin{array}{l}0.06 * * \\
(0.02)\end{array}$ \\
\hline SMD MP & $\begin{array}{l}-0.09 \\
(0.26)\end{array}$ & $\begin{array}{l}-0.10 \\
(0.26)\end{array}$ & $\begin{array}{l}-0.08 \\
(0.26)\end{array}$ \\
\hline $\begin{array}{l}\% \text { Foreign Nationals }{ }^{\mathrm{a}} * \\
\text { SMD MP: }\end{array}$ & & $\begin{array}{c}0.01 \\
(0.04)\end{array}$ & \\
\hline $\begin{array}{l}\text { Integration-related } \\
\text { manifesto content }{ }^{\mathrm{a}}\end{array}$ & $\begin{array}{l}0.27 * \\
(0.14)\end{array}$ & $\begin{array}{l}0.28^{*} \\
(0.14)\end{array}$ & $\begin{array}{c}0.14 \\
(0.19)\end{array}$ \\
\hline $\begin{array}{l}\text { Migrant-related } \\
\text { committee }\end{array}$ & $\begin{array}{c}0.95^{* * *} \\
(0.26)\end{array}$ & $\begin{array}{c}0.96^{* * *} \\
(0.27)\end{array}$ & $\begin{array}{c}0.78 * * * \\
(0.29)\end{array}$ \\
\hline Manifesto $^{\text {a }} *$ committee & & & $\begin{array}{c}0.21 \\
(0.22)\end{array}$ \\
\hline Migratory background & $\begin{array}{l}1.31 * * * \\
(0.42)\end{array}$ & $\begin{array}{c}1.32 * * * \\
(0.43)\end{array}$ & $\begin{array}{c}1.29 * * * \\
(0.42)\end{array}$ \\
\hline Intercept & $\begin{array}{l}-0.41 \\
(0.30)\end{array}$ & $\begin{array}{l}-0.42 \\
(0.31)\end{array}$ & $\begin{array}{l}-0.31 \\
(0.31)\end{array}$ \\
\hline \multicolumn{4}{|l|}{$\begin{array}{l}\text { Zero-inflation logit } \\
\text { model: }\end{array}$} \\
\hline Total no. of PQs & $\begin{array}{c}-0.08 * * * \\
(0.02)\end{array}$ & $\begin{array}{c}-0.08 * * * \\
(0.02)\end{array}$ & $\begin{array}{c}-0.08 * * * \\
(0.02)\end{array}$ \\
\hline Intercept & $\begin{array}{c}2.54 * * * \\
(0.35)\end{array}$ & $\begin{array}{c}2.53 * * * \\
(0.35)\end{array}$ & $\begin{array}{c}2.57 * * * \\
(0.35)\end{array}$ \\
\hline Intercept alpha & $\begin{array}{c}0.82 * * * \\
(0.20)\end{array}$ & $\begin{array}{c}0.82 * * * \\
(0.20)\end{array}$ & $\begin{array}{c}0.81 * * * \\
(0.20)\end{array}$ \\
\hline $\mathrm{N}$ & 637 & 637 & 637 \\
\hline
\end{tabular}




\begin{tabular}{lccc} 
Nonzero N & 110 & 110 & 110 \\
BIC & 929.98 & 936.36 & 935.71 \\
\hline
\end{tabular}

Note: Zero-inflated negative binomial regression models; Table entries show unstandardised coefficients with robust standard errors in parentheses; ${ }^{\text {a }}$ variable centred at global mean; ${ }^{*} \mathrm{p}<0.10, * * \mathrm{p}<0.05, * * * \mathrm{p}<0.01$

Appendix sources

Alonso, Sonia, and Saro Claro da Fonseca (2012). 'Immigration, left and right', Party Politics, 18:6, 865-884.

Cohen, Jacob (1960). 'A coefficient of agreement for nominal scales', Educational and Psychological Measurement, 20:1, 37-46.

Deutscher Bundestag (2010). 'Stenografischer Bericht. 68. Sitzung (17/68). 28 October 2010.', http://dip21.bundestag.de/dip21/btp/17/17068.pdf.

Grimmer, Justin, and Brandon M Stewart (2013). 'Text as Data: The Promise and Pitfalls of Automatic Content Analysis Methods for Political Texts', Political Analysis, 1:1-31.

Messina, Anthony M (2007). The Logics and Politics of Post-WWII Migration to Western Europe. Cambridge: Cambridge University Press.

Volkens, Andrea et al. (2015). The Manifesto Data Collection. Manifesto Project (MRG / CMP / MARPOR). Version 2015a. Berlin: Wissenschaftszentrum Berlin für Sozialforschung (WZB).

Wüst, Andreas M (2016). 'Incorporation beyond Cleavages? Parties, Candidates and Germany’s Immigrant-Origin Electorate', German Politics, 25:2, 414-432. 


\section{Aufsatz 4}

Lucas Geese (2019) Immigration-related Speechmaking in a Partyconstrained Parliament: Evidence from the 'Refugee Crisis' of the 18th German Bundestag (2013-2017), German Politics, https://doi.org/10.1080/09644008.2019.1566458

Das folgende Manuskript ist der Post-print des Aufsatzes, der von der Redaktion der Fachzeitschrift German Politics (ISSN: 0964-4008) zur Publikation angenommen wurde. 


\title{
Immigration-related speechmaking in a party-constrained parliament: evidence from the 'refugee crisis' of the 18th German Bundestag (2013-2017)
}

\author{
The 18th Bundestag term was marked by a high salience of the refugee and \\ asylum issue dominating the political agenda. Taking this context as a case study, \\ this paper asks which factors make legislators talk about immigration on the \\ parliamentary floor. Three different literatures provide different answers to this \\ question. A first literature highlights that immigrant-origin legislators with a \\ visible background may have intrinsic motives to talk about immigration. A \\ second literature raises attention to legislators' personal vote-seeking incentives \\ to talk about immigration when the issue is electorally decisive. Contrary to these \\ first two literatures, a third literature posits that legislative debates do not provide \\ legislators the leeway to follow individual motives on the parliamentary floor, but \\ that parliamentary party groups (PPGs) control access to the parliamentary floor \\ and thus follow own strategies when allocating floor time to speak about the \\ immigration issue. To examine this puzzle of competing expectations, a corpus of \\ more than 10,000 speeches is leveraged, utilising a structural topic model, a \\ novel method of quantitative text analysis. Results suggest that legislators' \\ speech attention to the refugee and asylum issue in the 18th Bundestag was \\ mainly shaped by PPG specific factors rather than by their individual motives.
}

Keywords: immigration, representation, speechmaking, Bundestag, legislators

\section{Introduction}

Over the past few decades, immigration has become a highly salient and electorally decisive policy issue in a number of Western European democracies (Green-Pedersen and Otjes 2017). Investigating political attention to the immigration issue is therefore a fundamental topic in contemporary European political science. Our understanding of the phenomenon has benefited from a number of recent contributions analysing the determinants of issue attention to immigration in party manifestos (Kortmann and Stecker 2017; Ruedin and Morales 2017; Green-Pedersen and Otjes 2017). Less is known, by contrast, about the politics of immigration-related speechmaking (but see 
Blätte and Wüst 2017). However, as speeches on the floor provide legislators and their parties a publicly exposed stage to communicate issue priorities and policy positions, they are important tools of political communication and representation (Proksch and Slapin 2015; Bäck and Debus 2016; Bächtiger 2014). By drawing attention to certain policy issues while deemphasising others in their speeches, representatives can publicly demonstrate that they are responsive to current problem pressures and voters' issue priorities (Budge and Farlie 1983; Spoon and Klüver 2014; Green-Pedersen and Otjes 2017). This raises the question of why some legislators draw attention to immigration in their speeches while others do not, despite the overall growing salience of the issue.

The present article pursues this question by focussing on the $18^{\text {th }}$ German Bundestag (2013-17), a legislative term during which the issue of refugees and asylum became a hot topic in the course of the European refugee crisis. Taking this term as a crucial case study, this article attempts to contrast individual with collective explanations of legislative behaviour. Two literatures raise attention to individual motives as potential drivers of speechmaking. First, intrinsic motives flowing from the personal experiences of legislators who themselves or whose parents have a migratory history may lead to more speech attention to the issue of refugees and asylum as compared to other legislators (Searing 1994; Searing 1991; Wüst 2014a; Saalfeld and Bischof 2013; Saalfeld 2011; Blätte and Wüst 2017). A second literature argues that, depending on their individual re-election contexts, legislators have extrinsic motives to talk about policy issues that dominate the political agenda (Mayhew 1974; Carey and Shugart 1995; Carey 2009; Zittel and Gschwend 2008; André, Depauw, and Martin 2015).

However, insofar as parliamentary democracies are analysed, we need to be careful not to underestimate the role of collective parliamentary party groups (PPGs) in 
legislative speechmaking. In this regard, a third literature casts doubts as to whether speeches on the floor provide legislators the necessary leeway to express individual concerns when highly salient policy issues are debated. In a nutshell, the argument is that in most parliaments PPG leaderships control not just the plenary thematic agenda but decide also who speaks about which topic (Proksch and Slapin 2015; Bäck, Debus, and Müller 2014; Bäck and Debus 2018; Bäck and Debus 2016; Cox 2006). Therefore, legislators may be often constrained in following individual motives, but are rather forced to follow collective motives on the parliamentary floor, regardless of whether they are, as individuals, intrinsically or extrinsically motivated.

Empirically, the study is based on a novel dataset containing over 10,000 legislative speeches held in the $18^{\text {th }}$ Bundestag. To measure speech attention to the refugee and asylum issue, it utilises a new method of quantitative text analysis: structural topic models (STM) (Roberts et al. 2014). Results indicate that during the most recent German immigration shock, PPGs' organisational capacities and their roles as gatekeepers to the parliamentary floor were far stronger determinants of such speechmaking than legislators' individual motives.

\section{What makes legislators take the parliamentary floor to speak about immigration?}

Before the theoretical framework is presented, it is necessary to clarify what immigration-related speechmaking means conceptually for this study. Importantly, this paper is not about the question of representing the interests of long-term citizens of immigrant-origin with full voting rights. Rather, it is about speech attention to the issue of refugees and asylum, referring to a group of people who are widely considered to remain in Germany only for a limited period of time and who, as non-citizens, have no opportunity to partake in the electoral process. Put differently, immigrants and 
specifically refugees and asylum seekers are conceptualised in this study to be the content of political representation, rather than a social group meant to be politically represented. To be sure, many immigrant-origin citizens may have a specific interest in migration-related issues, including the issue of refugees and asylum. Thus, scholars often look for migration-related content in parliamentary behaviour when examining questions of the substantive representation of immigrant-origin citizens (Blätte and Wüst 2017; Wüst 2014a; Saalfeld and Bischof 2013; Saalfeld 2011). However, migration-related policy preferences may differ across different groups of immigrantorigin citizens (Heath et al. 2013, chap. 4). In Germany, for example, Goerres and coauthors show that the group of so-called 'ethnic' Germans expressed to a large extent sceptical stances regarding the influx of refugees and asylum seekers during the 2017 Bundestag election campaign (Goerres, Mayer, and Spies 2018). Therefore, although there may be some overlap between immigrant-origin citizens' interests and attention to the issue of refugee and asylum, it is nevertheless instrumental to refer to these as two different dimensions of immigration politics, with the latter being the focus of this study.

\section{Legislators' immigrant backgrounds and speech attention to immigration}

The first individual factor considered to affect legislative speechmaking relates to legislators' intrinsic motives shaped by their personal backgrounds. 'Motivational' parliamentary role theory assumes that legislators are purposive actors who pursue their exogenously (career-related) and endogenously (emotionally) conditioned individual goals in parliament (Blomgren and Rozenberg 2012, 21-27; Searing 1991). Unless they have the institutional responsibility to fulfil a 'leadership role' like whip, legislators are thought to be relatively free to choose from different pre-defined 'preference roles' (Searing 1991, 1249-50). Among those are 'policy advocates' who mainly seek to 
influence government policy, 'constituency members' who choose to concentrate their legislative activities on redressing grievances of constituents, 'parliament men' who monitor the institutional structures ,and 'ministerial aspirants' who seek promotion to the government ranks (Searing 1994, 16; Searing 1991, 1253). While such roles may be chosen by legislators depending on situational contexts (Blomgren and Rozenberg 2012, 9), any of these, however, allow for considerable scope to translate intrinsic motives into actual legislative behaviour (Searing 1991, 1253). As Searing notes, ‘(e)motional incentives are the principal energizing forces in all parliamentary roles' (Searing 1994, 19). Plausibly, intrinsic motives are shaped to an extent by social group membership and the personal experiences legislators have had during their life, which may make them 'refer, consciously or unconsciously, to their biography when developing their preferences’ (Bäck and Debus 2016, 34; Bäck, Debus, and Müller 2014, 507).

With regard to immigration-related legislative behaviour, attention is often raised to legislators' own migratory backgrounds (Wüst 2014a; Saalfeld and Bischof 2013; Blätte and Wüst 2017; Saalfeld 2011). The assumption is that experiences of migration and the obstacles of integrating oneself into society create a special awareness of, and interest in, the challenges and problems of the 'Einwanderungsgesellschaft'. Especially immigrant-origin politicians with visible traits, such as foreign-sounding names and/or non-Caucasian appearance, may have been constantly reminded in their lives to be 'distinct' from the majority society, creating an intrinsic motivation or, in Searing words, 'emotional incentive' to focus their parliamentary activities on immigration-related issues. Indeed, a number of empirical studies, for example in Germany (Blätte and Wüst 2017; Wüst 2014a) and in the UK (Saalfeld and Bischof 2013; Saalfeld 2011), suggest that immigrant-origin legislators focus more strongly on migration-specific issues, especially those with a 'visible' background. 
Based on the reviewed theoretical and empirical literature, visible immigrantorigin legislators may be thus more intrinsically motivated to speak about the refugee and asylum issue than other legislators.

\section{Legislators' incentives to cultivate a personal vote and speech attention to immigration}

A second literature adheres to the logic of principal-agent theory, according to which legislators are assumed to act as agents who are electorally accountable to voters, thus responding to the demands of this principal, providing for a vertical representation link between ruled and rulers (Mitchell 2000; Carey 2009). Re-election is assumed to be the central motivation for legislators' responsiveness to voters, because re-election is a necessary precondition for any other career-related goal, whether policy influence or prestigious offices in parliament (Strøm 1997). Thus, if their re-election is uncertain, legislators may use their legislative repertoire to take positions, claim credit and/or advertise their personal qualities for the purpose of attracting personal votes (Mayhew 1974).

Aligning their own issue priorities with those of voters is intimately related to legislators' personal vote-seeking strategies. Indeed, commonly considered an important feature of (collective) party responsiveness (Budge and Farlie 1983; Spoon and Klüver 2014; Green-Pedersen and Otjes 2017), issue attention considerations should also rank high in legislators' individual strategies to cater to voters. Similarly to the argument that parties have vote-seeking incentives to align own issue priorities with that of voters (Spoon and Klüver 2014), legislators should seek to prioritise issues that are important to voters as an expression of catering to their political demands. Taking policy positions presumes that political actors dedicate a minimum of attention to the policy issue on which positions are taken (Budge and Farlie 1983). Why would a voter feel attracted to 
a legislator who takes a similar policy position on an issue but who does not convincingly show that $\mathrm{s} /$ he considers the issue important? Therefore, individual legislators seeking personal votes should see reasons to align their own issue priorities with that of their voters. If the refugee and asylum issue becomes more salient on the general political agenda and in the minds of voters, individual legislators may thus seek to increase attention to this issue in their speeches as well.

Commonly, legislators' personal vote-seeking incentives are said to be shaped by the way electoral rules transfer votes into seats (Carey and Shugart 1995; Carey 2009; Mitchell 2000). Only under electoral rules which provide voters the possibility to influence directly the electoral fate of individual legislators, for example under conditions of single-member plurality elections (SMD) rather than under conditions of closed-list proportional representation (PR), should legislative behaviour be tailored to appeal to local voters (Carey and Shugart 1995; Mitchell 2000). Conversely, under conditions of closed-list PR in districts of high magnitude, voters are typically provided with long lists of candidates, which they can only approve or defect as a whole. Here, legislators' electoral fate depends mainly on the list position allocated by their parties, such that they have fewer incentives to cultivate a personal vote (Carey and Shugart 1995; Carey 2009). Therefore, legislators should have stronger incentives to align their own issue priorities with that of voters under SMD, while closed-list PR should provide stronger incentives to be loyal to the party.

However, recent scholarship suggests that the effect of electoral rules is not universal in the sense that all legislators face the same personal vote-seeking incentives just because they are elected under the same set of electoral rules. These works take into account that the personal vote-seeking behaviour of legislators elected in SMDs are conditional on their individual levels of electoral vulnerability (Zittel and Gschwend 
2008; André, Depauw, and Martin 2015). When SMD legislators' districts are 'safe' seats, because they won it in the previous election with a comfortable vote margin, they may not be too concerned about reaching their re-election goals, attenuating their personal vote-seeking incentives. Conversely, if the previous constituency election was a close race, they should be more concerned about potential vote losses, which should create stronger incentives to align their own issue attention to that of voters.

\section{The constraining influence of parliamentary party groups on individual}

\section{speechmaking}

The two theoretical perspectives outlined so far share the assumption that legislators' individual motives are underlying their speech attention to the refugee and asylum issue. However, relying on this assumption disregards that PPGs are important contexts for legislative behaviour in general, and for speechmaking in particular.

As Cox (Cox 2006, 142-49) puts it, PPGs are essential to parliamentary decision-making, because they help solving the problem of a 'plenary bottleneck'. That is, without hierarchically organised PPGs controlling the plenary agenda and thus creating inequalities in legislators' access to the plenary, it would be impossible to coordinate which subset of all proposed bills can make it through the bottleneck, given the hard budget constraint on time and the fact that each individual member has motive to consume plenary time. As rulers over the parliamentary floor, PPG leaders may be thus conceptualised as utilising legislators as a resource to reach two types of party goals, that is, policy and votes (Müller 2000). Therefore, we may refer here again to the principal-agent conception, describing a vertical hierarchical link between PPG leaders and legislators, with the latter supposed to act upon the instructions of the former.

First, in order to reach their policy-seeking goals, PPG leaders must take into account that policy-making takes place in various (sub-) policy areas. Therefore, an 
internal division of labour is a necessary feature of PPGs, whereby legislators are supposed to serve their PPGs as experts in different (sub-) policy areas. Policyspecialised committee structures help PPGs ensuring such horizontal coordination (Saalfeld and Strøm 2014). Moreover, due to the tight vertical organisation of PPGs in most parliamentary democracies, vertical and horizontal coordination interact. That is, the vertical grip of PPG leaders over legislators reach down into their horizontally differentiated policy/committee specialisation (Damgaard 1995). Thus, PPG leaders should be more likely to delegate plenary time to legislators on topics that fall into the area of their policy specialisation.

Arguably, when choosing legislators to become policy experts in certain policy areas/committees, it should make sense for PPGs to take into account their personal skills and experiences. As immigrant-origin legislators have often distinct experiences of immigration and societal and cultural integration, they may be more likely to be perceived by PPG leaderships as experts on immigration. Moreover, if their immigrant background is visible, parties can utilise this trait for signalling expertise on the parliamentary floor to voters. This bias is also suggested by Nergiz' (2014) interviewbased qualitative research on immigrant-origin legislators in Germany. Assigning immigrant-origin legislators for expert positions on migration is supposed to create an impression of what Nergiz dubs 'authenticity'. As one interviewee puts is: '(i)t is important how well spokespersons can present the message of our party, regardless of whether the person has migratory experience or not. [...] However, when people see a migrant-origin person, then it is more convincing - people realise that I know what I am talking about' (Nergiz 2014, 255). Thus, an alternative explanation for the finding that legislators with a visible immigrant background are more likely to talk about migrantrelated issues in the plenary may be their ascribed policy expertise on that matter, 
serving the need for a distinct division of labour within PPGs. It follows that, if policy specialisation is the driving mechanism, immigrant-origin legislators with a visible background should talk more about the refugee and asylum issue than other legislators, conditional on their assignment to committees more likely to deal with matters of immigration and integration policy.

In addition to that, it can be argued that the impact of visible immigrant-origin legislators should be further conditional on the size of their PPGs. The intuition is that larger PPGs provide better conditions for an efficient division of labour. To the contrary, in smaller PPGs there is less personnel available to be assigned to an equally large number of (sub-)policy areas. This challenge is reflected in legislators' membership in policy-specialised committees. In Germany, for example, legislators from larger PPGs can normally concentrate on one committee membership, while legislators from smaller PPGs have to split their parliamentary work across several committees (Ismayr 2012, 168; Ismayr 1992, 189-91). Since legislators in smaller PPGs are supposed to develop an expertise in more areas than legislators in larger PPGs, legislators' individual areas of expertise are more diverse and are thus more blurred across legislators in smaller PPGs, while they are more distinct and delineated in larger PPGs. Accordingly, legislators from larger PPGs may speak overall about fewer issue areas on the parliamentary floor, while the lack of personnel in smaller PPGs makes it necessary that legislators have to speak not just about one, but about several issues, blurring the issue areas which they focus upon in their speeches altogether. Therefore, differences in speech attention to immigration between visible immigrant-origin and other legislators should be less pronounced in smaller than in larger PPGs.

Second, speeches provide parties a public platform to communicate their policy positions, to claim credit and to advertise the party label as an electoral strategy to 
attract voters (Bächtiger 2014). For this purpose, PPG leaders should value a unified party message, which they should seek to protect from deviant behaviour of legislators from within their own ranks (Proksch and Slapin 2015; Bäck and Debus 2018; Bäck and Debus 2016). Proksch and Slapin describe the potentially tense relationship between PPG leaders and legislators in a formal model of a delegation game (Proksch and Slapin 2015). The extent to which PPG leaders place importance on a unified party position depends in this model on the extent to which the electoral system provides for partybased voting cues. Where legislators' individual reputation is an important source for gaining seats, for example in the British SMD system, PPG leaderships are more at ease with legislators deviating from the party line in their speeches. To the contrary, in closed-list PR systems, where citizens cast votes for a party label rather than for individual candidates, PPGs should try to circumvent deviant voices from within their own ranks. Similarly, in political systems like the German mixed-member proportional (MMP) system, where party-based voting cues are of utmost importance to parties' electoral performance, PPG leaderships have strong incentives to maintain control over who speaks about what.

Therefore, under MMP rules, PPG leaderships should seek to deny legislator access to the parliamentary floor if these follow own personal vote-seeking strategies (see also Bäck and Debus 2018). Especially if debates are about salient issues that have the potential to damage the party label, PPG leaders should be wary of this type of legislator. For this reason, contrary to the expectation made in the previous subchapter, SMD legislators from marginal seats may speak less, and not more, about such issues in their speeches than SMD legislators from 'safe' seats. 


\section{Empirical context and hypotheses}

To examine this theoretical framework empirically, the present study focuses on the 18th German Bundestag for a number of reasons. First, the number of legislators of immigrant-origin has steadily grown over the past two decades, reaching an unprecedented high in the 18th Bundestag, in which 37 immigrant-origin legislators were elected, making up for 5.9\% of the entire parliament (Wüst 2014b).

Second, Germany employs an MMP system, which provides for within-country variation of electoral rules. There are 299 MPs elected in SMDs and a slightly larger number of MPs elected in 16 multi-member districts under closed-list PR. However, since mainly the two largest parties, the Christian and the Social Democrats, have realistic chances of winning SMD seats, almost all SMD legislators are affiliated with these two parties. The electoral system is further compensatory, such that parties' overall seat shares are determined by their vote shares in the PR tier (Saalfeld 2005). Therefore, while (government) legislators elected in SMDs should desire re-election in local constituencies, PPG leaderships should be mainly interested in maximising their PR votes (Proksch and Slapin 2015).

Third, the Bundestag is a paradigmatic case of a party-controlled Arbeitsparlament ('working parliament'), based on hierarchically organised PPGs cutting across a system of policy-specialised committees, ensuring a strongly partycontrolled division of labour among legislators (Miller and Stecker 2008). Since it is in the discretion of PPG leaderships to assign and withdraw legislators to committees, they decide implicitly which policy areas legislators specialise in (Miller and Stecker 2008; Damgaard 1995). As policy experts, legislators are supposed to represent the official position of the party, enforced by the thread of sanctions from the PPG leadership, which range from subtle persuasion and threats, to the withdrawal of the committee 
seat, to the ultimate denial of upward promotion within the hierarchy of the PPG (Miller and Stecker 2008; Ismayr 2012, 169). Similarly, PPG leaderships control access to the parliamentary floor in the Bundestag. Ismayr describes the process of speaker selection in the German Bundestag as being decided by chairpersons of PPGs' working groups who are also members of the PPG leadership (Ismayr, 2012, p. 305).

Fourth, the political context of the $18^{\text {th }}$ Bundestag was one of a serious immigration shock, which has turned the issue of refugees and asylum into a hot one of German politics. Since 2013, immigration to Germany has steadily increased until late 2015. Official numbers report an influx of overall 1.1, 1.3 and 2 million immigrants in 2013, 2014 and 2015 respectively. The peak in 2015 was fuelled by an estimated influx of 890,000 asylum seekers, mainly in the second half-year (Bundesamt für Migration und Flüchtlinge 2016). This immigration shock has also been a consequence of the federal government's decision to suspend the Dublin Agreement in 2015, a decision that opened the border for a high number of refugees, who were stuck in Budapest/Hungary hoping to continue their journey mainly from war-torn Syria to Germany and other Northern and Western European Countries.

(Figure 1 near here)

Given the huge logistic, administrative and, indeed, political challenges that the intake of such high numbers of immigrants caused, nationwide opinion polls testified soon that the issue of immigration, asylum and the integration of refugees became the 'most important political problem' for German citizens, as shown in Figure 1. The figure shows data from the Politbarometer, a monthly repeated opinion survey, which asks in open-ended questions what respondents consider the most important current problem (Forschungsgruppe Wahlen 2018). The graph relates to the percentage of respondents referring to immigration in their answers between 2000 and 2018. As seen, 
the percentage of respondents doing so increased steadily since 2013 to reach a high of almost $90 \%$ in the second half of 2015 . Taking advantage of the increased saliency of the refugee and asylum issue, a new right-wing populist party, the 'Alternative für Deutschland' (AfD), entered the electoral stage to capitalise on votes by the use of antiimmigrant rhetoric and position taking. Consequently, due to the high salience of the immigration issue the AfD posed an electoral threat to the established parties, in particular to the government parties responsible for immigration policy, the Social Democrats (SPD) and the two Christian Democratic sister parties (CDU/CSU).

Against the political and institutional context of the $18^{\text {th }}$ Bundestag, and based on the theoretical framework outlined in the theory section, two sets of competing hypotheses are derived.

First, the context of the sudden refugee crisis of the $18^{\text {th }}$ Bundestag provides for a critical test of a direct effect of intrinsic motives encoded in the visibility of immigrant-origin legislators on immigration-related speechmaking. Consequently, a first hypothesis reads that visible legislators of immigrant-origin were more likely to speak about the refugee and asylum issue than other legislators (H1). Alternatively, two competing hypotheses aim at testing whether these legislators talk more about the issue, because they are more likely to be selected as policy experts serving the need for an efficient division of labour within their PPGs rather than out of intrinsic motives. As policy expertise can be plausibly assumed to be reflected in legislators' committee memberships to a certain extent, visible immigrant-origin legislators should be more likely to speak about immigration issues as policy experts, if they sit on committees that are more likely to deal with immigration-related issues (for example the social affairs and interior committee rather than the environment or defence committee). Thus, a first competing hypothesis is that the positive effect of visible legislators of immigrant- 
minority origin on speech attention to the refugee and asylum issue is mainly driven by their assignment to immigration-related committees (H2.1).

Additionally, if policy expertise ascribed by the PPG leadership is the driving mechanism, the organisational capacities of the PPG should condition the extent to which visible immigrant-origin legislators have a stronger focus on immigration issues than other legislators. Since conditions for an efficient division of labour are better in larger than in smaller PPGs, legislators' policy specialisations tend to be more blurred in smaller PPGs, as described in the previous section. In the $18^{\text {th }}$ Bundestag, the CDU/CSU and the SPD provide for cases of larger PPGs (311 and 192 seats respectively), and the Greens and the Left for cases of smaller PPGs (63 and 64 seats respectively). This provides for a test of yet another hypothesis competing with $\mathrm{H} 1$ : legislators of immigrant-minority origin speak more about the refugee and asylum issue than other legislators, conditional on the size of their PPGs (H2.2).

The second set of competing hypotheses contrasts individual against collective electoral motives. On one hand, with the refugee and asylum issue dominating the political agenda, SMD legislators should have had incentives to raise attention to this issue in their speeches to align their own issue attention with that of voters. However, this incentive should become stronger as a function of their individual level of electoral vulnerability. A third hypothesis is thus that SMD legislators were more likely to speak about the refugee and asylum issue the higher their electoral vulnerability (H3).

On the other, as the refugee crisis posed a serious electoral threat to the two government parties (SPD and CDU/CSU), which were increasingly under siege against the general perception that the government was not able to handle the crisis, further fuelled by the tireless attacks of the AfD criticising the government's immigration policy, the PPG leaderships of the CDU/CSU and SPD should have had strong 
incentives to 'close their own ranks' in order to protect the party label from taking further damage. Thus, PPG leaders of the government parties should have been especially wary of providing access to debates on immigration to legislators who followed own electoral strategies in relation to the refugee and asylum issue. Consequently, a fourth hypothesis reads that SMD legislators were less likely to speak about the refugee and asylum issue in the $18^{\text {th }}$ Bundestag the higher their electoral vulnerability (H4).

\section{Data and methods}

In order to examine these hypotheses, this study relies on a dataset containing a corpus of all oral contributions in the plenary during the $18^{\text {th }}$ German Bundestag. The data is provided by OffenesParlament.de, an NGO website committed to making parliamentary processes transparent (OffenesParlament.de 2018). Additionally, a second dataset was collected containing legislator-level variables to be described in due course. Since both datasets contain information on the same legislators, it was possible to merge the two into one dataset.

\section{Using structural topic models to measure speech attention to immigration}

In order to measure speech attention to the issue of immigration, this paper relies on a recently developed automated method for textual analysis: structural topic models (STM) (Roberts et al. 2014). STM belongs to the family of unsupervised 'topic models', which rely on clustering algorithms to code a collection of texts (a corpus) into several topics based on patterns of word co-occurrence in the texts (Grimmer and Stewart 2013). For the purpose of this paper, the assumption is that these topics can be interpreted as latent issue attention of speeches. For technical details, a description of 
the text pre-processing as well as additional validation analyses, please see section A1 in the online appendix.

Based on an STM with $\mathrm{k}=13$ topics, Table 1 gives an overview over the six most discriminating terms in each topic, so-called FREX terms, that is, words which are highly likely to appear in a topic (frequent) while also being less likely to appear in other topics (exclusive) (Roberts et al. 2014). The first topic clearly identifies speech attention to immigration with a strong bias towards refugee and asylum issues, indicated by the most discriminating words 'flüchtlingen' (refugees), 'flüchtling' (refugee), 'asylbewerb' (asylum seeker), 'integr' (integration), 'asyl' (asylum) and 'migrat' (migration).

(Table 1 near here)

Although it cannot completely be ruled out that other aspects of immigration politics may be covered to some extent in the topic, validation analyses presented in the appendix section A1 provide evidence that the found immigration topic corresponds mainly to the refugee and asylum issue. This interpretation is further substantiated by an assessment of what Grimmer and Stewart dub the 'predictive validity' of a topic, that is, an assessment of how well variation in topic usage corresponds over time with expected events (Grimmer and Stewart 2013). For this purpose, Figure 2 visualises how the temporal development of the immigration topic in the $18^{\text {th }}$ Bundestag (2013-2017) corresponds to the issue attention of citizens expressed in the 'most important problem' question of the monthly waves of the Politbarometer survey (Forschungsgruppe Wahlen 2018), already utilised in Figure 1. As seen, not only peaked citizens' issue attention at the climax of the European refugee crisis in the second half of 2015, but also the found topic as measured by the $\mathrm{k}=13 \mathrm{STM}$ (with $95 \%$ confidence intervals).

(Figure 2 near here) 


\section{Dataset}

While the dependent variable, speech attention to the refugee and asylum issue, is measured at the level of 10,379 speeches utilising the $k=13$ STM, a number of legislator-specific variables extend the dataset further. Descriptive statistics of all variables are shown in section A2 of the appendix file.

Main independent variables include the following. Visible immigrant-origin legislator takes a value of one if a speech is given by such a legislator, and zero otherwise. This group of legislators is defined and coded according to two criterions. First, the criterion immigrant-origin applies to all legislators born either (a) abroad or in Germany with foreign nationality at birth, or (b) born with German nationality and at least one parent of foreign nationality at birth. In coding practice, however, Wüst's (2014b) list of 37 immigrant-origin legislators was taken as a point for departure. Second these legislators were coded based on whether or not they have visible traits that make them objectively identifiable as having an immigrant background. Although defining oneself as belonging to an immigrant minority group is essentially a question of subjective self-perception, in practice, however, it is often unfeasible for social researchers to capture this subjectivity (Blätte and Wüst 2017, 210-11; Heath et al. 2013, 15-17). For this reason, scholars commonly rely on objective concepts, such as the Canadian definition of visible minorities, which can be applied to immigrant-origin legislators who are identifiable as 'non-Caucasian or non-white' (Wüst 2014a, 102; Heath et al. 2013, 17). However, since the present paper has a central interest in the immigration expertise and/or signalling purpose of immigrant-origin legislators ascribed by PPG leaderships (i.e. the 'authenticity' of the spokesperson [Nergiz 2014, 254]), visibility refers here also to immigrant backgrounds that can be objectively inferred from the name of the speaker. 
Moreover, a set of dummy variables distinguishes speakers' PPG affiliations. Migration-related committee is a dichotomous variable measuring whether speech givers sit on committees more likely to deal with immigration based on a modified categorisation scheme proposed by Wüst (Wüst 2011) ${ }^{1}$. Election mode distinguishes whether a speaker was elected from a party list $(=0)$ or in an SMD $(=1)$. In addition, vote margin measures the difference in votes between the SMD winner and the second best loser in the SMD race, in which the speaker was running as a candidate in the 2013 Bundestag election. Since most list legislators ran as double candidates in party lists and in SMDs simultaneously, this variable takes also values for most list legislators except those who did not run in an SMD race simultaneously.

A set of control variables is supposed to account for other variation influencing speech attention to immigration. To account for the dynamic evolution of the refugee and asylum issue over time, days till election measures the date of a speech in days until the 2017 election. By including also the squared term of days till election, it is intended to better control for non-linear effects of time. Megaseat distinguishes backbencher legislators from those who occupy a parliamentary position of influence, defined as committee chairs and members of the PPG leaderships including the chairpersons of working groups. Seniority approximates the career stage by counting the years legislators have been serving in the Bundestag prior to the 2013 election. Female takes values of one for female legislators and zero otherwise. Moreover, key sociodemographic features at the SMD level are accounted for, with the local foreigner share, the population share of residents older than 60 and the unemployment rate. And finally, East is a dichotomous variable distinguishing legislators elected in the territory of the former GDR, to account for the fact that the Eastern German population was, on average, more critical of the governments' immigration policy than the rest of the 
German population, indicated by a higher support for the AfD and frequent antiimmigrant protests (PEGIDA).

\section{Analysing speech attention to immigration in the 18th Bundestag}

Table 3 presents the results of fractional response logit models estimating speech attention to immigration in the $18^{\text {th }}$ Bundestag. Fractional response models are appropriate here because the dependent variable is bound to the zero-one interval. Under such conditions, standard OLS regression models cannot guarantee that the predicted values of the dependent variable are restricted to the unit interval and would thus yield biased predictions for extreme values of the independent variables (Papke and Wooldridge 1996). ${ }^{2}$ The regression models take speeches as the units of analysis, estimating robust standard errors clustered at the level of legislators to account for the fact that speeches delivered by the same legislator are interdependent.

(Table 3 near here)

Models 1 to 3 are supposed to evaluate the first set of competing hypotheses, that is, whether visible immigrant-origin legislators are universally more likely to speak about immigration, or whether this effect is conditional on their committee specialisation and the size of their PPGs. While Model 1 estimates the effects of the independent variables without any interactions, Model 2 includes an interaction between these legislators and migration-related committee. Model 3 includes the interaction with the party variable.

(Figure 3 near here)

Model 1 suggests a positive and statistically significant effect of visible immigrant-origin legislators. However, Models 2 and 3 suggest that this effect is strongly dependent on legislators' committee specialisation and on PPG size. Figure 3 
visualises these findings in the form of predicted immigration topic proportions with 95\% confidence intervals. The left-hand panel is based on Model 2 and shows that both types of legislators are significantly more likely to speak about immigration when they sit on migration-related committees. Although visible immigrant-origin legislators who sit on migration-related committees are estimated to talk slightly more about immigration than other legislators who also sit on such committees, this difference is not statistical significant. This is, in line with hypothesis 2.1 , first evidence that legislators' policy specialisation drives speech attention to immigration more strongly than immigrant-origin legislators' intrinsic motives.

The right-hand panel of Figure 3 corroborates this interpretation with additional empirical evidence. Based on the interaction of Model 3, the figure shows how speech attention to immigration varies depending on the visible immigrant-origin status of legislators and the size of their PPGs. As seen, in the two larger PPGs (CDU/CSU and SPD), which should allow for a more efficient division of labour, visible immigrantorigin legislators are estimated to talk significantly more about immigration than their PPG colleagues do. By contrast, the difference between the two types of legislators in the two smaller PPGs (Greens and The Left) is not only substantially negligible, but the wide overlap of confidence intervals suggests that their estimated speech attention to immigration is also statistically indistinguishable, as suspected by hypothesis 2.2 . Taken together, these results support hypotheses 2.1 and 2.2 rather than hypothesis 1 .

Models 4 and 5 engage with the second set of competing hypotheses, which dispute over whether SMD legislators should talk more or less about immigration as their individual level of electoral vulnerability increases. Model 4 and 5 extend Model 1 by variables measured at the SMD level. As this kind of information can only be assigned to legislators who ran as SMD candidates in the 2013 election, missing values 
apply to 479 speeches delivered by PR legislators who did not run as double candidates. In order to evaluate whether and how this effect is contingent on legislators' election mode, three groups of legislators are distinguished in Models 4 and 5, that is, government SMD legislators, consisting of government legislators elected in SMDs, government PR legislators, consisting of government legislators elected in party lists, and opposition legislators. Differences in election modes among opposition legislators are not considered, because it is mainly the two larger parties that won SMD races in the 2013 election. In Model 5, this variable is interacted with the local vote margin. Figure 4 visualises the conditionality of the vote margin effect on legislators' election mode. As can be seen, government legislators elected in SMDs tend to speak less about immigration as their local electoral vulnerability increases (higher vote margins indicate lower electoral vulnerability). To the contrary, there is no relationship whatsoever between speech attention to immigration and the local vote margin for list legislators, regardless of whether these belong to the government or to the opposition. This evidence favours hypothesis 4 over hypothesis 3.

(Figure 4 near here)

\section{Conclusion}

This paper was motivated by an interest in understanding speech attention to immigration in a party-constrained parliament at times when the issue dominates the political agenda. Two literatures suggest that legislators' individual motives may be underlying legislators' speech attention to immigration. On one hand, immigrantminority legislators may speak more about immigration than other legislators, due to intrinsic motives. On the other, legislators may speak more about immigration out of extrinsic motives to cultivate a personal vote with local constituents. However, a more 
realistic approach takes into account that PPG leaderships control speechmaking. Thus, the effects of variables measuring legislators' individual motives, here their immigrantminority origin and personal vote-seeking incentives, should be conditional on the organisational capacities and gatekeeping role of PPG leaderships. Based on an STM analysis of more than 10,000 speeches held in the $18^{\text {th }}$ Bundestag, a legislative period in which Germany was hit by the refugee crisis, results indicate that legislator' speech attention to immigration is more strongly shaped by PPG specific factors than by their individual motives.

Thus, this paper bridges and contributes to the literatures on the presence of immigrant-origin legislators, issue attention to immigration and parliamentary speechmaking. First, while a link between immigrant-origin legislators with a visible background and migration-specific speechmaking has been previously reported for Germany (Blätte and Wüst 2017), the present paper proposes to outline the limits of this link. Findings suggest that in the Bundestag, a prime example of a party-controlled parliament, the effect of visible legislators of immigrant-origin on speech attention to immigration is contingent on the level of labour division within PPGs. These legislators seem to focus on the refugee and asylum issue in their speeches due to their role as policy experts, serving the need for an efficient division of labour within PPGs, rather than out of intrinsic motives. This interpretation is also supported by the interviewbased research of Nergiz $(2014,253-263)$. Several interviewees explained that they took an expert position on immigration and integration within their parties out of careerrelated motives or because they were imposed to do so, rather than out of own interest. . Similarly, Wüst and Saalfelds' comparative research in Germany, France, Sweden and the UK suggests that immigrant-origin legislators' behaviour is shaped by the parliamentary opportunity structure rather than caused by their personal ethnic origin or 
migration experiences (Wüst and Saalfeld 2010, 331). The finding of the present paper complements these works by suggesting that ascribed policy expertise, rather than intrinsic motives, shapes a concrete legislative behaviour at the heart of parliamentary life: legislative speechmaking.

Second, the present study makes a contribution as the results indicate that legislators' incentives to cultivate a personal vote may shape their speech attention to immigration in a context in which the immigration issue is highly salient. Drawing on the burgeoning literature on parliamentary speechmaking (Proksch and Slapin 2015; Bäck, Debus, and Müller 2014; Bäck and Debus 2018; Bäck and Debus 2016), it is shown that in such a context government legislators elected in SMDs talk less about immigration as their individual level of electoral vulnerability increases. Refugees and asylum was clearly one of the most salient issues during the $18^{\text {th }}$ Bundestag term. In this context, the two PPGs in government (CDU/CSU and SPD) are likely to have incentives to circumvent criticism on the government's immigration policy from within the party in order to maintain a unified party position. Therefore, it may have been a strategic decision to prevent legislators from talking about immigration if these had own personal vote-seeking incentives to disobey the party line to improve individual electoral prospects.

This study calls for future research in at least two respects. First, while this paper had a focus on speech attention, future research may shed more light on the specific policy positions that legislators communicate in their speeches. These may be inferred directly from legislators' speeches by the use of scaling methods of textual analysis (e.g. Bäck and Debus 2016, 48-74).

Second, a natural extension would be a comparative study in order to examine how the moderating influence of PPG contexts can differ depending on the level of 
party control. Future research should increase variation with regard to how strongly PPGs control floor access and legislators' policy specialisations. Legislative debates in more legislator-centred parliaments, like the UK House of Commons or the US House of Representatives, may provide legislators a more open stage to express their individual immigration-related concerns. As a corollary, while dissenting voices may remain widely unheard in party-centred parliaments like the Bundestag, legislatorcentred parliaments may produce more polarisation in legislative debates on immigration, especially at times when the issue dominates the political agenda. 


\section{References}

André, Audrey, Sam Depauw, and Shane Martin. 2015. "Electoral Systems and Legislators' Constituency Effort: The Mediating Effect of Electoral Vulnerability." Comparative Political Studies 48 (4): 464-96.

Bächtiger, André. 2014. "Debate and Deliberation in Legislatures.” In The Oxford Handbook of Legislative Studies, edited by Kaare Strøm, Thomas Saalfeld, and Shane Martin, 145-66. Oxford.

Bäck, Hanna, and Marc Debus. 2016. Political Parties, Parliaments and Legislative Speechmaking. Basingstoke: Palgrave Macmillan.

- 2018. "Representing the Region on the Floor: Socioeconomic Characteristics of Electoral Districts and Legislative Speechmaking." Parliamentary Affairs 71 (73-102).

Bäck, Hanna, Marc Debus, and Jochen Müller. 2014. "Who Takes the Parliamentary Floor? The Role of Gender in Speech-Making in the Swedish Riksdag." Political Research Quarterly 67 (3): 504-18.

Blätte, Andreas, and Andreas M. Wüst. 2017. “Der migrationsspezifische Einfluss auf parlamentarisches Handeln: Ein Hypothesentest auf der Grundlage von Redebeiträgen der Abgeordneten des Deutschen Bundestags 1996-2013.” Politische Vierteljahresschrift 58 (2): 205-33.

Blomgren, Magnus, and Olivier Rozenberg. 2012. "Legislative Roles and Legislative Studies: The Neo-Institutionalist Turning Point?” In Parliamentary Roles in Modern Legislatures, edited by Magnus Blomgren and Olivier Rozenberg, 8-36. London and New York: Routledge.

Budge, Ian, and Dennis J Farlie. 1983. Explaining and Predicting Elections: Issue 
Effects and Party Strategies in Twenty-Three Democracies. London: George Allen \& Unwin.

Bundesamt für Migration und Flüchtlinge. 2016. "Migrationsbericht 2015 - Zentrale Ergebnisse."

http://www.bamf.de/SharedDocs/Anlagen/DE/Publikationen/Migrationsberichte/m igrationsbericht-2015-zentrale-ergebnisse.pdf?_blob=publicationFile.

Carey, John M. 2009. Legislative Voting and Accountability. Cambridge: Cambridge University Press.

Carey, John M., and Matthew Soberg Shugart. 1995. "Incentives to Cultivate a Personal Vote: A Rank Ordering of Electoral Formulas.” Electoral Studies 14 (4): 417-39.

Cox, Gary W. 2006. "The Organization of Democratic Legislatures.” In The Oxford Handbook of Political Economy, edited by Barry R. Weingast and Donald A. Wittman, 141-61. Oxford: Oxford University Press.

Damgaard, Erik. 1995. "How Parties Control Committee Members.” In Parliaments and Majority Rule in Western Europe, edited by Herbert Döring, 308-24. Frankfurt am Main: Campus Verlag.

Forschungsgruppe Wahlen. 2018. "Politbarometer Wichtige Probleme 2000-2018." http://www.forschungsgruppe.de/Umfragen/Politbarometer/Langzeitentwicklung__Themen_im_Ueberblick/Politik_II/9_Probleme_1_1.xlsx.

Goerres, Achim, Sabrina J. Mayer, and Dennis C. Spies. 2018. "Immigrant Voters against Their Will: A Focus Group Analysis of Identities, Political Issues and Party Allegiances among German Resettlers during the 2017 Bundestag Election Campaign." Journal of Ethnic and Migration Studies online fir. doi:10.1080/1369183X.2018.1503527.

Green-Pedersen, Christoffer, and Simon Otjes. 2017. "A Hot Topic? Immigration on the 
Agenda in Western Europe.” Party Politics. doi:10.1177/1354068817728211.

Grimmer, Justin, and Brandon M. Stewart. 2013. “Text as Data: The Promise and Pitfalls of Automatic Content Analysis Methods for Political Texts.” Political Analysis $1(1-31)$.

Heath, Anthony F., Stephen D. Fisher, G. Rosenblatt, David Sanders, and Maria Sobolewska. 2013. The Political Integration of Ethnic Minorities in Britain. Oxford: Oxford University Press.

Ismayr, Wolfgang. 1992. Der Deutsche Bundestag. Funktionen - Willensbildung Reformansätze. Opladen: Leske and Budrich.

- 2012. Der Deutsche Bundestag. Wiesbaden: VS Verlag für Sozialwissenschaften.

Kortmann, Matthias, and Christian Stecker. 2017. "Party Competition and Immigration and Integration Policies: A Comparative Analysis." Comparative European Politics. doi:10.1057/s41295-017-0108-8.

Mayhew, David R. 1974. Congress: The Electoral Connection. New Haven and London: Yale University Press.

Miller, Bernhard, and Christian Stecker. 2008. "Consensus by Default? Interaction of Government and Opposition Parties in the Committees of the German Bundestag." German Politics 17 (3): 305-22.

Mitchell, Paul. 2000. "Voters and Their Representatives: Electoral Institutions and Delegation in Parliamentary Democracies.” European Journal of Political Research 37: 335-51.

Müller, Wolfgang C. 2000. "Political Parties in Parliamentary Democracies: Making Delegation and Accountability Work.” European Journal of Political Research 37 (3): 309-33. 
Nergiz, Devrimsel Deniz. 2014. I Long for Normality: A Study on German

Parliamentarians with Migration Backgrounds. Wiesbaden: Springer.

OffenesParlament.de. 2018. "Daten.” https://offenesparlament.de/daten/.

Papke, Leslie E., and Jeffrey M. Wooldridge. 1996. "Econometric Methods for Fractional Response Variables with an Application to 401(K) Plan Participation Rated." Journal of Applied Econometrics 11 (6): 619-32.

Proksch, Sven-Oliver, and Jonathan Slapin. 2015. The Politics of Parliamentary Debate. Cambridge: Cambridge University Press.

Roberts, Margaret E., Brandon M. Stewart, Dustin Tingley, Christopher Lucas, Jetson Leder-Luis, Shana Kushner Gadarian, Bethany Albertson, and David G. Rand. 2014. "Structural Topic Models for Open-Ended Survey Responses.” American Journal of Political Science 58 (4): 1064-82.

Ruedin, Didier, and Laura Morales. 2017. "Estimating Party Positions on Immigration." Party Politics, no. Online first. doi:10.1177/1354068817713122.

Saalfeld, Thomas. 2005. "Germany: Stability and Strategy in a Mixed-Member Proportional System." In The Politics of Electoral Systems, edited by Gallagher Michael and Paul Mitchell, 209-28. Oxford and New York: Oxford University Press.

_. 2011. "Parliamentary Questions as Instruments of Substantive Representation: Visible Minorities in the UK House of Commons, 2005-10." The Journal of Legislative Studies 17 (3): 271-89.

Saalfeld, Thomas, and Daniel Bischof. 2013. "Minority-Ethnic MPs and the Substantive Representation of Minority Interests in the House of Commons, 2005-2011.” Parliamentary Affairs 66 (2): 305-28.

Saalfeld, Thomas, and Kaare W. Strøm. 2014. "Political Parties and Legislators." In 
Oxford Handbook of Legislative Studies, 371-98. Oxford: Oxford University Press.

Searing, Donald D. 1991. "Roles, Rules, and Rationality in the New Institutionalism." American Political Science Review 85 (4): 1239-60.

— 1994. Westminster's World. Understanding Political Roles. Cambridge, MA: Harvard University Press.

Spoon, J.-J., and Heike Klüver. 2014. "Do Parties Respond? How Electoral Context Influences Party Responsiveness.” Electoral Studies 35: 48-60.

Strøm, Kaare. 1997. "Rules, Reasons and Routines: Legislative Roles in Parliamentary Democracies." In Members of Parliament in Western Europe: Roles and Behaviour, edited by Wolfgang C. Müller and Thomas Saalfeld, 155-74. London: Frank Cass.

Wüst, Andreas M. 2011. "Migrants as Parliamentary Actors in Germany.” In The Political Representation of Immigrants and Minorities. Voters, Parties and Parliaments in Liberal Democracies, edited by Karen Bird, Thomas Saalfeld, and Andreas M. Wüst, 250-65.

—. 2014a. "A Lasting Impact? On the Legislative Activities of Immigrant-Origin Parliamentarians in Germany." Journal of Legislative Studies, no. May: 37-41. doi:10.1080/13572334.2014.907601.

_. 2014b. "Immigration into Politics: Immigrant-Origin Candidates and Their Success in the 2013 Bundestag Election." German Politics \& Society 32 (3): 1-15. doi:10.3167/gps.2014.320301.

Wüst, Andreas M., and Thomas Saalfeld. 2010. “Abgeordnete Mit Migrationshintergrund Im Vereinigten Königreich, Frankreich, Deutschland Und Schweden: Opportunitäten Und Politikschwerpunkte.” Politische 
Vierteljahresschrift, Sonderheft 44. VS-Verlag: 312-33.

Zittel, Thomas, and Thomas Gschwend. 2008. "Individualised Constituency Campaigns in Mixed-Member Electoral Systems: Candidates in the 2005 German Elections.” West European Politics 31 (5): 978-1003.

Word count (including tables, references and endnotes): 8,460 
Notes

1 Migration-related committees are labour and social affairs; education and research; family, elderly and women; domestic affairs; culture and media; human rights; foreign affairs; European Union affairs; economic development aid; petitions.

2 The main results remain when using OLS with clustered standard errors or a multilevel random intercepts model. 
Tables and Figures 
Table 1: FREX terms and topic labels of STM with 13 topics.

\begin{tabular}{|c|c|c|}
\hline Topic no. & FREX terms & Topic label \\
\hline 1 & flüchtlingen, flüchtling, asylbewerb, integr, asyl, migrat & $\begin{array}{l}\text { Refugees and } \\
\text { Asylum }\end{array}$ \\
\hline 2 & bundeswehr, soldaten, soldatinnen, mali, mandat, afghanistan & Defense \\
\hline 3 & bahn, dobrindt, fahren, link, schien, auto & $\begin{array}{l}\text { Transportation } \\
\text { and } \\
\text { Infrastructure }\end{array}$ \\
\hline 4 & eltern, kind, jugendlichen, kindern, kinder, jugendlich & Family \\
\hline 5 & russland, türkei, ukrain, erdogan, saudi-arabien, demokrati & Foreign affairs \\
\hline 6 & $\begin{array}{l}\text { somalia, afrika, humanitär, entwicklungszusammenarbeit, } \\
\text { menschenrecht, entwicklungspolitik }\end{array}$ & $\begin{array}{l}\text { Development } \\
\text { aid }\end{array}$ \\
\hline 7 & $\begin{array}{l}\text { hochschulen, haushalt, bafög, bundeshaushalt, wohnungen, } \\
\text { mietpreisbrems }\end{array}$ & $\begin{array}{l}\text { Housing and } \\
\text { construction }\end{array}$ \\
\hline 8 & $\begin{array}{l}\text { rent, mindestlohn, rentenversicherung, arbeitnehm, männern, } \\
\text { arbeitnehmerinnen }\end{array}$ & Social \\
\hline 9 & $\begin{array}{l}\text { energien, energiewend, erneuerbaren, banken, klimaschutz, } \\
\text { ceta }\end{array}$ & Energy \\
\hline 10 & $\begin{array}{l}\text { patienten, pflege, arzt, versicherten, pflegeversicherung, } \\
\text { versorgung }\end{array}$ & Health \\
\hline 11 & $\begin{array}{l}\text { landwirtschaft, sport, digital, digitalisierung, ländlichen, } \\
\text { produkt }\end{array}$ & $\begin{array}{l}\text { Development of } \\
\text { rural areas }\end{array}$ \\
\hline 12 & $\begin{array}{l}\text { daten, untersuchungsausschuss, gericht, informationen, } \\
\text { täter, gesetzgeb }\end{array}$ & Justice \\
\hline 13 & parlament, argument, ausschuss, ehe, fraktionen, bisschen & Pro \\
\hline
\end{tabular}


Table 2: Determinants of speech attention to refugees and asylum

\begin{tabular}{|c|c|c|c|c|c|}
\hline & $\begin{array}{c}\text { Model } 1 \\
\mathrm{~b} / \mathrm{se}\end{array}$ & $\begin{array}{c}\text { Model } 2 \\
\text { b/se }\end{array}$ & $\begin{array}{c}\text { Model } 3 \\
\mathrm{~b} / \mathrm{se}\end{array}$ & $\begin{array}{c}\text { Model } 4 \\
\text { b/se }\end{array}$ & $\begin{array}{c}\text { Model } 5 \\
\text { b/se }\end{array}$ \\
\hline \multicolumn{6}{|l|}{ Independent variables: } \\
\hline PPG: SPD ${ }^{\text {a }}$ & $\begin{array}{l}-0.21 \\
(0.17)\end{array}$ & $\begin{array}{l}-0.21 \\
(0.17)\end{array}$ & $\begin{array}{l}-0.27 \\
(0.17)\end{array}$ & & \\
\hline PPG: Greens ${ }^{\mathrm{a}}$ & $\begin{array}{l}-0.36 \\
(0.24)\end{array}$ & $\begin{array}{l}-0.36 \\
(0.24)\end{array}$ & $\begin{array}{l}-0.25 \\
(0.25)\end{array}$ & & \\
\hline PPG: The Left ${ }^{\text {a }}$ & $\begin{array}{l}-0.46 \\
(0.28)\end{array}$ & $\begin{array}{l}-0.45 \\
(0.28)\end{array}$ & $\begin{array}{l}-0.38 \\
(0.29)\end{array}$ & & \\
\hline Visible immigrant-origin legislator & $\begin{array}{l}0.61^{*} \\
(0.25)\end{array}$ & $\begin{array}{l}-0.06 \\
(0.18)\end{array}$ & $\begin{array}{c}1.13 * * * \\
(0.24)\end{array}$ & $\begin{array}{c}0.53 \\
(0.29)\end{array}$ & $\begin{array}{l}0.58^{*} \\
(0.28)\end{array}$ \\
\hline Migration-related committee & $\begin{array}{c}0.59 * * * \\
(0.12)\end{array}$ & $\begin{array}{c}0.56^{* * *} \\
(0.12)\end{array}$ & $\begin{array}{l}0.59 * * * \\
(0.12)\end{array}$ & $\begin{array}{c}0.65 * * * \\
(0.13)\end{array}$ & $\begin{array}{c}0.66 * * * \\
(0.13)\end{array}$ \\
\hline $\begin{array}{l}\text { Visible immigrant-origin legislator } \\
* \text { migration-related committee }\end{array}$ & & $\begin{array}{l}0.77 * * \\
(0.28)\end{array}$ & & & \\
\hline $\begin{array}{l}\text { Visible immigrant-origin legislator } \\
\text { * SPD }\end{array}$ & & & $\begin{array}{c}0.25 \\
(0.46)\end{array}$ & & \\
\hline $\begin{array}{l}\text { Visible immigrant-origin legislator } \\
* \text { Greens }\end{array}$ & & & $\begin{array}{c}-0.99 * * * \\
(0.30)\end{array}$ & & \\
\hline $\begin{array}{l}\text { Visible immigrant-origin legislator } \\
* \text { The Left }\end{array}$ & & & $\begin{array}{l}-0.87^{*} \\
(0.39)\end{array}$ & & \\
\hline Vote margin & & & & $\begin{array}{l}1.71^{*} \\
(0.83)\end{array}$ & $\begin{array}{l}3.58^{* *} \\
(1.17)\end{array}$ \\
\hline Government PR legislator ${ }^{b}$ & & & & $\begin{array}{l}-0.03 \\
(0.19)\end{array}$ & $\begin{array}{l}0.47 \\
(0.24)\end{array}$ \\
\hline Opposition legislator $^{\mathrm{b}}$ & & & & $\begin{array}{l}-0.25 \\
(0.19)\end{array}$ & $\begin{array}{c}0.23 \\
(0.25)\end{array}$ \\
\hline $\begin{array}{l}\text { Government PR legislator * } \\
\text { vote margin }\end{array}$ & & & & & $\begin{array}{l}-3.24 * \\
(1.42)\end{array}$ \\
\hline $\begin{array}{l}\text { Opposition legislator * } \\
\text { vote margin }\end{array}$ & & & & & $\begin{array}{l}-3.51^{* *} \\
(1.33)\end{array}$ \\
\hline \multicolumn{6}{|l|}{ Speech/legislator-level controls: } \\
\hline Days_till_election & $\begin{array}{c}0.00 * * * \\
(0.00)\end{array}$ & $\begin{array}{c}0.00 * * * \\
(0.00)\end{array}$ & $\begin{array}{c}0.00 * * * \\
(0.00)\end{array}$ & $\begin{array}{c}0.00 * * * \\
(0.00)\end{array}$ & $\begin{array}{c}0.00 * * * \\
(0.00)\end{array}$ \\
\hline Days_till_election ${ }^{2}$ & $\begin{array}{c}-0.00 * * * \\
(0.00)\end{array}$ & $\begin{array}{c}-0.00 * * * \\
(0.00)\end{array}$ & $\begin{array}{c}-0.00 * * * \\
(0.00)\end{array}$ & $\begin{array}{c}-0.00 * * * \\
(0.00)\end{array}$ & $\begin{array}{c}-0.00 * * * \\
(0.00)\end{array}$ \\
\hline Megaseat & $\begin{array}{l}0.15 \\
(0.15)\end{array}$ & $\begin{array}{l}0.15 \\
(0.14)\end{array}$ & $\begin{array}{c}0.13 \\
(0.14)\end{array}$ & $\begin{array}{c}0.17 \\
(0.14)\end{array}$ & $\begin{array}{c}0.22 \\
(0.13)\end{array}$ \\
\hline Seniority & $\begin{array}{c}0.01 \\
(0.01)\end{array}$ & $\begin{array}{c}0.01 \\
(0.01)\end{array}$ & $\begin{array}{c}0.02 \\
(0.01)\end{array}$ & $\begin{array}{c}0.01 \\
(0.01)\end{array}$ & $\begin{array}{c}0.01 \\
(0.01)\end{array}$ \\
\hline Female & $\begin{array}{c}0.32 \\
(0.16)\end{array}$ & $\begin{array}{l}0.32 * \\
(0.16)\end{array}$ & $\begin{array}{l}0.34 * \\
(0.16)\end{array}$ & $\begin{array}{c}0.24 \\
(0.17)\end{array}$ & $\begin{array}{l}0.28 \\
(0.17)\end{array}$ \\
\hline Election mode: SMD & $\begin{array}{l}-0.07 \\
(0.19)\end{array}$ & $\begin{array}{l}-0.06 \\
(0.19)\end{array}$ & $\begin{array}{l}-0.04 \\
(0.19)\end{array}$ & & \\
\hline East & $\begin{array}{l}-0.08 \\
(0.19)\end{array}$ & $\begin{array}{l}-0.07 \\
(0.19)\end{array}$ & $\begin{array}{l}-0.11 \\
(0.19)\end{array}$ & $\begin{array}{l}-0.21 \\
(0.23)\end{array}$ & $\begin{array}{l}-0.22 \\
(0.23)\end{array}$ \\
\hline \multicolumn{6}{|l|}{ District-level controls: } \\
\hline Foreigner share in constituency & & & & $\begin{array}{l}-0.72 \\
(1.30)\end{array}$ & $\begin{array}{l}-0.68 \\
(1.34)\end{array}$ \\
\hline $\begin{array}{l}\text { Population share older than } 60 \\
\text { in constituency }\end{array}$ & & & & $\begin{array}{l}-0.77 \\
(2.99)\end{array}$ & $\begin{array}{l}-1.15 \\
(2.93)\end{array}$ \\
\hline
\end{tabular}


Unemployment rate in

$3.72 \quad 4.63$

constituency

(3.34)

(3.44)

INTERCEPT

$-4.27 * * *$

$-4.25 * * *$

$-4.28 * * *$

$-4.60 * * *$

$-4.92 * * *$

$\mathrm{N}$

(0.21)

(0.21)

(0.21)

$(0.90)$

(0.89)

$\mathrm{N}$

10379

10379

10379

9900

9900

Log pseudolikelihood

$\begin{array}{rrr}-1799.539 & -1798.464 & -1856.194 \\ 3625.077 & 3624.929 & 3619.489\end{array}$

$-1674.074$

$-1667.686$

AIC

3625.077

3378.148

3369.372

Note: Fractional response logit regression models: Table entries show unstandardised coefficients with robust

standard errors, clustered on legislators, in parentheses; ${ }^{\text {a }}$ reference category is CDU/CSU; ${ }^{b}$ reference category is

Government SMD legislator; $* \mathrm{p}<0.05, * * \mathrm{p}<0.01, * * * \mathrm{p}<0.001$ 
Figure 1. Percentage stating immigration and integration is most important problem 2000-2018

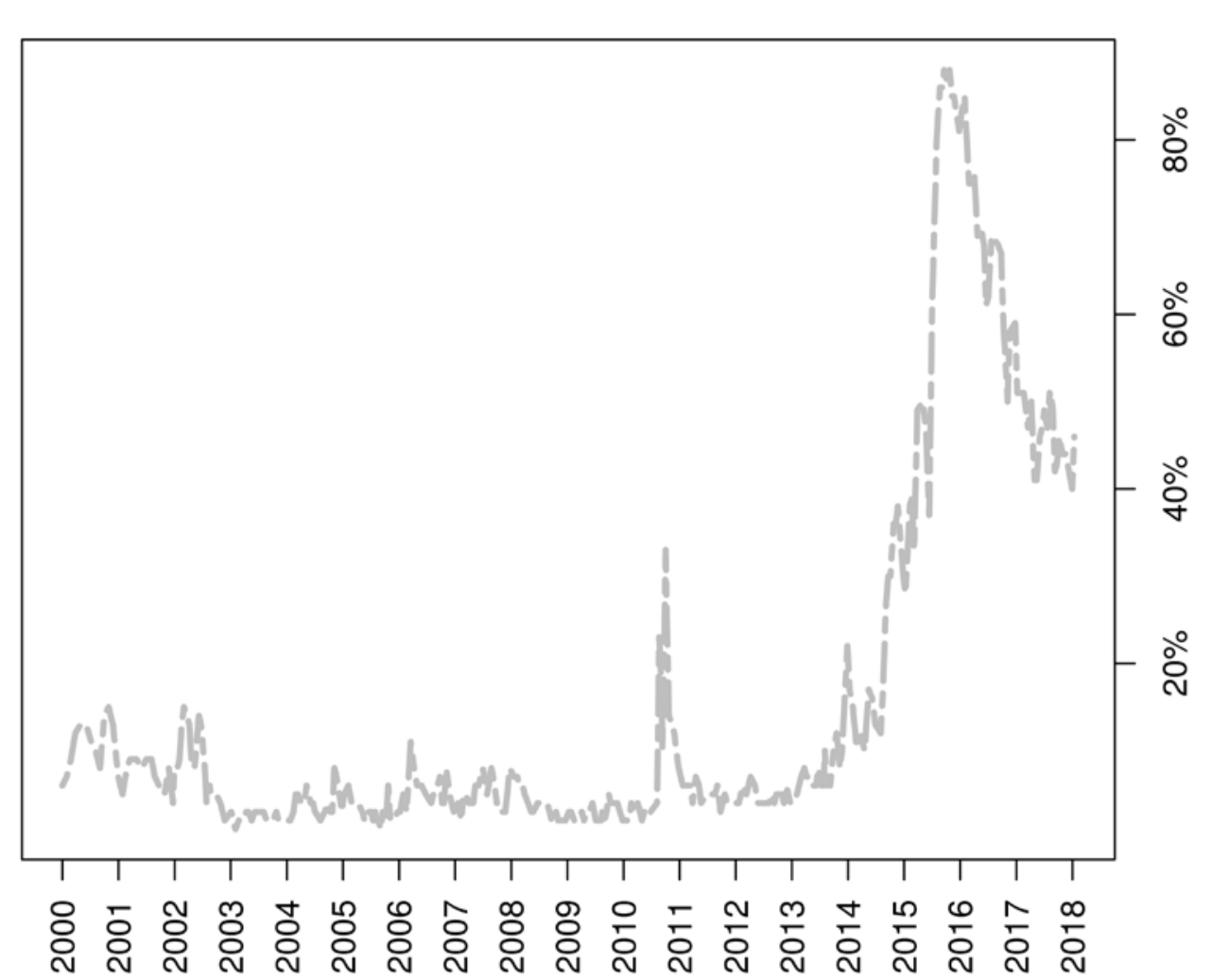

Note: data taken from monthly waves of the Politbarometer survey (Forschungsgruppe Wahlen, 2018) 
Figure 2. Citizens' and speech attention to immigration in the 18th Bundestag term

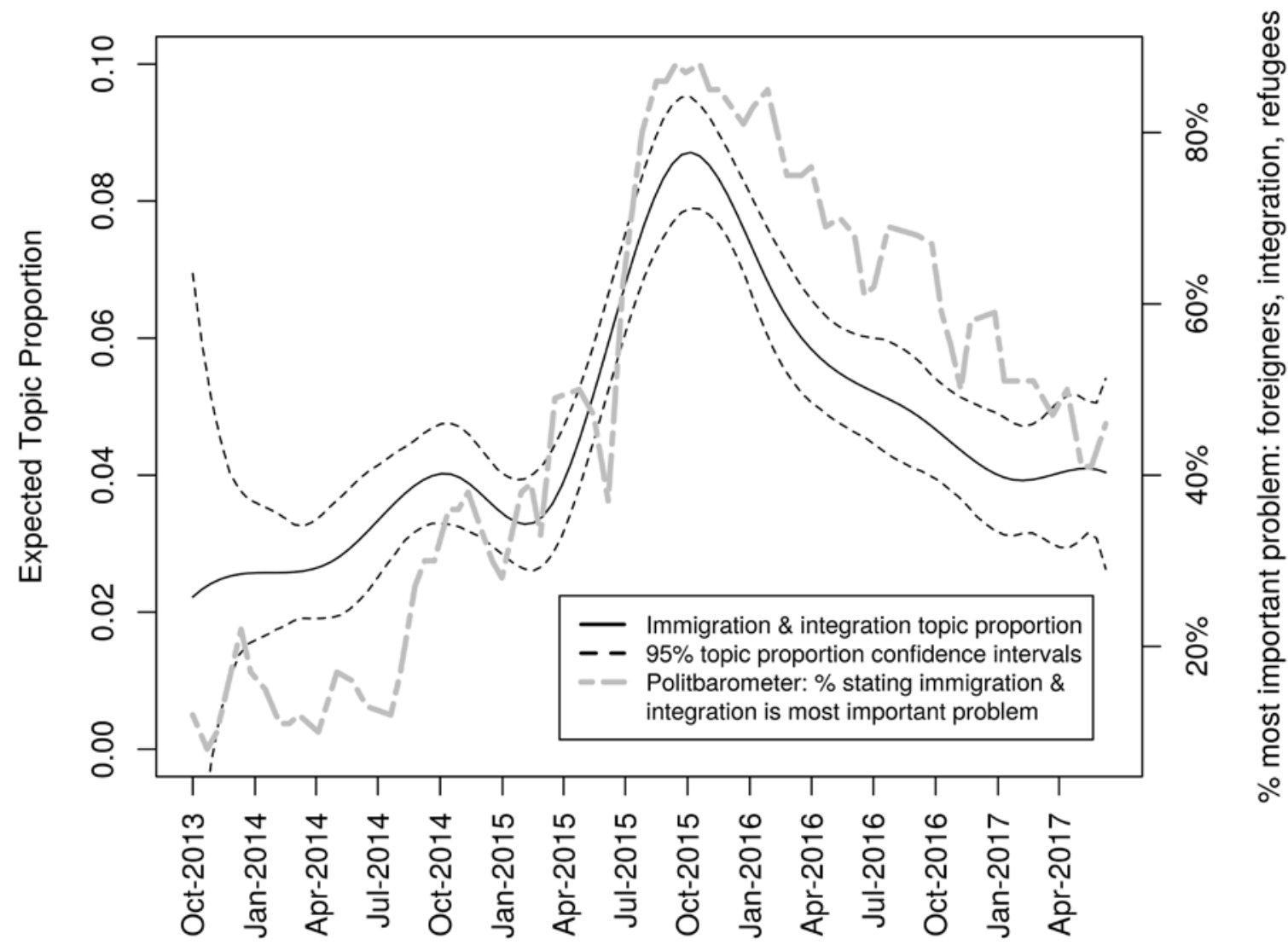

Note: survey data taken from monthly waves of the Politbarometer survey (Forschungsgruppe Wahlen, 2018) 
Figure 3. Predicted speech attention to immigration by visible immigrant-origin legislators, migration-related committee and PPGs with 95\% confidence intervalls
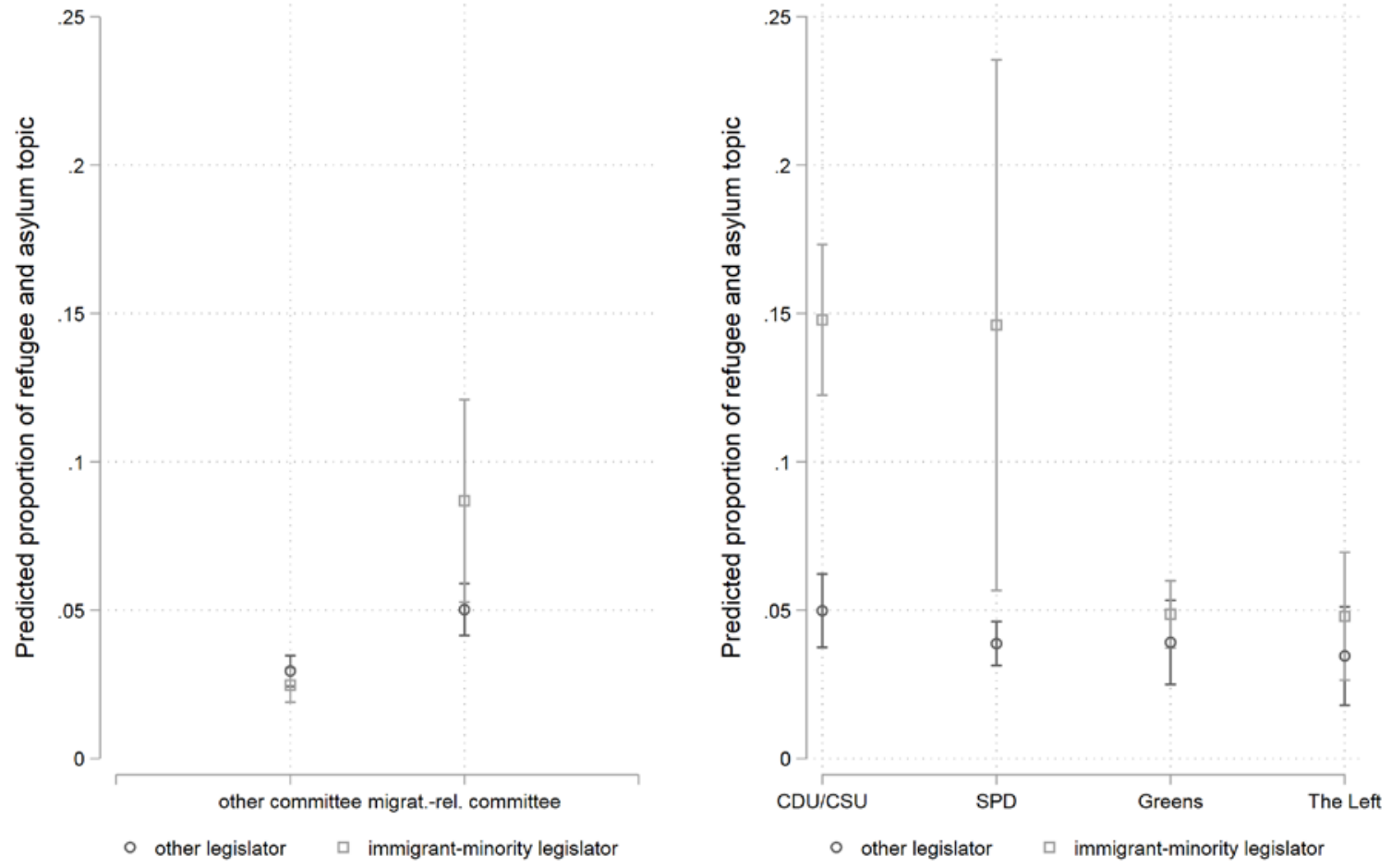

Note: the left-hand panel is based on Model 2 and the right-hand panel is based on Model 3 in Table 2. 
Figure 4. Predicted speech attention to immigration by election mode, government status and vote margin with 95\% confidence intervals
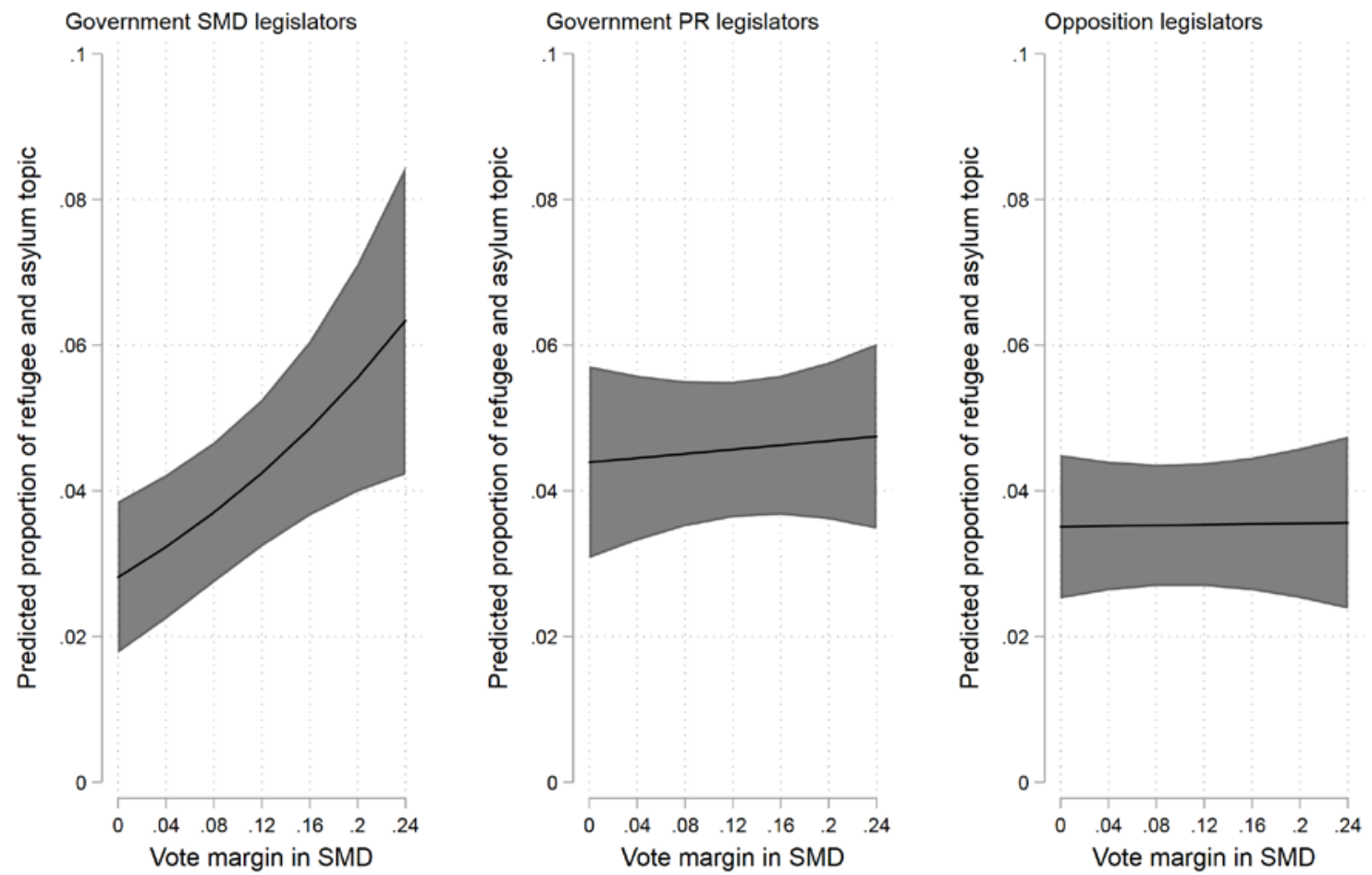

Note: based on Model 5 in Table 2. 
Appendix file to paper

„Immigration-related speechmaking in a party-constrained parliament: evidence from the 'refugee crisis' of the 18th German Bundestag (20132017) 


\section{A1. Pre-processing of the corpus, technical details on the STM and further validation}

\section{analyses}

The STM algorithm represents each document in the corpus as a mixture of a pre-defined number of $\mathrm{k}$ topics, such that each document in the corpus is assigned a vector of $\mathrm{k}$ topic proportions adding up to a value of one (Roberts, Stewart, and Airoldi 2016; Roberts et al. 2014). Therefore, topic proportions are confined to values between zero and one. For example, a k=3 STM may estimate a speech to consist of $20 \%$ of a topic labelled 'EU relations', 30\% 'development aid' and 50\% 'immigration'.

In order to fit an STM to a corpus, analysts must do a number of pre-processing steps. Using the R package 'quanteda' (Benoit et al., 2017) several pre-processing steps were conducted. First, all speeches by the President of the German Bundestag, ministers, junior ministers and chancellor Merkel were discarded. Second, oral contributions containing less than 300 words were excluded as these were mainly interruptions and interposed questions from the plenary audience rather than actual speeches. Third, in order to reduce the dimensionality of word usage, the words were reduced to their stems, capitalisation, numbers and punctuation were removed as well as very frequent and rare stems which appear in more than $20 \%$ or in less than $1 \%$ of all speeches, (Grimmer \& Stewart, 2013). After these steps, 10,379 speeches remained in the dataset, which were then transformed into a document-term matrix representing speeches in rows as frequencies of word stems in columns. Consequently, word order is disregarded as an important feature of the texts which are now represented as 'bags of words'. As such, the corpus is prepared for the STM analysis, which was then conducted using the R package 'stm' (M. Roberts et al., n.d.).

On a technical note, compared to other topic models, the STM estimation provides researchers the opportunity to base the probabilistic estimation on document-specific prior distributions rather than on globally shared priors (M.E. Roberts et al., 2016, pp. 989-990). Thereby, researchers are enabled to base the estimation on document-level covariates they deem 
important for the prevalence of topic proportions (M.E. Roberts et al., 2014). Since it is reasonable to assume that speech attention to the immigration issue in the 18th Bundestag varied as a function of time, the date of a speech is included in the STM estimation as a spline term to model non-linear dynamics over time.

Moreover, analysts need to specify the number of topics before the estimation of the STM. Although there is no clear rule how to choose the number of topics (Roberts et al. 2014), the social science goal of utilising topic models is the revelation of substantively interesting information with regard to the research question at hand (Grimmer and Stewart 2013, 20). As such, an STM with 13 topics was found to be most useful in order to reveal speech attention to the refugee and asylum issue. Regardless of how fine-grained the topic model was parametrised, it was not possible to distinguish different sup-topics to distinguish between refugee and asylum issues and, for example, issues concerning long-term residents and citizens of immigrant-origin, or the immigration of skilled labour.

Nevertheless, validity checks reveal that the found topic is clearly dominated by the refugee and asylum issue. In addition to the analysis of the topic's 'predictive validity' in the main body of the article, the following word cloud, which visualises words most likely to appear in the topic, provides further corroboration that the topic captures mainly words that are strongly related to the issue of refugees and asylum. Furthermore, inspections of speeches with particularly high topic proportions (the full texts of two of those are presented on the following pages) suggest that the found topic is clearly dominated by the refugee and asylum issue. 


\section{Word cloud:}

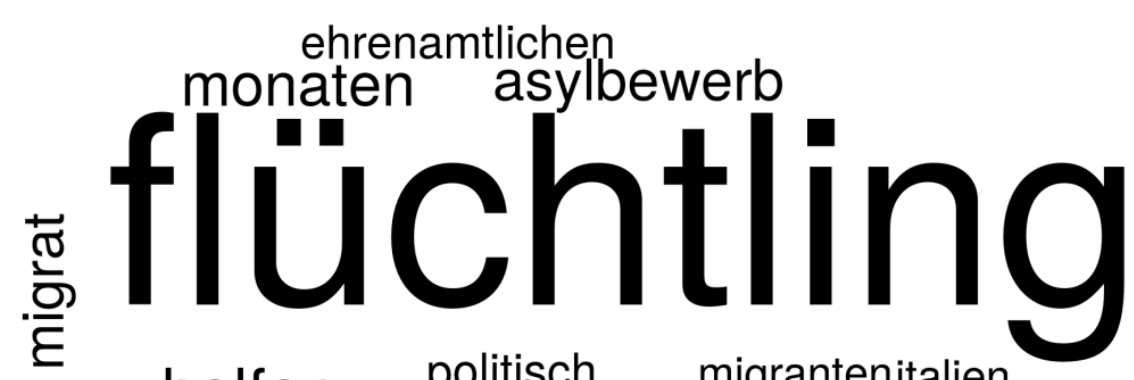

suchen helfen politisch migrantenitalien
staat bundespolizeisprach aufnahm
syrien vergangenen diejenigen braucht aungäng O్neimat not dankbar sicherheit polizei ${ }_{\text {geflüch }}$

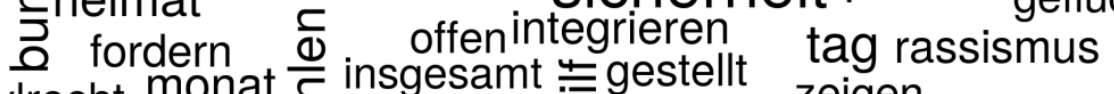
asylrecht monat $\frac{0}{\frac{c}{c}}$ insgesamt signal Nָderzeitklare Ort personen kommunen bewältigen un dingen de Schutz sicheren bayern angesicht erhalten wochen mittelm fluchtschneller lage getan aufgenommen wochen mittelm fluchtschn
bundesamt rund flüchllingspolitik gestern engagieren europäisch solidarität zahl Integr verehrtensicherheitsbehörden asyl stellt
zuwanderung

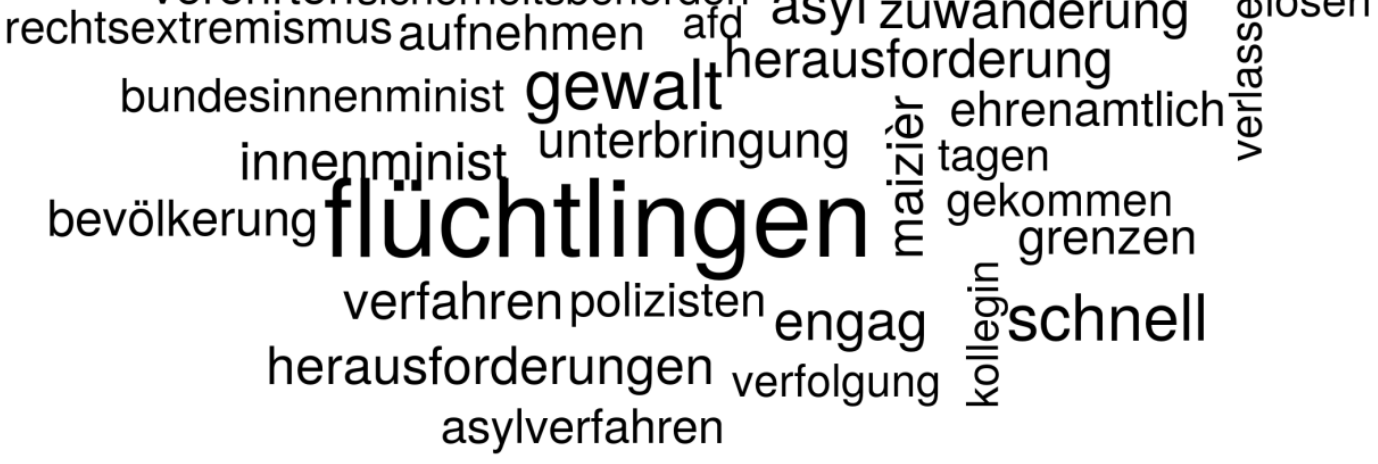

Speech example 1 (Gabriella Heinrich, SPD, $25^{\text {th }}$ September 2014):

“Sehr geehrter Herr Präsident! Meine Damen und Herren! Liebe Kollegen und Kolleginnen! Rund 9000 Flüchtlinge haben in den ersten Monaten dieses Jahres die Grenze überquert. Das Aufnahmeland muss diese Flüchtlinge versorgen, medizinisch betreuen und menschenwürdig unterbringen. Das ist unzweifelhaft eine große Herausforderung für das Aufnahmeland. Die Herausforderung ist so groß, dass gedroht wurde, die Grenze zu kontrollieren. Gemeint war die bayerisch-österreichische Grenze, um die Einreise von weiteren Flüchtlingen zu 
verhindern. Es war der bayerische Ministerpräsident, der die Rückkehr zu Grenzkontrollen forderte und angesichts der Flüchtlingszahlen eine Überforderung Bayerns und Deutschlands beklagte. Meine Damen und Herren, es liegt mir völlig fern, mich mit diesem Vergleich lustig zu machen. Mir ist sehr wohl bewusst, welche Schwierigkeiten unsere Kommunen angesichts der steigenden Flüchtlingszahlen bewältigen müssen. Ich gebe aber zu, dass ich genau daran denken musste, als angesichts der Zahlen, die wir aus Syrien gehört haben, vor ein paar Tagen die Empörung über die zeitweilige Schließung der Grenzen in der Türkei hochkochte. Meine Damen und Herren, Sie kennen die Zahlen. Die Kollegin Roth hat sie heute schon benannt. 130000 Menschen sind innerhalb weniger Tage aus Syrien in die Türkei geflohen. 1,5 Millionen syrische Flüchtlinge befinden sich bereits in der Türkei. 3 Millionen Syrer sind in die Nachbarländer geflohen, neben der Türkei in den Libanon, nach Jordanien, nach Ägypten und in den Irak. Nur 4 Prozent dieser syrischen Flüchtlinge haben in Europa Asyl beantragt. Besonders schwierig ist die Situation der syrischen Frauen. Jede vierte syrische Flüchtlingsfamilie wird von einer Frau geführt. Nur wenige werden von Verwandten unterstützt. Sie sind meist völlig auf sich selbst gestellt, müssen ihre Familien irgendwie durchbringen und haben häufig keine Chance auf eine existenzsichernde Arbeit. Ohne einen begleitenden Ehemann werden sie gedemütigt und belästigt. Sexuelle Übergriffe sind an der Tagesordnung. Das Flüchtlingshilfswerk der Vereinten Nationen hat deswegen zur verstärkten Unterstützung dieser Frauen aufgerufen. Meine Damen und Herren, die Bundesregierung macht mit der geplanten Flüchtlingskonferenz zur Hilfe der Anrainerstaaten deutlich, dass das Thema Flüchtlinge ganz oben auf der politischen Agenda steht. Die Probleme der Frauen müssen Teil dieser Konferenz werden. Es wurde bereits erwähnt, dass Deutschland aus humanitären Gründen 20000 syrische Flüchtlinge aufgenommen hat. Das ist ein ganz wichtiger Beitrag. Es ist auch richtig, wenn wir mehr Solidarität innerhalb der Europäischen Union einfordern. Europa ist mehr als nur eine Wirtschaftsgemeinschaft. Wir müssen uns zuallererst als Wertegemeinschaft begreifen, wenn wir eine gemeinsame Zukunft 
haben wollen. Dazu gehört das Recht auf Asyl in den Ländern der Europäischen Union. Aber welche Handlungen leiten wir von diesem Wert ab? Europa kann nicht von den Nachbarländern Syriens erwarten, dass sie 3 Millionen Flüchtlinge angemessen versorgen, jedoch die Aufnahme von Flüchtlingen weitgehend ablehnen. Wir können es auch dann nicht erwarten, wenn wir viel Geld für die humanitäre Hilfe zur Verfügung stellen. Nehmen wir uns ein Beispiel an den Kommunen in Deutschland. Vielerorts bilden sich Unterstützerkreise, in denen sich Nachbarn und Kirchengemeinden um die Flüchtlinge kümmern, Ärzte und Krankenschwestern helfen, die Ankommenden medizinisch zu versorgen, wie in meiner Heimatstadt Nürnberg. Es gibt durchaus viele, die verstanden haben, dass über 50 Millionen Flüchtlinge auf der Welt auch unseren Teil an Solidarität einfordern, und sie erwarten dies auch von unserer Politik. Die Wertegemeinschaft steht vor einer ungeheuren Herausforderung. Terrorismusbekämpfung und Massenmorde zu stoppen, ist die eine Seite, schnelle humanitäre Hilfe ist die andere. Seit 2012 hat Deutschland die Krisenregion mit über 600 Millionen Euro unterstützt. Angesichts der aktuellen Flüchtlingsströme - nicht nur in die Türkei - ist der Bedarf an dieser Hilfe aber nicht weniger, sondern mehr geworden. Deswegen setzt sich die SPD in den Haushaltsberatungen für eine Aufstockung der humanitären Hilfe ein. Wir haben heute gehört, dass dies in der Koalition Konsens ist. Es muss uns gelingen, das Morden und Zerstören in Syrien und im Irak zu beenden. Die Weltgemeinschaft wird sich diesmal aber auch beim Wiederaufbau langfristig engagieren müssen, um die Weichen für einen nachhaltigen Frieden zu stellen. Erinnern wir uns an die Solidarität, die wir als Deutsche nach dem Zweiten Weltkrieg erfahren haben. Danke schön.“

\section{Speech example 2 (Ulla Jelpke, Die Linke, 20 ${ }^{\text {th }}$ March 2014):}

"Frau Präsidentin! Meine Damen und Herren! Der grausame Krieg in Syrien läuft bereits seit drei Jahren, und weit mehr als 100000 Menschen sind ums Leben gekommen. 10 Millionen Menschen sind auf der Flucht, davon ungefähr 7,5 Millionen im Land selber auf der Suche 
nach Alternativen. 2,5 Millionen registrierte Flüchtlinge befinden sich in Anrainerstaaten Syriens und in Ägypten. Ich muss wirklich sagen: Es ist eine Schande, dass es bis heute nur einem Bruchteil der Flüchtlinge gelungen ist, in die Europäische Union zu kommen, um Zuflucht zu finden. Meine Damen und Herren, an den Außengrenzen der EU treffen Flüchtlinge auf eine immer massivere und brutalere Abschottung. Die Landesgrenzen Griechenlands und Bulgariens zur Türkei wurden zum Beispiel mit Zäunen und Stacheldraht abgeriegelt. Soldaten greifen Flüchtlinge in der Ägäis auf und schaffen sie zurück in die Türkei. Wie viele Menschen die gefährliche Überfahrt über die Ägäis und das Mittelmeer nicht überleben, weiß wirklich niemand. Täglich sterben an den Außengrenzen der EU - das ist leider bittere Wahrheit - unerträglich viele Menschen. Das muss dringend beendet werden. Trotz all dieser Abschottungsbemühungen haben es in den vergangenen zwei Jahren etwa 70 000 Flüchtlinge aus Syrien geschafft, in die Europäische Union zu fliehen. Gut ein Drittel wurden in der Tat in der Bundesrepublik aufgenommen. Im gleichen Zeitraum wurde gerade einmal 12000 Menschen die Zusage für eine Aufnahme in einem EU-Staat gegeben; nur diese haben also sichere und legale Einreisemöglichkeiten. Von ihnen nimmt allein die Bundesrepublik 10000 Flüchtlinge auf. Doch das bedeutet noch lange nicht, dass sich die Bundesrepublik auf ihren Verdiensten ausruhen darf. Ein Vergleich, um sich das einfach einmal vorzustellen: Im Libanon hat es einen Bevölkerungszuwachs um 19 Prozent gegeben. Das würde für Deutschland bedeuten, dass es innerhalb von zwei Jahren einen Bevölkerungszuwachs von 15 Millionen Menschen gegeben hätte. Die Bundesregierung bzw. die Bundesrepublik kann mehr tun, muss mehr tun, und es muss vor allen Dingen syrischen Flüchtlingen schneller und unbürokratischer geholfen werden. Syrische Asylsuchende, die über einen anderen EU-Staat nach Deutschland eingereist sind, geraten wirklich in die Mühlen der Asylbürokratie. Man muss sich einfach einmal vorstellen: Sie fliehen aus ihrem Land vor Krieg, sie fliehen über die Meere und gefährden ihr Leben, dann kommen sie nach Deutschland und werden mit der Dublin-Verordnung konfrontiert. Und was passiert? Als 
Allererstes gehen sie in Abschiebegefängnisse, weil sie überstellt werden sollen. Das ist ein Verfahren, das unbedingt abgeschafft werden muss. Deswegen fordert die Linke auch, die Dublin-Verordnung ganz schnell für syrische Flüchtlinge auszusetzen. Es ist wirklich ein bürokratischer Irrsinn, der hier betrieben wird. Man muss wirklich an das Bundesinnenministerium sowie die Innenminister der Länder und der europäischen Staaten appellieren, dass das geändert wird. Es ist hier schon angesprochen worden, dass viele Flüchtlinge Verwandte in Deutschland haben. Auch diesen muss unbegrenzt und unbürokratisch ermöglicht werden, dass sie von den entsprechenden Ländern aufgenommen werden und zu ihren Familien reisen können. Übrigens wäre das die schnellste und einfachste Art, den Flüchtlingen zu ermöglichen, ein Leben in Sicherheit zu führen. Daneben muss sich die Bundesrepublik auch weiterhin an humanitären Aufnahmeprogrammen des UNHCR beteiligen. Meine Damen und Herren, auch auf EU-Ebene muss mehr getan werden. Deutschland muss Druck machen. Ohne mit der Wimper zu zucken, werden Gelder in Milliardenhöhe bereitgestellt, um die Grenzen abzusichern. Wir meinen, dass die Gelder viel sinnvoller für Flüchtlinge eingesetzt wären. Das Wichtigste ist: Wir sollten nicht noch mehr Öl ins Feuer gießen und endlich dafür sorgen, dass Waffenlieferungen nach Syrien, und zwar an alle Seiten, gestoppt werden. Ich danke Ihnen." 


\section{A2. Descriptive statistics}

\begin{tabular}{|c|c|c|c|c|c|}
\hline Variable & $\mathbf{N}$ & Mean & Std. Dev. & Min & Max \\
\hline $\begin{array}{l}\text { Speech attention to } \\
\text { immigration }\end{array}$ & 10,379 & 0.04 & 0.10 & 0.00 & 20.80 \\
\hline Party: CDU/CSU & 10,379 & 0.35 & 0.48 & 0 & 1 \\
\hline Party: SPD & 10,379 & 0.27 & 0.45 & 0 & 1 \\
\hline Party: Greens & 10,379 & 0.19 & 0.39 & 0 & 1 \\
\hline Party: The Left & 10,379 & 0.18 & 0.39 & 0 & 1 \\
\hline $\begin{array}{l}\text { Immigrant-minority } \\
\text { legislator }\end{array}$ & 10,379 & 0.05 & 0.23 & 0 & 1 \\
\hline Election mode: SMD & 10,379 & 0.37 & 0.48 & 0 & 1 \\
\hline $\begin{array}{l}\text { Vote margin in } \\
\text { constituency }\end{array}$ & 9,900 & 0.13 & 0.11 & 0.00 & 0.53 \\
\hline Days till election & 10,379 & 720.47 & 381.75 & 86 & 1433 \\
\hline $\begin{array}{l}\text { Legislative role: } \\
\text { Megaseat }\end{array}$ & 10,379 & 0.24 & 0.43 & 0 & 1 \\
\hline $\begin{array}{l}\text { Committee: migration- } \\
\text { related }\end{array}$ & 10,379 & 0.61 & 0.49 & 0 & 1 \\
\hline Seniority & 10,379 & 5.57 & 6.08 & 0 & 37 \\
\hline Gender: female & 10,379 & 0.38 & 0.49 & 0 & 1 \\
\hline East & 10,379 & 0.16 & 0.37 & 0 & 1 \\
\hline $\begin{array}{l}\text { Foreigner share in } \\
\text { constituency }\end{array}$ & 9,900 & 0.10 & 0.06 & 0.01 & 0.28 \\
\hline $\begin{array}{l}\text { Population share older } \\
\text { than } 60 \text { in constituency }\end{array}$ & 9,900 & 0.26 & 0.03 & 0.20 & 0.34 \\
\hline $\begin{array}{l}\text { Unemployment rate in } \\
\text { constituency }\end{array}$ & 9,900 & 0.07 & 0.03 & 0.02 & 0.15 \\
\hline
\end{tabular}




\section{Appendix references}

Benoit, K., Watanabe, K., Nulty, P., Obeng, A., Wang, H., Lauderdale, B. \& Lowe, W.L. (2017). 'quanteda: Quantitative Analysis of Textual Data'. R package version: 0.9.9.67. London.

Roberts, M., Stewart, B. \& Tingley, D. (n.d.). stm: R Package for Structural Topic Models. Journal of Statistical Software. Retrieved from https://github.com/bstewart/stm/blob/master/inst/doc/stmVignette.pdf?raw=true

Roberts, M.E., Stewart, B.M. \& Airoldi, E.M. (2016). A Model of Text for Experimentation in the Social Sciences. Journal of the American Statistical Association 111(515): 9881003.

Roberts, M.E., Stewart, B.M., Tingley, D., Lucas, C., Leder-Luis, J., Gadarian, S.K., ... Rand, D.G. (2014). Structural topic models for open-ended survey responses. American Journal of Political Science 58(4): 1064-1082. 